\title{
L'Approche de l'Analyse Situationnelle pour l'évaluation des services de planification familiale et de santé de la reproduction: Manuel de recherche
}

\author{
Robert A. Miller \\ Population Council \\ Andrew A. Fisher \\ Population Council \\ Kate Miller \\ Population Council \\ Lewis Ndhlovu \\ Population Council \\ Baker Ndugga Maggwa \\ Population Council \\ Follow this and additional works at: https://knowledgecommons.popcouncil.org/departments_sbsr-rh \\ fre next page for additional authors \\ Part of the Demography, Population, and Ecology Commons, Family, Life Course, and Society \\ Commons, International Public Health Commons, Medicine and Health Commons, and the Women's \\ Health Commons
}

How does access to this work benefit you? Let us know!

\section{Recommended Citation}

Miller, Robert A., Andrew A. Fisher, Kate Miller, Lewis Ndhlovu, Baker Ndugga Maggwa, lan Askew, Diouratie Sanogo, and Placide Tapsoba. 1999. "L'Approche de l'Analyse Situationnelle pour l'évaluation des services de planification familiale et de santé de la reproduction: Manuel de recherche." New York:

Population Council. 


\section{Authors}

Robert A. Miller, Andrew A. Fisher, Kate Miller, Lewis Ndhlovu, Baker Ndugga Maggwa, lan Askew, Diouratie Sanogo, and Placide Tapsoba 


\section{LRPproche de PRallyse Siturtionnelle}

pour P'valuation des services de planification fomiliale et dle sante de la reproduction

Manuel de recherche

Robert Miller - Andrew Fisher - Kate Miller Lewis Ndhlovu • Baker Ndugga Maggwa • Ian Askew Diouratié Sanogo Placide Tapsoba 



\section{LApproche de l'Analyse Situationnelle pour l'évaluation des services de planification familiale et de santé de la reproduction}





\section{L'Approche de l'Analyse Situationnelle pour l'évaluation des services de planification familiale et de santé de la reproduction}

\section{Manuel de recherche}

Robert Miller • Andrew Fisher • Kate Miller

Lewis Ndhlovu • Baker Ndugga Maggwa - Ian Askew Diouratié Sanogo • Placide Tapsoba

\footnotetext{
(2) Population Council 


\section{Library of Congress Cataloging-in-Publication Data}

Situation analysis approach to assessing family planning and reproductive health services. French

L'approche de l'analyse situationnelle pour l'évaluation des services de planification familiale et de santé de la reproduction : manuel de recherche / Robert Miller... [et al.].

p. $\mathrm{cm}$.

"Projet de recherche opérationnelle et d'assistance technique en Afrique." Includes bibliographical references (p. ).

ISBN 0-87834-097-1 (pbk. : alk. paper)

1. Birth control-Research. 2. Birth control-Data processing. 3. Maternal health services-Evaluation. 4. Evaluation research (Social action programs) I. Miller, Robert, 1942II. Population Council. [HQ763.5.S5714 1999] $363.9^{\prime} 6^{\prime} 07-\mathrm{dc} 21$

99-20485

CIP

Le Population Council cherche à améliorer le bien-être et la santé de la reproduction des générations actuelles et futures à travers le monde et à aider à l'établissement d'un équilibre humain équitable et durable entre les populations et les ressources. Le Population Council effectue des recherches dans les domaines de la biomédecine, des sciences sociales et de la santé publique et aide à renforcer les outils de recherche dans les pays en voie de développement. Le Population Council est une organisation non gouvernementale à but non lucratif, créée en 1952 ; il est dirigé par un Conseil d'Administration dont les membres sont originaires de différents pays. Son siège à New York apporte un soutien à tout un réseau de bureaux nationaux et régionaux.

Population Council

One Dag Hammarskjold Plaza

New York, New York 10017 USA

tel : (212) 339-0500

fax : (212) 755- 6052

email : pubinfo@popcouncil.org

http://www.popcouncil.org

Les auteurs sont des employés du Population Council. Robert Miller, Associé de Programme, New York ; Andrew Fisher, Directeur de Programme de Planification Familiale et Associé de Programme, New York ; Kate Miller, précédemment Associé de Programme, New York, actuellement étudiante doctorale, Département de Démographie, University of Pennsylvania, Philadelphia; Lewis Ndhlovu, Associé de Programme, Nairobi ; Baker Ndugga Maggwa, Associé de Programme, Nairobi ; Ian Askew, Directeur de Projet et Associé de Programme, Nairobi ; Diouratié Sanogo, Directeur Adjoint de Projet et Associé de Programme, Dakar ; Placide Tapsoba, Associé de Programme, Dakar.

Cette publication a été réalisée par le Projet Recherches Opérationnelles et Assistance Technique II (RO/AT II) en Afrique, projet $N^{\circ}$ 963-3030 et le Programme Expansion des Choix Contraceptifs (ECC) du Population Council. Le Projet RO/AT II est financé par la Division Population de l'Agence Américaine pour le Développement International (USAID) sous le contrat $\mathrm{N}^{\circ} \mathrm{CCP}-3030-\mathrm{C00}-00-3008-00$, stratégies pour améliorer les prestations de services en matière de Planification Familiale. Le Programme Expansion des Choix Contraceptifs est aussi financé par l'USAID par l'accord de coopération N CCP-3050-A-00-4013.

Il est permis de reproduire ce document en tout ou en partie sans la permission des auteurs à condition de ne pas en retirer de profits d'ordre financier. Les lecteurs sont invités à faire des commentaires ou des suggestions. Des rapports de la part de ceux qui effectuent des études d'analyse situationnelle seraient fort appréciés.

(ㄱ1999 par The Population Council, Inc. Tous droits réservés. 


\section{Contents}

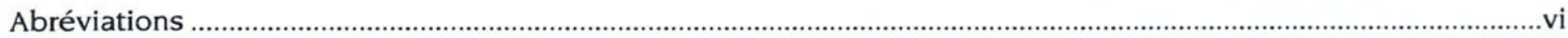

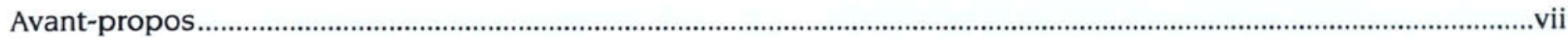

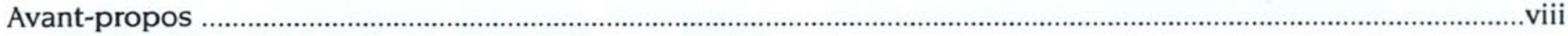

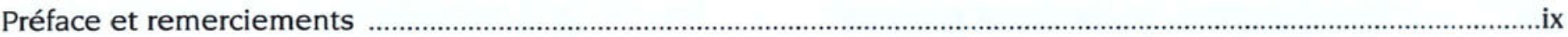

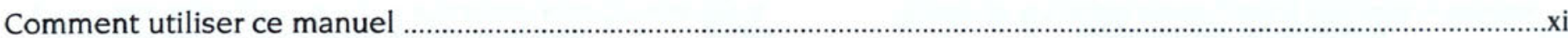

\section{Chapitre 1}

La méthodologie de l'étude d'Analyse Situationnelle ..1

Objectifs d'une étude d'Analyse Situationnelle

Eléments de base

Procédé de recherche

Instruments de collecte des données

Exécution de l'étude

Utilisation des études d'Analyse Situationnelle

Etudes d'impact et Analyse Situationnelle

Recherche opérationnelle et Analyse Situationnelle.......11

La diffusion des études d'Analyse Situationnelle

Problèmes d'ordre méthodologique

Conclusions

Références

\section{Chapitre 2}

Exécution de l'étude .16

Problèmes d'ordre éthique dans

l'exécution des études d'AS

Etablissement des objectifs et révision des

questionnaires .18

Programme de formation de l'équipe de recherche ....19

Rôle du Chef d'équipe/Superviseur

L'équipe de recherche et une journée typique de travail .21

Astuces pour réaliser une bonne interview .22

Astuces pour réaliser une bonne observation .23

Après la collecte des données

\section{Chapitre 3}

Instruments et guides question par question .25

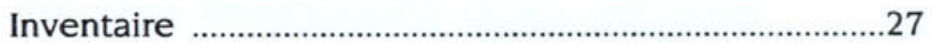

Inventaire : guide question par question 41

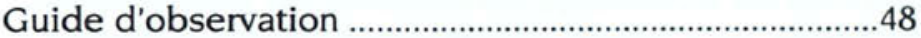

Observation : guide question par question

.57

Interview de sortie de la cliente de

planification familiale

Interview de sortie de la cliente de planification familiale :

guide question par question

Interview du personnel

Interview du personnel :

guide question par question ..........................................126

Interview de sortie de la cliente SMI

Interview de sortie de la cliente SMI :

guide question par question

\section{Chapitre 4}

Analyse des données et rédaction des rapports ......161

Saisie et nettoyage des données ...................................161

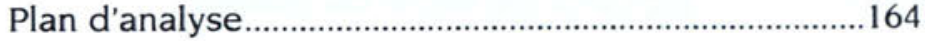

Notes sur le plan d'analyse ............................................185

Listes des échantillons d'indicateurs .............................188

Echantillons de graphiques 


\section{Abréviations}

\begin{tabular}{|c|c|c|c|}
\hline AIBEF & Association Ivoirienne pour le & LT & Ligature des trompes \\
\hline & Bien-Etre Familial & MAMA & Méthode de l'allaitement maternelle \\
\hline APF & Association de planification familiale & & et de l'aménorrhée \\
\hline $\mathrm{AQ}$ & Assurance de qualité & MDS & Module de disponibilité des services \\
\hline AS & Analyse situationnelle & MS & Ministère de la santé \\
\hline AT & Assistance téchnique & MST & Maladie sexuellement transmissible \\
\hline AV & Audio-visuel & ONG & Organisation non gouvernementale \\
\hline BCG & Bacille Calmette-Guérin (vaccin) & PEV & Programme étendu de vaccinations \\
\hline BKKBN & Conseil national coordinateur de la & PF & Planification familiale \\
\hline & & PFN & Planification familiale naturelle \\
\hline CAP & Connaissance, attitude, et pratique & POP & Pilule progestative \\
\hline CIOMS & $\begin{array}{l}\text { Council for the International } \\
\text { Organization of the Medical Sciences }\end{array}$ & PPS & Point de prestation de services \\
\hline $\mathrm{COC}$ & Pilule combinée & PRICOR & $\begin{array}{l}\text { Recherche opérationnelle sur les } \\
\text { soins de santé primaires }\end{array}$ \\
\hline DBC & Distribution à base communautaire & RO & Recherche opérationnelle \\
\hline DDR & Date des dernières règles & SIDA & Syndrome d'immuno-déficience \\
\hline DIU & Dispositif intra-utérin & & acquis \\
\hline DPT & Diphtérie, pertussis, et tétanos & SIG & Système d'information de gestion \\
\hline & & SMI & Santé maternelle et infantile \\
\hline EBF & Educateur du bien-être de la famille & SR & Santé de la reproduction \\
\hline EDS & Enquête démographique et de santé & TA & Tension artérielle \\
\hline EMF & Enquête mondiale de fécondité & USAID & Agence américaine pour le \\
\hline ESD & Equipes de santé du district & & développement international \\
\hline IAG & Infection des voies génitales & VIH & Virus d'immuno-déficience humain \\
\hline IEC & $\begin{array}{l}\text { Information, éducation, et } \\
\text { communication }\end{array}$ & ZNFPC & $\begin{array}{l}\text { Conseil national de la planification } \\
\text { familiale de Zimbabwe }\end{array}$ \\
\hline
\end{tabular}

IP

Inflammation pelvienne 


\section{Avant-propos}

La mission qui incombe aux responsables des programmes de planification familiale, celle des professionnels de la planification familiale, est d'assurer aux clientes des services ponctuels, sûrs et respectueux. Les responsables de programmes qui prennent cette mission au sérieux ont la responsabilité d'orchestrer les ressources, le personnel et les structures de services pour répondre aux besoins de plusieurs milliers et, dans certains cas, plusieurs millions d'individus. Cependant, dans le passé, il y a eu peu d'outils pour guider les responsables préoccupés par la qualité des soins que les clientes reçoivent. Bien qu'au cours des dernières années il y ait eu une bonne information au niveau national sur la qualité des ressources telle que l'approvisionnement ininterrompu en contraceptifs, et les résultats au niveau de la société tels que la prévalence contraceptive, d'une manière générale, les responsables de programmes n'ont pas été informés de ce que les agents de planification familiale font en réalité, le degré auquel ils sont préparés à répondre aux besoins réels des client(e)s, et ce qui est encore plus important, ils ne se sont pas fait une idée de ce que les client(e)s reçoivent en termes de soins.

C'est là qu'intervient l'outil appelé Analyse Situationnelle. Il fournit un lien plus que nécessaire entre le responsable de programme et la cliente qu'il essaie de servir. Les données recueillies par l'Analyse Situationnelle offrent une image représentative du fonctionnement des composantes du système de prestation de services et permettent de "voir" l'expérience de la cliente. L'Analyse Situationnelle aide le responsable de programme à atteindre le plus grand but en termes de gestion-celui d'une administration efficace d'un service de soins de santé vital-tout en gardant fermement le regard fixé sur l'ultime butfournir des soins de qualité aux individus qui les sollicitent.
Judith Bruce

Directeur du Programme Genre, Famille et Développement Population Council 


\section{Avant-propos}

La prestation de services de planification familiale de qualité met à la fois l'accent sur l'accès et la qualité. Elle est capitale à la réalisation des deux objectifs interdépendants que sont la satisfaction des besoins des individus et la réalisation du succès programmatique. En tant qu'utilisateur des données et de la littérature scientifique sur la planification familiale, j'ai trouvé que l'Analyse Situationnelle était une mine d'or d'informations utiles. C'est le premier outil de collecte systématique de données qui documente de manière crédible ce qui se passe à l'intérieur de la formidable "boîte noire" de prestation de services. A mon avis, elle se classe avec les Enquêtes Démographiques et de Santé comme une méthode riche et originale pour notre domaine d'activité.

Dans le même temps, il importe de garder à l'esprit certaines des limites de l'Analyse Situationnelle. Elle représente une photo instantanée de la réalité de la prestation des services, et les résultats sont indubitablement influencés par la simple présence des observateurs. De même, l'importante information que l'Analyse Situationnelle fournit a tendance à engendrer un coût élevé, et les résultats sont mieux interprétés à la lumière du contexte de prestation de services à partir duquel ils sont obtenus. Nous devons continuer à améliorer la méthodologie de l'Analyse Situationnelle, à développer et améliorer d'autres outils pour évaluer et renforcer l'accès et la qualité de la prestation de services.
L'un des attributs les plus importants de l'Analyse Situationnelle est la simplicité de ses résultats. Par exemple, prenons une Analyse Situationnelle qui montre que la moitié de tous les sites de prestation de services ont eu une rupture des injectables au cours des six derniers mois, ou que l'on n'a discuté d'effets secondaires avec les nouvelles clientes que dans la moitié des cas. De tels résultats sont directs pour les responsables de programmes et tous ceux qui sont concernés. Ils sont aussi très courants. En effet, l'une des principales leçons apprises de l'Analyse Situationnelle est le grand fossé entre l'idéal et la réalité. D'une part de tels résultats peuvent être décourageants, d'autre part ils peuvent apporter aussi de l'espoir. Ils représentent des situations qui peuvent (du moins théoriquement) être améliorées facilement. A mon avis, la leçon la plus importante tirée des résultats de l'Analyse Situationnelle est l'énorme amélioration qui peut être apportée en matière d'accès et de qualité potentiellement à notre portée. Des outils bien utilisés tels que l'Analyse Situationnelle peuvent aider à traduire de telles améliorations en réalité. 


\section{Préface et remerciements}

Les études d'Analyse Situationnelle (AS) ont commencé en Afrique. La première a été réalisée au Kenya en 1989 avec la collaboration de la Division de la Santé Familiale du Ministère de la Santé. Depuis cette période, les AS ont été réalisées dans une trentaine de pays à travers le monde avec la collaboration et le soutien de responsables de programmes, ainsi que le dur labeur et l'engagement de centaines de chercheurs, enquêteurs et superviseurs. Nous sommes très reconnaissants envers tous ceux qui ont été impliqués dans la conduite de ces études pour leur enthousiasme, leur dur labeur et leur volonté à endurer les difficultés de la recherche sur le terrain. Nous sommes aussi très reconnaissants envers les centaines d'agents de planification familiale et de santé de la reproduction qui ont réservé un accueil chaleureux à nos équipes de recherche dans leur lieu de travail, qui ont souvent trouvé pour elles des endroits pour dormir, ont ouvert leurs armoires pour inspection, ont permis à ce que leurs patientes soient interviewées et observées, et qui ont répondu à nos nombreuses questions avec patience.

Bien que le développement de cette méthodologie ait reçu un appui important de la part des collègues travaillant dans des projets de recherche dans diverses régions, les instruments originaux sont nés en Afrique et cette itération particulière peut toujours porter une perspective africaine. Par exemple, les pays africains ont développé des programmes intégrés de santé maternelle et infantile et de planification familiale (SMI/PF) qui fonctionnent au sein des ministères de la santé. Beaucoup de pays d'Asie et d'Amérique latine disposent de systèmes de planification familiale à prédominance indépendante. Pendant que le paradigme du Caire encourageait que l'attention soit accordée aux questions plus globales en matière de santé de la reproductionprincipalement le VIH/SIDA et les maladies sexuellement transmissibles-les chercheurs africains étaient particulièrement bien placés et supportés dans leurs efforts à élargir leur couverture sur des questions de santé de la reproduction. Les instruments actuels de l'AS reflètent l'approche plus vaste et intégrée de la santé de la reproduction bien que perçue sous l'angle de la planification familiale. A cause du fait que beaucoup de pays africains ne disposent pas d'un Système d'Information de Gestion (SIG) qui fonctionne, les instruments de l'Analyse Situationnelle recueillent une importante information sur les produits, le niveau des stocks, la fréquence d'utilisation des services, etc., information qui, dans les pays d'Asie et d'Amérique latine, provient souvent d'un SIG en état de fonctionnement.

Bien qu'elle ait été conçue à l'intention des programmes africains, la méthodologie de l'AS a tiré profit de contributions innovatrices des collègues d'autres régions. Par exemple, le premier lien entre les PPS de l'Analyse Situationnelle et les grappes de l'EDS dans lesquelles ils étaient situés a été fait au Pérou sous la direction de James Foreit. D'autres innovations qui découlent de l'expérience de l'AS du Pérou incluent un plan d'étude des circuits d'approvisionnement et le premier module de pharmacie. Huntington et al.' ont réalisé une étude sur la fiabilité de l'observation de l'AS en Turquie et ont utilisé la méthodologie pour se focaliser sur les services de

'D. Huntington, K. Miller, and B. Mensch, 1996. "The reliability of the situation analysis observation guide." Studies in Family Planning 27,5: 277-282. 
stérilisation et les services d'avortement. Il serait difficile de représenter tous les ajustements intéressants qui ont été apportés à la méthodologie et les innovations créatrices qui sont en cours au fur et à mesure que les chercheurs dans d'autres régions adaptent le matériel pour faire ressortir leurs préoccupations nationales et régionales particulières.

Le présent volume représente notre tentative de mise à jour des procédures et matériels selon leur évolution au cours des huit dernières années. Nous ne doutons pas du fait que beaucoup de questions supplémentaires nécessitent davantage de débat, de test et de changement; et que les instruments aussi bien que les procédures pourraient être ajustés aux nouvelles applications et aux situations spécifiques. Les principales questions non résolues sont les suivantes : le nombre de variables incluses dans les études d'AS devrait-il être réduit pour des raisons de maniabilité, ou augmenté pour couvrir un nombre sans cesse croissant de problèmes de recherche? Les études d'AS devraient-elles être intégrées aux études basées sur la population, le cas échéant dans quelles circonstances, pour obtenir un tableau plus complet de l'offre et de la demande ? Comment les composantes santé de la reproduction de l'étude (et naturellement l'ensemble des systèmes de santé) peuvent-elles être évaluées de manière plus adéquate et indépendamment appréciées à travers l'application d'une méthodologie similaire mais plus étendue ? Les instruments doivent-ils être standardisés pour promouvoir les comparaisons au niveau national, ou ajustés de façon continue pour répondre aux préoccupations du pays et aux problèmes de gestion en évolution ? Comment les études d'AS peuvent-elles jouer un rôle dans la recherche de l'impact de la qualité des services sur le comportement des clientes ? Et comment les données collectées peuvent-elles être facilement disponibles pour un grand nombre de chercheurs et de responsables de programmes ?

A travers le processus de développement et de mise en oeuvre de l'AS, nous avons reçu une assistance considérable (financière aussi bien que technique) de l'USAID Washington et de nombreuses Missions de l'USAID. Nous sommes très reconnaissants envers l'USAID pour cette assistance. Qu'elle trouve ici également le témoignage de notre gratitude pour son haut niveau d'encouragement, son utilisation fréquente des résultats de l'étude, son assistance technique et ses nombreuses suggestions qui ont contribué à renforcer la méthodologie. Nous avons tiré aussi énormément profit des commentaires, suggestions, l'ouverture à la discussion, et de la recherche active de nombreux collègues du Population Council—ceux de New York autant que ceux basés dans les autres bureaux régionaux à travers le monde. Leur nombre est si élevé que nous hésitons à fournir une liste de peur d'omettre un collègue méritant. De même, nos collègues dans les agences de coopération de l'USAID ont soutenu entièrement cet effort en aidant à la mise en oeuvre des études sur le terrain, en encourageant la dissémination et l'utilisation des résultats de l'étude, et en enseignant à leurs étudiants et collègues comment utiliser cette méthodologie et l'améliorer.

Bien que le mérite du développement de cette méthodologie revienne à de nombreuses personnes, nous prenons l'entière responsabilité des erreurs et des faiblesses. 


\section{Comment utiliser ce manuel}

Ce livre est un outil de travail pour aider à la mise en oeuvre des études d'AS. Il n'est pas fait pour être lu de bout en bout. Certaines des sections de ce manuel pourraient être utiles et intéressantes pour les décideurs et responsables de programmes, d'autres sections pour les chercheurs, et encore d'autres pour les enquêteurs de terrains. Chaque chapitre du manuel est décrit brièvement cidessous.

\section{Chapitre 1 : Méthodologie de l'étude} d'Analyse Situationnelle. Il sert d'introduction et fournit une base à la méthodologie de la recherche ainsi qu'à une variété de concepts y afférent. Ce chapitre, dont des versions ont été publiées ailleurs, donne une vision globale des objectifs de l'AS, du plan de recherche, de la formation des enquêteurs, des procédures de collecte des données sur le terrain, de l'utilisation des données ainsi que des questions d'ordre méthodologique. Cette information peut être utile aux chercheurs, aux évaluateurs, aux responsables de programmes, aux décideurs politiques, aux bailleurs de fonds et au personnel d'assistance technique travaillant dans les institutions à travers le monde. Ce chapitre a aussi pour but d'aider les décideurs à comprendre l'importance et les limites du type de données collectées, et de les aider à faire un choix éclairé sur la décision ou non de réaliser une telle étude.

\section{Chapitre 2 : Exécution de l'étude. Ce} chapitre fournit une orientation pratique pour la mise en oeuvre de l'étude sur le terrain. Il porte essentiellement sur la formation des agents de terrain, et sera d'un intérêt particulier pour le personnel chargé de l'exécution de l'étude.
On peut faire des photocopies de ce chapitre et les distribuer aux enquêteurs de terrain et aux stagiaires. Il pourrait aussi aider les planificateurs de programmes et les décideurs à comprendre les étapes pratiques dans l'exécution de l'étude.

Chapitre 3 : Instruments et guides question par question. Ce chapitre présente les principaux questionnaires d'interviews, guides d'inventaire et d'observation que les planificateurs peuvent adapter aux intérêts nationaux ou régionaux particuliers. Cette section fournit aussi des instructions et des informations de base sur chaque question, à l'intention des agents de collecte des données. Après l'adaptation des instruments guides question par question qui s'y rattachent, cette section devrait être photocopiée et mise à la disposition de tout le personnel de terrain impliqué dans l'étude. Elle servira d'aide principale pendant la formation des agents de collecte de terrain et aussi d'important document de ressource pédagogique clé .

\section{Chapitre 4 : Analyse des données et} rédaction des rapports. Ce chapitre donne des conseils sur la saisie et le nettoyage des données, et des instructions pour la production d'un rapport préliminaire d'AS qui sera discuté par les chercheurs, les responsables de programmes, le personnel clinique, les bailleurs de fonds et les autres personnes concernées. Il donne une liste complète des résultats préliminaires qui peuvent être calculés à partir des instruments de l'AS, avec référence aux questions nécessaires pour chaque résultat. Il inclut aussi plusieurs échantillons de graphiques et des listes d'échantillons d'indicateurs de deux pays. 
Il importe que les responsables de programmes et les chercheurs se mettent d'accord sur les plans d'analyse avant le démarrage de l'étude. Lorsqu'on arrivera à un consensus, cette section sera utile pour ceux qui sont chargés spécifiquement de l'analyse des données.

Ce chapitre suppose au moins un minimum de maîtrise des programmes de traitement des données (tels que Epi Info ou SPSS). 


\section{La méthodologie de l'étude d'Analyse Situationnelle}

Les programmes de planification familiale ont évolué au cours des trente dernières années. Ils se sont transformés en structures organisationnelles complexes nécessitant des informations détaillées, sur le fonctionnement et la qualité de la prestation des services des composantes telles que la formation, la supervision, la logistique et l'information, l'éducation et la communication (IEC).' L'expansion rapide de ces programmes a souvent exercé de fortes pressions sur les responsables de programmes à connaître le fonctionnement de ces composantes, et ce qui devrait être fait pour en améliorer la performance et accroître les services. Malheureusement, les programmes nationaux de planification familiale manquent souvent de systèmes d'information de gestion (SIG) viables qui pourraient fournir quelques informations pour orienter les décisions relatives à la gestion. Comme cela a été bien documenté par Keller (1991), la plupart des programmes de SMI/PF disposent de médiocres SIG, et dans les programmes qui sont dotés de SIG, "l'information porte très rarement sur les prises de décisions relatives à la gestion" (Keller, 1991:19).

Cette situation explique en partie le fait que les responsables de programmes se soient fiés aux enquêtes basées sur les populations comme source d'information pour orienter le développement des programmes. La nature omniprésente de ces enquêtes ainsi que les nombreux problèmes qui les caractérisent sont connus depuis fort longtemps (Cleland, 1973). Généralement ces enquêtes portent le titre de CAP-Connaissance, Attitude et Pratique. Quelque soit le nom, elles ne peuvent au plus que fournir une information sur la capacité des couples à utiliser ou à ne pas utiliser de méthodes contraceptives (leurs connaissances et attitudes) et sur leur utilisation présente et passée (leurs pratiques). Les enquêtes CAP ne fournissent pas d'information sur le potentiel de l'environnement de prestation de services à fournir des services de qualité aux client(e)s, ou sur la prestation réelle de services aux client(e)s. En termes clairs, elles se focalisent sur la demande de services, non pas sur l'offre des services.

Comme guide de planification de programmes et d'amélioration de services, les enquêtes CAP peuvent ne pas être d'une aide appréciable. En effet, on peut soutenir qu'elles ont eu tendance à mettre en exergue les connaissances, les attitudes et les pratiques des client(e)s comme source de problèmes cherchant à atteindre des buts démographiques, plutôt qu'à se focaliser sur la disponibilité, l'accessibilité et la qualité des programmes comme source de problèmes pour les client(e)s cherchant à atteindre leurs propres objectifs en matière de santé de la reproduction. A titre d'exemple, les faibles niveaux de connaissance de la planification familiale et les attitudes défavorables sont souvent attribués aux valeurs traditionnelles des clientes et aux faibles niveaux d'éducation, et moins fréquemment à la mauvaise planification et à l'exécution des activités IEC, ou à de mauvais conseils donnés comme résultat d'une mauvaise formation du prestataire. De même, les faibles niveaux d'utilisation des contraceptifs sont souvent attribués à un manque de communication entre les conjoints, à une opposition de l'homme à la planification familiale

\footnotetext{
' Ce chapitre est basé sur le document de A. Fisher, R. Miller, I. Askew, B. Mensch, A. Jain, et D. Huntington intitulé "The Situation Analysis approach to assessing the supply side of family planning programs," Population Council, septembre 1994. Une version abrégée et modifiée a été publiée dans The Journal of the Center for African Family Studies, à paraître, 1997.
} 
ou à des valeurs religieuses, et moins fréquemment à un manque de produits, à de véritables barrières à l'accès aussi bien par les hommes que par les femmes, ou à une piètre qualité des services dans les cliniques.

Dominées en grande partie par les recherches opérationnelles, la plupart des recherches en planification familiale se sont focalisées au cours des dernières années sur l'examen des facteurs qui affectent la prestation des services. Une méthodologie de recherches opérationnelles assez standard (Fisher et al., 1991) a été utilisée pour générer un grand nombre d'études (Ross et Frankenberg, 1993; Fisher, 1993) qui examinent le fonctionnement des programmes et les activités ou "interventions" qui sont sous le contrôle des responsables de programmes et qui peuvent être manipulées par le biais d'une action administrative dans le but de produire des changements dans les programmes. Quoique les facteurs de demande constituent clairement d'importants déterminants de l'utilisation des contraceptifs, maintenant il est de notoriété publique que l'efficacité des programmes de planification familiale dépend au moins en grande partie de la disponibilité d'une gamme de produits pour le choix des clientes, de la bonne formation et de la supervision de prestataires compétents, de la compréhension des besoins de la cliente pour un échange efficace de l'information entre prestataire et cliente, de l'encouragement à continuer l'utilisation de méthodes contraceptives, et des attitudes des prestataires qui affectent leur relation avec les clientes et la prestation de services. Ces aspects sont largement perçus comme les éléments fondamentaux de la qualité des soins (Bruce, 1990) et l'on pense qu'ils ont un impact important sur la capacité des couples à atteindre leurs intentions en matière de procréation.

Le développement et l'évolution subséquente de la méthodologie de l'Analyse Situationnelle pour évaluer le volet équipement des programmes de planification familiale a commencé avec une demande d'assistance adressée au Projet de Recherche Opérationnelle en Afrique pour déterminer les équipements nécessaires dans les points de prestation de services (PPS) au Kenya (Miller et al., 1991). A la suite des discussions, la dernière approche de recherche adoptée fut élargie pour inclure une information plus complète sur la disponibilité, le fonctionnement et la qualité des services dans les PPS. Un sentiment général, selon lequel les enquêtes CAP à elles seules ne pouvaient pas servir d'orientation pour la sélection des options du programme, a servi d'important catalyseur pour qu'on se focalise sur l'information relative au volet offre de services. Par exemple, malgré les nombreuses enquêtes CAP au moment de la première étude au Kenya, il n'y avait aucune information disponible sur le potentiel ou la capacité réelle des points de prestation de services à offrir des services. On ne savait pas si les PPS étaient bien équipés et dotés en personnel. On ne savait pas non plus si le matériel IEC était disponible et utilisé. On ignorait aussi si les produits contraceptifs étaient disponibles en quantités suffisantes. En dehors de ces aspects relatifs à la capacité d'un PPS à offrir des services, il n'y avait qu'une information anecdotique sur la qualité des soins que les clientes recevaient en réalité.

Depuis fort longtemps, on connaît l'importance des facteurs qui affectent l'environnement de la prestation des services et la qualité des soins (Freedman, 1974). Les deux enquêtes-Enquête Mondiale Fécondité (EMF) et la dernière Enquête Démographique et de Santé (EDS)—avaient un module sur la communauté, appelé Module de Disponibilité des Services (MDS) par l'EDS. Ce module avait pour but de recueillir l'information sur les services et les installations disponibles dans une zone géographique. Cependant, l'expérience avec les modules sur la communauté n'a pas toujours été perçue comme satisfaisante (Casterline, 1985). Ceci s'explique en partie par la confiance accordée aux informateurs avertis comme source d'information fiable sur les installations et les services. Wilkinson (1991) a démontré que les informateurs avertis ne sont pas toujours bien informés. Ils ne sont pas souvent informés de la 
gamme de services disponibles dans les structures sanitaires ou encore ignorent même l'existence de structures particulières dans une zone géographique donnée. Comme discuté ci-dessous, l'approche de l'Analyse Situationnelle évite l'utilisation des informateurs avertis et se fie plutôt aux interviews avec les prestataires et les clientes doublées d'observations directes des installations et des services faites par les chercheurs formés de l'étude. Cette approche semble déboucher sur des données plus complètes et précises (ArendsKuenning et al., 1996).

\section{Objectifs d'une étude}

\section{d'Analyse Situationnelle}

L'approche de l'Analyse Situationnelle utilisée pour évaluer l'offre des programmes de planification familiale vise quatre principaux objectifs :

1. Décrire le potentiel des politiques actuelles et les standard de programmes à promouvoir la prestation des services de qualité aux clientes;

2. Décrire et comparer l'aptitude du personnel de prestation de services et des structures sanitaires à fournir des services de qualité aux clientes selon les normes et standard des programmes;

3. Décrire au moment de l'enquête la qualité des services reçus par les client(e)s;

4. Evaluer l'impact de l'offre des services de qualité sur la satisfaction des client(e)s, la dynamique de l'utilisation des contraceptifs,

FIGURE 1. Quatre objectifs des études d'Analyse Sitationnelle

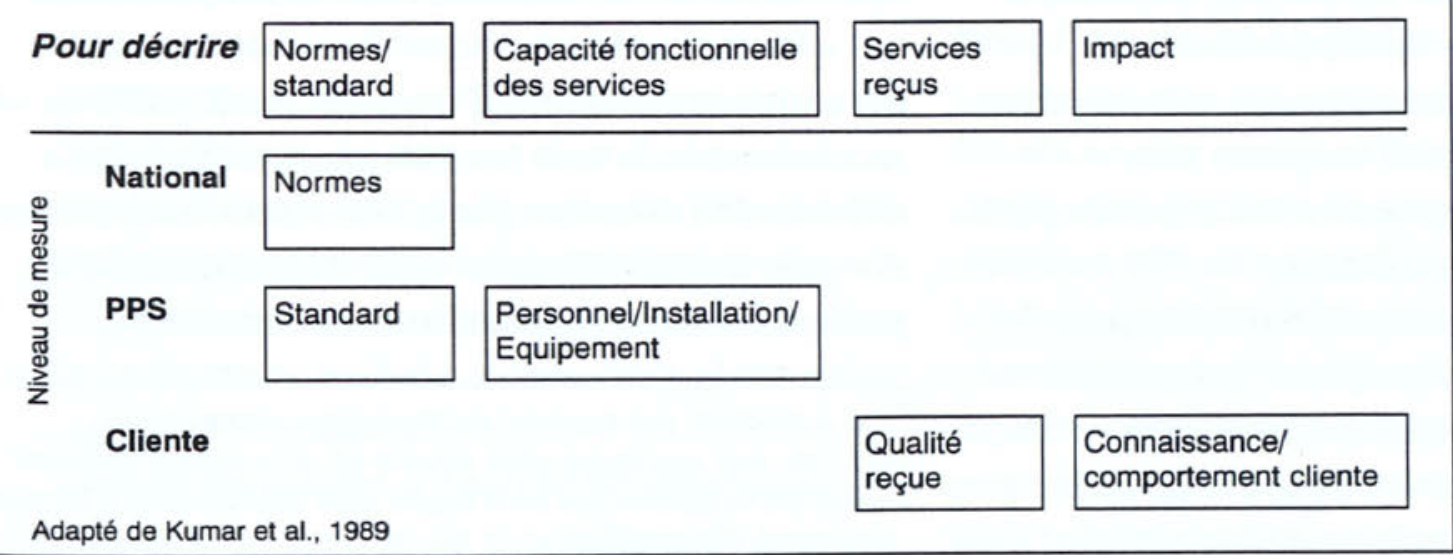

la réalisation des intentions en matière de reproduction et finalement sur la fécondité (utilisant le plus souvent un groupe de répondants).

La figure 1 fait ressortir les liens entre ces quatre objectifs et trois niveaux de mesureniveau national, niveau des PPS, et niveau des client(e)s.

Au fur et à mesure que l'on va de l'objectif 1 à l'objectif 4 , on constate une augmentation concomitante dans la complexité du plan d'étude, dans les procédures de collecte des données, ainsi que dans l'analyse des données. Parallèlement, on constate une augmentation du temps et des coûts nécessaires pour l'exécution de l'étude. Par exemple, l'étude suggérée par l'objectif 1 est premièrement de nature descriptive et vise à fournir l'information sur le degré auquel les normes et les standard des services PF ont tendance à faciliter et à promouvoir la prestation des services de qualité aux client(e)s ou à agir comme des barrières. Les normes et les standard au niveau des PPS concernent une variété de problèmes tels que les taxes à l'importation sur les contraceptifs, le coût des services, les clientes éligibles pour les méthodes contraceptives, les méthodes qui devraient être disponibles dans tous les PPS, les compétences que le personnel devrait avoir, les informations qu'on devrait donner aux clientes, etc. (Bien que toutes les AS n'incluent pas cet objectif, il existe un questionnaire d'interview d'AS à l'intention des responsables de programmes qui a été utilisé dans plusieurs sites.)

Le deuxième objectif propose une description de la situation qui prévaut 
dans les PPS. Généralement, une étude d'Analyse Situationnelle de "base" décrit "l'aptitude" du système de prestation de services à offrir des services de qualité. Au niveau du PPS, la capacité à offrir services de qualité signifie : que le personnel est disponible, formé à offrir des services et compétent; que les produits et les équipements sont disponibles, en état de fonctionnement et sont utilisés; et que la structure est capable de traiter de manière adéquate un grand nombre de client(e)s. Autrement dit, que les conditions pour une prestation de services de qualité existent.

Le troisième objectif propose une étude plus analytique qui 1) décrit la qualité des services reçus par la cliente, c'est-à-dire l'interaction entre le prestataire et la cliente, et 2) fait une comparaison du potentiel du programme à fournir des services de qualité par rapport à la qualité réelle des services reçus par les client(e)s. Par exemple, une clinique qui ne dispose pas de produits contraceptifs, de personnel formé, et des équipements de base n'a pas le potentiel de fournir des services de qualité. Une telle clinique n'est pas prête à offrir des services de planification familiale de quelque nature que ce soit, à plus forte raison des services de qualité. D'autre part, la présence de produits contraceptifs, du personnel formé et d'un équipement de base n'est pas une garantie que les clientes reçoivent des services de qualité. Un prestataire, même s'il est très bien formé, peut ne pas vouloir offrir des services aux adolescentes célibataires, offrir une gamme complète de méthodes aux clientes, faire une description complète de chaque méthode, discuter des effets secondaires des méthodes, encourager les clientes à changer de méthodes en cas de problèmes avec la méthode utilisée, discuter des questions relatives aux MST, ou poser des questions pour bien comprendre les objectifs de la cliente en matière de procréation.

Le quatrième objectif qui a trait à l'impact propose une étude assez complexe qui pourrait utiliser un procédé de recherche prospectif, longitudinal pour collecter les données au fil du temps pour tester une ou plusieurs hypothèses relatives à "l'impact" qu'une organisation particulière de services ou niveau de la qualité a sur la satisfaction des clientes, l'utilisation des contraceptifs et la capacité des clientes à réaliser leurs propres intentions en matière de procréation de manière sûre et saine. L'étude de l'impact nécessite qu'une étude d'AS soit liée à une étude de type CAP basée sur la population, soit qu'au moins deux études d'Analyse Situationnelle de base soient réalisées au fil du temps, et qu'un groupe de répondants soit suivi d'une étude à la suivante (voir la section ci-dessous).

\section{Eléments de base}

Le développement de la méthodologie de l'Analyse Situationnelle a été influencé par l'approche de PRICOR (Center for Human Services, 1988), par la Méthodologie de l'Enquête Rapide de Frerichs (Frerichs, 1989a, et Frerichs et Khin Tar Tar, $1989 b)$ et par le modèle de la qualité des soins de Bruce et Jain (Bruce 1990). En développant cette méthodologie, plusieurs hypothèses ont été émises : 1) Il serait important d'avoir une image du fonctionnement de toutes les principales composantes de planification familiale plutôt que de se concentrer sur une ou deux, telles que l'équipement ou l'IEC. 2) La corrélation entre la réalisation de différentes tâches ou sous-tâches au sein d'une composante particulière était probablement tellement forte que la collecte de données sur un groupe limité d'indicateurs de composantes au lieu de tous les indicateurs serait peut-être suffisante pour déterminer si la composante fonctionne. ${ }^{2}$ 3) Pour faire des déductions sur les facteurs relatifs aux provisions qui influent sur la prestation de services, soit un échantillon représentatif des PPS serait visité ou un recensement de tous les PPS serait réalisé. 4) $\mathrm{La}$ collecte des données par le biais des observations directes des installations et du comportement du personnel, aussi bien que par des interviews

\footnotetext{
${ }^{2}$ On a préféré, par rapport au Thesaurus complet de PRICOR, une approche plus simple et plus rapide utilisant moins de variables, qu'on a jugée plus convenable à l'étude d'un vaste échantillon.
} 
auprès des prestataires de services dans les PPS et auprès de leurs clientes donnerait une information plus riche et plus valide que celle obtenue par des interviews réalisées auprès des informateurs clés.

L'approche de recherche qui s'est développée à partir de l'étude du Kenya fut appelée Analyse Situationnelle que l'on définit comme :

Une description et une évaluation:

1) des normes et standard de prestation de services

$\mathrm{PF}$; et la disponibilité ainsi que du fonctionnement des composantes de planification familiale au sein d'un échantillon représentatif ou au niveau de tous les points de prestation de services (PPS) dans une zone géographique donnée; 2) de la capacité de ces composantes à fournir des services de qualité aux clientes; 3) de la qualité réelle des soins reçus par les clientes dans ces PPS; et 4) de l'impact de la qualité des soins sur le comportement de la fécondité des clientes.

Mensch et al. (1994a) ont constaté que:

"Bien que l'Analyse Situationnelle ait emprunté aux autres méthodologies, elle est considérée comme innovatrice parce qu'elle intègre un nombre d'approches à l'évaluation du programme de planification familiale. Ces approches incluent: (1) un système pour identifier les éléments des composantes clés du fonctionnement du programme; (2) des visites d'un grand échantillon de PPS plutôt que se fier à l'opinion d'experts; (3) une attention accordée à la qualité des soins qui tient compte des client(e)s; (4) des interviews structurées auprès des responsables, des prestataires et des client(e)s plutôt qu'avec les informateurs communautaires comme c'est le cas avec le module de disponibilité de I'EDS; (5) l'inventaire des installations cliniques, des équipements et des produits disponibles le jour de la visite de l'équipe; (6) l'observation directe non participative de toutes les interactions cliente prestataire de planification familiale le jour de la visite de l'équipe de recherche."

Toutes les études d'Analyse Situationnelle possèdent un ensemble de procédures de base et d'approches standards pour collecter les données.

Ces procédures et approches incluent:

- Un échantillon représentatif de PPS, ou tous les PPS dans une zone géographique d'intérêt particulier (pays, ville, district, province) sont visités au moins pendant une journée entière par une équipe composée d'au moins trois personnes, dont au moins une ayant une formation clinique (médecin, infirmier, infirmière/sage-femme) et au moins une ayant une formation en sciences sociales avec une expérience d'interviews sur le terrain.

- Un inventaire complet des équipements et des provisions

- Un enregistrement des statistiques des 12 derniers mois (s'ils sont disponibles).

- Une interview de tous les prestataires de planification familiale sur les questions de planification familiale et autres problèmes de santé de la reproduction.

- Des observations sur les interactions entre les prestataires de services et toutes les nouvelles et anciennes clientes de planification familiale présentes le jour de la visite.

- Une interview de toutes les clientes observées au moment où elles quittent le PPS. Une sélection de clientes SMI sont aussi interviewées.

Certaines études d'AS incluent aussi des interviews avec les responsables de programmes, des observations des services autres que ceux de la planification familiale, ainsi que des questionnaires spécialisés pour la DBC et les pharmacies.

\section{Procédé de recherche}

Les unités d'analyse d'une étude d'Analyse Situationnelle sont les PPS. Au lieu d'une seule clinique ou système de prestation de services, plusieurs PPS et types de systèmes de prestation (cliniques du MS, DBC, cliniques des ONG, pharmacies, etc.) représentatifs de la situation d'un pays ou d'une région sont examinés. Les PPS peuvent inclure les cliniques gouvernementales et para-publiques ainsi que les réseaux $D B C$, les 
TABLEAU 1. Tailles des échantillons des études d'Analyse Sitationnelle sélectionnées

\begin{tabular}{|c|c|c|c|c|c|c|}
\hline Pays & Année & $\begin{array}{l}\text { Nombre } \\
\text { de PPS }\end{array}$ & $\begin{array}{l}\text { Observation } \\
\text { prestataire- } \\
\text { cliente }\end{array}$ & $\begin{array}{l}\text { Interviews } \\
\text { des clientes } \\
\text { PF }\end{array}$ & $\begin{array}{c}\text { Interviews } \\
\text { avec le } \\
\text { personnel }\end{array}$ & Agence d'exécution \\
\hline \multicolumn{7}{|c|}{ Afrique subsaharienne } \\
\hline Botswana & 1995 & 184 & 406 & 386 & 456 & Ministère de la Santé \\
\hline Burkina Faso & 1992 & 53 & 66 & 193 & 93 & Ministère de la Santé \\
\hline Burkina Faso & 1995 & 337 & 509 & 509 & 685 & Ministère de la Santé \\
\hline Côte d'Ivoire & 1992 & 13 & 163 & 355 & 51 & AIBEF \\
\hline Ghana & 1993 & 399 & 665 & 655 & 783 & Ghana Statistical Office \\
\hline Kenya & 1989 & 99 & 72 & 72 & 99 & Ministère de la Santé \\
\hline Kenya & 1995 & 254 & 1029 & 997 & 562 & Ministère de la Santé \\
\hline Naïrobi Ville & 1991 & 46 & 100 & 100 & 46 & Nairobi City Committee \\
\hline Nigeria & 1992 & 181 & 395 & 390 & 289 & Université Obafemi \\
\hline Sénégal & 1994 & 180 & 1123 & 985 & 361 & Ministère de la Santé \\
\hline Tanzanie & 1992 & 348 & 450 & 436 & 537 & Ministère de la Santé \\
\hline Tanzanie (Mbeya) & 1995 & 52 & 111 & 111 & 120 & Projet Santé familiale \\
\hline Zanzibar & 1995 & 100 & 145 & 145 & 191 & Ministère de la Santé \\
\hline Zimbabwe & 1991-92 & 181 & 463 & 463 & 378 & ZNFPC \\
\hline \multicolumn{7}{|l|}{ Asie } \\
\hline Indonésie & 1994 & 342 & 1625 & 1624 & 651 & BKKBN \\
\hline Pakistan & 1993 & 84 & 103 & 203 & 83 & Ministry of Population Welfare \\
\hline Turquie & 1994 & 115 & 1006 & 975 & 232 & Ministère de la Santé \\
\hline \multicolumn{7}{|l|}{ Amérique latine } \\
\hline Ceara, Brésil & 1993 & 40 & 17 & 17 & 47 & Sociedade Cearense Pediatra \\
\hline Guatemala & 1993 & 38 & na & 331 & 71 & Ministère de la Santé/PF \\
\hline Pérou & 1992 & 2970 & 599 & 599 & 1961 & $\begin{array}{c}\text { Ministère de la Santé et } \\
\text { Institut National des Statistiques }\end{array}$ \\
\hline
\end{tabular}

réseaux des ONG, les unités commerciales telles que les pharmacies, les hôpitaux privés, les cliniques privées, et les réseaux des lieux d'emploi (usines, mines ou grandes fermes). En bref, toutes les sources de planification familiale et de services de santé ainsi que les sources potentielles de services PF et de santé pourraient être examinées dans une étude d'Analyse Situationnelle.

Cependant, la première décision à prendre est la suivante : Quel type de PPS devrait-on évaluer? Une fois que cette décision est prise, on pourrait ensuite décider d'inclure la totalité des PPS dans l'étude ou plus probablement, de développer une stratégie d'échantillonnage.

Si l'objectif visé par l'Analyse Situationnelle est de diagnostiquer et de décrire la disponibilité, le fonctionnement et la qualité des services dans tout le pays, alors l'échantillonnage des PPS est presque toujours nécessaire. L'idéal est que l'échantillon soit représentatif des PPS en termes de type (hôpital, clinique, DBC, etc.) de services offerts (planification familiale seule ou intégrée à la santé de la reproduction), de localisation géographique (région, zone urbaine/rurale), et de type de secteur (publique, ONG, privé, etc.). Ceci nécessite une liste détaillée de la localisation de tous les PPS aussi bien qu'une connaissance du type de PPS dans chaque localité. En admettant qu'il existe une liste de tous les PPS, on peut ensuite faire un simple échantillonnage aléatoire ou, plus probablement, une sorte d'échantillonnage aléatoire stratifié, avec les unités tirées proportionnellement au nombre dans une grappe. Généralement, les échantillons sont stratifiés en fonction des classifications mentionnées ci-dessus : région, type de PPS, secteur, etc. Le tableau 1 fait un résumé des tailles des échantillons utilisés dans des études d'Analyse Situationnelle récentes. 


\section{Instruments de collecte des données}

Un certain nombre d'instruments de collecte de données sont nécessaires pour recueillir

l'information sur les trois premiers domaines d'intérêt (disponibilité, fonctionnement et qualité). Certains de ces instruments doivent être utilisés dans toutes les études d'Analyse Situationnelle, sinon le minimum de données requis pour décrire le fonctionnement et la qualité de la composante ne peut pas être obtenu. D'autres instruments sont facultatifs mais peuvent toutefois enrichir les résultats de l'étude lorsqu'ils sont utilisés.

Dans une étude de PPS fixes tels que les cliniques et les hôpitaux, les instruments de base de l'Analyse Situationnelle peuvent être utilisés. L'administration de ces instruments nécessitera approximativement quatre à six heures par PPS cela dépend du nombre de clientes dans le PPS. On ne devrait couvrir qu'un seul PPS par jour, sinon il n'y aura pas assez de temps pour bien examiner chaque composante. Dans certains cas, il pourrait être indiqué de passer plus d'un jour dans chaque PPS (voir les discussions relatives à ce point cidessous). L'équipe de collecte de données devrait être présente dans le PPS quelques minutes avant son ouverture et y rester jusqu'à sa fermeture. Le minimum d'instruments de base pour la collecte de données sont les suivants :

- Inventaire des installations disponibles et des services offerts dans le PPS

- Guide d'observation de l'interaction entre les clientes de planification familiale et les prestataires de services

- Interview de fin de consultation pour les clientes de planification familiale

- Interview du personnel fournissant les services de planification familiale/santé de la reproduction dans les points de prestation de services

- Interview des clientes SMI fréquentant les points de prestation de services

Généralement, comme il y a quelques clientes de planification familiale le jour de la visite, il n'est pas possible de faire une évaluation correcte de la qualité des soins dans un PPS quel qu'il soit. Cependant, regrouper toutes les clientes de toutes les cliniques dans un échantillon peut fournir une conclusion raisonnablement valide sur la qualité des soins d'une région ou d'un pays. Si le but visé est d'obtenir la qualité des soins dans chaque PPS, il faudra dans ce cas recueillir l'information sur un plus grand nombre de clientes. Cette information peut être obtenue de la manière suivante: 1) Passer plus de temps (si possible au moins une semaine) dans chaque PPS ou 2) Stratifier les PPS en composantes qui fonctionnent ou selon le nombre de clientes, sélectionner quelques PPS par grappes, et passer davantage de temps pour collecter les données sur les clientes.

En fonction du système de prestation de services qu'on est en train d'étudier, des instruments supplémentaires peuvent être administrés. Par exemple :

- Questionnaire à l'intention des responsables de programmes et des décideurs

- Plan d'interview DBC

- Formulaire d'interview de la cliente DBC

- Interview de sortie de la cliente DBC

- Questionnaire d'interview pour les pharmacies.

Dans certains PPS, en particulier dans les pays ayant une faible prévalence contraceptive, on peut ne pas trouver suffisamment de nouvelles ou d'anciennes clientes à interviewer. Dans de telles circonstances, le questionnaire des clientes SMI peut aider à déterminer les causes de la non utilisation de services par les femmes qui habitent à proximité du PPS. Si un programme de planification familiale inclut des agents $D B C$ qui fournissent des contraceptifs à un nombre important de clientes, dans ce cas on peut aussi développer et utiliser un instrument $\mathrm{DBC}$. Les instruments $\mathrm{DBC}$ ont été utilisés dans quelques pays (par exemple le Zimbabwe, et le Mali) et sont disponibles auprès du Population Council.

Il existe beaucoup d'autres modules de questionnaires qui peuvent être ajoutés aux questionnaires standard. Par exemple, la méthodologie de l'Analyse Situationnelle peut être utilisée pour examiner les liens (intégration) entre 
les services de santé de la reproduction (postpartum, prénatal, avortement, MST et VIH) et les services de planification familiale. En outre, dans les pays où la stérilisation est importante, on peut ajouter un module qui examine ce service dans les moindres détails. En bref, l'approche de l'Analyse Situationnelle peut être utilisée pour avoir non seulement une image des services de planification familiale mais aussi celle d'autres services connexes. A titre d'exemple, les récentes modifications des instruments de collecte des données ont entièrement couvert les problèmes de santé de la reproduction tels que l'avortement (AS du Sénégal), les services de maladies sexuellement transmissibles (AS du Botswana), et les services de stérilisation (AS de Turquie).

\section{Exécution de l'étude}

Le nombre d'équipes de recherche et de superviseurs utilisés dans une étude dépend du nombre et de l'accessibilité des PPS, des objectifs ainsi que des ressources disponibles.

Généralement, chaque équipe de recherche est composée de trois personnes-au moins une ayant une formation clinique (médecin, infirmier, infirmière/sage-femme) et au moins une ayant une formation en sciences sociales avec une expérience d'interviews sur le terrain. Il est recommandé qu'un seul superviseur ne coordonne pas plus de trois équipes de recherche.

En général, au début de la journée, la personne ayant une formation clinique commencera à observer les interactions entre les clientes et les prestataires. Plus tard, cette même personne aidera un autre membre de l'équipe pour faire l'inventaire de l'équipement. Quant au spécialiste d'enquêtes en sciences sociales, il sera chargé des interviews de fin de consultation des clientes et des interviews du personnel. Il/elle peut aussi aider dans la collecte des informations sur les statistiques de la clinique, la rédaction du rapport et les statistiques de services.

La formation des équipes de recherche est probablement l'aspect le plus important de toute étude d'Analyse Situationnelle. A la différence des enquêtes CAP, les études d'Analyse Situationnelle utilisent une variété de techniques de collecte de données telles que les observations et les inventaires qui nécessitent une bonne formation. Dix journées entières de formation est le minimum requis. La formation devrait inclure aussi bien de nombreux jeux de rôle que de vraies visites dans les PPS pour la pratique (voir "Programme de formation de l'équipe de recherche." page 19).

Avant la formation même des enquêteurs, il est généralement très utile pour les principaux chercheurs et les superviseurs de l'étude d'avoir une orientation précise de l'approche de l'Analyse Situationnelle ainsi que de la gestion de l'étude. Cette orientation devrait se faire pendant quelques jours, voire une semaine. Elle devrait couvrir tous les aspects du processus de collecte des données y compris la formation, la localisation des PPS, l'organisation et la vérification des questionnaires, les procédures de rappel et les dispositions à prendre en cas d'urgence.

\section{Utilisation des études d'Analyse Situationnelle}

Les principaux utilisateurs des résultats d'une Analyse Situationnelle sont les décideurs, les bailleurs de fonds, les responsables de programmes, et les prestataires de services à tous les niveaux (Askew et Fisher, 1995). Le plan d'analyse standard des données d'une étude d'Analyse Situationnelle contient 100 tableaux et graphiques qui sont d'habitude générés de chaque étude (voir chapitre 4), discutés de manière extensive lors d'un séminaire de dissémination, et peuvent enfin figurer dans le premier rapport. L'information obtenue peut être utilisée pour une variété d'objectifs relatifs au programme afin :

- D'évaluer la qualité des soins offerts aux clientes par les cliniques du secteur privé et celles du secteur public et par les DBC;

- D'examiner le degré d'intégration des services de santé de la reproduction dans les programmes de planification familiale;

- De raffiner les estimations des besoins de formation du personnel (initiale et continue); 
- D'examiner la disponibilité et l'utilisation des matériels d'information, d'éducation et de communication (IEC);

- De mettre en exergue les besoins en équipement, en particulier pour les méthodes cliniques telles que le DIU et la stérilisation;

- De guider les plans de rénovation des installations;

- De quantifier les niveaux actuels de provisions de contraceptifs dans les points de prestation de services visités;

- D'évaluer le fonctionnement du SIG et de la logistique;

- D'évaluer les barrières à l'accessibilité;

- De fournir des données en vue de la formulation ou du changement de politique.

- D'identifier les thèmes pour initier des études de recherche opérationnelle.

La plupart des études d'Analyse Situationnelle récentes sont utilisées maintenant pour évaluer des aspects de santé de la reproduction tels que les services relatifs à l'avortement, les MST et le VIH/SIDA. D'autres ont utilisé l'approche de l'Analyse Situationnelle pour examiner les soins obstétricaux d'urgence (Center for Population and Family Health, 1995; et Sloan et al., 1995). Par exemple, on peut poser des questions au personnel des PPS sur l'accès aux services d'avortement, et s'enquérir des questions qu'ils ont reçues des clientes sur l'avortement. On peut leur poser aussi des questions sur la disponibilité des équipements, des produits et d'un personnel formé pour fournir des avortements sans risques et des services de soins obstétricaux d'urgence. De même, on peut demander aux clientes si elles n'ont jamais eu de grossesse non désirée, et si oui, comment elles ont géré cette grossesse. Cette approche a été utilisée dans bon nombre d'études d'Analyse Situationnelle (voir Huntington et al., 1993a et Huntington et al., 1996b).

Si une étude d'Analyse Situationnelle couvre tous les PPS d'une zone géographique telle qu'une ville ou un pays (voir Mensch et al., 1994b pour le cas de Naïrobi), les faiblesses de tout PPS pris dans cette zone géographique en termes de manque de personnel, d'équipement, de provisions ou d'autres éléments peuvent être identifiées facilement et souvent corrigées rapidement. En outre, le système pris dans son ensemble peut être décrit avec précision puisque tous les PPS sont inclus dans l'analyse. Cependant, si une approche d'échantillon est adoptée pour la sélection des PPS, il est possible de décrire tout le système de prestation de services en termes de probabilités et non pas en termes des faiblesses d'un PPS donné.

Un modèle d'exécution des études d'Analyse Situationnelle est apparu et inclut la participation des responsables de programmes dans toutes les phases clés de l'exécution de l'étude afin d'améliorer la compréhension du processus de recherche et l'utilisation des résultats de l'étude pour les changements à faire dans le programme. D'abord, une réunion de planification ou d'orientation est organisée et à laquelle participent les chercheurs, les responsables des PPS à visiter, et les principaux utilisateurs des données (tels que les décideurs et les bailleurs de fonds). La méthodologie et les procédures de l'Analyse Situationnelle sont expliquées en détail au cours de cette réunion. En plus, le plan d'échantillonnage est développé pour refléter les besoins de toutes les organisations de planification familiale pertinentes du pays, les principaux instruments de collecte de données sont adaptés aux systèmes spécifiques de prestation de services en cours d'analyse, et les responsables s'accordent sur les responsabilités pour assurer l'accès de l'équipe de recherche aux PPS. Les principaux haut cadres du programme continuent à être impliqués dans les activités y compris les phases de formation et de collecte des données sur le terrain.

Une fois que les données ont été collectées et traitées, un atelier d'interprétation de données est organisé par le même groupe de responsables, de décideurs et de bailleurs de fonds pour discuter les résultats préliminaires de l'étude. La saisie rapide des données et la production des tableaux de fréquences des variables, avec quelques tableaux 
de base croisés là où cela s'avère nécessaire, sont des activités nécessaires pour ce séminaire (voir chapitre 4). Cette production rapide des premières données est facilitée par des programmes informatiques simples à utiliser tels que Epi Info (Dean et al., 1990). Les résultats sont présentés généralement sous forme de diagrammes en bâton pour chaque variable. Les diagrammes en bâton sont assez faciles à comprendre par toutes les audiences et semblent stimuler les discussions et la recherche de solutions aux problèmes qui surgissent. L'un des principaux objectifs du séminaire d'interprétation des données est de permettre aux responsables de programmes de voir les données préliminaires avant la rédaction d'un rapport final et avant qu'une analyse ou interprétation ne soit faite de ces données. De cette manière, l'interprétation des données relève de leur compétence et non pas de celle des chercheurs. Un objectif principal de ce séminaire est d'obtenir un consensus sur les implications programmatiques des données.

Après le séminaire d'interprétation des données, un rapport final est rédigé et inclut les recommandations visant à améliorer et à porter des changements dans le programme. Ce rapport final est présenté lors d'un ou de plusieurs séminaires de dissémination où les résultats sont discutés par les participants. Les récentes études d'Analyse Situationnelle réalisées au Sénégal, au Burkina Faso et en Indonésie ont porté essentiellement sur la dissémination des résultats non seulement au niveau national, mais aussi au niveau régional afin d'obtenir une implication maximale des responsables de programmes au niveau local et d'accroître la probabilité pour que les résultats soient utilisés pour produire un changement positif au niveau du programme (Diop et al., 1996).

\section{Etudes d'impact et Analyse Situationnelle}

Les études d'impact se réalisent à partir du plan de base de l'Analyse Situationnelle et répondent au quatrième objectif mentionné auparavant. Il existe trois différentes études d'impact qui peuvent être entreprises. La première est la plus simple et peut être appelée plus précisément une étude d'évaluation de "résultat" plutôt qu'une étude d'impact. ${ }^{3}$ La procédure à suivre serait de réaliser deux études d'Analyse Situationnelle dont chacune utiliserait le même échantillon de PPS. La deuxième étude serait réalisée approximativement au moins trois ans après la première. L'objectif serait d'évaluer les changements qui se seraient produits comme résultat des améliorations du programme. "L'impact" de ces changements serait mesuré non seulement en termes du potentiel des composantes des PPS améliorés à offrir des soins de qualité, mais aussi en termes de qualité des soins réellement reçus par les clientes (Miller et Frerichs, 1992-93; et Miller et al., 1996).

Un second type d'étude d'impact serait de type longitudinal et nécessiterait aussi bien la conduite de deux études d'Analyse Situationnelle dans le même échantillon de PPS que la création d'un groupe de suivi de clientes. Dans la première étude, les intentions des clientes en matière de procréation seraient déterminées lors des interviews de sortie. Ces clientes formeraient un groupe de suivi et seraient réinterviewées à peu près deux à trois ans plus tard. Pendant cette période, il se pourrait que les problèmes identifiés dans les PPS par la première étude d'Analyse Situationnelle soient éliminés ou au moins réduits par une forme de programme d'intervention de prestation de services. ${ }^{4}$ Une seconde Analyse Situationnelle de suivi serait ensuite réalisée pour déterminer le degré d'amélioration du potentiel de

\footnotetext{
${ }^{3}$ Voir Bertrand et al., 1994, p. 19. Bertrand et ses collègues ébauchent un processus de :

INTRANTS-> PROCESSUS ->EXTRANTS ->RESULTATS où les intrants, le processus et les extrants réfèrent aux composantes spécifiques du projet ou du programme, et les résultats aux "résultats ultimes" plus vastes basés sur la population et qui ont trait à l'effet et à l'impact.

${ }^{4} \mathrm{Si}$ une intervention n'est pas possible, alors il serait nécessaire de sélectionner un environnement géographique où il y a des changements dans les ressources de planification familiale, y compris la qualité des services. En l'absence de changement, il ne serait pas possible de démontrer un lien entre la qualité des soins, l'utilisation contraceptive et la fécondité.
} 
capacité des composantes du PPS et de la qualité des soins. Si la capacité fonctionnelle et la qualité ont été améliorées de manière significative, alors l'impact de cette amélioration devrait être perceptible dans le comportement du groupe de suivi en ce qui concerne leur capacité à satisfaire leurs propres intentions en matière de procréation. Ce type d'étude examine essentiellement le degré auquel les différences dans le comportement contraceptif peuvent être attribuées aux différences dans la qualité et la configuration des services offerts dans les PPS.

Le troisième type d'étude d'impact vise un objectif similaire. Il viserait à établir un lien entre une étude d'Analyse Situationnelle et une étude de type CAP basée sur la population telle qu'une enquête démographique et de santé (EDS). Tous les PPS de la zone géographique couverts par l'enquête basée sur la population seraient inclus dans l'Analyse Situationnelle. La configuration particulière et la qualité des services offerts par les PPS pourraient ensuite être attribuées au comportement contraceptif des couples qui ont été échantillonnés et interviewés dans les zones géographiques desservies par les PPS (voir Mensch et al., 1996).

\section{Recherche opérationnelle et Analyse Situationnelle}

Les études d'Analyse Situationnelle sont étroitement liées aux recherches opérationnelles et en constituent une composante essentielle de son processus. Comme l'ont défini Fisher et al. (1991), le processus de la RO comporte cinq étapes principales : identification et diagnostic du problème, sélection de la stratégie, expérimentation et évaluation de la stratégie, dissémination de l'information, et utilisation de l'information. Les études d'Analyse Situationnelle sont devenues l'un des outils les plus importants pour rassembler les données nécessaires à la première étape du processus RO-identification et diagnostic du problème.

Les études d'Analyse Situationnelle identifient et définissent dans les moindres détails les contraintes à la prestation de services et qui sont relatives à l'offre. Une fois le problème identifié, les études RO peuvent ensuite être conçues et exécutées pour tester les nouvelles approches visant à le surmonter. Les résultats de ces études sont ensuite disséminés et utilisés pour améliorer les services. Après que les résultats aient été utilisés, une deuxième étude d'AS peut examiner les changements intervenus dans l'environnement de la prestation de services depuis la première étude, et l'impact que ces changements ont pu avoir sur le comportement de la fécondité des clientes. La seconde étude d'AS sert aussi à identifier de nouveaux problèmes qui auraient pu se développer ou d'anciens problèmes qui n'ont pas été résolus. Ainsi, non seulement les études d'AS débutent le processus de recherches opérationnelles avec l'identification et le diagnostic du problème, et le terminent par l'évaluation des changements et de l'impact, mais aussi elles commencent un nouveau cycle d'identification et de diagnostic de problème comme le montre la figure 2 .

\section{La diffusion des études d'Analyse Situationnelle}

Les résultats de la première étude du Kenya ont très vite été mis à la disposition des responsables de programmes sous forme de graphiques (Miller et al., 1989) ce qui a facilité énormément leur compréhension en même temps que leur dissémination. Cela a aussi favorisé la diffusion rapide de la méthodologie. Les données se sont révélées utiles aux responsables du programme de planification familiale du Kenya qui ont pensé que pour la première fois, ils ont mieux compris l'offre de leur programme et pouvaient le présenter au Gouvernement et aux bailleurs de fonds.

Quelques changements spécifiques ont été faits dans la planification et la gestion du programme dans le but de résoudre les problèmes rapidement. Les données ont été utilisées aussi pour former le personnel sélectionné du MS et des universités locales en RO, et pour concevoir et exécuter plusieurs études $\mathrm{RO}$ visant à résoudre les problèmes identifiés par l'étude d'AS. 
FIGURE 2. Relation entre le Processus de la Recherche Opérationnelle et de l'Analyse Situationnelle

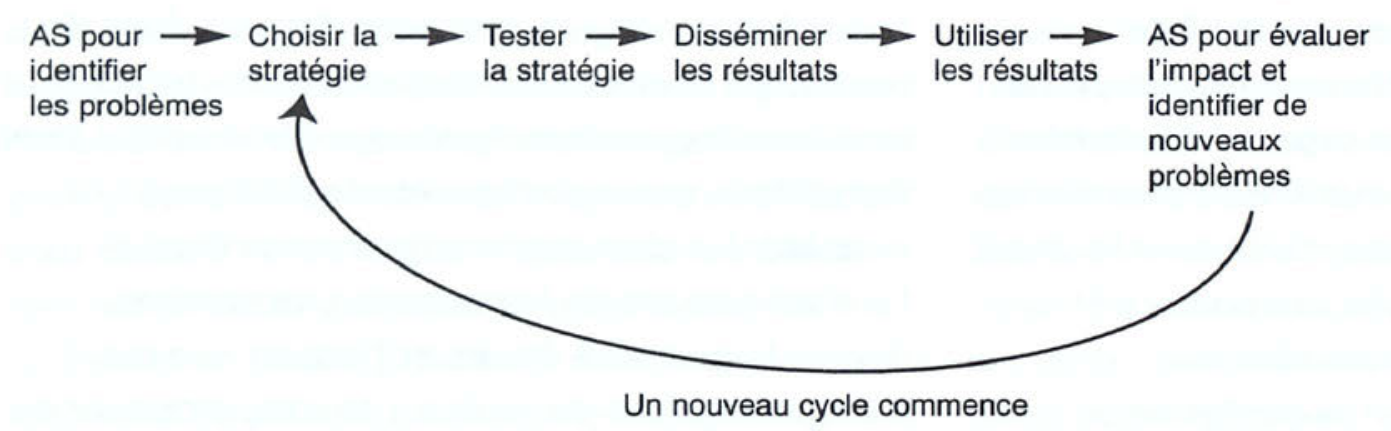

façon positive dû à la présence de l'observateur. Les réponses obtenues de l'interview du personnel sont probablement aussi biaisées positivement en ce sens qu'elles ont tendance à présenter le fonctionnement des PPS de manière

L'intérêt suscité par la première étude d'AS et ses efforts de dissémination ont occasionné de nombreuses demandes pour d'autres études, d'abord en Afrique et plus tard dans les pays d'Asie et d'Amérique latine. Vers la fin de 1996, plus de 35 études avaient été achevées ou étaient en cours d'achèvement dans le monde entier, et un plus grand nombre était à différentes étapes de planification. Plus récemment, l'utilisation des résultats d'études d'Analyse Situationnelle a été documentée dans un nombre d'études de cas au Burkina Faso (Population Council, 1996).

\section{Problèmes d'ordre méthodologique}

Dans le processus de planification et de réalisation des études d'AS, trois facteurs sont à la base de l'évolution de la méthodologie: (1) adaptation aux intérêts locaux et aux composantes du programme; (2) analyse critique par un plus grand nombre de chercheurs impliqués dans les études; et (3) le désir d'explorer davantage de solutions possibles aux questions et problèmes d'ordre méthodologique persistants. Plusieurs questions d'ordre méthodologique dont certaines ont été reconnues depuis la première étude du Kenya sont devenues plus apparentes et devraient être résolues dans le futur. Ces questions comprennent :

- Biais positif de l'observation et de l'interview-Alors que les données collectées à l'aide du questionnaire de "l'inventaire des PPS" comportent peu de biais, les observations de l'interaction cliente prestataire sont probablement biaisées de favorable. Les chercheurs ont été préoccupés par ces problèmes depuis la première étude. Une solution probable à ce problème est de passer plus d'un jour dans chaque PPS afin que la présence de l'équipe de recherche redevienne plus naturelle et que le comportement des prestataires devienne plus normatif (Simmons et Elias, 1993). Une autre approche visant à atténuer le biais positif pourrait être l'utilisation de clientes "simulées" (Huntington et Schuler, 1993b).

- Fiabilité des observations-L'observation de l'interaction prestataire-cliente constitue une importante source des données des études d'AS. La fiabilité constitue une importante préoccupation avec ces données. Bien que les observateurs reçoivent une formation intensive, il est évident qu'il existe un degré de variabilité entre eux. Dans l'étude d'AS de Turquie, deux observateurs ont enregistré la même interaction prestataire-cliente. Une comparaison des réponses des deux observateurs a révélé de manière globale un degré très élevé de fiabilité (Huntington et al., 1996a). Quoique ce résultat soit satisfaisant, davantage d'études méthodologiques de ce type doivent être réalisées.

- Le nombre et la représentativité des PPS et des observations-Il y a un nombre important de problèmes d'échantillonnage liés aux études d'AS. D'abord, il y a le problème de connaissance du nombre de PPS à inclure dans 
l'échantillon. Quel est le type de PPS qui devrait être inclus-seulement les PPS du MS ou un mélange avec les PPS privés ? Des échantillons séparés devraient-ils être inclus pour chaque type de PPS ? Aussi, combien d'interactions prestataire-cliente devraient être observées dans chaque PPS ? Cette question devient plus importante parce que les nouvelles clientes ne sont pas souvent disponibles dans les PPS le jour de la visite, et comme la présence des clientes est moins probable dans les PPS de qualité inférieure, alors les observations qui résultent des interactions prestataires-clientes biaisent davantage l'image de tout le programme de manière positive. Il y a aussi la question de savoir si les différents types de PPS (par exemple, hôpitaux urbains, centres de santé de district, ou postes de santé villageois) devraient être évalués en termes de fréquentation de clientes ou si possible selon la population de la circonscription hospitalière.

- Besoins de recherche appliquée par rapport au test de théorie-Comme mentionné plus tôt, le premier but des études d'Analyse Situationnelle était de décrire les forces et les faiblesses des programmes de planification familiale. Plus récemment, les buts de l'étude se sont élargis. Les chercheurs de certains pays ont voulu répondre à un certain nombre de questions à partir d'une étude d'Analyse Situationnelle pour lesquelles elle n'était pas conçue au départ. Il semble exister une divergence d'intérêts entre les chercheurs qui aimeraient utiliser les données pour une analyse des corrélations entre les dynamiques de l'utilisation des services, et les responsables de programmes de planification familiale qui sont plus intéressés par une description de leur programme. Très souvent, les études d'Analyse Situationnelle ont été utilisées pour le dernier de ces deux objectifs plutôt que pour le premier.

- Adaptation pour une gestion routinière et une surveillance du programme-A ce jour, les études d'AS sont organisées et exécutées de façon isolée. Cependant, il y a un intérêt grandissant chez les responsables de programmes SMI et PF dans l'utilisation des procédures de l'assurance de qualité (AQ) pour évaluer et améliorer le fonctionnement de routine de leurs programmes (Katz et al., 1993). Bien que l'approche de l'Analyse Situationnelle dans sa forme actuelle ne soit pas convenable pour être adoptée comme une procédure AQ (Askew, 1994), il est certain de pouvoir l'adapter pour une telle utilisation. Des études au Kenya et au Botswana testent les moyens par lesquels les parties de l'approche de l'Analyse Situationnelle peuvent être utilisées dans les procédures de gestion routinière et de SIG. Dans beaucoup d'autres pays (Kenya, Burkina Faso, Sénégal, Zimbabwe et Ghana) deux études d'Analyse Situationnelle réalisées avec plusieurs années d'intervalle sont en train d'être utilisées pour surveiller les changements du programme dans le temps.

\section{Conclusions}

Depuis la première étude du Kenya en 1989 , l'approche de l'Analyse Situationnelle pour évaluer l'offre de services des programmes de planification familiale s'est diffusée rapidement à travers le monde. L'approche est devenue l'un des principaux outils de diagnostic des forces et des faiblesses du fonctionnement des programmes, d'opérationalisation et d'évaluation du concept de la qualité des soins. L'approche suscite aussi bęaucoup d'espoir en tant que méthodologie pour évaluer l'impact de la qualité des soins sur l'utilisation des services en matière de fécondité des clientes. Les études réalisées à ce jour ont été largement utilisées par les responsables de programmes comme source d'information pour améliorer et étendre les programmes. Comme toute nouvelle approche, la méthodologie a évolué, s'est développée et s'est améliorée. Les tailles des échantillons ont augmenté, de nouveaux sujets comme l'avortement, les MST et les soins 
obstétricaux d'urgence sont en train d'être couverts, et des instruments standard aussi bien que des plans d'analyse de données sont en train d'être utilisés. Dans le même temps, on accorde de plus en plus une attention à plusieurs problèmes d'ordre méthodologique qui n'avaient pas été prévus initialement. Des mesures sont prises pour améliorer la collecte des données. On espère que la méthodologie renforcée, tout en s'appuyant à la fois sur un modèle théorique solide et des méthodes de recherche testées sur le terrain donnera une approche globalement acceptable pour l'évaluation des programmes de planification familiale et de santé de la reproduction.

\section{Références}

Arends-Kuenning, M., B. Mensch, et M. R. Garate. 1996. "Comparing the Peru service availability module and situation analysis." Studies in Family Planning 27,1:44-51.

Askew, I. 1994. "Distinguishing between quality assurance mechanisms and quality assessment techniques." Health Policy and Planning 9,3:274-277.

Askew, I. et A. Fisher. 1995. “Using operations research to guide family planning program development and policy formulation in Sub-Saharan Africa." Population Research and Policy Review 14:373-393.

Bertrand, J., R. Magnani, et J. Knowles. 1994. Handbook of Indicators for Family Planning Program Evaluation. The Evaluation Project, Carolina Population Center, University of North Carolina, Chapel Hill, North Carolina.

Bruce, J. 1990. "Fundamental elements of the quality of care: A simple framework." Studies in Family Planning 21,2:61-91.

Casterline, J., ed. 1985. The Collection and Analysis of Community Data: WFS Seminar on Collection and Analysis of Data on Community and Institutional Factors. 20-23 June 1983, International Statistical Institute, World Fertility Survey.

Center for Human Services. 1988. Primary Health Care Thesaurus: A List of Service and Support Indicators. Chevy Chase, Maryland.

Center for Population and Family Health, Prevention of Maternal Mortality Network. 1995. "Situation Analyses of emergency obstetric care: Examples from eleven operations research projects in West Africa." Social Science and Medicine 40,5:657-667.

Cleland, J. 1973. "A Critique of KAP studies and some suggestions for their improvement." Studies in Family Planning 4,2:42-47.

Dean, A., J. Dean, A. Burton, et R. Dicker. 1990. Epi Info, Version 5: A Word Processing, Database and Statistics Program for Epidemiology on Microcomputers. USD Inc., Stone Mountain.

Diop, N., A. Cerulli, et D. Sanogo. 1996. "Regional dissemination of Senegal's Situation Analysis results: A promising way to maximize operations research utilization." Paper presented at Situation Analysis Managers' Utilization Meeting, Nairobi, Kenya.

Fisher, A., J. Laing, J. Stoeckel, et J. Townsend. 1991. Handbook for Family Planning Operations Research Design, Second Edition. Population Council, New York.

Fisher, A., B. Mensch, R. Miller, I. Askew, A. Jain, C. Ndeti, L. Ndhlovu, et P. Tapsoba. 1992. Guidelines and Instruments for a Family Planning Situation Analysis Study. Population Council, New York.

Fisher, A. 1993. "Family planning in Africa: A Summary of recent results from operations research studies." Dans Conference Proceedings and Papers, Africa OR/TA Project, Africa OR/TA Project, End of Project Conference, Nairobi, Kenya.

Freedman, R. 1974. "Community-level data in fertility surveys." World Fertility Survey Occasional Papers No. 8, Londres, World Fertility Survey.

Frerichs, R. 1989. "Simple analytic procedures for rapid microcomputer-assisted cluster surveys in developing countries." Public Health Reports 104,1:24-34.

Frerichs, R. et Khin Tar Tar. 1989. "Computer-assisted rapid surveys in developing countries." Public Health Reports 104, 1:14-23.

Huntington, D., B. Mensch, et N. Toubia. 1993a. "A new approach to eliciting information about induced abortion." Studies in Family Planning 24,2:120-124.

Huntington, D. et S. Schuler. 1993b. "The simulated client method: Evaluating client-provider interactions in family planning clinics." Studies in Family Planning 24,3:187-193.

Huntington, D., K. Miller, et B. Mensch. 1996a. "The reliability of the situation analysis observation guide." Studies in Family Planning 27,5:277-282.

Huntington, D., B. Mensch, et V. Miller. 1996b. "Survey questions for the measurement of induced abortion." Studies in Family Planning 27,3:155-161.

Katz, K., K. Hardee, et M. Villinski. 1993. Quality of Care in Family Planning: A Catalog of Assessment and Improvement Tools. Family Health International, Durham, North Carolina.

Keller, A. 1991. "Management information systems in maternal and child health/family planning programs: A multi-country analysis." Studies in Family Planning 22,1:19-30.

Kumar, S., A. Jain, et J. Bruce. 1989. "Assessing the quality of family planning services in developing countries." Programs Division Working Paper No. 2, Population Council, New York.

Mensch, B., A. Fisher, I. Askew, et A. Ajayi. 1994a. "Using situation analysis data to assess the functioning of family planning clinics in Nigeria, Tanzania, and Zimbabwe." Studies in Family Planning 25,1:18-31.

Mensch, B., R. Miller, A. Fisher, J. Mwita, N. Keyonzo, F. Mohamed Ali, et C. Ndeti. 1994b. "Family planning services in Nairobi: A Situation Analysis of City Commission clinics." International Family Planning Perspectives 20,2:48-54.

Mensch, B., M. Arends-Kuenning, et A. Jain. 1996. "The impact of the quality of family planning services on 
contraceptive use in Peru." Studies in Family Planning 27,2:59-75.

Miller, R., L. Ndhlovu, et M. Gachara. 1989. A Situation Analysis of the Family Planning Program of Kenya: The Availability Functioning and Quality of MOH Services. Population Council and Kenya Ministry of Health, Nairobi, Kenya.

Miller, R., L. Ndhlovu, M. Gachara, et A. Fisher. 1991. "The situation analysis study of the family planning program in Kenya." Studies in Family Planning 22,3:131-143.

Miller, R., et R. Frerichs. 1992-93. “An integrated approach to operations research for strengthening family planning programs: A case example in Kenya." International Quarterly of Community Health Education 13,3:183-199.

Miller, R., K. Miller, L. Ndhlovu, J. Solo, et O. Achola. 1996. "A comparison of the 1995 and 1989 Kenya Situation Analysis study findings." Document présenté à la réunion Kenya: Situation Analysis Study of the Family Planning Services. Population Council and Division of Family Health, Ministry of Health, Naïrobi. Autti presenté à la réunion annuelle de l'APHA, Novembre 1996, New York.
Population Council, Africa Operations Research et Technical Assistance Project II. 1996. Case Study Summary Report: A Situation Analysis of the National Family Planning Program in Burkina Faso. Population Council, Dakar, Senegal.

Ross, J. et E. Frankenberg. 1993. Findings from Two Decades of Family Planning Research. Population Council, New York.

Sloan, N., C. Quimby, B. Winikoff, et N. Schwalbe. 1995. Guidelines and Instruments for a Situation Analysis of Obstetric Services. The Ebert Program du Population Council, New York.

Simmons, R. et C. Elias. 1993. "The study of client-provider interactions: A review of methodological issues." Programs Division Working Paper No. 7, Population Council, New York.

Wilkinson, M. 1991. "The DHS service availability questionnaire." Paper presented at the meeting on Measuring the Influence of Accessibility of Family Planning Services in Developing Countries, National Academy of Sciences Committee on Population, Washington, DC. 


\section{Exécution de l'étude}

\section{Problèmes d'ordre éthique dans l'exécution des études d'AS}

Les études d'AS nécessitent des interviews auprès des client(e)s et des prestataires, aussi bien que des observations des interactions prestataire-cliente. Ces procédures d'études pourraient constituer un risque pour les sujets d'étude à moins que des mécanismes ne soient établis pour les protéger. Les institutions qui s'apprêtent à réaliser des études d'Analyse Situationnelle ou des activités d'assistance technique locale devraient tenir compte des lois en vigueur ainsi que des dispositions qui régissent la révision des protocoles et des procédures de recherche. Dans plusieurs pays, des comités spéciaux révisent les protocoles de recherche pour s'assurer que les questions de confidentialité, d'intimité, de sensibilité, de consentement éclairé et d'autres préoccupations générales seront traitées de manière éthique afin de protéger les sujets de l'étude de certains torts.

The Council for International Organization of the Medical Sciences (CIOMS), affilié à l'Organisation mondiale de la Santé, réfère les questions d'ordre éthique préoccupantes à la communauté internationale de recherche. Les quatre directives du CIOMS en matière de recherche sur les sujets humains sont':

“1. Le respect des personnes, ce qui inclut à la fois l'autonomie, ou le respect de l'autodétermination de ceux qui peuvent délibérer sur leurs buts personnels, et la protection des personnes ayant une autonomie limitée ou diminuée;

2. la bienfaisance, qui est l'obligation éthique de maximiser les profits et de minimiser les dommages ou les torts;
3. la non malfaisance, qui veut dire "ne pas faire de mal" ; et

4. la justice, qui exige que les sujets de l'étude soient traités équitablement, et que les études soient conçues de manière à ce que les sujets de l'étude soient aussi les bénéficiaires de l'étude...."

Toutes ces préoccupations sont pertinentes dans une étude d'Analyse Situationnelle et devraient être discutées ouvertement avec les chercheurs et les responsables de programmes au niveau de la localité, et avec le personnel de terrain pendant la formation. Toute l'importance de l'intimité, du consentement éclairé et de la protection des sujets doit être communiquée à tous ceux qui sont impliqués dans l'étude. Aussi, les chercheurs ont-ils besoin d'apprendre à communiquer ces messages aux sujets de l'étude et à toutes autres personnes concernées.

Confidentialité et intimité. L'information obtenue des prestataires et des clientes dans le cadre d'une étude d'Analyse Situationnelle doit être gardée de manière confidentielle et privée, suivant la directive de non malfaisance. L'identité des sujets de l'étude doit être protégée par les chercheurs, ce qui est fait d'habitude en identifiant les sujets à l'aide d'un code plutôt qu'avec un nom ou une adresse. De même, les chercheurs devraient faire attention pour contrôler ou supprimer toutes variables qui pourraient être utilisées pour identifier individuellement les client(e)s interviewé(e)s.

\footnotetext{
' De T.L. Beauchamp et J.L. Childress, Principles of Biomedical Ethics, 2nd Edition, Oxford University Press, 1983, comme résumé dans K. Ringheim, 1995, "Ethical issues in social science research with special reference to sexual behaviour research", Social Science and Medicine, 40,12:1691-1697.
} 
Dans certains pays, le cadre conceptuel de l'étude d'Analyse Situationnelle exige un groupe de clientes qui seront suivies dans le temps et interviewées à nouveau. Dans ce type de cadre conceptuel, on doit demander aux clientes à la fin de l'interview si elles désirent faire partie du groupe, si elles veulent que leurs noms et adresses soient enregistrés, et si elles souhaitent être interviewées plus tard à un moment donné. A ce niveau, les sujets de l'étude doivent être informés du fait que des contacts de suivi peuvent avoir lieu à leurs domiciles, où un conjoint ou un membre de la famille peut apprendre que la cliente interviewée reçoit des services cliniques tels que la planification familiale ou un traitement pour une MST. Si la cliente ne veut pas faire partie de l'étude, alors les chercheurs devraient respecter sa décision. Son nom et son adresse ne peuvent être enregistrés qu'après son consentement. Cependant, puisque cette information identifie directement les clientes, les noms et les adresses doivent être enlevés et gardés séparément dans une armoire fermée dont l'accès est limité. La seule identification sur le questionnaire devrait être un numéro de code.

La confidentialité s'étend non seulement à l'identité de la cliente mais aussi à l'exposition dont elle peut faire l'objet pendant toute l'étude. L'observation et l'interview de fin de consultation traitent toutes les deux de questions très sensibles de la vie familiale et de comportement sexuel, ce qui pourrait mettre la cliente mal à l'aise, surtout si d'autres personnes peuvent écouter les réponses que la cliente donne aux questions. Il est très important de faire les interviews dans un endroit privé où la cliente peut être à l'aise (ceci augmente aussi la validité de l'information collectée). De même les examens physiques sont souvent indiscrèts et gênants pour les clientes. Les observateurs des études d'Analyse Situationnelle devraient toujours obtenir le consentement de la cliente pour être présents pendant l'examen. Le désir d'intimité de la cliente dans ces lieux doit être respecté.

Consentement éclairé. Le principe du respect des personnes établit le droit des clientes et des prestataires d'être informés sur la nature de l'étude, sur tous les risques et avantages liés à leur participation, et qu'ils peuvent se retirer de l'étude à n'importe quel moment. Les clientes doivent être informées aussi qu'elles peuvent recevoir tous les services du centre de santé indépendamment de leur participation ou non à l'étude. Ces questions devraient être présentées dans une langue facilement compréhensible, et le sujet doit consentir librement et volontairement à son inclusion dans l'étude. Le vrai "consentement éclairé" nécessite aussi qu'on donne au sujet l'opportunité de poser des questions sur l'étude avant de donner son consentement. Le personnel de terrain devrait être informé de la manière de traiter et de diriger les questions posées par les sujets de l'étude sur la planification familiale et autres problèmes liés à la santé. Des directives claires doivent être données au personnel de terrain pour souligner la nature purement volontaire de toute participation. Il importe aussi de noter que le consentement ne devrait être obtenu que lorsque la cliente n'est pas sous contrainte, ne souffre pas, n'est pas traitée avec des médicaments qui agissent sur sa lucidité, ne sollicite pas de soins intensifs. Les clientes qui présentent l'une de ces conditions ne devraient pas être incluses dans l'étude puisque leur condition les empêche de réfléchir attentivement sur leur consentement.

Les avantages que les clientes et les prestataires tireront de l'étude sont surtout d'ordre social : l'information sera utilisée pour développer et améliorer les politiques et les pratiques des programmes. Nous pensons que les procédures de l'Analyse Situationnelle n'entraînent pas de risques supplémentaires en dehors des risques sociaux et psychologiques que peuvent provoquer la recherche d'une consultation pour un problème lié à la sexualité. Bien qu'il soit possible qu'une cliente coure un risque supplémentaire de la part d'un prestataire fâché du fait qu'on l'observe, nous croyons que la probabilité la plus importante est que le prestataire devienne particulièrement attentif pendant l'observation et essaye de fournir des services de la plus haute qualité. 
Responsabilité de l'observateur pour le bien-être de la cliente. La Déclaration d'Helsinki en 1964, qui porte sur l'éthique en matière de recherche biomédicale, stipule "qu'en matière de recherche sur l'être humain, l'intérêt de la science et de la société ne devrait jamais l'emporter sur les considérations liées au bien-être du sujet." Parfois, les observateurs de l'Analyse Situationnelle constatent des violations flagrantes du comportement acceptable des prestataires qui mettent la cliente dans un risque de morbidité. Par exemple, si un prestataire laisse tomber un DIU par terre, le ramasse et tente de l'insérer à une cliente sans le stériliser, il expose la cliente à un risque d'infection. Dans un cas, on a observé un prestataire essuyer ses mains sur les rideaux avant de faire un examen physique. Dans de tels cas de violation flagrante de procédures standards acceptables, l'observateur de l'étude d'Analyse Situationnelle peut prendre la responsabilité d'intervenir pour protéger la cliente d'un dommage potentiel. Une liste de situations potentielles qui nécessitent une forme d'intervention devrait être développée et discutée pendant la formation par les cadres du Ministère de la santé.

Il est vrai que les interventions de ce type peuvent influer sur les résultats de la recherche, mais dans de telles circonstances la sécurité de la cliente l'emporte sur les besoins des chercheurs et des responsables de programmes. Lorsque les intérêts des clientes et ceux des chercheurs sont clairement opposés, les besoins de la recherche devraient être compromis comme le recommande la Déclaration d'Helsinki. Ces cas devraient aussi être bien documentés. Les observateurs de terrain sont formés en conséquence dans les études d'Analyse Situationnelle assistées par le Population Council.

Dans les cas moins extrêmes, les observateurs peuvent se trouver confrontés à un problème d'éthique plus difficile et moins évident. Il est probable que beaucoup de chercheurs s'accordent sur la nécessité de l'intervention de l'observateur dans les cas extrêmes où il est claire que la cliente court un risque. Cependant, ces cas sont relativement rares. Les faits les plus couramment trouvés dans presque toutes les études d'Analyse Situationnelle c'est une grande diversité de plus de qualité de services moins sérieux. En effet, c'est généralement le cas, les observateurs verront beaucoup d'erreurs, de défaillances et de désinformation transmise, et les enquêteurs trouveront que les clientes peuvent ne pas connaître les informations capitales sur la méthode contraceptive choisie. En effet, l'Analyse Situationnelle est conçue juste pour découvrir ce genre d'information pour que les changements nécessaires de politique et de programme puissent être faits. Devrait-on tenter une intervention avec chaque situation de prestation de services qui est moins optimale ? Il est évident que cette décision s'avérerait non seulement gênant et nuisible à réaliser avec le personnel, mais aussi détruirait la possibilité de rassembler une information utile pour les prises de décision relatives aux programmes et à la politique, et de ce fait amoindrirait la capacité de faire des améliorations nécessaires. Il est important de bien discuter avec les autorités locales compétentes des éventuels problèmes éthiques qui peuvent se poser pendant l'exécution d'une étude d'Analyse Situationnelle, et les types de comportements des prestataires qui peuvent nécessiter une forme d'intervention de la part des observateurs et des enquêteurs de l'étude.

\section{Etablissement des objectifs et révision des questionnaires}

La première étape de la réalisation d'une étude d'Analyse Situationnelle est de tenir une réunion de planification ou d'orientation pour les chercheurs, les principaux responsables de PPS à visiter et les principaux utilisateurs de données (tels que les décideurs et les bailleurs de fonds). Pendant cette réunion, on explique en détails la méthodologie et les procédures de l'AS, et on discute des objectifs de l'étude. Après que ces points aient été tirés au clair, les questionnaires sont révisés attentivement pour voir s'ils sont justes. Les objectifs de l'étude devraient proposer au groupe le type d'information 
TABLEAU 1. Echantillon du programme de formation

\begin{tabular}{|c|c|}
\hline Jour & Activités \\
\hline \multirow[t]{6}{*}{ Un } & Introductions. \\
\hline & Plan de formation. \\
\hline & Contexte des études d'AS. \\
\hline & Concepts de fiabilité et quelques pièges dans le processus de collecte des données. \\
\hline & Quelques expériences de terrain d'autres études d'AS \\
\hline & Vue d'ensemble sur les instruments. \\
\hline \multirow[t]{3}{*}{ Deux } & Révision du guide d'observation. \\
\hline & Révision de l'interview de fin de consultation de la cliente PF. \\
\hline & Révision de l'interview du personnel. \\
\hline \multirow[t]{4}{*}{ Trois } & Révision de l'inventaire. \\
\hline & Révision de l'interview de fin de consultation de la cliente SMI. \\
\hline & Réunion plénière pour discuter des questions qui se posent lors de la révision des instruments. \\
\hline & Directives pour faire les interviews. \\
\hline \multirow[t]{3}{*}{ Quatre } & Répartition des enquêteurs et des infirmières/sages-femmes en groupes spécialisés. \\
\hline & Révision de l'administration des instruments spécifiques pour chaque groupe. \\
\hline & Jeux de rôle. \\
\hline \multirow[t]{2}{*}{ Cinq } & Suite des jeux de rôle. \\
\hline & Réunion plénière pour discuter les expériences acquises des jeux de rôle. \\
\hline \multirow[t]{2}{*}{ Six } & Travail de terrain dans des PPS sélectionnés. \\
\hline & Pratique de l'administration des instruments de collecte des données par chaque équipe. \\
\hline Sept & Suite du travail de terrain dans des PPS sélectionnés. \\
\hline \multirow[t]{2}{*}{ Huit } & Révision des expériences acquises dans le travail sur le terrain et dans les jeux de rôle. \\
\hline & Révision des rôles de supervision avec les Chefs d'équipe/Superviseurs. \\
\hline Neuf & Jeux de rôle. \\
\hline \multirow[t]{3}{*}{ Dix } & Chaque équipe planifie sa logistique de voyage. \\
\hline & Plans discutés en groupe et modifiés si nécessaire. \\
\hline & Questions d'ordre administratif et financier. \\
\hline
\end{tabular}

qui sera nécessaire, et un groupe d'indicateurs clés peuvent être généralement fixés à ce niveau (voir chapitre 4 pour les indicateurs proposés). Ces indicateurs devraient guider toutes les révisions apportées aux questionnaires.

En outre, le plan d'échantillonnage devrait être développé pour refléter les besoins de toutes les organisations de planification familiale concernées du pays, et les responsables des programmes devraient se mettre d'accord sur les responsabilités afin d'assurer l'accès de l'équipe de recherche aux PPS. En plus, on devrait continuer à impliquer les responsables de programmes dans toutes les activités de l'étude y compris les phases de formation et de collecte des données sur le terrain.

\section{Programme de formation de l'équipe de recherche}

L'étape suivante est d'organiser une formation pour l'équipe de recherche, ce qui nécessite généralement dix jours. La formation consiste en une introduction générale aux objectifs de la recherche, aux interviews et aux observations, aux questions d'ordre éthique, et en une révision détaillée de chaque question dans chaque questionnaire afin que tous les membres de l'équipe comprennent le sens de chaque question, comment poser la question et comment enregistrer la réponse. Un temps considérable est ensuite consacré "au jeu de rôle" des interviews et des observations, avec les enquêteurs jouant tour à tour 
le rôle de l'interviewer ou de l'observateur, et celui du personnel de PPS ou de la cliente. Le jeu de rôle donne à chaque membre de l'équipe la chance de s'entraîner à poser des questions et à enregistrer les réponses. Au départ, il est très fréquent que les enquêteurs enregistrent les réponses différemment. La discussion de ces différences donne l'opportunité de clarifier les définitions et les compréhensions. Au fur et à mesure que le programme de formation progresse, les enquêteurs développeront une confiance avec les instruments de collecte des données et mémoriseront probablement certaines sections. La confiance chez les observateurs augmente. En plus du jeu de rôle, la session de formation inclut au moins un séjour de deux jours dans les PPS afin que les membres de l'équipe puissent réellement s'entraîner à interviewer, à observer et à faire l'inventaire. Voir les activités de l'échantillon du programme de formation ébauché dans le tableau 1.

Le dernier jour de la formation est consacré à la planification du travail sur le terrain. Chaque équipe se réunit pour mettre au point les activités de terrain suivantes:

- la liste des PPS à visiter;

- le calendrier des visites pendant la période de travail sur le terrain;

- le transport;

- l'hébergement;

- les visites de supervision;

- le stockage des instruments de collecte des données qui sont remplis et le réapprovisionnement en nouveaux instruments.

Le travail sur le terrain devrait commencer une semaine après cette dernière session, ce qui donnera le temps aux chercheurs de l'étude de réviser et d'imprimer les nouveaux questionnaires. Quand ceci sera fait et que les instruments seront distribués aux équipes de terrain, le programme de visite dans les PPS pourra commencer.

\section{Rôle du Chef d'équipe/Superviseur}

Le chef d'équipe sera appelé à jouer plusieurs rôles sur le terrain. Il devrait être prêt à apporter son assistance à tout moment en ce qui concerne les opérations sur le terrain. La bonne entente entre les membres de l'équipe est un facteur important à la bonne exécution du travail sur le terrain. Cela veut dire que chaque membre de l'équipe doit être sensible aux besoins et aux points de vue des autres membres. Là où des problèmes se posent, le superviseur peut avoir la responsabilité de faciliter le processus de résolution des problèmes. Avant tout, il faut se dire que chaque membre de l'équipe dispose de ressources et de capacités différentes, et que celles-ci devraient être exploitées pour que l'expérience sur le terrain soit couronnée de succès. Quelques questions auxquelles on doit accorder une attention quand on est sur le terrain sont discutées ci-dessous :

- Arrangement pour le transport-Le chef d'équipe doit s'assurer que les moyens de transport sont disponibles et prêts tous les jours pour amener les enquêteurs sur le terrain. Le chauffeur qui devient membre de l'équipe de terrain doit recevoir des instructions claires sur la destination et l'heure de départ. Le transport en commun peut être utilisé comme alternative si cela s'avère approprié, mais ce moyen de transport n'est généralement pas satisfaisant sauf peut-être dans les zones urbaines.

\section{- Vérification du remplissage des} instruments-Sur une base journalière, le superviseur contrôle les entrées des instruments de collecte des données pour vérifier la consistance et la précision des données collectées. Ceci inclut les enregistrements erronés durant les interviews. Si les révisions sont faites en groupe, cela sert de formation aux membres du groupe. Ce processus est particulièrement important pendant les premiers jours de l'étude. Pour tous les instruments, quelques informations peuvent être portées sur la page de couverture avant d'effectuer la visite dans un PPS. Cette méthode est encouragée puisqu'elle permet de gagner du temps et de 
se planifier d'avance pour être sûr que toutes les informations sont consistantes.

Pour maintenir un approvisionnement suffisant en instruments, le chef d'équipe doit garder un contact permanent avec le chercheur principal au niveau du bureau central. De préférence, un jour sera fixé pendant les sessions de formation pour une visite de terrain du chercheur principal.

- Stockage des questionnaires en lieu sûr-Le chef d'équipe doit organiser le bon stockage des instruments en lieu sûr, ceux qui sont utilisés et ceux qui ne sont pas encore utilisés. Les instruments doivent être gardés dans un ordre logique selon le type et la date de visite des PPS.

- Obtention de la permission des autorités locales-La permission de visiter les PPS doit être obtenue des autorités locales. Il revient au chef d'équipe de s'occuper de cet aspect. Dans les PPS mêmes, les membres de l'équipe devraient être présentés au personnel du PPS et le but de l'étude expliqué brièvement. Généralement, cette fonction revient au chef d'équipe.

- Paiement des per diems et autres questions pécuniaires-Chaque équipe met en place sa propre organisation des repas et autres besoins courants. Généralement, les arrangements des per diems de l'équipe seront faits par le chef d'équipe.

D'habitude, le chef d'équipe garde de l'argent pour le carburant et autres dépenses relatives au véhicule. Il importe d'obtenir et de garder les reçus des dépenses correctement et de façon ordonnée. Un autre point relatif au véhicule est que le chef d'équipe doit vérifier que le chauffeur tient correctement un carnet du kilométrage quotidien.

- Tenue d'un carnet des activités quotidiennes-A la fin de chaque journée, le Chef d'équipe révise en compagnie des autres membres de l'équipe les activités du jour et rédige un petit rapport (généralement environ $1 / 2$ page à 1 page). Le rapport peut inclure les événements qui ont rendu la collecte de données inhabituelle-peut-être trop facile ou trop difficile. Le rapport est un résumé des expériences vécues par l'équipe et constitue une information utile pour l'interprétation des résultats de la recherche.

- Contact permanent avec le bureau centralSi l'équipe rencontre des difficultés inhabituelles ou doute de certaines questions, le bureau central ou le superviseur devrait être contacté immédiatement.

\section{L'équipe de recherche et une journée type de travail}

Comme mentionné dans le chapitre précédent, le nombre d'équipes de recherche et de superviseurs utilisés dans une étude dépend du nombre et de l'accessibilité des PPS, des objectifs choisis et des ressources disponibles. D'habitude entre 5 et 10 équipes séjourneront environ pendant six semaines pour visiter un total de 150 à 300 PPS; chaque équipe visitera 25-30 PPS.

Avant de se rendre dans un PPS, peut-être la veille, les membres de l'équipe devraient s'assurer qu'ils sont d'accord sur les "codes standard" pour identifier le type et la localisation du PPS à visiter, et si possible les écrire d'avance sur la page de couverture et la page de garde de chaque instrument à utiliser. Les codes pour le type de PPS, le type de secteur et le type de la localité devraient être discutés avec le Chef du PPS et devraient être consistants pour tous les instruments utilisés dans ce PPS. En outre, les codes pour chaque membre du personnel et chaque cliente doivent être enregistrés attentivement pour chaque interview et chaque observation, et doivent correspondre dans tous les instruments. Le Chef d'équipe devrait attribuer ces codes aux membres du personnel et aux clientes après l'arrivée de l'équipe dans le PPS. Ces variables de "liaison" sont extrêmement importantes dans l'analyse des données. De ce fait, ces codes devraient être vérifiés attentivement chaque fois par le Chef d'équipe. Voir chapitre 3 pour plus d'information. 
L'équipe devrait toujours arriver dans le PPS avant l'heure d'ouverture officielle. Ceci est nécessaire pour leur permettre d'observer ce qui se passe quand le PPS s'ouvre et, bien sûr, s'il s'ouvre à l'heure.

Comme mentionné dans le chapitre précédent, le membre de l'équipe qui a une formation clinique commence d'habitude à observer l'interaction entre les clientes de planification familiale et le(s) prestataire(s) au début de la journée. Plus tard, cette même personne aidera un autre membre de l'équipe à faire l'inventaire de l'équipement. Le spécialiste en sciences sociales est généralement chargé des interviews de fin de consultation des clientes et des interviews du personnel. Il/elle peut aussi aider dans la collecte des informations sur les statistiques de la clinique, sur les rapports et sur les statistiques des services. Ces deux membres de l'équipe sont chargés de l'interview des clientes SMI.

A la fin de la journée, le Chef d'équipe devrait réviser en compagnie des autres membres de l'équipe les activités de visite dans le PPS. Ensuite le Chef d'équipe devrait rédiger un bref rapport ( $1 / 2$ page à 1 page) sur la visite, tout en relevant toutes les circonstances ou événements inhabituels qui sont importants mais ne seront pas apparents à partir des instruments de collecte des données.

En plus, le Chef d'équipe doit réviser aussi chaque instrument pour s'assurer que toutes les réponses sont correctement enregistrées. Cette étape est souvent difficile à réaliser à la fin d'une longue journée, mais on ne pourra jamais assez souligner son importance. Si une information manque dans les questionnaires, la qualité des données est fortement compromise, et les résultats seront moins fiables et même quelquefois inutilisables. Le coût des données manquantes est donc très élevé et devrait être évité par tous les moyens. La vérification pour identifier les données manquantes à la fin de la journée sur le terrain peut être harassante, mais elle représente la dernière opportunité de compléter toute information manquante.
Astuces pour réaliser une bonne interview L'objectif de toute étude d'Analyse Situationnelle est de collecter l'information la plus précise possible. Collecter l'information par le biais d'interviews n'est pas toujours facile et nécessite une habileté à poser clairement les questions et à écouter les réponses. L'objectif d'une interview est d'avoir les opinions et les idées de la personne interviewée. Il est très important d'être impartial lorsqu'on mène une interview auprès d'une personne. Evitez de mentionner que la réponse est correcte ou non. Les seules réponses correctes sont celles données par la personne interviewée. Voici quelques astuces pour réaliser une bonne interview.

- Présentez-vous toujours de façon amicale et plaisante.

- Soyez sûrs de mettre l'accent sur la nature volontaire et confidentielle de l'interview. Si une personne refuse d'être interviewée, mettez fin à l'interview de manière polie et passez à une autre personne.

- Ne changez jamais la formulation des questions. Posez chaque question exactement de la même manière qu'elle est écrite dans le questionnaire. Utilisez un ton impartial.

N'essayez pas d'orienter le/la répondant(e) vers une réponse ou une autre. Ne pas souffler les réponses au répondant. Laissez-le répondre de lui-même.

- Si vous ne comprenez pas la réponse à une question, demandez au répondant de répéter la réponse. Mais, il ne faut pas "guider" le répondant comme si vous lui proposiez une réponse. De manière impartiale, demandez au répondant: "Pouvez-vous expliquer davantage? Prenez votre temps, il n'y a pas lieu de se presser."

- Si le répondant ne comprend pas une question, répétez-la lentement et clairement. Si le répondant ne comprend toujours pas la question, vous pouvez la reprendre en utilisant d'autres mots-mais faites très attention à ne pas changer le sens de la question.

- Ne bousculez pas le répondant ou n'agissez pas comme si vous étiez pressés. Donnez au 
répondant assez de temps possible pour répondre à vos questions.

- Encerclez le numéro de la question, cochez l'encadré ou écrire le numéro qui correspond à la réponse donnée par le répondant. Encerclez ou cochez une seule réponse à moins que la question n'indique clairement que plus d'une réponse peut être donnée, auquel cas encerclez ou cochez les catégories concernées.

- S'il y a des difficultés avec une question particulière ou si quelque chose d'inhabituel se produit comme le départ soudain du répondant, écrivez ce qui s'est passé à la marge du questionnaire ou au verso. A la fin de la journée, expliquez au Chef d'équipe ce qui s'est passé.

- Souvent on vous demandera d'écrire une réponse dans la partie "Autre : __ du questionnaire. Ecrivez clairement et simplement. Utilisez le verso du questionnaire si vous avez besoin davantage d'espace, mais rassurez-vous que vous avez identifié le numéro de la question à laquelle votre réponse s'applique.

\section{Astuces pour réaliser une bonne observation} L'observation des interactions cliente-prestataire fournit la plupart des informations sur la manière dont la cliente a été conseillée, examinée et la façon dont on lui a fourni une méthode contraceptive. La personne qui fait l'observation doit toujours avoir une formation médicale et une grande expérience dans la prestation des services de planification familiale. Les observateurs les mieux indiqués sont normalement des infirmières/sages-femmes qui ont plusieurs années d'expérience en matière de planification familiale et qui sont aussi formatrices en planification familiale. Leur expérience en matière de formation leur permet de percevoir l'observation sous un angle critique, et tous les comportements de prestataires énumérés dans l'instrument d'observation leur seront familiers.

Le guide d'observation est conçu de sorte que l'observateur coche les encadrés ou encercle les numéros qui décrivent ce qu'il a vu. Puisqu'il n'y a pas un ordre fixe pour chaque consultation, il est essentiel que l'observateur apprenne la structure du guide d'observation de sorte que, chaque fois qu'il voit une action particulière ou entend discuter d'une question spécifique, il sache exactement où noter dans le guide. Dans certains cas, il peut être nécessaire de se rappeler de ce qui s'est passé et de noter dans le guide après la consultation.

Avant la première consultation, l'observateur doit avoir la permission du prestataire pour s'asseoir et observer l'interaction entre la cliente et le prestataire. Normalement, cela ne constitue pas un problème, mais il est important de rassurer le prestataire que vous ne faites pas une évaluation de sa compétence. Il peut y avoir des problèmes si le prestataire connaît l'observateur soit comme superviseur ou comme collègue. Donc, tout doit être mis en oeuvre pour éviter que cela ne se produise.

On devrait aussi informer les prestataires que l'observateur de la recherche ne peut pas intervenir du tout pendant les consultations. Le prestataire ne devrait pas demander les points de vue de l'observateur ou solliciter des conseils auprès de lui, sauf dans les situations extrêmement graves. On demandera au prestataire de se comporter comme si l'observateur n'était pas présent.

L'observateur devra s'asseoir assez proche de la cliente et du prestataire afin de pouvoir voir et entendre ce qui se passe. En plus sa présence va sans doute influer sur l'interaction, peut-être en incitant la cliente et le prestataire à devenir plus timides et à prendre conscience de ce qu'ils disent et ce qu'ils font. Par conséquent, l'observateur devrait faire tout ce qui est possible pour être effacé.

Avant de commencer la consultation, le prestataire devrait demander à la cliente si elle est d'accord pour que l'observateur soit présent. Si possible, l'observateur devrait s'installer à l'arrière plan de sorte qu'il ne se trouve pas face à face avec le prestataire ou la cliente. Il devrait s'habiller 
correctement (dans certains cas les observateurs portent leurs propres blouses) et avoir le sourire aux lèvres. Il devrait aussi garder ses papiers et son crayon sur ses cuisses et être discret lorsqu'il prend note d'une observation.

Rarement, l'observateur peut voir un prestataire en train de faire quelque chose qui peut être dangereux pour la cliente. A titre d'exemple, le prestataire peut être en train d'utiliser un équipement non stérilisé pour faire un examen, poser un DIU ou faire une injection. Pendant la formation, une liste des situations que le Ministère de la Santé pense être en violation flagrante des pratiques sûres sera établie. Et lorsque ces situations sont constatées par l'observateur, elles devraient être arrêtées. (Voir la section précédente sur les problèmes d'ordre éthique, page 16.)

A la fin de la consultation, l'observateur devrait raccompagner la cliente et lui demander si elle ne voit pas d'inconvénient à être interviewée. Si elle accepte, alors l'observateur devrait la présenter à l'enquêteur spécialiste des sciences sociales et lui remettre le guide d'observation / instrument d'interview de fin de consultation. Il est très important que l'instrument utilisé pour l'observation de l'interaction cliente-prestataire soit remis à l'enquêteur spécialiste des sciences sociales (voir chapitre 3).

\section{Après la collecte des données}

Généralement, la collecte des données peut être réalisée dans quatre ou six semaines pour une étude d'Analyse Situationnelle au niveau national. A la fin de la collecte des données, il est très important de faire un compte rendu oral avec les superviseurs et les enquêteurs. Le chercheur principal, les principaux responsables de programmes ainsi que les administrateurs devraient se réunir avec les équipes de recherche et réviser l'ensemble du processus de collecte des données. Toutes circonstances inhabituelles devraient être notées.

Voir le chapitre 4 pour une information sur la saisie, le nettoyage, l'analyse des données ainsi que la rédaction des rapports. 


\section{Instruments et guides question par question}

Ce chapitre comprend les instruments de l'Analyse Situationnelle qui sont :

- Inventaire des installations disponibles et des services fournis dans le PPS

- Guide d'observation de l'interaction entre les clientes de planification familiale et les prestataires de services

- Interview de sortie des clientes de planification familiale

- Interview du personnel prestataire des services de planification familiale/santé de la reproduction dans le PPS

- Interview des clientes SMI fréquentant le PPS. Chaque question sur les instruments est marquée par un nom de variable proposée entre accolades : \{\}. Ces noms de variables n'apparaitront pas dans les questionnaires utilisés sur le terrain, mais elles seront utilisées pendant la saisie et l'analyse des données. Les noms de variables sont donnés à partir des numéros des questions (et non pas à partir de leur contenu) parce que cette méthode de nomination s'est révélée la plus facile à utiliser pendant l'analyse des données. Pour plus d'information sur les noms des variables, voir la section sur la saisie et le nettoyage des données (page 161).

Chaque instrument est suivi d'un guide question par question qui explique le but et les détails de chaque question. Ces guides s'adressent à l'agent de collecte des données et devraient être lus en même temps que les instruments pendant la formation puisqu'ils fournissent l'information essentielle sur la manière d'administrer chaque question.

Plusieurs questions sont communes à tous les instruments et ne sont pas discutées directement dans les guides question par question. Ces questions apparaissent sur les pages de couverture de chaque instrument, et sont particulièrement importantes dans l'analyse des données. Elles incluent :

- le code de la structure sanitaire

- le code du district

- le code du village/de la ville

- le type de structure sanitaire

- le type de secteur

- la localité

- le Numéro d'étude de la cliente

- le Numéro d'étude du personnel

Les codes de la structure sanitaire, du district et du village/ville devraient être fixés de commun accord avant de commencer le travail sur le terrain. Si possible, ces listes devraient être copiées et envoyées en même temps que les équipes de recherche pour référence pendant la collecte des données. L'information relative au type de structure sanitaire, au secteur et à la localité peut être recueillie dans le PPS, mais ces réponses devraient correspondre dans les inventaires, les observations, les interviews du personnels et les interviews de fin de sortie qui sont réalisées dans le même PPS.

Le Numéro d'étude de la cliente et celui du personnel nécessitent une coordination de la part du Chef d'équipe. Ces numéros ne servent qu'à identifier le personnel et les clientes dans un PPS donné. Par exemple, le premier PPS visité peut avoir trois prestataires de services qui travaillent ce jour-là. Ces prestataires devraient être numérotés de 1 à 3 au début de la journée. Si le premier prestataire est interviewé, le Chiffre " 1 " serait marqué sur la page de couverture de la fiche d'interview du personnel. Et si le prestataire est observé avec une cliente, le même Chiffre serait 
marqué sur la page de couverture de la fiche d'observation. De même, si ce prestataire est observé avec quatre clientes, alors ces clientes seraient numérotées de 1 à 4 , et ce même Chiffre serait marqué sur les pages de couverture des fiches d'observation et d'interview de sortie de la cliente. Si le personnel Numéro "2" est observé avec trois clientes, elles devraient aussi être numérotées de 1 à 3 , etc. De cette manière, une liaison peut être établie entre le personnel et les clientes par le biais des observations. Quand on visite le prochain PPS, les numéros d'identification du personnel peuvent commencer encore à partir de 1. Puisque ce procédé peut porter à confusion, seul le Chef d'équipe devrait attribuer les numéros d'étude du personnel et des clientes, et vérifier attentivement à la fin de la journée que ces numéros sont codés correctement.

Souvent, les clientes et les prestataires se font des soucis du fait qu'ils sont identifiés individuellement dans les questionnaires et lors de l'observation. Pourtant, ces numéros d'étude ne sont utilisés que pour lier l'information entre les instruments, et ne sont jamais utilisés pour identifier les individus. Les noms des clientes et du personnel ne sont jamais relevés, et aucune autre identification d'individus n'est demandée. 


\section{Inventaire}

Le but de cet instrument est d'évaluer la capacité d'un PPS à fournir des services. Il fait le décompte des services disponibles, établit une liste complète de tout l'équipement, des provisions, des matériels et des produits en bon état. Il évalue aussi le fonctionnement de plusieurs composantes y compris l'infrastructure physique, la dotation en personnel, les matériels d'IEC, la logistique, la gestion, la supervision et le stockage des données. Le guide question par question de l'inventaire contient une information détaillée sur la manière de compléter chaque question.

Les codes présentés entre accolades \{\} dans cet instrument sont des noms de variables proposés pour chaque question ou marque à cocher. On se réfère à ces noms dans le plan d'analyse (commençant à la page 166). Cependant, il faut garder à l'esprit qu'ils n'apparaitront pas dans le questionnaire utilisé par les chercheurs sur le terrain. Voir les pages qui suivent pour l'instrument. 


\section{Inventaire des installations disponibles et des services offerts dans le Point de Prestation de Services}

INSTRUCTIONS A L'ATTENTION DE L'AGENT DE COLLECTE DE DONNEES : Cet inventaire doit être complété par une observation des installations disponibles et suite aux discussions avec la personne responsable de la SMI/planification familiale le jour de la visite. Dans tous les cas vous devriez vérifier vousmême que les éléments existent effectivement. S'il ne vous est pas possible de les voir vous-même, alors remplissez le questionnaire en conséquence. Gardez à l'esprit qu'il s'agit d'identifier l'équipement et les installations qui existent actuellement et non d'évaluer la prestation du personnel ou de la structure sanitaire. Pour chaque élément, encercler ou décrire la réponse, selon le cas.

Structure sanitaire visitée (nom) :

\{NOMFS\}

Code de la structure sanitaire :

\{CODEFS\}

District (nom) :

$\{$ NOMDIST\}

Code du district :

\{CODEDIST\}

Village/ville (nom) :

\{NOMVILLE\}

Code Village/ville :

\{CODEVILLE\}

Date de la visite : \{DATE\}

Jour

Mois

Année

Type de la structure sanitaire: \{TYPE\}

Type de secteur: $\{$ SECT $\}$

1 = Hôpital de référence

2 = Hôpital de district

1 = Gouvernement

3 = Hôpital primaire

2 = APF

$3=$ Mission

4 = Centre de santé rural

5 = Maternité

6 = Poste de santé

4 = Privé

$5=$ Autre

7 = Pharmacie

$8=\mathrm{DBC}$

9 = Autre

Localité: \{LOC $\}$

1 = Rurale

2 = Urbaine

Nom de l'observateur :

Signature du chef d'équipe : 


\section{ACCESSIBILITÉ}

DEM 1. Quelle est l'heure d'ouverture officielle de cette structure sanitaire ? $\{11\}$ $\mathrm{am} / \mathrm{pm}$

OBS 2. A quelle heure la première cliente de planification familiale est-elle arrivée aujourd'hui ? $\mathrm{am} / \mathrm{pm}$

$$
\begin{aligned}
& 98=\text { Pas de clientes PF } \\
& 99=\text { Pas d'observation }
\end{aligned}
$$

OBS 3. A quelle heure la première cliente PF a-t-elle été reçue aujourd'hui ? $\{\mid 3\}$ $\mathrm{am} / \mathrm{pm}$ $98=$ Pas de clientes PF $99=$ Pas d'observation

OBS 4. A quelle heure la dernière cliente PF a-t-elle été reçue aujourd'hui ? $\{14\}$ $\mathrm{am} / \mathrm{pm}$ $98=$ Pas de clientes PF $99=$ Pas d'observation

DEM 5. Quelle est l'heure de fermeture officielle de cette structure sanitaire ? $\{15\}$ $\mathrm{am} / \mathrm{pm}$

DEM 6. Pendant combien de jours par semaine des services PF sont-ils offerts dans cette structure sanitaire? $\{16\}$ jours par semaine

OBS 7. Y a-t-il un signe annonçant la disponibilité des services PF ? $\{17\}$

1 = A l'extérieur du bâtiment

2 = A l'intérieur du bâtiment

3 = A l'intérieur et à l'extérieur du bâtiment

$4=$ Pas de signe visible 
DEM 8. D'habitude, y a-t-il des services (lire de 1 - 17) disponibles dans cette unité de SMI/PF ou dans une autre unité ailleurs dans cette structure sanitaire ?

\begin{tabular}{|c|c|c|}
\hline & $\begin{array}{l}\text { A. Cochez si disponible } \\
\text { dans l'unité de SMI/PF }\end{array}$ & $\begin{array}{l}\text { B. Cochez si disponible } \\
\text { ailleurs dans la } \\
\text { structure sanitaire }\end{array}$ \\
\hline 1. Planification Familiale & $\{18 \mathrm{~A} 1\}$ & $\{18 \mathrm{~B} 1\}$ \\
\hline 2. Soins prénatals & $\{18 \mathrm{~A} 2\}$ & $\{18 \mathrm{~B} 2\}$ \\
\hline 3. Soins maternel/d'accouchement & $\{18 \mathrm{~A} 3\}$ & $\{18 \mathrm{~B} 3\}$ \\
\hline 4. Soins postnatals & $\{18 \mathrm{~A} 4\}$ & \{18B4\} \\
\hline 5. Counseling/IEC sur le VIH/SIDA & $\{18 \mathrm{~A} 5\}$ & $\{1885\}$ \\
\hline 6. Test de VIH/SIDA & $\{18 \mathrm{~A} 6\}$ & $\{1886\}$ \\
\hline 7. Counseling/IEC sur d'autres MST & $\{18 \mathrm{~A} 7\}$ & $\{18 B 7\}$ \\
\hline 8. Diagnostic d'autres MST & $\{18 \mathrm{AB}\}$ & $\{1888\}$ \\
\hline 9. Traitement d'autres MST & $\{18 \mathrm{~A} 9\}$ & $\{1889\}$ \\
\hline 10. Vaccination des enfants & $\{18 \mathrm{~A} 10\}$ & $\{18 \mathrm{~B} 10\}$ \\
\hline 11. Surveillance de la croissance de l'enfant & $\{\mid 8 A 11\}$ & $\{18 \mathrm{~B} 11\}$ \\
\hline 12. Consultation pour infécondité & $\{\mid 8 A 12\}$ & $\{18 \mathrm{~B} 12\}$ \\
\hline 13. Thérapie de réhydratation orale & $\{18 \mathrm{~A} 13\}$ & $\{\mid 8 B 13\}$ \\
\hline 14. Traitement de l'avortement incomplet & $\{18 \mathrm{~A} 14\}$ & $\{18 \mathrm{~B} 14\}$ \\
\hline 15. Counseling en matière de nutrition & $\{\mid 8 A 15\}$ & $\{18 \mathrm{~B} 15\}$ \\
\hline 16. Services curatifs — clientes & $\{\mid 8 A 16\}$ & $\{\mid 8 B 16\}$ \\
\hline 17. Services curatifs - enfants & $\{18 \mathrm{~A} 17\}$ & $\{18 B 17\}$ \\
\hline
\end{tabular}


OBS 9. L'unité de SMI/PF dispose-t-elle des équipements suivants le jour de la visite :

\begin{tabular}{|l|l|}
\hline & Cochez si présent \\
\hline 1. Eau courante & $\{19 \times 1\}$ \\
\hline 2. Electricité & $\{19 \times 2\}$ \\
\hline 3. Toilettes fonctionnelles / latrines disponibles pour les clientes & $\{19 \times 3\}$ \\
\hline 4. Assez de places pour s'asseoir pour les clientes & $\{19 \times 4\}$ \\
\hline 5. & $\{19 \times 5\}$ \\
\hline 6. & $\{19 \times 6\}$ \\
\hline
\end{tabular}

\section{DOTATION EN PERSONNEL}

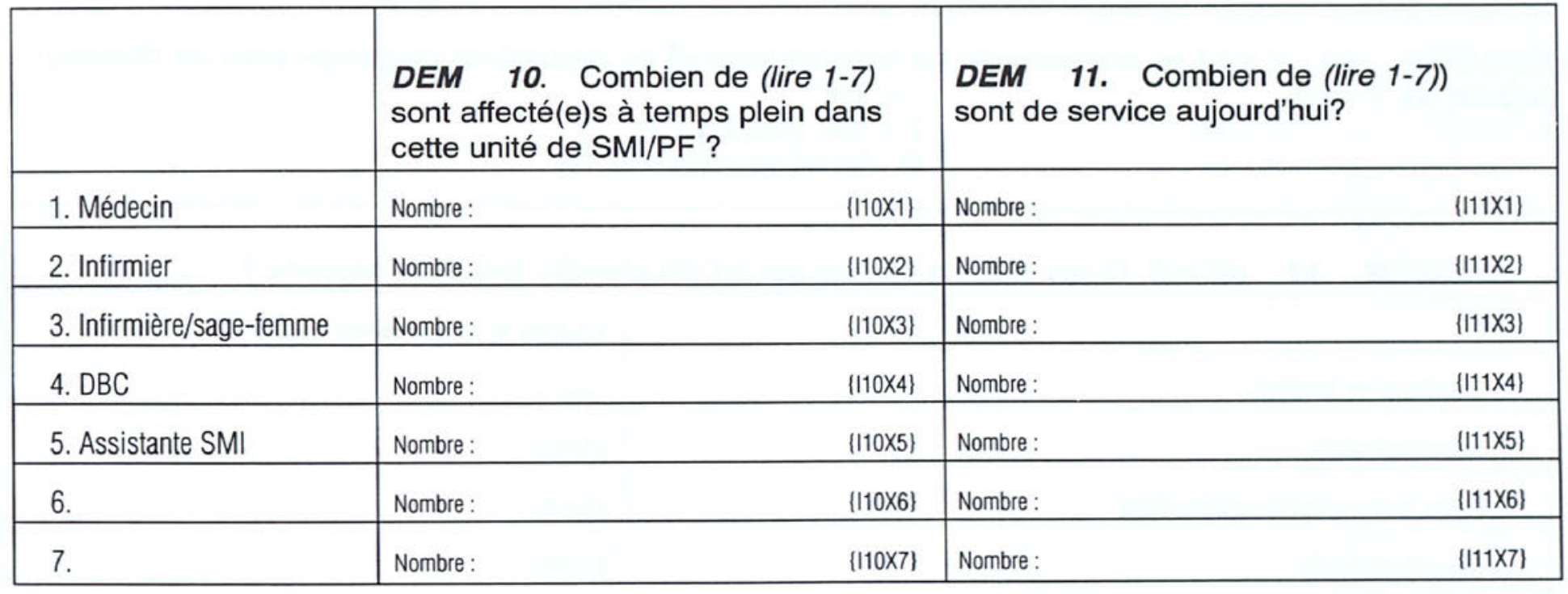




\section{MATÉRIELS ET ACTIVITÉS D'IEC}

OBS 12. Lesquels des matériels d'IEC sont disponibles dans l'unité de SMI/PF sur les thèmes suivants ?

\begin{tabular}{|l|l|l|l|}
\hline & $\begin{array}{l}\text { A. Cochez si une } \\
\text { boîte à images est } \\
\text { disponible }\end{array}$ & $\begin{array}{l}\text { B. Cochez si une } \\
\text { brochure/un dépliant } \\
\text { est disponible }\end{array}$ & $\begin{array}{l}\text { C. Cochez si une } \\
\text { affiche est } \\
\text { disponible }\end{array}$ \\
\hline 1. Planification familiale & $\{\mid 12 \mathrm{~A} 1\}$ & $\{[12 \mathrm{~B} 1\}$ & $\{[12 \mathrm{C} 1\}$ \\
\hline 2. Soins prénatals/postnatals & $\{\mid 12 \mathrm{~A} 2\}$ & $\{[12 \mathrm{~B} 2\}$ & $\{[12 \mathrm{C} 2\}$ \\
\hline 3. Services d'accouchement & $\{\mid 12 \mathrm{~A}\}\}$ & $\{[12 \mathrm{~B} 3\}$ & $\{[12 \mathrm{C} 3\}$ \\
\hline 4. VIH/SIDA & $\{\mid 12 \mathrm{~A} 4\}$ & $\{[12 \mathrm{~B} 4\}$ & $\{[12 \mathrm{C} 4\}$ \\
\hline 5. Autres MST & $\{\mid 12 \mathrm{~A}\}\}$ & $\{[12 \mathrm{~B} 5\}$ & $\{[12 \mathrm{C} 5\}$ \\
\hline 6. Bien-être de l'enfant & $\{\mid 12 \mathrm{~A} 6\}$ & $\{[12 \mathrm{~B} 6\}$ & $\{[12 \mathrm{C} 6\}$ \\
\hline 7. Nutrition & $\{\mid 12 \mathrm{~A} 7\}$ & $\{[12 \mathrm{~B} 7\}$ & $\{[12 \mathrm{C} 7\}$ \\
\hline
\end{tabular}

OBS/DEM 13. Y a-t-il eu une causerie sur la santé (exposé ou discussions de groupe avec les clientes) aujourd'hui ? \{113\}

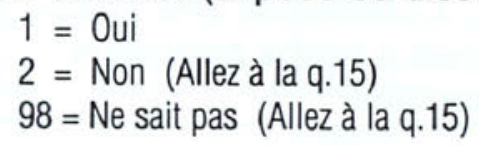

OBS/DEM 14. (Si oui) Quels sont les thèmes qui ont été abordés lors de la causerie ?

\begin{tabular}{|c|c|}
\hline & Cochez si le thème est abordé \\
\hline 1. Planification familiale & $\{\mid 14 \times 1\}$ \\
\hline 2. Soins prénatals & $\{114 \times 2\}$ \\
\hline 3. Soins maternels/d'accouchement & $\{\mid 14 \times 3\}$ \\
\hline 4. Soins postnatals & $\{114 \times 4\}$ \\
\hline 5. VIH/SIDA & $\{114 \times 5\}$ \\
\hline 6. Autres MST & $\{114 \times 6\}$ \\
\hline 7. Vaccination des enfants & $\{114 \times 7\}$ \\
\hline 8. Surveillance de la croissance infantile & $\{114 \times 8\}$ \\
\hline 9. Consultation pour infécondité & $\{\mid 14 \times 9\}$ \\
\hline 10. Thérapie de réhydratation orale & $\{114 \times 10\}$ \\
\hline 11. Traitement de l'avortement incomplet & $\{114 X 11\}$ \\
\hline 12. Counseling en matière de nutrition & $\{114 \times 12\}$ \\
\hline 13. Services curatifs — clientes & $\{114 \times 13\}$ \\
\hline 14. Services curatifs - enfants & $\{114 \times 14\}$ \\
\hline 15. Allaitement & $\{114 \times 15\}$ \\
\hline 16. Autre: & $\{\mid 14 X 16\}$ \\
\hline
\end{tabular}




\section{LIEU DES EXAMENS MÉDICAUX}

\begin{tabular}{|l|l|}
\hline \multicolumn{2}{|l|}{ OBS 15. Les conditions suivantes sont-elles observées dans le lieu des examens médicaux ? } \\
\hline & Cochez si observées \\
\hline 1. Protection contre l'écoute indiscrète & $\{\{15 \times 1\}$ \\
\hline 2. Protection contre les regards indiscrets & $\{\mid 15 \times 2\}$ \\
\hline 3. Propreté & $\{\mid 15 \times 3\}$ \\
\hline 4. Lumière appropriée ${ }^{\star \star}$ & $\{\mid 15 \times 4\}$ \\
\hline 5. Eau en quantité suffisante & $\{115 \times 5\}$ \\
\hline
\end{tabular}

* "Propreté" signifie les sols balayés et essuyés au début de la journée, pas de poussière sur les fenêtres ou sur les tables

** "Lumière appropriée" signifie lumière électrique qui fonctionne ou lumière naturelle suffisante.

*** "Eau en quantité suffisante" signifie eau en quantité suffisante pour se laver les mains et les équipements. 


\section{INVENTAIRE DES ÉQUIPEMENT ET DES PRODUITS}

OBS 16. Cochez si les types d'équipements suivants sont disponibles et fonctionnels dans l'unité de SMI/PF, et/ou dans la salle de stock pour les services SMI/PF

\begin{tabular}{|c|c|}
\hline & Cochez si disponible \\
\hline 1. Matériel de stérilisation dans l'unité de SMI/PF & $\{116 \times 1\}$ \\
\hline 2. Matériel de stérilisation hors de l'unité de SMI/PF (partagé) & $\{116 \times 2\}$ \\
\hline 3. Lampes baladeuses/gynécologiques/torche & $\{116 \times 3\}$ \\
\hline 4. Appareil à tension & $\{116 \times 4\}$ \\
\hline 5. Pèse personne pour adulte & $\{116 \times 5\}$ \\
\hline 6. Pèse bébé & $\{116 \times 6\}$ \\
\hline 7. Ciseaux & $\{116 \times 7\}$ \\
\hline 8. Lotions antiseptiques & $\{116 \times 8\}$ \\
\hline 9. Stéthoscopes & $\{116 \times 9\}$ \\
\hline 10. Réfrigérateur pour le PEV (vaccination) & $\{16 \times 10\}$ \\
\hline 11. Table d'examen & $\{116 \times 11\}$ \\
\hline 12. Thermomètre & $\{116 \times 12\}$ \\
\hline 13. Aiguilles & $\{\mid 16 \times 13\}$ \\
\hline 14. Seringues & $\{16 \times 14\}$ \\
\hline 15. Microscope & $\{16 \times 15\}$ \\
\hline 16. Coton hydrophile & $\{16 \times 16\}$ \\
\hline 17. Compresse & $\{16 \times 17\}$ \\
\hline 18. Détol & $\{116 \times 18\}$ \\
\hline
\end{tabular}

OBS 17. Comptez le nombre de types d'équipements suivants disponibles et fonctionnels dans l'unité de SMI/PF et/ou dans la salle de stock pour les services SMI/PF.
1. Pinces à éponge
2. Sondes utérines
3. Spécula
4. Ténacula
5. Gants à usage multiple (nombre de pairs)
6. Gants à usage unique (nombre de pairs)

\section{Comptez}

Nombre : $\{117 \times 1\}$

Nombre : $\{117 \times 2\}$

Nombre : $\{117 \times 3\}$

Nombre : $\{117 \times 4\}$

Nombre : $\{117 \times 5\}$

Nombre : $\{117 \times 6\}$ 


\begin{tabular}{|c|c|c|c|}
\hline & $\begin{array}{l}\text { DEM 18. D'habitude } \\
\text { l'unité de SMI/PF fournit- } \\
\text { elle chacune des } \\
\text { méthodes contraceptives } \\
\text { suivantes? } \\
\text { (Lire 1-9 et cochez si } \\
\text { fournie) }\end{array}$ & $\begin{array}{l}\text { OBS 19. (Si la } \\
\text { méthode est fournie } \\
\text { d'habitude) Vérifiez } \\
\text { physiquement et cochez } \\
\text { si les produits sont } \\
\text { disponibles aujourd'hui. }\end{array}$ & $\begin{array}{l}\text { DEM 20. (Si la } \\
\text { méthode est fournie } \\
\text { d'habitude) Y a-t-il eu } \\
\text { une rupture de stock au } \\
\text { cours des six derniers } \\
\text { mois? } \\
\text { (Cochez si oui) }\end{array}$ \\
\hline 1. Pilule combinée & $\{118 \times 1\}$ & $\{119 \times 1\}$ & $\{120 \times 1\}$ \\
\hline 2. Pilule progestative & $\{118 \times 2\}$ & $\{119 \times 2\}$ & $\{120 \times 2\}$ \\
\hline 3. DIU & $\{118 \times 3\}$ & $\{119 \times 3\}$ & $\{120 \times 3\}$ \\
\hline 4. Injectable & $\{118 \times 4\}$ & $\{\mid 19 \times 4\}$ & $\{120 \times 4\}$ \\
\hline 5. NORPLANT ${ }^{\oplus}$ & $\{118 \times 5\}$ & $\{119 \times 5\}$ & $\{120 \times 5\}$ \\
\hline 6. Préservatif & $\{118 \times 6\}$ & $\{\mid 19 \times 6\}$ & $\{120 \times 6\}$ \\
\hline 7. Diaphragme & $\{\mid 18 \times 7\}$ & $\{\mid 19 \times 7\}$ & $\{120 \times 7\}$ \\
\hline 8. Spermicide & $\{118 \times 8\}$ & $\{119 \times 8\}$ & $\{120 \times 8\}$ \\
\hline 9. Autre : & $\{118 \times 9\}$ & $\{119 \times 9\}$ & \{120X9\} \\
\hline
\end{tabular}

DEM 21. La structure sanitaire (y compris l'unité de SMI/PF) fournit-elle d'habitude les services suivants ou le counseling ?

\begin{tabular}{|l|l|}
\hline & Cochez si fourni (Lire 1-6) \\
\hline 1. Ligature des trompes & $\{121 \times 1\}$ \\
\hline 2. Vasectomie & $\{121 \times 2\}$ \\
\hline 3. Counseling sur la planification familiale naturelle & $\{121 \times 3\}$ \\
\hline 4. Counseling sur l'allaitement maternel exclusif (MAMA) & $\{121 \times 4\}$ \\
\hline 5. Counseling sur les méthodes (mixtes) & $\{121 \times 5\}$ \\
\hline 6. Contraception d'urgence & $\{121 \times 6\}$ \\
\hline
\end{tabular}




\section{SERVICES DE DÉPISTAGE ET DE DIAGNOSTIC}

$1=$ Oui

$2=$ Non (Allez à la q.24)

DEM 23. Y a-t-il des tests de (lire 1-7) disponibles dans cette unité de SMI/PF ou dans cette structure sanitaire, ou bien des prélèvements de clientes, ou les clientes elles-mêmes sont-ils envoyés ailleurs?

\begin{tabular}{|l|l|l|}
\hline & $\begin{array}{l}\text { A. Cochez si le test est } \\
\text { disponible dans cette } \\
\text { structure sanitaire }\end{array}$ & $\begin{array}{l}\text { B. Cochez si des } \\
\text { prélèvements ou les } \\
\text { clientes sont envoyés } \\
\text { ailleurs }\end{array}$ \\
\hline 1. Syphilis & $\{[123 \mathrm{~A}\}\}$ & $\{\{123 \mathrm{~B} 1\}$ \\
\hline 2. Gonococcie & $\{[123 \mathrm{~A} 2\}$ & $\{\{123 \mathrm{~B} 2\}$ \\
\hline 3. Chlamydia & $\{\mid 123 \mathrm{~A} 3\}$ & $\{\mid 123 \mathrm{~B} 3\}$ \\
\hline 4. VIH & $\{[123 \mathrm{~A} 4\}$ & $\{\mid 123 \mathrm{~B} 4\}$ \\
\hline 5. Candida & $\{[123 \mathrm{~A} 5\}$ & $\{\mid 123 \mathrm{~B} 5\}$ \\
\hline 6. Cancer du col de l'utérus (test de Papanicolaou) & $\{[123 \mathrm{~A} 6\}$ & $\{\mid 123 \mathrm{~B} 6\}$ \\
\hline 7. Grossesse & $\{\mid 123 \mathrm{~A}\}\}$ & $\{\mid 123 \mathrm{~B} 7\}$ \\
\hline
\end{tabular}

\section{SERVICES DE VACCINATION}

\begin{tabular}{|c|c|c|}
\hline & $\begin{array}{l}\text { DEM 24. Cette structure sanitaire } \\
\text { offre-t-elle d'habitude les vaccins } \\
\text { suivants? } \\
\text { (Lire } 1-6 \text { et cocher si les services sont offerts) }\end{array}$ & $\begin{array}{l}D E M \quad 25 . \quad \text { (Si le vaccin est fourni) } \\
\text { Les antigènes ont-ils connu une } \\
\text { rupture de stock au cours des six } \\
\text { derniers mois? } \\
\text { (Cochez si oui) }\end{array}$ \\
\hline 1. BCG & $\{\mid 124 \times 1\}$ & $\{1125 \times 1\}$ \\
\hline 2. Polio & $\{1124 \times 2\}$ & $\{1125 \times 2\}$ \\
\hline 3. DPT & $\{\mid 124 \times 3\}$ & $\{\mid 125 \times 3\}$ \\
\hline 4. Rougeole & $\{1124 \times 4\}$ & $\{1125 \times 4\}$ \\
\hline 5. Hépatite B & $\{\mid 124 \times 5\}$ & $\{1125 \times 5\}$ \\
\hline $\begin{array}{l}\text { 6. Tétanos pour les clientes } \\
\text { des soins prénatals }\end{array}$ & $\{1124 \times 6\}$ & $\{1125 \times 6\}$ \\
\hline
\end{tabular}




\section{GESTION DES PRODUITS}

\begin{tabular}{|l|l|l|}
\hline & $\begin{array}{l}\text { DEM 26. Existe-t-il un inventaire } \\
\text { écrit des produits et des provisions } \\
\text { suivants? } \\
\text { (Lire de 1-4 et cochez si oui) }\end{array}$ & $\begin{array}{l}\text { OBS 27. (S'il existe un inventaire) } \\
\text { Est-il à jour, lisible et complet? }\end{array}$ \\
\hline 1. Produits contraceptifs & $\{126 \mathrm{X} 1\}$ & $\{\mid 27 \times 1\}$ \\
\hline $\begin{array}{l}\text { 2. Médicaments pour } \\
\text { le traitement des MST }\end{array}$ & $\{126 \times 2\}$ & $\{127 \times 2\}$ \\
\hline 3. Vaccins & $\{126 \times 3\}$ & $\{127 \times 3\}$ \\
\hline 4. Autres médicaments & $\{126 \times 4\}$ & $\{127 \times 4\}$ \\
\hline
\end{tabular}

\begin{tabular}{|l|l|l|}
\hline & $\begin{array}{l}\text { DEM 28. Les produits suivants } \\
\text { sont-ils stockés selon la date de } \\
\text { péremption ? En d'autres termes, } \\
\text { existe-t-il un système de "premier } \\
\text { périmé premier sorti ?" } \\
\text { (Lire 1-4 et cochez si oui) }\end{array}$ & $\begin{array}{l}\text { OBS 29. Pour chaque produit, les } \\
\text { lieux de stockage sont-ils protégés de } \\
\text { la pluie, du soleil, des températures } \\
\text { défavorables, des rats et des insectes } \\
\text { nuisibles? } \\
\text { (Lire de 1-4 et cochez si oui) }\end{array}$ \\
\hline 1. Produits contraceptifs & $\{\mid 28 \times 1\}$ & $\{129 \times 1\}$ \\
\hline $\begin{array}{l}\text { 2. Médicaments pour } \\
\text { le traitement des MST }\end{array}$ & $\{\mid 28 \times 2\}$ & $\{[29 \times 2\}$ \\
\hline 3. Vaccins & $\{\mid 28 \times 3\}$ & $\{[29 \times 3\}$ \\
\hline 4. Autres médicaments & $\{\mid 28 \times 4\}$ & $\{\mid 29 \times 4\}$ \\
\hline
\end{tabular}




\section{SERVICES DES STATISTIQUES ET RÉDACTION DES RAPPORTS}

DEM 30. Comment les fiches des clientes sont-elles gardées dans cette structure sanitaire ? $\{130\}$

1 = Gardées dans la clinique

2 = Gardées par la cliente (allez à la q.32)

$3=$ Pas de fiches (allez à la q.32)

4 = Autre :

[I3OR\}

OBS 31. Quel est l'état du système des fiches des clientes ? \{|31\}

1 = Bien ordonné

2 = Partiellement ordonné, encore utilisable

3 = Désordonné, pas utilisable

DEM 32. Existe-t-il un seul registre des activités quotidiennes pour tous les services SMI/PF fournis par la structure sanitaire ? $\{132\}$

$1=$ Oui pour tous les services

2 = Oui pour quelques services

3 = Registre séparé pour chacun des services

4 = Pas de registre quotidien des activités

DEM. 33 Les rapports de statistiques des services suivants sont-ils envoyés à un superviseur ou à une unité supérieure? (Lisez de 1-3)

\begin{tabular}{|l|l|}
\hline & Cochez si des rapports sont envoyés \\
\hline 1. SMI & $\{\mid 33 \times 1\}$ \\
\hline 2. PF & $\{\mid 33 \times 2\}$ \\
\hline 3. MSTNIH/SIDA & $\{\mid 33 \times 3\}$ \\
\hline
\end{tabular}




\section{GESTION ET SUPERVISION}

DEM 34. Au cours des six derniers mois, combien de fois un superviseur est-il venu dans cette unité de SMI/PF pour des raisons de supervision ? $\{134\}$

DEM 35. En visitant cette structure sanitaire, qu'est-ce que le superviseur fait ? (Ne pas lire, mais susciter davantage de réponses en demandant, "D'autres actions?")

\begin{tabular}{|c|c|}
\hline & Cochez si l'action est mentionnée \\
\hline 1. Observe la prestation de différents services & $\{\mid 35 X 1\}$ \\
\hline 2. Observe seulement le service dont il/elle est responsable & $\{135 \times 2\}$ \\
\hline 3. S'informe des problèmes du service & $\{135 \times 3\}$ \\
\hline 4. Examine les statistiques & $\{135 \times 4\}$ \\
\hline 5. Fait des suggestions pour des améliorations & $\{135 \times 5\}$ \\
\hline 6. Fait l'éloge du bon travail & $\{135 \times 6\}$ \\
\hline 7. Autre: & $\{135 \times 7\}$ \\
\hline
\end{tabular}

\section{STATISTIQUES DES SERVICES}

Compiler les statistiques des clientes sur une période de 12 mois consécutifs au cours des 24 derniers mois. Dans les cas où on ne peut pas obtenir une période de 12 mois consécutifs, utiliser la période continue la plus longue pour laquelle ces statistiques sont disponibles et enregistrer le nombre de mois dans la colonne $\mathrm{C}$.

OBS 36. Combien de clientes ont reçu les services suivants au cours des 12 derniers mois ? (99999 = pas de données disponibles)

\begin{tabular}{|c|c|c|c|}
\hline & A. Nouvelles clientes & B. Anciennes clientes & $\begin{array}{l}\text { C. A partir du nombre } \\
\text { de mois de statistiques } \\
\text { continues }\end{array}$ \\
\hline 1. Planification familiale & $\{\mid 36 \mathrm{~A} 1\}$ & $\{\mid 36 \mathrm{~B} 1\}$ & $\{136 \mathrm{C} 1\}$ \\
\hline 2. Services prénatals & \{136A2\} & $\{\mid 36 \mathrm{~B} 2\}$ & $\{136 \mathrm{C} 2\}$ \\
\hline 3. Services d'accouchement & $\{\mid 36 \mathrm{~A} 3\}$ & [І36B3\} & $\{136 \mathrm{C} 3\}$ \\
\hline 4. Services postnatals & $\{\mid 36 \mathrm{~A} 4\}$ & $\{\mid 36 \mathrm{~B} 4\}$ & $\{136 \mathrm{C} 4\}$ \\
\hline 5. Services de VIH/SIDA & $\{\mid 36 \mathrm{~A} 5\}$ & $\{136 \mathrm{~B} 5\}$ & $\{136 \mathrm{C} 5\}$ \\
\hline 6. Services de MST & $\{136 \mathrm{~A} 6\}$ & $\{136 \mathrm{~B} 6\}$ & $\{136 \mathrm{C} 6\}$ \\
\hline 7. Bien-être de l'enfant & $\{\mid 36 \mathrm{~A} 7\}$ & $\{\mid 36 B 7\}$ & $\{136 \mathrm{C} 7\}$ \\
\hline
\end{tabular}

OBS 37. Indiquer le dernier mois et l'année mentionnés dans le tableau ci-dessus.

mois \{137X1\} Année $\{137 \times 2\}$ 
DEM 38. Quel est le coût de chaque méthode ou service pour la cliente ? (Lire de 1 - 26)

\begin{tabular}{|c|c|c|}
\hline & A. Frais de Consultation & $\begin{array}{l}\text { B. Produit/ } \\
\text { test/procédure }\end{array}$ \\
\hline 1. Pilule combinée & $\{\mid 38 A 1\}$ & $\{\mid 38 \mathrm{~B} 1\}$ \\
\hline 2. Pilule progestative & $\{\mid 38 \mathrm{~A} 2\}$ & $\{\mid 38 \mathrm{~B} 2\}$ \\
\hline 3. DIU & $\{\mid 38 \mathrm{~A} 3\}$ & $\{\mid 38 \mathrm{~B} 3\}$ \\
\hline 4. Injectable & $\{\mid 38 \mathrm{~A} 4\}$ & $\{\mid 38 B 4\}$ \\
\hline 5. NORPLANT ${ }^{\oplus}$ & $\{\mid 38 A 5\}$ & $\{138 \mathrm{~B} 5\}$ \\
\hline 6. Préservatif & $\{\mid 38 A 6\}$ & $\{138 \mathrm{~B} 6\}$ \\
\hline 7. Diaphragme & $\{\mid 38 A 7\}$ & $\{138 \mathrm{~B} 7\}$ \\
\hline 8. Spermicide & $\{\mid 38 A 8\}$ & $\{138 \mathrm{~B} 8\}$ \\
\hline 9. Ligature des trompes (ML/AL) & $\{138 \mathrm{~A} 9\}$ & $\{138 \mathrm{~B} 9\}$ \\
\hline 10. Vasectomie & $\{\mid 38 A 10\}$ & $\{138 \mathrm{~B} 10\}$ \\
\hline 11. Counseling en PF naturelle & $\{\mid 38 A 11\}$ & $\{138 \mathrm{~B} 11\}$ \\
\hline 12. Counseling en MAMA & $\{\mid 38 A 12\}$ & $\{\mid 38 B 12\}$ \\
\hline 13. Soins prénatals & $\{138 \mathrm{~A} 13\}$ & $\{\mid 38 B 13\}$ \\
\hline 14. Soins maternels/services d'accouchement & $\{138 \mathrm{~A} 14\}$ & $\{138814\}$ \\
\hline 15. Soins postnatals & $\{138 \mathrm{~A} 15\}$ & $\{138 B 15\}$ \\
\hline 16. Counseling en IEC VIH/SIDA & $\{138 \mathrm{~A} 16\}$ & $\{138 B 16\}$ \\
\hline 17. Test de VIH/SIDA & $\{\mid 38 A 17\}$ & $\{\mid 38 B 17\}$ \\
\hline 18. Counseling en IEC pour d'autres MST & $\{\mid 38 \mathrm{~A} 18\}$ & $\{\mid 38 B 18\}$ \\
\hline 19. Diagnostic d'autres MST & $\{\mid 38 A 19\}$ & $\{\mid 38 B 19\}$ \\
\hline 20. Traitement d'autres MST & $\{138 \mathrm{~A} 20\}$ & $\{138 \mathrm{~B} 20\}$ \\
\hline 21. Vaccination des enfants & \{138A21\} & $\{138 \mathrm{~B} 21\}$ \\
\hline 22. Surveillance de la croissance de l'enfant & $\{138 \mathrm{~A} 22\}$ & $\{138 \mathrm{~B} 22\}$ \\
\hline 23. Consultation pour infécondité & $\{\mid 38 \mathrm{~A} 23\}$ & $\{\mid 38 B 23\}$ \\
\hline 24. Thérapie de réhydratation orale & $\{138 \mathrm{~A} 24\}$ & $\{\mid 38 B 24\}$ \\
\hline 25. Traitement de l'avortement incomplet & $\{\mid 38 A 25\}$ & $\{\mid 38 \mathrm{~B} 25\}$ \\
\hline 26. Counseling en nutrition & $\{\mid 38 \mathrm{~A} 26\}$ & $\{\mid 38826\}$ \\
\hline
\end{tabular}


Inventaire : guide question par question Le remplissage de cet instrument peut nécessiter toute une journée pour une personne. Il devrait commencer tôt après l'arrivée. Si l'enquêteur de terrain n'est pas un infirmier, il/elle aura besoin de l'aide d'un infirmier pour faire l'inventaire de l'équipement et des provisions. Le processus de conduite de l'inventaire nécessite qu'on fasse des observations et que l'on pose des questions au responsable des services PF dans le PPS. Les abréviations DEM ou OBS se trouvant à gauche des numéros des différents volets indiquent au chercheur s'il faut demander ou observer la situation pour obtenir l'information recherchée. Il faut noter que puisque le personnel chargé des services PF sera interviewé avec l'instrument d'interview du personnel, il serait nécessaire pour les différents membres de l'équipe de recherche sur le terrain de coordonner le temps pour chaque instrument et d'être sensibles au fait de ne pas surcharger le personnel.

\section{Page de couverture}

Avant de commencer l'inventaire, compléter toutes les informations de la page de couverture (sauf le Numéro du questionnaire. Il sera rempli à la fin de la collecte des données par votre Chef d'équipe). Cette page est similaire aux pages de couverture de tous les autres questionnaires. Il est très important que les codes de la structure sanitaire, du district et du village/ville soient corrects. Demandez à votre Chef d'équipe si vous n'êtes pas sûr. Demandez au chef du PPS d'identifier pour vous le type de structure sanitaire, le type de secteur et la localité. S'il y a une confusion, demander à votre Chef d'équipe.

Ecrivez (ne pas signer) votre nom dans l'encadré qui se trouve au bas de la page. Lorsque vous finissez l'inventaire, votre Chef d'équipe le révisera et signera en bas de votre nom.

\section{Quelle est l'heure d'ouverture officielle de cette structure sanitaire ?}

2. A quelle heure la première cliente de planification familiale est-elle arrivée aujourd'hui ?
3. A quelle heure la première cliente PF a-telle été reçue aujourd'hui ?

Ces questions détermineront si le PPS est ouvert en réalité et fournit des services à I'heure d'ouverture officielle. Essayez d'arriver dans le PPS avant son heure d'ouverture officielle. Enregistrez l'heure d'arrivée de la première cliente de planification familiale et l'heure à laquelle elle a commencé sa consultation avec un prestataire. Le fait d'avoir une causerie sanitaire n'est pas considéré comme étant "reçue" par un prestataire. En enregistrant les temps, rassurez-vous d'encercler "am" (matin) ou "pm (après-midi)."

4. A quelle heure la dernière cliente PF a-telle été reçue aujourd'hui ?

Cette question nécessite que vous restiez dans le PPS jusqu'à la fin de la prestation de services, pour être sûr d'avoir identifié la dernière cliente de la journée. S'il n'y a eu qu'une seule cliente, enregistrez l'heure à laquelle elle a été reçue dans les questions 3 et 4 .

5. Quelle est l'heure de fermeture officielle de cette structure sanitaire ?

Demandez au responsable des services PF l'heure de fermeture officielle. Cette question et la question 4 montreront si les services sont offerts pendant les heures déclarées.

6. Pendant combien de jours des services PF sont-ils offerts dans cette structure sanitaire par semaine?

Demandez au responsable des services PF combien de jours les services PF sont offerts par semaine. Les journées incomplètes sont comptées comme des jours entiers. C'est-àdire si des services sont offerts deux heures par jour pendant trois jours, il faut enregistrer 3. Si les services sont offerts huit heures pendant un jour de la semaine, il faut enregistrer 1 . 
7. $Y$ a-t-il un panneau annonçant la disponibilité des services PF ?

Les panneaux aident les clientes à comprendre qu'elles peuvent obtenir des services PF dans le PPS. Cherchez un qui indique que des services PF sont disponibles dans cette structure sanitaire. Parfois, le panneau est à l'extérieur près de la route et dans d'autres cas il est à l'intérieur du bâtiment du PPS. S'il y a plusieurs panneaux, il faut les cocher de manière appropriée.

8. D'habitude, y a-t-il des (plusieurs services) disponibles dans cette unité de SMI/PF ou ailleurs dans cette structure sanitaire ? Ces questions aideront à évaluer la gamme de services offerts dans le PPS. Demandez au responsable des services PF si chacun des services est disponible d'habitude, et dans quel endroit de la structure sanitaire. "D'habitude disponible" veut dire offert au moins trois jours par semaine. Si un service est disponible à la fois dans cette unité de SMI/PF et ailleurs dans la structure sanitaire, il faut cocher les deux. S'il n'est disponible qu'ailleurs dans la structure sanitaire, il faut cocher la colonne B, même s'il n'existe pas un système standard de référence.

\section{L'unité de SMI/PF dispose-t-elle des} équipements (infrastructure de base) suivants le jour de la visite?

Les infrastructures de base d'un PPS ont un impact tant sur sa capacité de fournir des services de haute qualité que sur la satisfaction des clientes par rapport aux services. Cochez si le PPS dispose d'eau courante, d'électricité, des toilettes fonctionnelles et suffisamment de places pour s'asseoir pour les clientes. Vérifiez l'eau : coule-t-elle en réalité ? Parfois, il peut y avoir un robinet et des tuyaux mais pas d'eau. $\mathrm{Si}$ l'eau ne coule pas, il ne faut pas cocher l'encadré. Mettez le contact d'une lampe. S'il n'y a pas de lumière, ne cochez pas l'encadré même si le personnel vous dit qu'il s'agit d'une coupure pour la seule journée de la visite. Vérifiez les toilettes pour voir si elles sont bien fonctionnelles. Selon votre propre jugement, si elles sont sales ou inutilisables, il ne faut pas cocher l'encadré correspondant.

10. Combien de (différents types de personnels) sont désignés pour travailler à temps plein dans cette unité du PPS ? Le système de dotation en personnel d'un PPS peut indiquer le niveau de services qui pourraient ou qui devraient être fournis dans ce PPS. Demandez au responsable des services PF le nombre de chaque type de personnel qui travaille à temps plein dans cette unité de SMI/PF. Il ne faut pas inclure les postes qui sont établis mais qui sont actuellement vacants. N'inclure que le personnel qui fournit des services PF. Les membres du personnel fournissent des services PF s'ils font l'une des activités suivantes :

- conseillent les clientes en les informant sur les méthodes contraceptives ou en les référant vers d'autres PPS pour recevoir des services;

- font la stérilisation;

- font les injections ou posent les DIU ou le NORPLANT ${ }^{\circ}$;

- prescrivent les pilules;

- donnent les préservatifs, la mousse ou les gelées aux clientes.

\section{Combien de (différents types de} personnel) sont de service aujourd'hui ? En guise de suivi à la question précédente, demandez le nombre des membres du personnel identifiés dans la première colonne qui sont de service aujourd'hui. Si un membre du personnel est supposé être de service mais est absent aujourd'hui pour cause de maladie ou tout autre problème, il ne faut pas le compter comme étant "de service." Cette information fera l'évaluation de la capacité 
pratique journalière à fournir les services (par rapport à la capacité formelle mesurée par la question précédente).

\section{Lesquels des matériels IEC sont disponibles dans l'unité de SMI/PF sur les thèmes suivants ?}

Les matériels IEC peuvent être des outils utiles à la communication, et leur présence renforce la qualité des services. Pour chaque élément de cette question, il est important que vous vérifiez en effet qu'un matériel IEC particulier est présent. Pour le vérifier, vous devez le voir en réalité. Regardez s'il y a des affiches sur les murs ou les portes du PPS. Demandez au responsable des services PF si la structure sanitaire dispose de boîtes à image, de dépliants ou de brochures. Cochez l'encadré correspondant seulement si vous voyez les matériels. Si le PPS ne dispose que de quelques dépliants ou brochures-pas en quantité suffisante pour être distribués aux clientes-il faut les cocher comme disponibles puisqu'ils peuvent être utilisés pendant le counseling des clientes.

\section{Y a-t-il eu une causerie sur la santé (exposé ou discussion de groupe avec les clientes) aujourd'hui ?}

14. (Si oui) Quels sont les thèmes qui ont été abordés dans la causerie?

Ces questions évaluent si les clientes ont accès à l'information sur la PF et la SR en dehors des consultations avec les prestataires. Généralement, les causeries sur la santé sont faites au début de la journée par une infirmière/sage-femme. Pour déterminer s'il y a eu une causerie sur la santé, vous devez arriver dans le PPS avant son ouverture pour le travail de la journée. Si vous n'avez pas vu en réalité qu'il y a eu une causerie sanitaire à cause du fait que vous êtes arrivé en retard, il faut encercler 98 . Si vous avez en réalité écouté la causerie sanitaire, encerclez 1 et cochez les thèmes de la question 14 qui ont été abordés dans la causerie.

\section{Les conditions suivantes sont-elles} observées dans le lieu des examens médicaux?

Les conditions de la salle d'examen peuvent affecter de manière drastique aussi bien la qualité des soins fournis que la satisfaction des clientes par rapport au PPS. Vous utiliserez votre propre jugement en ce qui concerne l'intimité auditive et visuelle, la propreté, la lumière et l'eau suffisantes. Voici quelques directives:

- "intimité auditive" veut dire qu'une conversation entre une cliente et une infirmière/sage-femme ou un médecin ne peut pas être comprise par les autres clientes. Il se peut que les autres clientes voient qu'une conversation se déroule, mais ne peuvent pas entendre ce qu'on dit. S'il n'y a pas de clientes le jour de la visite et que vous ne pouvez pas juger le niveau d'intimité, écrivez 98 dans l'encadré.

- "intimité visuelle" veut dire que les autres clientes ne peuvent pas voir l'interaction entre une cliente et un médecin ou une infirmière/sage-femme. Généralement, cela signifie qu'il existe une salle d'examen séparée qui est privée ou qu'il $\mathrm{y}$ a un endroit qui a un rideau ou une autre partition qui empêche les autres clientes de voir ce qui se passe. S'il n'y a pas de clientes le jour de la visite et que vous ne pouvez pas juger le niveau d'intimité, écrivez 98 dans l'encadré.

- "propre" veut dire qu'au début de la journée du nouveau linge est utilisé sur les lits et les tables d'examen et que de nouvelles serviettes sont fournies. Il signifie aussi que les sols sont balayés et essuyés et qu'il n'y a pas de poussières visibles ou de saletés sur les fenêtres ou sur les tables. Si vous arrivez trop tard 
après le début de la journée et que vous ne pouvez pas juger si le linge était propre au départ, écrivez 98 dans l'encadré.

- "lumière suffisante" veut dire une lampe électrique fonctionnelle ou suffisamment de lumière naturelle à travers une fenêtre pour qu'une infirmière/sage-femme ou un médecin puisse faire un examen adéquat.

- "eau en quantité suffisante" veut dire de l'eau propre en quantité suffisante pour se laver les mains et les équipements. La source d'eau peut être un robinet ou un puits dans la concession.

16. Cochez si les types d'équipements suivants sont disponibles et fonctionnels dans l'unité de SMI/PF, et/ou dans la salle de stock pour les services SMI/PF.

17. Comptez le nombre de types d'équipements suivants disponibles et fonctionnels dans l'unité de SMI/PF et/ou dans la salle de stock pour les services SMI/PF.

C'est là l'une des sections les plus importantes. Chaque élément de la liste a été identifié comme nécessaire pour les services $\mathrm{PF}$, les services de SR, ou pour la prévention de l'infection. La présence ou l'absence d'un élément quelconque affectera énormément les services. Il faut vérifier la présence de chaque élément en voyant et en comptant tout l'équipement dans la clinique et dans la salle de stock. S'il n'est pas possible d'avoir accès à la salle de stock, écrivez le nombre vu dans la clinique et marquez ce problème à la marge. Il est très important de vérifier si l'équipement est fonctionnel. Par exemple, s'il y a une lampe torche mais qui n'a pas de piles, alors elle ne devrait pas être comptée.

S'il y a un stérilisateur mais qui n'est pas utilisé parce qu'il ne fonctionne pas, il ne devrait pas être compté. Si un équipement est partagé avec d'autres prestataires, il devrait être compté.
18. D'habitude l'unité de SMI/PF fournit-elle chacune des méthodes contraceptives suivantes?

19. (Si la méthode est fournie d'habitude) Vérifiez physiquement et cochez si les produits sont disponibles aujourd'hui.

20. (Si la méthode est fournie d'habitude) Y a$\mathrm{t}$-il eu une rupture de stock au cours des six derniers mois?

Le but de ces questions est de voir si le PPS fournit une méthode contraceptive particulière, et si oui, quelle est la fiabilité de ses stocks contraceptifs. Les stocks sont particulièrement importants parce que s'il n'existe pas de produits, un PPS ne peut pas fournir de méthode, même s'il est prêt à le faire.

Demandez au responsable des services PF les méthodes qui sont fournies en réalité dans le PPS. Cochez les encadrés correspondants de la question 18. Ensuite allez dans la salle de stock ou dans l'armoire et vérifiez la présence des provisions de contraceptifs. Cochez l'encadré qui se trouve en bas de la question 19 s'il existe au moins une unité non périmée de chaque type de produit. Rassurez-vous de bien vérifier la date de péremption. S'il n'y a pas une date de péremption, il ne faut pas compter l'élément en question. (Par exemple, il peut paraître bizarre de cocher l'encadré s'il n'existe qu'un seul cycle de pilules, mais après beaucoup d'expérimentation avec la méthodologie, nous avons constaté que le fait de compter le nombre de produits disponibles dans un PPS est extrêmement fastidieux et ne fournit pas davantage de données informatives.) S'il n'est pas possible d'accéder aux provisions, il faut donc écrire 98 dans ces encadrés.

L'information de la question 20 peut provenir de deux sources. D'abord, il faut demander au responsable des services PF ou au responsable de la logistique si certaines méthodes contraceptives ont connu une rupture de stock au cours des six derniers mois. Ensuite essayez de vérifier s'il y a eu 
rupture de stock en contrôlant les statistiques si elles sont disponibles. Si le responsable des services $\mathrm{PF}$ ou les statistiques indiquent une rupture de stock, cochez la colonne sous la question 20. S'il y a des doutes, écrivez 98 dans ces encadrés.

21. La structure sanitaire (y compris l'unité de SMI/PF) fournit-elle d'habitude les services suivants ou le counseling ?

Demandez au responsable des services PF si chaque service ou counseling est fourni d'habitude. "Fourni d'habitude" veut dire au moins trois jours par semaine.

22. Ce PPS fait-il des tests de laboratoire de MST, HIV, ou de grossesse ?

Les services de diagnostic des MST/VIH et de la grossesse devraient faire partie d'un programme complet de services PF/SR. Demandez au responsable des services PF si le PPS fait des tests de MST/VIH ou de grossesse. Si non, allez à la question 24 .

23. Y a-t-il des tests de (différentes MST/grossesse) disponibles dans cette unité de SMI/PF ou dans cette structure sanitaire, ou bien des prélèvements de clientes, ou les clientes elles-mêmes sontils envoyés ailleurs?

Le but de cette question est de savoir si ce genre de test est disponible pour les clientes qui fréquentent ce PPS, que le test soit fait sur site ou non. Demandez au responsable des services PF si les tests sont faits dans cette structure sanitaire ou si les clientes sont envoyées ailleurs, ou si des prélèvements sont envoyés hors du site pour être testés. $\mathrm{Si}$ le test n'est pas du tout offert, laissez les deux colonnes vides.

24. Cette structure sanitaire offre-t-elle d'habitude les services de vaccination suivants?

Les services de vaccination constituent une autre composante importante d'un programme intégré de PF/SMI. Demandez au responsable des services $\mathrm{PF}$ si chaque service de vaccination est fourni. "Fourni d'habitude" veut dire au moins trois jours par semaine.

25. (Si la vaccination est fournie) Les vaccins ont-ils connu une rupture de stock au cours des six derniers mois?

L'information de cette question peut provenir de deux sources. D'abord, il faut demander au responsable des services PF ou au responsable de la logistique si certains vaccins ont connu une rupture de stock au cours des six derniers mois. Ensuite essayez de vérifier s'il y a eu rupture de stock en vérifiant les statistiques si elles sont disponibles. Si le responsable des services PF ou les statistiques indiquent une rupture de stock, cochez la colonne sous la question 25 . S'il y a des doutes, écrivez 98 dans ces encadrés.

26. Existe-t-il un inventaire écrit des produits et des provisions suivants ?

27. (S'il existe un inventaire) Est-il à jour, lisible et complet ?

Demandez au responsable des services PF ou à celui de la logistique s'il existe un inventaire des produits énumérés. Un "inventaire" est un système permettant de garder les données sur le nombre de produits reçus et le nombre utilisé. S'il existe un inventaire, demandez à le voir et vérifiez s'il est à jour, lisible et complet. Si vous ne pouvez pas accéder à l'inventaire, écrivez 9 dans la colonne qui se trouve sous la question 27 .

28. Les produits suivants sont-ils stockés selon la date de péremption ? En d'autres termes, existe-t-il un système de "premier périmé premier sorti "? Un système de stockage basé sur la date de péremption protège les clientes contre les produits périmés et permet au PPS d'éviter 
les gaspillages. Pour chaque produit, demandez au responsable des services PF ou à celui de la logistique si les produits sont stockés selon la date de péremption. Vérifiez les réponses en demandant à voir par conséquent le système d'enregistrement des dates de péremption et de stockage.

29. Pour chaque produit, les lieux de stockage sont-ils protégés de la pluie, du soleil, des températures défavorables, des rats et des insectes nuisibles?

Si les produits sont exposés à ces différentes menaces, alors leur efficacité et leur innocuité seront compromises. Demandez à voir le lieu de stockage des produits et déterminez si les conditions de stockage sont satisfaisantes, c'est-à-dire pas exposés à la pluie ou au soleil, protégés contre les rats et les insectes nuisibles et pas sujets à une température trop élevée. Il faut noter que pour certains vaccins, la protection contre les températures défavorables impliquerait une réfrigération. Généralement, les produits sont stockés dans une salle séparée ou dans une armoire en acier ou en bois.

30. Comment les fiches des clientes sont-elles gardées dans cette structure sanitaire?

31. Quel est l'état du système des fiches de statistiques?

Ces questions traitent du problème de la continuité. Des fiches de données des clientes bien gardées permettent aux prestataires de se fonder sur les soins précédemment reçus et d'établir au moins partiellement l'antécédent médical de chaque cliente. Demandez au responsable des services $\mathrm{PF}$ le système d'utilisation des fiches de clientes : les fiches sont-elles gardées dans le PPS ou par les clientes ? Si elles sont gardées dans le PPS, demandez à les voir. Usez de votre jugement personnel pour déterminer si le système est bien ordonné, partiellement ordonné mais encore utilisable, ou pas utilisable.
32. Existe-t-il un seul registre des activités quotidiennes pour tous les services SMI/PF fournis par la structure sanitaire?

Les registres des activités quotidiennes sont utiles pour le responsable du PPS pour surveiller le volume de la clientèle et planifier les services à venir. Demandez s'il existe un registre des activités quotidiennes pour tous les services. Vérifiez l'existence des registres en demandant à les voir.

33. Les rapports de statistiques des services suivants sont-ils envoyés à un superviseur ou à une unité supérieure ?

Il est important que les statistiques des services soient envoyées à une unité supérieure parce que cela permet une supervision et une coordination centrale des services, ce qui peut améliorer leur qualité. Pour chaque service, demandez au responsable des services PF si des rapports mensuels des statistiques du volume de la clientèle sont envoyés à un superviseur ou à une unité supérieure.

34. Au cours des six derniers mois, combien de fois un superviseur est-il venu dans cette unité de SMI/PF pour des raisons de supervision?

Généralement, les gens ont tendance à faire mieux s'ils savent que quelqu'un est intéressé par leur travail et s'en préoccupe. Les visites de supervision devraient servir à cette fin et de ce fait améliorer la qualité des services. Demandez au responsable des services PF le nombre de visites de supervision qui ont été faites au cours des six derniers mois dans le but de revue des services. Il est important que vous déterminiez que les visites ont été faites pour des buts de supervision, et non pour fournir des services ou pour une autre raison. S'il existe un registre de visites de supervision, demandez à le consulter. 
35. En visitant cette structure sanitaire, que fait le superviseur?

S'il y a eu une visite de supervision au cours des six derniers mois, demandez au responsable des services $P F$ ce que le superviseur a fait.

\section{Combien de clientes ont reçu les services} suivants au cours des 12 derniers mois?

37. Indiquer le dernier mois et l'année rapportés dans le tableau ci-dessus. Le but de ces questions est de mesurer le volume de la clientèle, par service, dans chaque PPS. Ceci peut aider à illustrer la corrélation entre la qualité des services et l'utilisation des services.

Demandez à voir les statistiques de services du PPS. Pour chaque service, vérifiez si le PPS dispose de 12 mois consécutifs de statistiques de services au cours des 24 derniers mois. Vous pouvez utiliser n'importe quels mois dans la période des 24 mois, pourvu que les 12 mois soient consécutifs. S'il y a des vides et qu'il est impossible d'obtenir 12 mois consécutifs, prenez donc le groupe de mois les plus récents pour lesquels les statistiques sont disponibles. Dans certains cas, seul deux ou trois mois consécutifs peuvent être possibles.

Dans les colonnes A et B, enregistrez le nombre total de nouvelles et d'anciennes clientes pour chaque service. Si vous avez des problèmes à lire les statistiques, faites une note à la marge. Entrez le code "99999" si les statistiques ne sont pas disponibles. Ecrivez le nombre de mois que vous utilisez (1 à 12) dans la colonne $C$.

Une fois le tableau rempli, écrivez dans la question 37 le mois et l'année du mois le plus récent utilisé dans le tableau. Ceci donne une idée du caractère récent des données.
38. Quel est le coût de chaque méthode ou service pour la cliente? (Frais de consultation, produit/test/procédure) L'accessibilité à une méthode ou à un service est affecté par son coût. En recueillant les informations sur les frais que les clientes payent, nous pouvons mieux comprendre les barrières financières au service. Demandez au responsable des services $\mathrm{PF}$ les frais que les nouvelles clientes payent pour chaque méthode ou service. Rappelez-vous qu'il s'agit là des frais que les clientes payent en réalité, et non pas le coût payé au PPS pour la fourniture d'une méthode ou d'un service.

Demandez les frais payés par une nouvelle cliente moyenne, pas une qui a des complications avec la méthode ou le service ou qui a un antécédent médical peu courant. Assurez-vous de diviser les frais entre la consultation (colonne A) et le produit, le test, et la procédure (colonne B). Si la méthode/service a des frais à la fois pour le produit et la procédure, comme c'est le cas de la pose du DIU, enregistrez dans la colonne B le total des frais payés par la cliente pour tous les produits, tests et procédures combinés. Pour certaines méthodes/services tels que le counseling en MAMA ou le counseling en nutrition, il n'y aura pas de frais pour le produit/test/procédure. Si la méthode ou le service n'est pas offert dans le PPS, il faut laisser les deux colonnes vides. 


\section{Guide d'observation}

Cet instrument de collecte des données est conçu pour vous aider à enregistrer ce qui se passe lorsqu'un prestataire tel qu'une infirmière/sagefemme ou un médecin, conseille et consulte une cliente de planification familiale. Cette observation est l'une des parties les plus importantes d'une étude d'Analyse Situationnelle. Elle est peut-être aussi la plus difficile à remplir parce qu'elle nécessite de bonnes dispositions d'écoute et d'observation. Souvent, en observant l'interaction entre la cliente et le prestataire, vous aurez à prendre des notes et à enregistrer plus tard la réponse à certains éléments. Vous devriez essayer de vous familiariser bien avec le guide d'observation de manière à savoir ce que vous recherchez pendant l'interaction entre la cliente et le prestataire et à savoir aussi où vous devez enregistrer l'information. La section introductive de l'Observation : guide question par question (page 57) aussi bien que la section "Astuces pour réaliser une bonne observation" (page 23) donnent davantage d'information sur la manière de remplir cet instrument.

Les codes présentés entre accolades \{\} dans cet instrument sont des noms de variables proposés pour chaque question ou marque à cocher. On se réfère à ces noms dans le plan d'analyse (commençant à la page 166). Cependant, il faut garder à l'esprit qu'ils n'apparaîtront pas dans le questionnaire utilisé par les chercheurs sur le terrain. Voir les pages qui suivent pour l'instrument. 


\section{Guide d'observation de l'interaction entre \\ les clientes de planification familiale et les prestataires de services}

INSTRUCTIONS A L'ATTENTION DE L'OBSERVATEUR : II faut obtenir le consentement de la cliente et du prestataire avant de procéder à l'observation de l'interaction entre eux. Assurez-vous que le prestataire sait que vous n'êtes pas là pour l'évaluer et que vous n'êtes pas un "expert" qu'il peut consulter pendant la session. En observant, soyez aussi discret que possible : Asseyez-vous de sorte que vous vous trouviez derrière la cliente mais pas directement face à face avec le prestataire, et prenez vos notes rapidement. Pour chaque question, encerclez la réponse qui représente le mieux votre observation de ce qui s'est passé pendant l'interaction. Comme discuté pendant la formation, vous pouvez être témoins de comportements qui constituent de sérieuses menaces à la santé de la cliente. Veuillez garder en tête les directives relatives au moment où vous devez intervenir dans la consultation pour le bien-être de la cliente.

Structure sanitaire visitée (nom) : \{NOMFS\}

Code de la structure sanitaire : \{CODEFS\}

District (nom) : $\{$ NOMDIST\}

Code du district : \{CODEDIST\}

Village/ville (nom) : \{NOMVILLE\}

Code Village/ville : \{CODEVILLE\}

Numéro d'étude de la cliente \{IDCL $\}$

Lieu de la cliente/salle $\{$ LCL $\}$

Numéro d'étude de l'agent de santé \{IDAS\}

Date de la visite : Jour Mois Année \{DATE\}

Nom de l'observateur :

Signature du chef d'équipe : 
Type de la structure sanitaire: \{TYPE\}

1 = Hôpital de référence

2 = Hôpital de district

$3=$ Hôpital primaire

4 = Centre de santé rural

5 = Maternité

6 = Poste de santé

$7=$ Pharmacie

$8=$ DBC

$9=$ Autre
Type de secteur: $\{$ SECT $\}$

$1=$ Gouvernement

2 = APF

$3=$ Mission

4 = Privé

5 = Autre

Localité: $\{\mathrm{LOC}\}$

1 = Rurale

2 = Urbaine

Désignation de l'agent de santé: $\{$ DESIG\}

1 = Médecin

2 = Infirmière

3 = Infirmière/sage-femme

$4=$ DBC

$5=$

$6=$

7 = Deux prestataires ou plus :

Spécifier :

$\overline{\{D E S I G 1\}} \overline{\{D E S I G 2\}} \overline{\{D E S I G 3\}}$

Début de l'observation :

\{DEBUTOB\}

But principal de la visite comme indiqué premièrement par la cliente : \{BUT\}

Nouvelles clientes:

1 = Nouvelle utilisatrice de PF

2 = utilisatrice de PF "Recommençant"

Anciennes clientes :

3 = Visite de réapprovisionnement ou visite de suivi (sans problèmes)

$4=$ Visite de réapprovisionnement ou visite de suivi (problème avec la méthode, veut changer de méthode, ou veut abandonner l'utilisation de la PF) 


\section{Salutations et examens de la cliente}

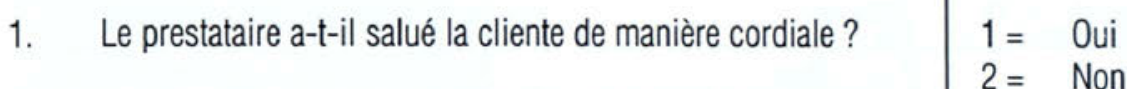

2. Le prestataire a-t-il posé des questions sur l'un des sujets Suivants ou ont-ils été abordés

spontanément par la cliente?

\{02X1\} $1=$ Si la cliente veut davantage d'enfants dans le futur

\{02X2\} $2=$ Age de l'enfant le plus jeune

\{02X3\}

(Encerclez tout ce qui s'applique)

$3=$ Si la cliente allaite

(Cochez si la cliente allaite pendant l'observation :

\{02X4\} $\quad 4=$ Statut matrimonial de la cliente

$\{02 \times 5\} \quad 5=\quad$ Si la cliente a eu plus d'un partenaire sexuel au cours des années passées.

$\{02 \times 6\} \quad 6=$ Si la cliente a des questions

102X7\} $\quad 7=$ Si la cliente s'inquiète de sa propre santé

\{02X8\} $8=$ Si la cliente a des inquiétudes par rapport à l'utilisation d'une méthode

\{02X9\} $9=$ Si la cliente a des inquiétudes par rapport aux MST ou au $\mathrm{VIH} / \mathrm{SIDA}$

\{02X10 $\} \quad 10=$ Si la cliente a des symptômes/signes/traitements antérieurs qui rappellent des MST

\{02X11\} $11=$ Si la cliente a discuté de planification familiale avec son mari/partenaire

102X77\} $77=$ Aucun de ces sujets

3 Le prestataire a-t-il posé des questions sur l'un des sujets suivants ou ont-il été abordés spontanément par la cliente?

\{03X1\} 1 = Antécédent médical/familial

\{03X2\} $2=$ Date de la LMP

\{03X3\} $3=$ Saignement vaginal anormal

\{03X4 $\quad 4=$ Ecoulement vaginal anormal

(Encerclez tout ce qui s'applique)

$\{03 \times 5\} \quad 5=$ Démangeaison de l'appareil génital

\{03X6\} $6=$ Douleur au bas ventre

103X77\} $77=$ Aucun de ces sujets

4. Pendant la consultation, le prestataire a-t-il fait l'une des actions suivantes?

$1=$ Contrôle du poids (vérifiez la carte)

\{04X2\} $2=$ Prise de la tension artérielle (vérifiez la carte)

[04X3\} $\quad 3=$ Test de grossesse /référer pour test de grossesse

(Encerclez tout ce qui s'applique)

$4=$ Examen physique général

\{04X5\} $\quad 5=$ Test sanguin fait/demandé/référer pour test sanguin

(04X6) $6=$ Examen mammaire

$7=$ Test de Papanicolaou fait/demandé/référée pour test de

$\{04 \times 7\}$ Papanicolaou (voir procédures médicales faites)

\{04X77\}

$8=\quad$ Analyse symptomatique pour MST

$77=$ Aucune de ces actions 


\section{Discussion sur la planificacion familiale}

5. Le prestataire a-t-il posé des questions sur les méthodes précédemment utilisées ou ont-elles été mentionnées spontanément par la cliente?

6. Avant de venir dans cette structure sanitaire, quelle est la méthode contraceptive que la cliente utilisait? \{06\}

7. Le prestataire a-t-il demandé à la cliente si elle voulait changer de méthodes ou abandonner leur utilisation, ou la cliente a-t-elle mentionné ce désir spontanément?

8. Le prestataire a-t-il demandé à la cliente les problèmes qu'elle éprouve avec la méthode actuelle ou la cliente les a-t-elle mentionnés spontanément?

9. (Si la cliente avait des problèmes avec sa méthode)

Le prestataire a-t-il fait

l'une des actions suivantes?

(Encerclez tout ce qui s’applique.)

\{09X1\}

\{09X2\}

109X3\}

\{09X4\}

$\{09 \times 77\}$

10. Le prestataire a-t-il demandé ou la cliente a-t-elle mentionné spontanément une préférence pour une méthode contraceptive spécifique?
$1=$ Oui

$2=$ Non
Code de la Méthode :

$1=0$ Oui

$2=$ Non

$1=$ Oui

$2=$ Non

$1=\quad$ A conseillé la cliente sur le problème

$2=\quad$ A fait un traitement médical

$3=\quad$ A suggéré/consenti que la cliente change de méthode

$4=\quad$ A référé la cliente ailleurs pour traitement

$77=$ Aucune action
$1=$ Oui

$2=$ Non

$\{010\}$

11. (Si oui) Quelle méthode? $\{011\}$

Code de la méthode:

12. Veuillez noter les méthodes que le prestataire a mentionnées ou discutées pendant la consultation.

(Encerclez tout ce qui s'applique.)

$\{012 \times 1\}$ à $\{012 \times 13\}$

13. (Si la cliente accepte une méthode ou change de méthode). Par rapport à la nouvelle méthode acceptée, le prestataire a-t-il parlé de l'un des sujets suivants?

(Encerclez tout ce qui s'applique.)
$\{013 \times 1\}$

$\{013 \times 2\}$

\{013×3\}

\{013X4\}

$\{013 \times 5\}$

$\{013 \times 6\}$

$\{013 \times 7\}$

\{013X77\}
Codes des méthodes:

$\begin{array}{lllll}1 & 2 & 3 & 4 & 5 \\ 6 & 7 & 8 & 9 & 10 \\ 11 & 12 & 13 & & \end{array}$

$1=$ Comment utiliser la méthode

$2=$ Avantages

$3=$ Inconvénients

$4=\quad$ Effets secondaires Médicaux (ex. saignement, nausée, etc.)

$5=\quad$ Ce que la cliente doit faire en cas de problème avec la méthode

$6=$ Possibilité de démangeaisons

7 = Capacité de la méthode à prévenir les MST/NIH

$77=$ Aucun de ces sujets 
14. (Si la cliente choisit la MAMA)

Le prestataire a-t-il mentionné les points suivants?

(Encerclez tout ce qui s'applique)
\{014X1\}

$\{014 \times 2\}$

\{014X31 $\quad 3=$ La cliente doit allaiter exclusivement/presque exclusivement

\{014X77\} $77=$ Aucun de ces points

15. Le prestataire a-t-il donné trop d'importance à une méthode particulière? \{015\}

16. Quels matériels IEC, le cas échéant, ont été utilisés pendant la consultation?

\{016x1\} 1 = Boîte à image

\{016×2\} 2 = Dépliants/brochures

(Encerclez tout ce qui s'applique)

(016)3\} $\quad 3=$ Echantillons contraceptifs

\{016 $\times 4\} \quad 4=$ Affiches

(016 $\times 5\} \quad 5=$ Autre :

\{016 $\times 77\} \quad 77=$ Aucun

\section{Discussion sur les MST et autres questions de santé}

17. Pendant la consultation, le prestataire a-t-il explicitement mentionné que le préservatif protège contre les MST/NIH/SIDA ? [017\}

18. Le prestataire a-t-il indiqué à la cliente qu'il se peut qu'elle ait une MST ? (018)

19. (Si oui) Qu'est-ce que le prestataire a fait ?

(Encerclez tout ce qui s'applique)

20. Quels sont les autres sujets de santé qui ont été mentionnés à n'importe quel moment de la consultation?

(Encerclez tout ce qui s'applique)
$1=$ Oui

$2=$ Non

$1=0$ Oui

$2=$ Non

$1=$ Demande des tests de laboratoire

\{019×2\} $2=$ Traite

\{019X3\} $3=$ Réfère ailleurs

\{019X4\} $\quad 4=$ Donne des conseils

[019X5\} $5=$ Autre:

\{019X5R\}

\{019X77\} $77=$ Aucune action prise

\{020X1\} $1=\mathrm{VIH} / \mathrm{SIDA}$

\{020X2\} $2=$ Autres MST

\{020X3\} $3=$ Vaccination des enfants

\{020X4 $\quad 4=$ Surveillance de la croissance des enfants

\{020X5\} $5=$ Infécondité

1020X61 $6=$ Thérapie de réhydratation orale

1020X7\} $7=$ Avortement

1020X81 $8=$ Nutrition

1020X9\} $9=$ Services curatifs - cliente

$\{020 \times 10\} \quad 10=$ Services curatifs - enfant

\{020X11\} $11=$ Allaitement

1020X12\} $12=$ Rapports sexuels

\{020 $\times 13\} \quad 13=$ Facteurs socio/économiques

[020X14] $14=$ Test de grossesse

1020X15\} $15=$ Autre:

[020X15R\}

$77=$ Aucun de ces sujets 


\section{Décision relative à la planificacion familiale, à l'approvisionnement, et au suivi}

21. La cliente a-t-elle décidé d'utiliser une méthode contraceptive? $\{021\}$

22. (Si non) Quelle est la principale raison pour laquelle la cliente n'a pas choisi de méthode? $\quad$ 1022\}

(Encerclez une.)

(Pas d'autres questions dans cette section.)

$1=$ Oui

$2=$ Non

$1=\quad$ Est venue s'informer seulement

$2=\quad$ A changé d'avis

$3=$ Grossesse suspectée

$4=$ Contre indications médicales

$5=$ Allaitement

$6=$ Autres raisons de santé

$7=\quad$ Méthode non disponible

$8=$ Aucune raison apparente

$9=$ Autre

23. Quelle décision la cliente a-t-elle prise par rapport à la planification familiale?

(Encerclez une.)

24. (Si arrête ou change) Quelle est la raison principale d'arrêter ou de changer?

(Encerclez une.)

(Si arrête, pas d'autres questions dans cette section.)

$1=\quad$ Continuer avec la même méthode

$2=$ Arrêter

$3=\quad$ Changer

$1=\quad$ Veut une grossesse

$2=$ Effets secondaires non désirés

$3=$ Approvisionnement non convenable

$4=\quad$ Mari/partenaire n'aime pas la méthode

$5=\quad$ Change de méthode d'espacement pour méthode permanente

$6=$ Autre

$8=$ Raison non claire

25. Pour quelle méthode la cliente a-t-elle décdé/opté?

26. La méthode que la cliente a décidé d'utiliser est-elle la même qu'elle a préférée au départ ? (Voir q.10 et 11)

Code de la méthode:

$1=$ Oui

$2=$ Non

$3=\quad$ Pas de préférence exprimée

27. (Si non) Pourquoi la cliente a-t-elle décidé de ne pas utiliser la méthode qu'elle avait préférée initialement?

(Encerclez une.)

1 = La cliente a changé d'avis après avoir entendu parler des méthodes

$2=$ Le prestataire a pensé qu'il y avait des contre indications médicales

$3=$ Le prestataire a pensé que la méthode n'était pas appropriée à cause de l'âge, du statut matrimonial...

$4=$ Grossesse suspectée

$5=$ Méthode en rupture de stock

$5=$ Autre :

$7=\quad$ Pas de raison apparente 


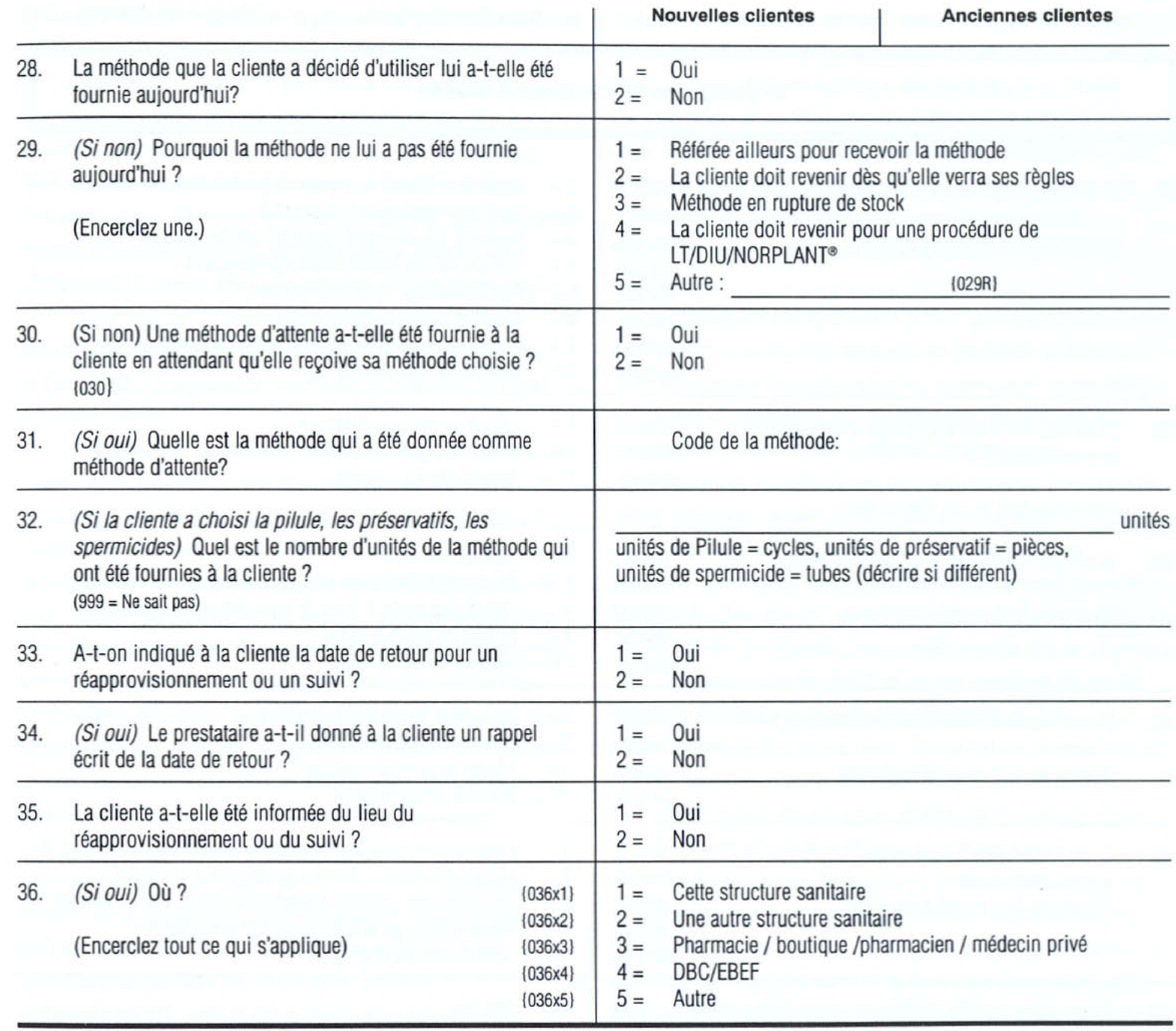




\section{Procédures Médicales réalisées}

37. Si un examen pelvien a été fait, le prestataire a-t-il $\quad\left\{037 x_{1}\right\} \quad 1=\quad$ Informé la cliente de ce qui se produirait avant l'examen ?

\{037X2\} $2=$ Lavé les mains avant l'examen?

(Encerclez tout ce qui s'applique)

\{037X3\} $\quad 3=$ Inspecté visuellement l'appareil génital externe?

\{037X4\} $\quad 4=$ Fait un test de Papanicolaou/prélèvement ?

\{037X5\} $\quad 5=$ Fait un examen manuel/bimanuel ?

$\{037 \times 6\} \quad 6=$ Lavé les mains après l'examen ?

1037X7] $\quad 7=$ Informé la cliente du résultat après l'examen?

[037X77\} $77=$ Aucune de ces actions

38. Si un examen à été fait à l'aide d'un spéculum : $\quad$ \{038X1\} $\quad 1=\quad$ Utilisé un spéculum propre ?

le prestataire a-t-il

1038X2) $2=$ Utilisé des gants ? (voir la question sur les gants)

[038X77\} $77=$ Aucune de ces actions

(Encerclez tout ce qui s'applique)

39. Si un DIU a été posé le prestataire a-t-il

$\{039 \times 1\}$

\{039X2\}

$1=$ Sondé l'utérus ?

(Encerclez tout ce qui s'applique)

\{039X3\} $\quad 3=$ Utilisé des gants ? (voir la question sur les gants)

\{039X4\} $4=$ Donné un soutien moral ?

\{039X77\} $77=$ Aucune de ces actions

40. Si une injection a été faite, le prestataire a-t-il

\{040X1\} $1=$ Désinfecté le point d'injection?

\{040X2\} $2=$ Utilisé une aiguille stérile ?

(Encerclez tout ce qui s'applique)

\{040X3\} $3=\quad$ Massé le point d'injection?

\{040X77\} $77=$ Aucune de ces actions

41. Si un NORPLANT ${ }^{\circledR}$ a été posé,

\{041X1\} 1 = Lavé le bras de la cliente avec du savon et de l'eau ?

le prestataire a-t-il

(Encerclez tout ce qui s'applique)

\{041X2\} $2=\quad$ Utilisé des gants ? (voir la question sur les gants)

[041X31 $\quad 3=$ Appliqué une solution antiseptique sur le site d'insertion?

\{041X4\} $\quad 4=$ Placé le bras de la cliente sur un tissu stérile ?

\{041X77\} $77=$ Aucune de ces actions

42. Si des gants ont été utilisés, les gants étaient-ils

\{042\}

$1=$ Stériles

$2=$ Propres mais non stériles

(Encerclez tout ce qui s'applique)

$3=$ Pas propres

98 = Incapable de déterminer le niveau de propreté

Fin de l'observation : $\{\mathrm{FINOB}\}$

Durée de l'interaction:

$\{\mathrm{DUR}\}$

Agrafez une interview de sortie à ce questionnaire, suivez la cliente hors de la salle de consultation, présentez-la, et donnez les deux questionnaires à l'interviewer.

Codes des méthodes:

1 Pilule combinée (ou non spécifiée)

8 Spermicide

2 Pilule progestative

9 Ligature des trompes (ML/AL)

3 DIU

4 Injectable

10 Vasectomie

5 NORPLANT $^{\circ}$

11 PFN

6 Préservatif

12 MAMA

7 Diaphragme

13 Autre 


\section{Observation : guide question par question}

Prenez bonne note des instructions adressées à l'observateur à la première page de cet instrument. Toutes les instructions sont importantes. Vous devriez pouvoir remplir la plupart des données des deux premières pages avant le début de l'observation. Si vous avez des doutes sur l'un des codes d'identification, demandez à votre Chef d'équipe. Ecrivez (ne pas signer) votre nom dans l'encadré qui se trouve au bas de la deuxième page. Lorsque vous aurez terminé l'observation, votre Chef d'équipe la révisera et signera en bas de votre nom.

Vers la fin de la seconde page, vous trouverez aussi "But principal de la visite comme indiqué prioritairement par la cliente:" Veuillez garder à l'esprit que nous avons des attentes différentes de la part d'un prestataire qui fournit des services à une "nouvelle cliente" et à une "ancienne cliente." Ces deux types de clientes peuvent être classées davantage comme l'indique les codes. Pour les nouvelles clientes, soyez très attentif à toutes les questions ou à toute mention faite de l'utilisation précédente ou non d'une méthode contraceptive par la cliente. Si elle n'a jamais utilisé une méthode contraceptive jusqu'ici, elle est une "nouvelle" acceptante de PF. Si elle a utilisé au moins une méthode dans le passé, mais n'utilise pas actuellement une méthode, elle est une "recommençante." Si la cliente utilise présentement une méthode (juste avant cette visite) elle est une "ancienne cliente." Sachez qu'une ancienne cliente peut venir au PPS pour une multitude de raisons. Il y a un véritable chevauchement entre "problème avec la méthode," "voulait changer de méthode" et "voulait abandonner la PF," parce que toutes ces situations sont généralement liées aux clientes qui éprouvent des problèmes. De ce fait, nous ne tentons pas de faire une distinction entre ces différentes clientes.

Au fur et à mesure que vous avancez dans l'instrument, gardez à l'esprit qu'une observation est différente d'une interview en ce sens que nous ne savons pas l'ordre dans lequel les éléments d'intérêt seront soulevés au cours de l'interaction. Il n'y a pas un ordre établi comme c'est le cas des questions de l'interview. Vous devez vous familiariser avec tous les éléments que vous comptez observer et leur emplacement dans la fiche de collecte des données, de sorte que vous pouvez retrouver rapidement un élément et le coder pendant que l'interaction continue.

Plusieurs rubriques d'ordre général organisent en quelque sorte les questions et vous aideront à vite retrouver l'emplacement approprié pour coder un point d'intérêt. Ces rubriques comprennent :

Salutations et examen de la cliente, qui couvre l'information médicale et sociale que le prestataire peut chercher à savoir afin de mieux examiner la cliente pour la PF; Discussion sur la planification familiale, qui inclut des informations spécifiques sur les méthodes de $\mathrm{PF}$, les antécédents de la pratique de la PF de la cliente, les préférences de la cliente, etc.; Discussion sur les MST et autres questions de santé; Décision relative à la planification familiale, à l'approvisionnement, et au suivi. Pendant la formation et la pratique, vous devez vous familiariser avec l'emplacement de chaque élément d'intérêt dans l'instrument d'observation.

Notez que ce formulaire a deux colonnes sur le côté droit-une pour les nouvelles clientes et une pour les anciennes clientes. La plupart des questions s'appliquent à la fois aux nouvelles et aux anciennes clientes, mais d'autres questions ne s'appliquent qu'à un seul type de clientes. Lorsqu'une question ne s'applique qu'à une nouvelle cliente, la colonne de l'ancienne cliente sera grisée, et vice versa. Lorsqu'une question s'applique à la fois aux nouvelles et aux anciennes clientes, la ligne séparatrice entre les colonnes disparaît.

Pour plusieurs questions, au lieu d'écrire le nom de chaque méthode de PF, on vous demandera d'utiliser un code pour chaque méthode. La liste des codes des méthodes se trouve sur la dernière page de l'instrument d'observation (page 56). Cette liste des codes est utilisée chaque fois que vous devez enregistrer les 
méthodes contraceptives dans l'observation, et avec le temps il vous serait peut-être plus facile d'utiliser les codes plutôt que d'écrire le nom de chaque méthode.

Après l'observation, suivez la cliente hors de la salle et demandez-lui poliment si elle aimerait être interviewée sur son expérience. Si elle consent, présentez-la donc à l'enquêteur, spécialiste des sciences sociales.

La révision de l'instrument à la fin de l'observation est une étape extrêmement importante. Consultez tout l'instrument et voyez si chaque élément d'intérêt a eu lieu. Vous devriez être particulièrement attentif au code 77 qui indique qu'aucun des sujets n'a été discuté/ observé. Vous aurez aussi besoin de parcourir la colonne "nouvelle" ou "ancienne" du côté droit de la page pour vérifier que l'observation et l'enregistrement sont complets, et que vous avez toujours codé le matériel dans la bonne colonne.

Le formulaire d'observation est plus ou moins condensé; une grande somme d'information doit être enregistrée sur quelques pages. La raison pour laquelle cet instrument est condensé et utilise certains "raccourcis" (codes des méthodes) est de réduire le nombre de feuilles qu'il faut retourner pour trouver un élément particulier. Une version précédente et beaucoup plus longue de l'observation a conduit à des erreurs lorsque les observateurs ne pouvaient pas retrouver rapidement les éléments dont ils avaient besoin.

Bien que généralement nous ne voulons pas perturber l'interaction prestataire-cliente, et en le faisant cela pourrait invalider les objectifs de recherche de la visite, il faut toutefois vous rappeler les directives données pendant la formation par rapport à ce que vous devez faire lorsque vous constatez que le comportement d'un prestataire constitue un sérieux risque pour la santé de la cliente. Veuillez garder à l'esprit les directives relatives au moment d'intervenir dans la consultation pour le bien-être de la cliente. (Voire la section relative aux "Problèmes d'ordre éthique" pour plus d'informations, page 16)

\section{Salutations et examen de la cliente}

\section{Le prestataire a-t-il salué la cliente de} manière cordiale ?

Référez-vous à votre formation pour une définition culturelle appropriée. Rappelez-vous de bien faire attention au ton, aux expressions faciales, aux gestes, au contact des yeux, à tout ce qui peut être utilisé pour déterminer si la salutation était cordiale ou non.

2. Le prestataire a-t-il posé des questions sur l'un des sujets suivants ou ont-ils été abordés spontanément par la cliente ? Nous voulons savoir si le prestataire possède l'information. Nous ne faisons pas de différence entre le fait que la cliente ait mentionné un point spontanément ou posé une question sur un élément. Sachez que si la cliente allaite pendant la prestation de services, il se pourrait qu'elle ne mentionne pas l'allaitement, et que normalement on ne lui poserait pas la question de savoir si elle est allaitante. Il y a un endroit spécial à cocher si la cliente allaite pendant la prestation de services. Rappelez-vous que ces éléments ne peuvent être notés que lorsqu'ils se produisent ou bien à la fin de l'observation au moment où vous révisez l'observation.

3. Le prestataire a-t-il posé des questions sur l'un des sujets suivants ou ont-ils été abordés spontanément par la cliente ?

4. Pendant la consultation, le prestataire a-til fait l'une des actions suivantes?

Nous voulons savoir si vous avez observé une de ces actions pendant l'interaction. La question traite des problèmes de santé avec la cliente, et la question 4 porte sur les évaluations physiques de la cliente qui ont été faites. Remarquez l'utilisation du code 77 dans les deux questions, indiquant qu'aucun de ces éléments n'a été discuté ou réalisé. Ce code ne peut être utilisé que pendant votre révision finale, lorsque la consultation est terminée. 
Il est possible que certaines de ces actions-telles que la prise de la tension artérielle-aient été faites dans un autre endroit de la structure sanitaire par un autre prestataire qui a marqué l'information dans la fiche de données de la cliente. Si possible, vérifiez la fiche de données de la cliente pour de telles actions, en plus des observations de l'interaction spécifique cliente-prestataire que vous êtes en train de faire.

\section{Discussion sur la planification familiale}

5. Le prestataire a-t-il posé des questions sur les méthodes précédemment utilisées ou ont-elles été mentionnées spontanément par la cliente?

Cette information n'est recherchée que pour les nouvelles clientes.

6. Avant de venir dans cette structure sanitaire, quelle est la méthode contraceptive que la cliente utilisait ? Sachez que la cliente ne serait en train d'utiliser une méthode avant de venir dans cette structure sanitaire que lorsqu'elle est une ancienne cliente. L'emplacement pour la réponse de "nouvelles clientes" est mis en grisé. Utilisez les codes des méthodes à la page 56.

7. Le prestataire a-t-il demandé à la cliente si elle voulait changer de méthodes ou abandonner leur utilisation, ou la cliente a-t-elle mentionné ce désir spontanément? Sachez que ceci implique que la cliente utilisait déjà une méthode. De ce fait, cette question ne devrait être posée qu'aux anciennes clientes.

8. Le prestataire a-t-il demandé à la cliente les problèmes qu'elle éprouve avec la méthode actuelle ou la cliente les a-t-elle mentionnés spontanément?

9. (Si la cliente avait des problèmes avec sa méthode) Le prestataire a-t-il fait l'une des actions suivantes?

Ces questions ne doivent être remplies que pour les anciennes clientes. Notez que la question 9 est en retrait et commence par " $(\mathrm{Si}$ la cliente avait des problèmes avec sa méthode)." Ne passez à la question 9 que lorsque la réponse de la question 8 est "oui." Remarquez le code 77 de la question 9, qui doit être utilisé si la cliente avait des problèmes mais que le prestataire n'a fait aucune action.

10. Le prestataire a-t-il demandé ou la cliente a-t-elle mentionné spontanément une préférence pour une méthode contraceptive spécifique préférée?

\section{1. (Si oui) Quelle méthode?}

Les recherches montrent que beaucoup de clientes ont une méthode contraceptive en tête lorsqu'elles viennent au PPS et qu'elles ont tendance à être plus heureuses et continueront à utiliser leur méthode pendant plus longtemps lorsqu'elles obtiennent la méthode qu'elles avaient en tête au début. Bien sûr, le prestataire peut découvrir des contre-indications médicales ou d'autres raisons pour lesquelles la cliente ne peut pas obtenir la méthode qu'elle avait en tête, mais nous voulons savoir si elle avait une préférence dans tous les cas. Nous sommes particulièrement intéressés par cette information avant de faire l'éducation ou le counseling lorsque l'information relative à la méthode spécifique est donnée. Notez l'exemple du saut-ne répondez à la question 11 que lorsque la réponse de la question 10 est "oui." Utilisez les codes des méthodes de la page 56.

12. Veuillez noter les méthodes que le prestataire a mentionnées ou discutées pendant la consultation.

Sachez que toutes les méthodes ne doivent pas être mentionnées à la fois. Chaque fois que le prestataire mentionne ou discute une 
méthode, il faut la cocher en conséquence. Toute mention faite d'une méthode quelle que soit la méthode est prise entièrement en compte. Utilisez les codes des méthodes de la page 56.

13. (Si la cliente accepte une méthode ou change de méthode). Par rapport à la nouvelle méthode acceptée, le prestataire a-t-il parlé de l'un des sujets suivants ? Cette question enregistre le contenu de l'information donnée à la cliente sur la méthode qu'elle a acceptée. Les clientes qui ont reçu davantage d'information sur leur méthode, en particulier sur les effets secondaires, ont tendance à utiliser la contraception pendant plus longtemps. Notez le code 77 si aucun de ces problèmes n'a été discuté.

14. ( $\mathrm{Si}$ la cliente choisit la MAMA) Le prestataire a-t-il mentionné les points suivants?

MAMA signifie méthode d'allaitement maternelle et d'aménorrhée. Il est peu probable que le prestataire utilise ce terme; soyez attentif à une description de l'allaitement exclusif comme méthode de PF et cochez en conséquence. N'utilisez le code 77 que lorsque la cliente a choisi la MAMA mais que le prestataire n'a mentionné aucune dimension importante de la MAMA.

15. Le prestataire a-t-il donné trop d'importance à une méthode particulière ? Trop d'importance donnée peut être indiquée par divers comportements des prestatairesle fait de discuter d'une seule méthode de PF, de ne mentionner que les aspects positifs d'une méthode et que les aspects négatifs des autres, ou le fait d'être très enthousiaste pour une méthode et peu enthousiaste pour les autres. Nous recherchons ici des exemples où les prestataires démontrent des biais en faveur d'une méthode particulière. Une recommandation spécifique de la part du prestataire pour cause d'une condition médicale ou de circonstances particulières qui se produisent pendant le counseling peut constituer ou non une "emphase."

16. Quels matériels d'IEC, le cas échéant, ont été utilisés pendant la consultation? Certains prestataires utilisent des matériels visuels pour aider à démontrer les arguments qu'ils ont avancés. Ces matériels peuvent inclure des affiches, des dépliants, des boîtes à images ou des échantillons de contraceptifs mêmes. Par exemple, il est courant que les prestataires tiennent une plaquette de pilules et montrent à la cliente la manière de commencer à les prendre ainsi que l'ordre dans lequel il faut les prendre. Souvent, l'utilisation de ces matériels d'IEC transmettent l'information aux clientes mieux que la parole seule.

L'instrument de l'inventaire enregistre le stock des matériels d'IEC qui sont disponibles dans chaque PPS. Cette information sera utilisée dans l'analyse des données pour déterminer si des matériels d'IEC étaient disponibles pour chaque prestataire. Si le prestataire que vous observez n'utilise pas de matériels d'IEC parce qu'ils ne sont pas disponibles dans le PPS, utilisez le code 77 . Le fait que le prestataire ne disposait pas de matériels d'IEC sera traité plus tard.

\section{Discussion sur les MST et autres questions de santé}

17. Pendant la consultation, le prestataire a-t-il explicitement mentionné que le préservatif protège contre les MST/NIH/SIDA ?

Les préservatifs sont utilisés à la fois pour la PF et la prévention des maladies. Nous voulons savoir si la fonction de prévention des MST des préservatifs a été mentionnée explicitement, même si la cliente n'utilise pas 
présentement les préservatifs, ou ne prend pas les préservatifs comme sa méthode aujourd'hui.

18. Le prestataire a-t-il indiqué à la cliente qu'il se peut qu'elle ait une MST ?

19. (Si oui) Qu'est-ce que le prestataire a fait ?

Le prestataire peut indiquer, directement ou indirectement, à la cliente qu'il se peut qu'elle ait une MST. Soyez attentif au fait qu'il pourrait ne pas mentionner le terme "MST," mais pourrait utiliser l'expression "infection vaginale" ou un autre terme.

Si le prestataire suspecte une MST, la question 19 détermine quelle action, le cas échéant, le prestataire fait pour la cliente. Si aucune action n'a été faite, encerclez 77.

20. Quels sont les autres sujets de santé qui ont été mentionnés à n'importe quel moment de la consultation ?

Le but de cette question est d'évaluer l'intégration des services dans les PPS.

Décision relative à la planification familiale, à l'approvisionnement, et au suivi

21. La cliente a-t-elle décidé d'utiliser une méthode contraceptive ?

Notez cette question seulement pour les nouvelles clientes puisque les anciennes clientes utilisent déjà une méthode. La question 23 traite des anciennes clientes qui changent de méthodes.

22. (Si non) Quelle est la principale raison pour laquelle la cliente n'a pas choisi de méthode?

Si la nouvelle cliente décide de ne pas utiliser de méthode, enregistrez donc son motif ici. Puisque le reste de cette section traite de l'approvisionnement des méthodes ainsi que des arrangements pour le suivi, aucune de ces questions ne s'applique aux clientes qui abandonnent la PF. Si vous avez répondu à la question 22, vous n'avez pas besoin de répondre à une autre question de cette section.

23. Quelle décision la cliente a-t-elle prise par rapport à la planification familiale?

24. (Si arrête ou change) Quelle est la raison principale d'arrêter ou de changer? Ces questions ne concernent que les anciennes clientes. Si la cliente arrête ou change de méthode, essayez de découvrir la raison principale de ce choix et codez en conséquence.

Puisque le reste de cette section traite de l'approvisionnement des méthodes ainsi que des arrangements de suivi, aucune de ces questions ne s'applique aux clientes qui abandonnent la PF. Si vous avez répondu à la question 24 par "arrête," vous n'avez pas besoin de répondre à une autre question de cette section.

25. Pour quelle nouvelle méthode la cliente décide/opte?

Cette question porte sur la méthode que la cliente a finalement choisie, à ne pas confondre avec la méthode qu'elle a préférée au départ, la méthode qu'elle pourrait être en train d'utiliser précédemment, ou la méthode qu'on lui aurait fournie aujourd'hui comme méthode temporaire. Utilisez les codes des méthodes de la page 56.

26. La méthode que la cliente a décidé d'utiliser est-elle la même que celle qu'elle a préférée au départ ?

27. (Si non) Pourquoi la cliente a-t-elle décidé de ne pas utiliser la méthode qu'elle avait préférée initialement?

Si une cliente a une préférence pour une méthode, mais ne la reçoit pas, elle peut se décourager d'utiliser la PF. Vérifiez les questions 10 et 11 pour voir si sa décision finale correspond à sa préférence. 
28. La méthode que la cliente a décidé d'utiliser lui a-t-elle été fournie aujourd'hui?

29. (Si non) Pourquoi la méthode ne lui a pas été fournie aujourd'hui ?

Dans certains cas, la cliente peut décider d'utiliser une méthode, mais en réalité peut ne pas recevoir cette méthode aujourd'hui. Pour cette question, "fournie" veut dire reçoit les pilules ou les préservatifs/spermicide, faire une injection, une pose de DIU ou de NORPLANT $^{\circ}$, subir une ligature des trompes, etc. Si la cliente a choisi la MAMA ou la PF naturelle, ce qui ne nécessite pas de provisions ou de procédures, encerclez "oui."

30. (Si non) Une méthode d'attente a-t-elle été fournie à la cliente en attendant qu'elle reçoive sa méthode choisie?

31. (Si oui) Quelle est la méthode qui a été donnée comme méthode d'attente? Ces questions ne doivent être répondues que lorsque la réponse à la question 28 est "non." Utilisez les codes des méthodes de la page 56.

32. (Si la cliente a choisi la pilule, les préservatifs, les spermicides) Quel est le nombre d'unités de la méthode qui ont été fournies à la cliente ?

Remarquez la description de l'unité utilisée pour chacune de ces méthodes : pilules = cycle mensuel; préservatifs $=$ nombre de pièces individuelles de préservatifs; et spermicides $=$ nombre de flacons de comprimés moussants, etc.

33. A-t-on indiqué à la cliente la date de retour pour un réapprovisionnement ou un suivi?

34. (Si oui) Le prestataire a-t-il donné à la cliente un rappel écrit de la date de retour?

35. La cliente a-t-elle été informée du lieu du réapprovisionnement ou du suivi ?

\section{6. (Si oui) Où ?}

Toutes ces questions de suivi et

d'approvisionnement ont trait à la capacité de la cliente de continuer avec sa méthode une fois qu'elle a commencé à l'utiliser. Des questions similaires sont posées à la cliente dans l'interview de sortie, nous pouvons donc évaluer si les clientes retiennent cette information.

\section{Procédures médicales réalisées}

Faites particulièrement attention aux procédures qui ont été faites sur les clientes, et répondez seulement aux questions qui s'y rapportent. Si la cliente ne reçoit pas de procédure, n'encerclez pas de réponse. Si la cliente reçoit une action mais aucune des actions énumérées n'a été suivie, encerclez le code 77 . Veuillez ne pas encercler 77 pour signifier que la "procédure n'a pas eu lieu."

\section{Si un examen pelvien a été fait, le} prestataire a-t-il :

Cette question n'est utilisée qu'avec les clientes qui ont reçu un examen pelvien. Si le prestataire a utilisé un spéculum, rassurezvous de répondre aussi à la question 38 .

38. (Si un examen à été fait à l'aide d'un spéculum), le prestataire a-t-il : Cette question n'est utilisée qu'avec les clientes chez lesquelles on a utilisé un spéculum pendant l'examen pelvien. Un "spéculum propre" est défini comme étant soit stérile ou "très propre" (désinfecté mais pas nécessairement stérilisé). Souvent il est difficile de dire si le spéculum est propre ou non. Faites le meilleur de votre jugement. Si des gants ont été utilisés, répondez à la question 42 .

39. (Si un DIU a été posé) le prestataire a-t-il : Cette question ne concerne que les clientes qui ont reçu le DIU. Rappelez-vous de votre formation en ce qui concerne les procédures aseptiques et la définition du soutien moral. 
Si des gants ont été utilisés, répondez à la question 42.

40. (Si une injection a été faite), le prestataire a-t-il :

Cette question ne concerne que les clientes qui ont reçu un contraceptif injectable.

41. (Si un NORPLANT ${ }^{\circledR}$ a été posé), le prestataire a-t-il :

Cette question ne concerne que les clientes qui ont reçu le NORPLANT ${ }^{\star}$. Si des gants ont été utilisés, répondez à la question 42 .

\section{2. (Si des gants ont été utilisés), les gants}

\section{étaient-ils :}

Cette question n'est utilisée que dans les situations où le prestataire a utilisé des gants pendant une partie des procédures ou pendant toutes les procédures. "Propre" veut dire très propre ou une nouvelle paire de gants à usage unique. "Stérile" veut dire non déballé mais sorti de l'autoclave. Faites le meilleur de votre jugement, mais si vous avez des doutes, encerclez le code 98.

\section{Fin de l'observation}

\section{Durée de l'interview}

Notez l'heure de la fin de l'observation et vérifiez l'heure du début de l'observation (à la page 50) pour trouver la durée de l'interaction. 
Interview de sortie de la cliente de planification familiale

Cet instrument recueille les informations auprès des clientes PF sur les services qu'elles viennent de recevoir. Cette information est utilisée dans l'évaluation de la qualité des soins que les clientes reçoivent dans les PPS, ce qui peut être comparée à la qualité des soins présentés aux clientes. Cela nous permet de savoir si l'information est communiquée de façon efficace, ce que les clientes pensent des services, et le degré de compréhension des clientes par rapport au processus de sélection d'une méthode de planification familiale.
Les codes présentés entre accolades \{\} dans cet instrument sont des noms de variables proposées pour chaque question ou marque à cocher. On se réfère à ces noms dans le plan d'analyse (commençant à la page 166). Cependant, il faut garder à l'esprit qu'ils n'apparaîtront pas dans le questionnaire utilisé par les chercheurs sur le terrain. Voir les pages qui suivent pour l'instrument. 


\section{Interview de sortie des clientes de planificacion familiale}

INSTRUCTIONS A L'ATTENTION DE L'ENQUETEUR : Copiez l'information de la première page du guide d'observation à cette page de l'interview de sortie. Lire la salutation de la page suivante à la cliente et ne continuer que lorsqu'elle donne son consentement. Pour chacun des éléments du reste de l'interview, encerclez la réponse ou décrivez-la comme appropriée.

Structure sanitaire visitée (nom) :

\{NOMFS\}

Code de la structure sanitaire :

\{CODEFS\}

District (nom) :

\{NOMDIST\}

Code du district :

\{CODEDIST\}

Village/ville (nom) :

\{NOMVILLE\}

Code Village/ville :

\{CODEVILLE\}

Numéro d'étude de la cliente

\{IDCL $\}$

Lieu de la cliente/salle

$\{$ LCL $\}$

Numéro d'étude de l'agent de santé \{IDAS\}

Date de la visite : \{DATE\}

Jour

Mois

Année

Nom de l'observateur :

Signature du chef d'équipe : 
Type de la structure sanitaire: \{TYPE\}

1 = Hôpital de référence

2 = Hôpital de district

3 = Hôpital primaire

4 = Centre de santé rural

5 = Maternité

$6=$ Poste de santé

$7=$ Pharmacie

$8=\mathrm{DBC}$

$9=$ Autre
Type de secteur: $\{$ SECT $\}$

$$
\begin{aligned}
& 1=\text { Gouvernement } \\
& 2=\text { APF } \\
& 3=\text { Mission } \\
& 4=\text { Privé } \\
& 5=\text { Autre }
\end{aligned}
$$

Localité: \{LOC $\}$

1 = Rurale

2 = Urbaine

But principal de la visite comme indiqué prioritairement par la cliente : $\{B U T\}$

Nouvelles clientes : $\quad 1=$ Nouvelle utilisatrice de PF

2 = utilisatrice de PF "Recommençant"

Anciennes clientes: $\quad 3=$ Visite de réapprovisionnement ou visite de suivi (sans problèmes)

4 = Visite de réapprovisionnement ou visite de suivi (problème avec la méthode, veut changer de méthode, ou veut abandonner l'utilisation de la PF) 


\section{POUR TOUTES LES CLIENTES}

\section{Lire les salutations :}

Bonjour Madame. Nous aimerions améliorer les services offerts par la clinique et nous pensons que votre expérience d'aujourd'hui nous serait utile. Je voudrais vous poser quelques questions sur la visite que vous venez juste de faire avec (agent du PPS) et vous serais très reconnaissant de me consacrer quelques minutes pour y répondre. Je ne vais pas relever votre nom, et toutes les informations que vous me donnerez resterons strictement confidentielles. Vous n'êtes pas non plus obligée de répondre à une question si vous n'en avez pas envie, et vous pouvez vous retirer de l'interview à n'importe quel moment. M'autorisez-vous à continuer?

(Si la cliente accepte de continuer, demandez-lui si elle a des questions. Répondez correctement aux questions et ensuite poser la q.1.

\section{Si la cliente refuse de continuer, il faut la remercier et passer à l'interview suivante.)}

1. Dans l'ensemble, êtes-vous satisfaite de votre visite aujourd'hui dans cette structure sanitaire ou n'êtesvous pas satisfaite?

$$
\begin{aligned}
& 1 \text { = Satisfaite (passez à la q.3) } \\
& 2 \text { = Non satisfaite } \\
& 3 \text { = Autre: }
\end{aligned}
$$

2. Pourquoi n'êtes-vous pas satisfaite de votre visite d'aujourd'hui ? \{F2\}

3. En plus de la planification familiale, avez-vous reçu d'autres services de santé de la part du prestataire ?

1 = Oui

2 = Non (passez à la q.5) 


\begin{tabular}{|c|c|}
\hline \multicolumn{2}{|c|}{$\begin{array}{l}\text { 4. A présent j'aimerais vous poser des questions sur tous les services que vous avez reçus aujourd'hui } \\
\text { Avez-vous reçu l'un des services suivants? (Lire de } 1 \text { - 16) }\end{array}$} \\
\hline & Cochez si le service est reçu \\
\hline 1. Soins prénatals & $\{\mathrm{F} 4 \times 1\}$ \\
\hline 2. Soins de maternité/ services d'accouchement & $\{F 4 \times 2\}$ \\
\hline 3. Soins postnatals & $\{\mathrm{F} 4 \times 3\}$ \\
\hline 4. Counseling en VIH/SIDA & $\{\mathrm{F} 4 \times 4\}$ \\
\hline 5. Test de VIH/SIDA & $\{F 4 \times 5\}$ \\
\hline 6. Counseling sur d'autres MST & $\{\mathrm{F} 4 \mathrm{X} 6\}$ \\
\hline 7. Diagnostic d'autres MST & $\{\mathrm{F} 4 \mathrm{X} 7\}$ \\
\hline 8. Traitement d'autres MST & $\{F 4 \times 8\}$ \\
\hline 9. Vaccination de l'enfant & $\{\mathrm{F} 4 \times 9\}$ \\
\hline 10. Surveillance de la croissance de l'enfant & $\{F 4 \times 10\}$ \\
\hline 11. Thérapie de réhydratation orale & $\{\mathrm{F} 4 \mathrm{X} 11\}$ \\
\hline 12. Traitement de l'avortement incomplet & $\{\mathrm{F} 4 \times 12\}$ \\
\hline 13. Counseling en nutrition & $\{\mathrm{F} 4 \mathrm{X} 13\}$ \\
\hline 14. Services curatifs - cliente & $\{F 4 \times 14\}$ \\
\hline 15. Services curatif-enfant & $\{F 4 X 15\}$ \\
\hline 16. Autre: & $\{\mathrm{F} 4 \times 16\}$ \\
\hline
\end{tabular}

5. Avez-vous le sentiment qu'aujourd'hui vous avez reçu l'information et les services que vous vouliez? $\{\mathrm{F} 5\}$

$$
\begin{aligned}
& 1=\text { Oui } \\
& 2=\text { Non } \\
& 3=\text { Un peu } \\
& 98=\text { Ne sait pas }
\end{aligned}
$$

6. Pensez-vous que votre consultation de PF avec l'agent de santé a été trop courte, trop longue, ou juste le temps qu'il faut? $\{$ F6 $\}$

$$
\begin{aligned}
& 1 \text { = Trop courte } \\
& 2=\text { Trop longue } \\
& 3=\text { Juste le temps qu'il faut } \\
& 98=\text { Ne sait pas }
\end{aligned}
$$


7. Pendant la visite, aviez-vous des inquiétudes par rapport à la PF ou d'autres questions de santé que vous vouliez discuter avec le prestataire ? $\quad\{\mathrm{F} 7\}$

$$
\begin{aligned}
& 1 \text { = Oui } \\
& 2 \text { = Non (passez à la q.9) }
\end{aligned}
$$

8. (Si oui) Le prestataire a-t-il répondu à vos préoccupations de manière satisfaisante ? $\{\mathrm{F} 8\}$

$$
\begin{aligned}
& 1=\text { Oui } \\
& 2=\text { Non }
\end{aligned}
$$

9. Pendant cette visite, aviez-vous des questions que vous vouliez poser? $\{\mathrm{F} 9\}$

$$
\begin{aligned}
& 1=\text { Oui } \\
& 2=\text { Non (passez à la q.12) }
\end{aligned}
$$

10. (Si oui) Le prestataire vous a-t-il donné le temps de les poser ? $\{\mathrm{F} 10\}$

1 = Oui

2 = Non (passez à la q.12)

11. (Si oui) Etes-vous satisfaite des réponses que le prestataire a données à vos questions ? $\{$ F11\}

$1=$ Oui

$2=$ Non

12. Pendant cette visite, le prestataire a-t-il fait des examens ou des procédures de santé ? $\{$ F12\}

$1=$ Oui

2 = Non (passez à la q.15)

13. Le prestataire a-t-il expliqué les examens ou procédures avant de les faire ? \{F13\}

$1=$ Oui

$2=$ Non

14. Le prestataire a-t-il expliqué les résultats des examens ou procédures de santé ? \{F14\}

$$
\begin{aligned}
& 1=\text { Oui } \\
& 2=\text { Non }
\end{aligned}
$$

15. A votre avis, y avait-il assez d'intimité pendant votre consultation avec le prestataire de services ? $\{\mathrm{F} 15\}$

$$
\begin{aligned}
& 1=\text { Oui } \\
& 2=\text { Non }
\end{aligned}
$$

16. Au cours de la consultation, avez-vous eu le sentiment que le prestataire était facile à comprendre lorsqu'il vous expliquait certaines choses, ou bien était-il difficile à comprendre ? \{F16\}

$$
\begin{aligned}
& 1 \text { = Facile à comprendre } \\
& 2=\text { Difficile à comprendre } \\
& 98=\text { Ne sait pas }
\end{aligned}
$$


17. Si vous aviez une amélioration à suggérer par rapport aux services fournis, laquelle auriez-vous suggéré ? \{F17\}

18. Au cours de cette visite, vous a-t-on donné ou avez-vous pris un dépliant ou un matériel éducatif pour l'amener à la maison? $\{$ F18\}

$$
\begin{aligned}
& 1 \text { = Oui } \\
& 2=\text { Non (passez à la q.20) } \\
& 98=\text { Ne sait pas (passez à la q.20) }
\end{aligned}
$$

\begin{tabular}{|c|c|}
\hline & Cochez si mentionné \\
\hline 1. Planification familiale & $\{F 19 \times 1\}$ \\
\hline 2. Soins prénatals/Postnatals & $\{F 19 \times 2\}$ \\
\hline 3. Services d'accouchement & $\{F 19 \times 3\}$ \\
\hline 4. $\mathrm{VIH} / \mathrm{SIDA}$ & $\{F 19 \times 4\}$ \\
\hline 5. Autres MST & $\{F 19 X 5\}$ \\
\hline 6. Bien-être de l'enfant & $\{\mathrm{F} 19 \times 6\}$ \\
\hline 7. Nutrition & $\{F 19 X 7\}$ \\
\hline 8. Autre: & $\{F 19 X 8\}$ \\
\hline 98. Ne sait pas & $\{F 19 \times 98\}$ \\
\hline
\end{tabular}

19. (Si oui) Quel est le thème de ce matériel ? (Ne pas lire la liste, mais suscitez davantage de réponses en demandant, "D'autres thèmes?")

20. Avez-vous pris part à une causerie de groupe dans cette structure sanitaire aujourd'hui ? $\{$ F20\} $1=$ Oui

2 = Non (passez à la q.22)

$98=$ Ne sait pas (passez à la q.22) 
21. (Si oui) Quels sont les thèmes qui ont été abordés pendant cette causerie de groupe ?

(Ne pas lire la liste, mais suscitez davantage de réponses en demandant, "D’autres thèmes?")

\begin{tabular}{|c|c|}
\hline & Cochez si mentionné \\
\hline 1. Planification familiale & $\{F 21 \times 1\}$ \\
\hline 2. Soins prénatals & $\{F 21 \times 2\}$ \\
\hline 3. Soins de maternité/services d'accouchement & $\{F 21 \times 3\}$ \\
\hline 4. Soins postnatals & $\{F 21 \times 4\}$ \\
\hline 5. VIH/SIDA & $\{F 21 \times 5\}$ \\
\hline 6. Autres MST & $\{F 21 \times 6\}$ \\
\hline 7. Vaccination de l'enfant & $\{\mathrm{F} 21 \times 7\}$ \\
\hline 8. Surveillance de la croissance de l'enfant. & $\{\mathrm{F} 21 \times 8\}$ \\
\hline 9. Infécondité & $\{\mathrm{F} 21 \times 9\}$ \\
\hline 10. Thérapie de réhydratation orale & $\{F 21 \times 10\}$ \\
\hline 11. Traitement de l'avortement incomplet & \{F21X11\} \\
\hline 12. Counseling en nutrition & $\{\mathrm{F} 21 \times 12\}$ \\
\hline 13. Services curatifs_clientes & $\{F 21 \times 13\}$ \\
\hline 14. Services curatifs - enfants & \{F21X14\} \\
\hline 15. Allaitement & $\{F 21 \times 15\}$ \\
\hline 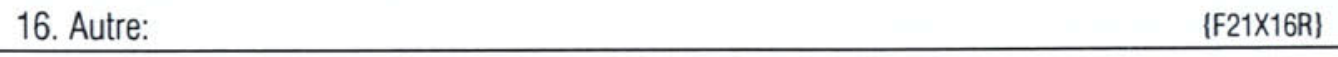 & $\{F 21 \times 16\}$ \\
\hline 98. Ne sait pas & $\{F 21 \times 98\}$ \\
\hline
\end{tabular}

22. Un prestataire de services vous a-t-il dit quand est-ce qu'il faut revenir pour une autre visite ? \{F22\}

1 = Oui

$2=$ Non

$98=$ Ne sait pas

23. Les heures d'ouverture de ce PPS sont-elles convenables pour vous ? $\{$ F23\}

1 = Oui (passez à la q.25)

$2=$ Non

$98=$ Ne sait pas (passez à la q.25) 
24. (Si non) Quel est le l'heure qui vous conviendrait le mieux ? (Encerclez un.) \{F24\}

1 = Tôt le matin

2 = Pendant l'heure du repas

3 = L'après-midi

$4=$ Le soir/la nuit

5 = Les week-ends / jours fériés

6 = Autre :

$98=$ Ne sait pas

25. Avez-vous jamais été renvoyée de cette structure sanitaire pendant les heures officielles de travail?

1 = Oui

$2=$ Non

3 = Pas d'expérience précédente avec la structure sanitaire

$98=$ Ne sait pas

26. Environ combien de temps vous a-t-il fallu attendre entre le moment où vous êtes arrivée à ce PPS et celui auquel vous avez commencé à recevoir les services pour lesquels vous êtes venue? \{F26\} minutes

$98=$ Ne sait pas $\quad$ (S'il n'y a pas de temps d'attente, passez à la q.28)

27. Avez-vous le sentiment que votre temps d'attente était raisonnable ou trop long ? $\{$ F27\}

1 = Raisonnable

2 = Trop long

$98=$ Ne sait pas

28. Combien de temps vous a-t-il fallu pour arriver ici aujourd'hui ? $\{$ F28 $\}$ minutes

$98=$ Ne sait pas

29. Quel a été le principal moyen de transport que vous avez utilisé pour venir ici ? (Encerclez un) \{F29\}

$1=$ A pieds

2 = Charrette

$3=$ Bus $/$ combi

4 = Bicyclette

$5=$ Voiture personnelle

6 = Autre : 
30. A votre connaissance, quels types de services autres que la planification familiale sont généralement fournis dans cette structure sanitaire? (Ne pas lire, mais suscitez davantage de réponses en demandant, "D'autres services?")

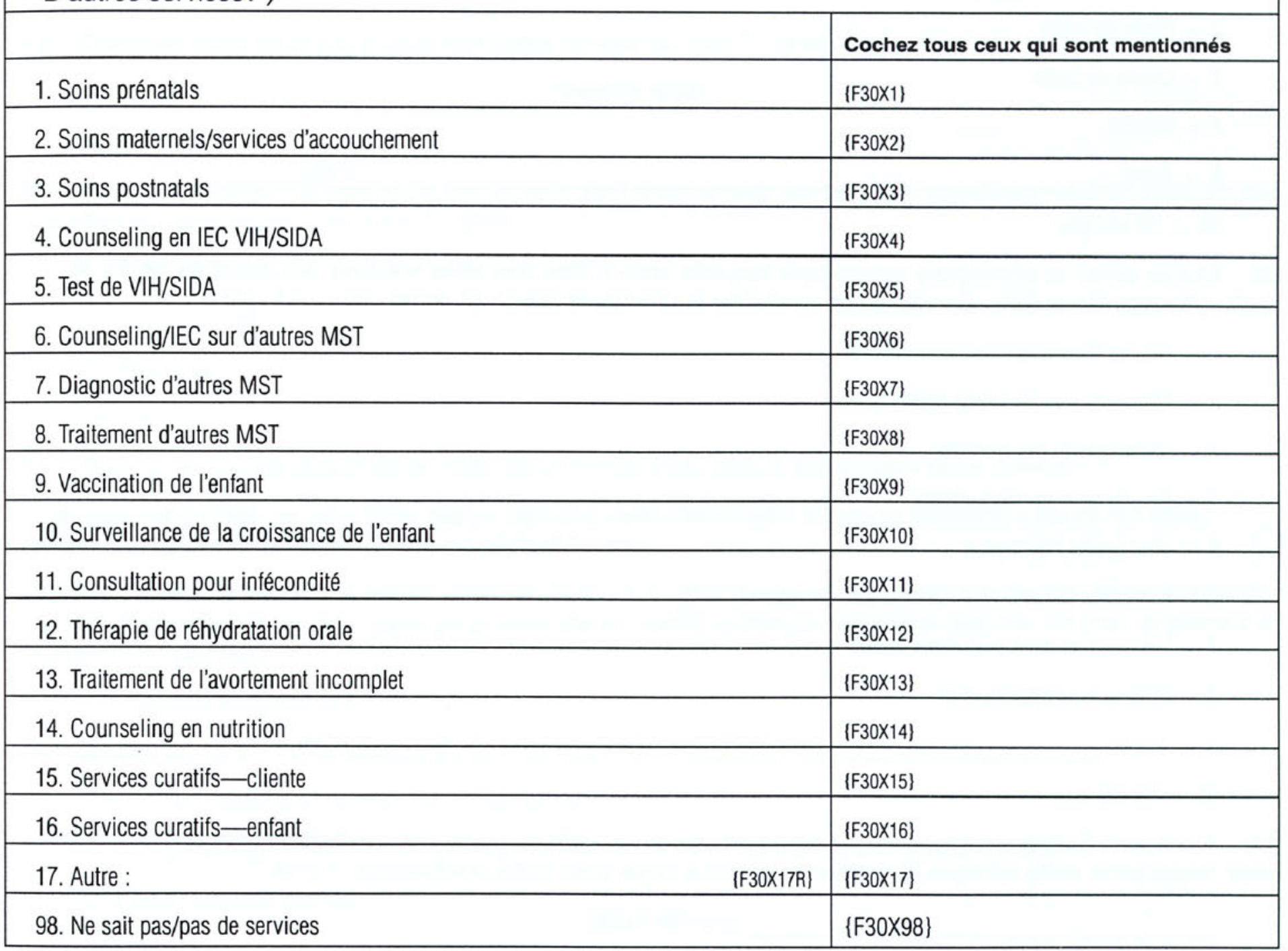

31. En dehors de ce PPS, y a-t-il un autre endroit près de chez vous où vous pouvez obtenir des services de planification familiale?

$$
\begin{aligned}
& 1 \text { = Oui } \\
& 2=\text { Non (passez à la q.34) } \\
& 98=\text { Ne sait pas (passez à la q.34) }
\end{aligned}
$$


32. (Si oui) Quel est le type de cette structure sanitaire? (Encerclez un. S'il y a en plusieurs, choisissez la plus proche de chez elle.) $\{\mathrm{F} 32\}$

$1=\mathrm{CBD}$

2 = Poste de santé

3 = Centre de santé

4 = Hôpital

6 = Autre : [F32R\}

$98=$ Ne sait pas

33. Quelle serait la principale raison pour laquelle vous n'êtes pas allée solliciter des services de PF làbas? (Ne pas lire la liste. Sondez pour connaître la principale raison et encerclez une.) \{F33\}

$1=$ Heures d'ouverture non convenables

2 = Nécessite trop de temps pour y arriver

3 = Piètre qualité des services

4 = Peu de services disponibles

5 = Veut garder l'anonymat

6 = A d'autres raisons pour venir ici (ex. services de SMI)

7 = Les services sont plus chers là-bas

8 = Préfère le prestataire d'ici

6 = Autre : \{F33R\}

$98=$ Ne sait pas

34. A présent, j'aimerais vous poser des questions sur le coût de votre transport et les services que vous avez reçus dans cette clinique. Combien avez-vous payé pour votre consultation ? \{F34\} (monnaie locale)

98 = Ne sait pas

35. Combien avez-vous payé pour les médicaments ? $\{F 35\}$ (monnaie locale)

98 = Ne sait pas

36. Combien avez-vous payé pour les autres produits tels que le coton, les gants, les produits antiseptiques ou les seringues? $\{\mathrm{F} 36\}$ (monnaie locale)

$98=$ Ne sait pas

37. Combien avez-vous payé pour la carte d'inscription/de membre ? \{F37\} (monnaie locale)

$98=$ Ne sait pas 
38. Combien avez-vous payé pour le transport ? $\{\mathrm{F} 38\}$ (monnaie locale)

$98=$ Ne sait pas

39. Combien avez-vous payé pour tout autre service ou frais ? $\{F 39\}$ (monnaie locale)

$98=$ Ne sait pas

40. Dans l'ensemble, pensez-vous que le coût total pour obtenir les services est beaucoup trop cher, un peu trop cher ou acceptable pour vous? $\{\mathrm{F} 40\}$

1 = Beaucoup trop cher

2 = Un peu trop cher

$3=$ Acceptable

$98=$ Ne sait pas

41. (Voir la principale raison de la visite de la cliente à la page 2, lire ensuite cette question.)

Si je comprends bien, la principale raison de votre visite dans cette structure sanitaire aujourd'hui était (insérer la raison de la page 2). Est-ce correct? $\{\mathrm{F} 41\}$

(Si c'est correct, encerclez la même réponse de la page 2, et passez à la question indiquée. Si ce n'est pas correct, demandez la bonne raison de la visite de la cliente, encerclez la bonne réponse en bas, et passez à la question indiquée.)

1 = Nouvelle acceptante de PF

2 = Utilisatrice de PF "Recommençant"

3 = Visite de réapprovisionnement ou de suivi (passez à la q.55)

4 = Problème avec la méthode, voulait changer de méthode, ou voulait abandonner la PF (passez à la q.59)

5 = Autre (passez à la q.101) 


\section{POUR LES NOUVELLES ACCEPTANTES}

42. Juste pour me rassurer, je comprends que vous n'utilisiez pas de méthode contraceptive avant de venir aujourd'hui dans cette structure sanitaire. Est-ce exact que vous n'utilisiez pas de méthode contraceptive? $\{\mathrm{F} 42\}$

$1=$ Non, n'utilisait pas

2 = Oui, utilisait (retournez à la q.41)

43. Avez-vous déjà utilisé une méthode contraceptive dans le passé ?

$1=$ Oui

$2=$ Non (passez à la q.45)

44. (Si oui) Quelle(s) méthode(s) avez-vous déjà utilisée(s) ? (Ne pas lire les méthodes mais suscitez davantage de réponses en demandant, "Avez-vous déjà utilisé une autre méthode ?")

\begin{tabular}{|c|c|}
\hline & Cochez si la méthode est mentionnée \\
\hline 1. Pilule combinée (ou non spécifiée) & $\{\mathrm{F} 44 \times 1\}$ \\
\hline 2. Pilule progestative & $\{F 44 \times 2\}$ \\
\hline 3. DIU & $\{\mathrm{F} 44 \times 3\}$ \\
\hline 4. Injectable & $\{\mathrm{F} 44 \times 4\}$ \\
\hline 5. NORPLANT ${ }^{\circledast}$ & $\{\mathrm{F} 44 \times 5\}$ \\
\hline 6. Préservatif & $\{F 44 X 6\}$ \\
\hline 7. Diaphragme & $\{F 44 X 7\}$ \\
\hline 8. Spermicide & $\{F 44 \times 8\}$ \\
\hline 9. Ligature des trompes (ML/AL) & $\{\mathrm{F} 44 \times 9\}$ \\
\hline 10. Vasectomie & $\{\mathrm{F} 44 \times 10\}$ \\
\hline 11. PFN & $\{F 44 \times 11\}$ \\
\hline 12. MAMA & $\{\mathrm{F} 44 \times 12\}$ \\
\hline 13. Autre : & $\{\mathrm{F} 44 \times 13\}$ \\
\hline 98. Ne sait pas & $\{F 44 \times 98\}$ \\
\hline
\end{tabular}

45. Avez-vous reçu une méthode contraceptive au cours de cette visite ? $\{$ F45 $\}$

1 = Oui (passez à la q.47)

$2=$ Non 
46. (Si non) Quelle est la raison principale pour laquelle vous n'avez pas obtenu de méthode contraceptive aujourd'hui ? (Encerclez une.) $\{\mathrm{F} 46\}$

1 = Venue s'informer seulement (passez à la q.101)

2 = Changé d'avis (passez à la q.101)

3 = Grossesse suspectée (passez à la q.101)

4 = Contre-indications pour la méthode choisie (passez à la q.101)

5 = Continue à allaiter (passez à la q.101)

6 = Autre raison de santé (passez à la q.101)

7 = Méthode non disponible (passez à la q.101)

8 = Autre : (passez à la q.101)

98 = Ne sait pas (passez à la q.101)

47. Quelle(s) méthode(s) avez-vous acceptée(s) aujourd'hui ? (Ne pas lire les méthodes mais suscitez davantage de réponses en demandant, "Avez-vous accepté une autre méthode?")

\begin{tabular}{|c|c|}
\hline & Cochez si la méthode est mentionnée \\
\hline 1. Pilule combinée (ou non spécifiée) & $\{\mathrm{F} 47 \mathrm{X} 1\}$ \\
\hline 2. Pilule progestative & $\{\mathrm{F} 47 \times 2\}$ \\
\hline 3. DIU & $\{\mathrm{F} 47 \times 3\}$ \\
\hline 4. Injectable & $\{\mathrm{F} 47 \times 4\}$ \\
\hline 5. NORPLANT ${ }^{\circledR}$ & $\{F 47 \times 5\}$ \\
\hline 6. Préservatif & $\{F 47 \times 6\}$ \\
\hline 7. Diaphragme & $\{\mathrm{F} 47 \times 7\}$ \\
\hline 8. Spermicide & $\{F 47 \times 8\}$ \\
\hline 9. Ligature des trompes (ML/AL) & $\{\mathrm{F} 47 \times 9\}$ \\
\hline 10. Vasectomie & $\{F 47 \times 10\}$ \\
\hline 11. PFN & $\{\mathrm{F} 4 \mathrm{XX11 \}}$ \\
\hline 12. MAMA & $\{\mathrm{F} 47 \times 12\}$ \\
\hline 13. Autre: & $\{\mathrm{F} 47 \times 13\}$ \\
\hline 98. Ne sait pas & \{F47X98\} \\
\hline
\end{tabular}


48. Le prestataire vous a-t-il parlé d'une autre méthode? $\{$ F48 $\}$

$$
\begin{aligned}
& 1=\text { Oui } \\
& 2=\text { Non } \\
& 98=\text { Ne sait pas }
\end{aligned}
$$

49. Le prestataire vous a-t-il dit que vous pouvez changer de méthode si vous n'êtes pas satisfaite de celle-ci ? $\{$ F49\}

$$
\begin{aligned}
& 1=\text { Oui } \\
& 2=\text { Non } \\
& 98=\text { Ne sait pas }
\end{aligned}
$$

50. Le prestataire vous a-t-il indiqué une autre source d'approvisionnement ou de suivi pour votre méthode? $\{$ F50\}

$$
\begin{aligned}
& 1=\text { Oui } \\
& 2=\text { Non } \\
& 98=\text { Ne sait pas }
\end{aligned}
$$

51. Y a-t-il une autre méthode différente de celle que vous avez acceptée aujourd'hui et que vous préféreriez utiliser? \{F51\}

$$
\begin{aligned}
& 1 \text { = Oui } \\
& 2 \text { = Non (passez à la q.54) } \\
& 98 \text { = Ne sait pas(passez à la q.54) }
\end{aligned}
$$

52. (Si oui) Quelle méthode préfériez-vous ? (Encerclez une.) \{F52\}

1. Pilule combinée (ou non spécifiée)

2. Pilule progestative

3. DIU

4. Injectable

5. NORPLANT ${ }^{\oplus}$

6. Préservatif

7. Diaphragme

8. Spermicide

9. Ligature des trompes (ML/AL)

10. Vasectomie

11. PFN

12. MAMA

13. = Autre :

98. Ne sait pas 
53. Pourquoi n'allez-vous pas utiliser cette méthode préférée? (Ne pas lire la liste, mais suscitez davantage de réponses en demandant "Autres raisons ?")

\begin{tabular}{|c|c|}
\hline & Cochez si la raison est mentionnée \\
\hline 1. Le prestataire a dit qu'elle n'est pas appropriée & $\{F 53 X 1\}$ \\
\hline 2. La méthode n'est pas offerte dans cette structure sanitaire & $\{\mathrm{F} 53 \times 2\}$ \\
\hline 3. Le prestataire formé est absent & $\{\mathrm{F} 53 \times 3\}$ \\
\hline 4. La méthode est temporairement en rupture de stock & $\{\mathrm{F} 53 \times 4\}$ \\
\hline $\begin{array}{ll} & \\
{[F 53 \times 5 R\}}\end{array}$ & $\{F 53 \times 5\}$ \\
\hline 98. Ne sait pas & \{F53X98\} \\
\hline
\end{tabular}

54. (Encerclez la méthode la plus efficace acceptée par la cliente, à partir de la q. 47.) A présent, je voudrais vous demander de m'expliquer comment utiliser (lire la méthode). \{F54\}

1 = Pilule combinée (passez à la q.72)

2 = Pilule progestative (passez à la q.72)

3 = DIU (passez à la q.81)

4 = Injectable (passez à la q.89)

$5=$ NORPLANT $^{\circledast}$ (passez à la q.101)

6 = Préservatif (passez à la q.97)

7 = Diaphragme (passez à la q.101)

8 = Spermicide (passez à la q.97)

9 = Ligature des trompes (passez à la q.101)

10 = Vasectomie (passez à la q.101)

$11=$ PFN (passez à la q.101)

$12=$ MAMA (passez à la q.101)

13 = Autre :

(passez à la q.101) $\quad$ \{F54R $\}$ 


\section{POUR LES ANCIENNES CLIENTES QUI N'ONT PAS DE PROBLÈMES}

55. Juste pour me rassurer, je comprends que vous utilisiez une méthode contraceptive avant de venir dans cette structure sanitaire aujourd'hui. Est-ce correct que vous utilisiez une méthode?

1 = Oui, utilisait une

2 = Non, n'en utilisait pas (retournez à la q.41)

56. Quelle(s) méthode(s) utilisiez-vous ? (Ne pas lire les méthodes mais suscitez davantage de réponses en demandant, "Autres méthodes ?")

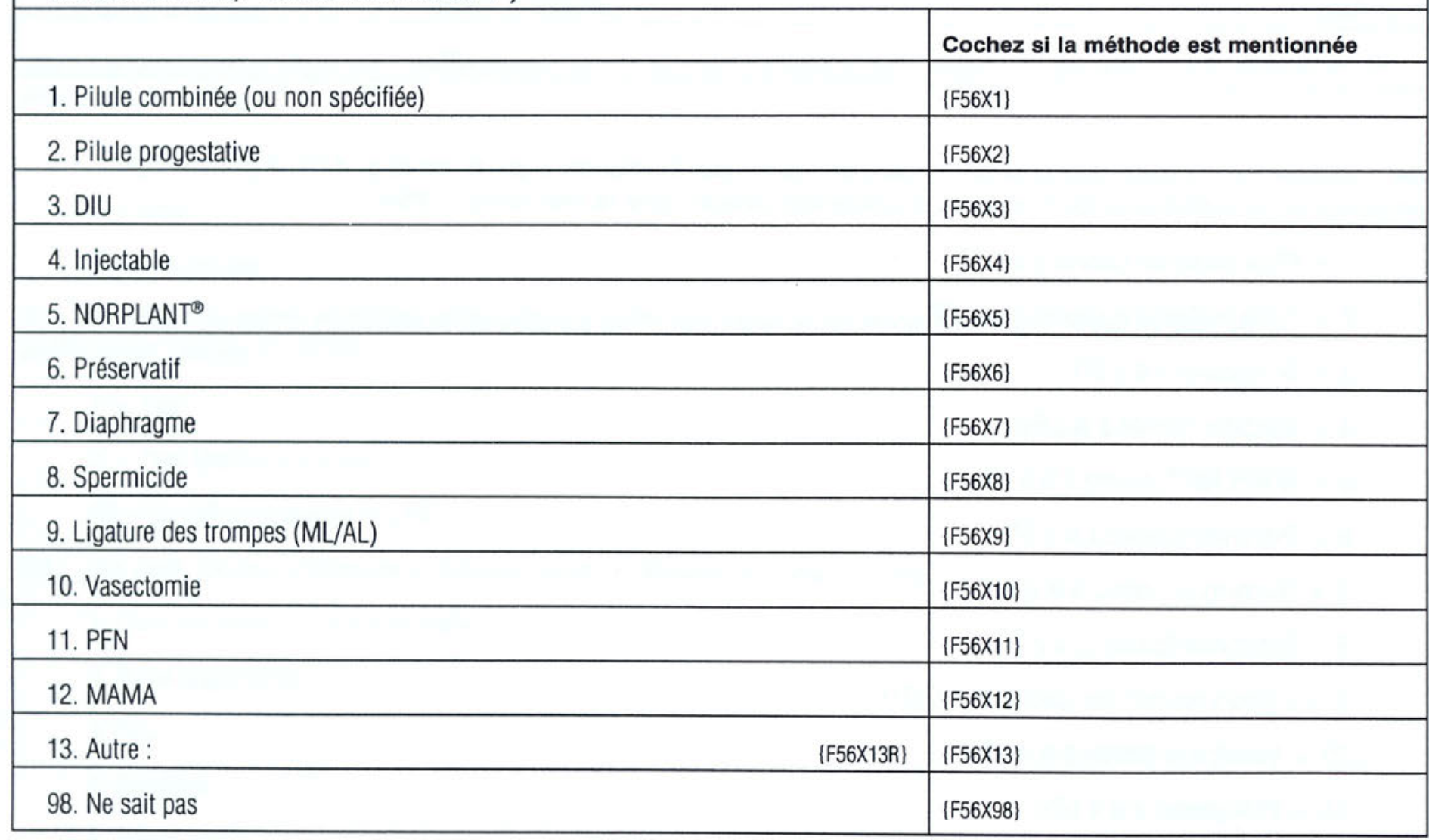

57. Avez-vous l'intention de continuer à utiliser cette méthode ?

$$
\begin{aligned}
& 1 \text { = Oui } \\
& 2=\text { Non (passez à la q.62) } \\
& 98=\text { Ne sait pas (passez à la q.101) }
\end{aligned}
$$


58. (Encerclez la méthode efficace la plus utilisée par la cliente, à partir de la q. 56 ou 60.) Je voudrais vous demander maintenant comment utiliser (lire la méthode).

1 = Pilule combinée (passez à la q.72)

2 = Pilule progestative (passez à la q.72)

3 = DIU (passez à la q.81)

4 = Injectable (passez à la q.89)

$5=$ NORPLANT $^{\circledast}$ (passez à la q.101)

6 = Préservatif (passez à la q.97)

7 = Diaphragme (passez à la q.101)
8 = Spermicide (passez à la q.97)

9 = Ligature des trompes (passez à la q.101)

10 = Vasectomie (passez à la q.101)

11 = PFN (passez à la q.101)

$12=$ MAMA (passez à la q.101)

13 = Autre (passez à la q.101) 


\section{POUR LES ANCIENNES CLIENTES QUI ONT DES PROBLÈMES AVEC LA MÉTHODE, VEULENT CHANGER OU ARRÊTER}

59. Juste pour me rassurer, je comprends que vous utilisiez une méthode contraceptive avant de venir dans cette structure sanitaire aujourd'hui. Est-ce correct que vous utilisiez une méthode?

$$
\begin{aligned}
& 1=\text { Oui, utilisait une } \\
& 2=\text { Non, n'en utilisait pas (retournez à la q.41) }
\end{aligned}
$$

60. Quelle(s) méthode(s) utilisiez-vous ? (Ne pas lire les méthodes mais suscitez davantage de réponses en demandant, "Autres méthodes ?")

\begin{tabular}{|l|l|}
\hline & Cochez si la méthode est mentionnée \\
\hline 1. Pilule combinée (ou non spécifiée) & $\{\mathrm{F} 60 \times 1\}$ \\
\hline 2. Pilule progestative & $\{\mathrm{F} 60 \times 2\}$ \\
\hline 3. DIU & $\{\mathrm{F} 60 \times 3\}$ \\
\hline 4. Injectable & $\{\mathrm{F} 60 \times 4\}$ \\
\hline 5. NORPLANT ${ }^{\circ}$ & $\{\mathrm{F} 60 \times 5\}$ \\
\hline 6. Préservatif & $\{\mathrm{F} 60 \times 6\}$ \\
\hline 7. Diaphragme & $\{\mathrm{F} 60 \times 7\}$ \\
\hline 8. Spermicide & $\{\mathrm{F} 60 \times 8\}$ \\
\hline 9. Ligature des trompes (ML/AL) & $\{\mathrm{F} 60 \times 9\}$ \\
\hline 10. Vasectomie & $\{\mathrm{F} 60 \times 10\}$ \\
\hline 11. PFN & $\{\mathrm{F} 60 \times 11\}$ \\
\hline 12. MAMA & $\{\mathrm{F} 60 \times 12\}$ \\
\hline 13. Autre: & $\{\mathrm{F} 60 \times 31\}$ \\
\hline 98. Ne sait pas & $\{\mathrm{F} 60 \times 98\}$ \\
\hline
\end{tabular}

61. Est-ce vrai que vous aviez un problème, que vous vouliez changer de méthodes ou arrêter d'utiliser la planification familiale? \{F61\}

$$
\begin{aligned}
& 1=\text { Oui } \\
& 2=\text { Non (retournez à la q.57) }
\end{aligned}
$$


62. Quel est le problème majeur que vous aviez, ou la principale raison pour laquelle vous vouliez changer ou arrêter l'utilisation de la PF ? (Encerclez une.) \{F62\}

1 = Effets secondaires médicaux

2 = Mari/partenaire n'aimait pas la méthode (passez à la q.65)

3 = Pression exercée par les autres (passez à la q.65)

4 = Peur de l'infécondité (passez à la q.65)

5 = Voulait une grossesse (passez à la q.65)

6 = N'aimait pas la méthode (passez à la q.65)

7 = Méthode non disponible / difficile à obtenir (passez à la q.65)

8 = Autre : (passez à la q.65) $\quad$ \{F62R\}

98 = Ne sait pas (passez à la q.65)

63. (Si avait des effets secondaires médicaux) Quand vous avez décidé pour la première fois d'utiliser cette méthode, vous avait-on dit que ce problème pourrait arriver ?

1 = Oui

$2=$ Non

$98=$ Ne sait pas

64. (Si oui) Vous avait-on dit ce qu'il fallait faire si ce problème survenait ? $\{$ F64 $\}$

$1=$ Oui

$2=$ Non

$98=$ Ne sait pas

65. Après cette consultation aujourd'hui, avez-vous le sentiment que vous avez reçu l'assistance appropriée pour vos besoins particuliers? \{F65\}
1 = Oui
$2=$ Non
$3=$ un peu
98 = Ne sait pas

66. Qu'allez-vous faire maintenant de la planification familiale ? (Encerclez un choix.) \{F66\}

1 = Changer pour une nouvelle méthode

2 = Continuer avec la même méthode (passez à la q.71)

3 = Arrêter l'utilisation de toute méthode (passez à la q.101)

98 = Ne sait pas (passez à la q.71) 
67. (Si change de méthode) Quelle(s) méthode(s) utiliserez-vous maintenant ? (Ne pas lire les méthodes mais suscitez davantage de réponses en demandant, "Autres méthodes?")

\begin{tabular}{|l|l|}
\hline & Cochez si la méthode est mentionnée \\
\hline 1. Pilule combinée (ou non spécifiée) & $\{67 \times 1\}$ \\
\hline 2. Pilule progestative & $\{67 \times 2\}$ \\
\hline 3. DIU & $\{67 \times 3\}$ \\
\hline 4. Injectable & $\{67 \times 4\}$ \\
\hline 5. NORPLANT ${ }^{\oplus}$ & $\{67 \times 5\}$ \\
\hline 6. Préservatif & $\{67 \times 6\}$ \\
\hline 7. Diaphragme & $\{67 \times 7\}$ \\
\hline 8. Spermicide & $\{67 \times 8\}$ \\
\hline 9. Ligature des trompes (ML/AL) & $\{67 \times 9\}$ \\
\hline 10. Vasectomie & $\{67 \times 10\}$ \\
\hline 11. PF naturelle & $\{67 \times 11\}$ \\
\hline 12. MAMA & $\{67 \times 12\}$ \\
\hline 13. Autre: & $\{67 \times 13\}$ \\
\hline 98. Ne sait pas & $\{67 \times 98\}$ \\
\hline
\end{tabular}

68. Le prestataire vous a-t-il parlé d'autres méthodes ? $\quad\{$ F68 $\}$

$$
\begin{aligned}
& 1=\text { Oui } \\
& 2=\text { Non } \\
& 98=\text { Ne sait pas }
\end{aligned}
$$

69. Le prestataire vous a-t-il dit que vous pouvez changer de méthodes si vous n'êtes pas satisfaite de celle-ci ? $\{$ F69\}

$$
\begin{aligned}
& 1=\text { Oui } \\
& 2=\text { Non } \\
& 98=\text { Ne sait pas }
\end{aligned}
$$

70. Le prestataire vous a-t-il indiqué une autre source d'approvisionnement ou de suivi pour votre méthode? $\{F 70\}$

$$
\begin{aligned}
& 1=\text { Oui } \\
& 2=\text { Non } \\
& 98=\text { Ne sait pas }
\end{aligned}
$$


71. (Encerclez la méthode efficace la plus utilisée maintenant par la cliente, à partir de la q.60 ou la q.67.) Je voudrais vous demander maintenant comment utiliser (lire la méthode). $\quad$ FF71\}

1 = Pilule combinée (passez à la q.72)

2 = Pilule progestative (passez à la q.72)

3 = DIU (passez à la q.81)

4 = Injectable (passez à la q.89)

$5=$ NORPLANT $^{\oplus}$ (passez à la q.101)

6 = Préservatif (passez à la q.97)

7 = Diaphragme (passez à la q.101)

8 = Spermicide (passez à la q.97)

9 = Ligature des trompes (passez à la q.101)

$10=$ Vasectomie (passez à la q.101)

11 = PFN (passez à la q.101)

$12=$ MAMA (passez à la q.101)

13 = Autre : (passez à la q.101) \{F62\} 


\section{POUR LES UTILISATRICES DE LA PILULE}

72. Quand une femme se met sous pilule pour la première fois, à quel moment du cycle menstruel pensezvous qu'elle devrait commencer la prise? $\{$ F72 $\}$

1 = Entre le 1er et le 5eme jour de la période menstruelle

2 = Toute autre réponse

$98=$ Ne sait pas

73. A quelle fréquence pensez-vous qu'une femme devrait prendre la pilule ? $\{$ F73\}

1 = Une pilule tous les jours

2 = Toute autre réponse

$98=$ Ne sait pas

74. Selon vous, si une femme oublie de prendre la pilule un jour, que devrait-elle faire? $\{$ F74 $\}$

1 = Prendre immédiatement la pilule oubliée

2 = Toute autre réponse

$98=$ Ne sait pas

75. La pilule protège-t-elle la femme contre les MSTNIH ? $\{$ F75

1 = Oui

2 = Partiellement

$3=$ Non

$98=$ Ne sait pas

76. A votre connaissance, quels sont les problèmes, s'il y en a, qu'une femme peut avoir en prenant la pilule? (Ne pas lire la liste, mais suscitez davantage de réponses en demandant, "Autres problèmes ?")

\begin{tabular}{|c|c|}
\hline & Cochez tous ceux qui sont mentionnés \\
\hline 1. Nausée & $\{\mathrm{F} 76 \times 1\}$ \\
\hline 2. Légers maux de tête & $\{F 76 \times 2\}$ \\
\hline 3. Saignottement / saignement & $\{F 76 \times 3\}$ \\
\hline 4. Gain/perte de poids & $\{F 76 \times 4\}$ \\
\hline 5. Cancer & $\{\mathrm{F} 76 \times 5\}$ \\
\hline 6. Infécondité & $\{F 76 \times 6\}$ \\
\hline 7. Autre: & $\{F 76 X 7\}$ \\
\hline 77. Aucun problème mentionné & $\{F 76 \times 77\}$ \\
\hline 98. Ne sait pas & $\{F 76 \times 98\}$ \\
\hline
\end{tabular}


77. En dehors de la visite régulière de réapprovisionnement, à votre avis quels sont les problèmes, s'il y en a, pour lesquels une femme sous pilule devrait revenir au PPS ? (Ne pas lire la liste, mais suscitez davantage de réponses en demandant, "Autres problèmes ?")

\begin{tabular}{|c|c|}
\hline & Cochez si mentionné \\
\hline 1. Douleur thoracique aiguë, difficultés respiratoires & $\{F 77 \times 1\}$ \\
\hline 2. Violents maux de tête & $\{F 77 \times 2\}$ \\
\hline 3. Baisse de la vision ou vision floue & $\{F 77 \times 3\}$ \\
\hline 4. Douleurs abdominale aiguës & $\{F 77 \times 4\}$ \\
\hline 5. Douleurs aiguës aux jambes & $\{F 77 \times 5\}$ \\
\hline 6. Règles tardives & $\{F 77 \times 6\}$ \\
\hline$\{$ F77X7\} & $\{F 77 \times 7\}$ \\
\hline 77. Aucun problème mentionné & $\{F 77 \times 77\}$ \\
\hline 98. Ne sait pas & $\{F 77 \times 98\}$ \\
\hline
\end{tabular}

78. Le prestataire vous a-t-il donné une provision de pilules aujourd'hui ? \{F78\}

1 = Oui (passez à la q.80)

$2=$ Non

79. (Si non) Où irez-vous vous approvisionner en pilules ? (Encerclez une.)

1 = Dans une autre section de cette structure sanitaire aujourd'hui

2 = Reviendra dans cette formation une autre fois

3 = Une autre structure sanitaire

4 = Pharmacie / boutique / pharmacien / médecin privé

$5=\mathrm{DBC}$

6. = Autre :

$98=$ Ne sait pas

80. Passez à la q.101 


\section{POUR LES UTILISATRICES DU DIU}

81. Votre visite d'aujourd'hui dans cette structure sanitaire a-t-elle porté sur la pose, le retrait, ou un suivi de routine du DIU? $\{$ F81\}

1 = DIU posé aujourd'hui

2 = Retiré

3 = Suivi de routine (précédemment posé)

82. A votre connaissance, comment une femme peut vérifier pour être sûre que le DIU est en place ? (Encerclez toutes les réponses applicables.) $\quad$ \{F82\}
1 = Vérifier les fils régulièrement
\{F82X1\}
2 = Aller dans le PPS
$\{\mathrm{F} 82 \mathrm{X} 2\}$
3 = Toute autre réponse
\{F82X3\}
$98=$ Ne sait pas
\{F82X98\}

83. A votre connaissance, quels sont les problèmes, s'il y en a, qu'une femme peut avoir avec le DIU ? (Ne pas lire la liste, mais suscitez davantage de réponses en demandant, "Autres problèmes?")

\begin{tabular}{|c|c|}
\hline & Cochez tous ceux qui sont mentionnés \\
\hline 1. Crampes abdominales & $\{F 83 X 1\}$ \\
\hline 2. Saignement abondant & $\{\mathrm{F} 83 \times 2\}$ \\
\hline 3. Saignottement entre les règles & $\{\mathrm{F} 83 \times 3\}$ \\
\hline 4. Douleur dorsale / maux de reins & $\{F 83 \times 4\}$ \\
\hline 5. Infection (IP) / douleurs au bas ventre & $\{F 83 \times 5\}$ \\
\hline 6. Infécondité & $\{F 83 \times 6\}$ \\
\hline 7. Pertes blanches abondantes & $\{\mathrm{F} 83 \times 7\}$ \\
\hline 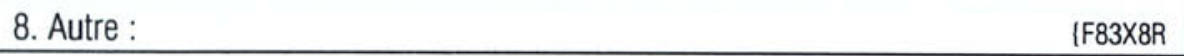 & $\{F 83 \times 8\}$ \\
\hline 77. Aucun problème mentionné & \{F83X77\} \\
\hline 98. Ne sait pas & \{F83X98\} \\
\hline
\end{tabular}

84. A votre avis, après la pose du DIU, quand une femme devrait-elle revenir pour la première visite de contrôle? (Encerclez une réponse.) \{F84\}
1 = Après un mois
2 = Après trois mois
$3=$ Après un an
4 = A n'importe quel autre moment
5 = N'a pas besoin de revenir
$98=$ Ne sait pas 
85. En dehors des visites régulières de contrôle, quels sont les problèmes, s'il y en a, pour lesquels vous pensez qu'une femme portant le DIU devrait revenir dans la structure sanitaire ? (Ne pas lire la liste, mais suscitez davantage de réponses en demandant, "Autres problèmes?")

\begin{tabular}{|c|c|}
\hline & Cochez tous ceux qui sont mentionnés \\
\hline 1. Pertes blanches abondantes & $\{\mathrm{F} 85 \mathrm{X} 1\}$ \\
\hline 2. Saignottement ou saignement anormal & $\{\mathrm{F} 85 \times 2\}$ \\
\hline 3. Expulsion ou ne peut pas sentir les fils & $\{F 85 \times 3\}$ \\
\hline 4. Douleur au bas ventre & $\{\mathrm{F} 85 \times 4\}$ \\
\hline 5. Crampes graves & $\{\mathrm{F} 85 \times 5\}$ \\
\hline 6. Douleur pendant les rapports sexuels & $\{\mathrm{F} 85 \times 6\}$ \\
\hline 7. Douleurs dorsales & $\{F 85 \times 7\}$ \\
\hline 8. Règles tardives & $\{F 85 \times 8\}$ \\
\hline 9. Ne se sent pas bien & $\{\mathrm{F} 85 \times 9\}$ \\
\hline 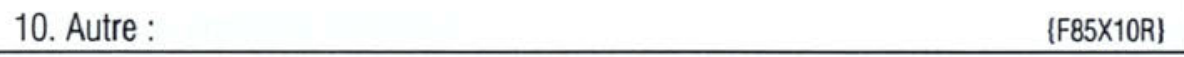 & $\{F 85 \times 10\}$ \\
\hline 77. Aucun problème mentionné & $\{F 85 \times 77\}$ \\
\hline 98. Ne sait pas & $\{F 85 \times 98\}$ \\
\hline
\end{tabular}

86. Pendant combien d'années pensez-vous qu'une femme peut continuer à utiliser son DIU une fois qu'il est posé ? \{F86\}

$$
\text { ans } \quad 98=\text { Ne sait pas }
$$

87. Le DIU protège-t-il la femme contre les MSTNIH ? \{F87\}

$$
\begin{aligned}
& 1 \text { = Oui } \\
& 2=\text { Partiellement } \\
& 3=\text { Non } \\
& 98=\text { Ne sait pas }
\end{aligned}
$$

88. Passez à la q.101 


\section{POUR LES UTILISATRICES D'INJECTABLES}

89. Avez-vous reçu une injection aujourd'hui ? $\{\mathrm{F} 89\}$

1 = Oui (passez à la q.91)

$2=$ Non

90. (Si non) Où irez-vous pour prendre votre injection? (Encerclez une réponse.)

1 = Dans une autre section de cette structure sanitaire aujourd'hui

2 = Reviendra dans cette formation une autre fois

3 = Une autre structure sanitaire

4 = Pharmacie / boutique / pharmacien / médecin privé

$5=\mathrm{DBC}$

6. = Autre :

$98=$ Ne sait pas

91. Quel type d'injection avez-vous reçue ou recevrez-vous ? (Encerclez une.) \{F91\}

1 = Depo Provera

$2=$ Noristerat

$98=$ Ne sait pas

92. A votre avis, à quelle fréquence devriez-vous prendre cette injection ? (Encerclez une.) \{F92\}

$1=$ Chaque mois

2 = Tous les deux mois

$3=$ Tous les trois mois

4 = Tout autre intervalle

98 = Ne sait pas

93. A votre connaissance, quels sont les problèmes, s'il y en a, qu'une femme peut avoir après avoir reçu une injection? (Ne pas lire la liste, mais suscitez davantage de réponses en demandant, "Autres problèmes?")

\begin{tabular}{|c|c|}
\hline & Cocher tous ceux qui sont mentionnés \\
\hline 1. Légers maux de tête & $\{F 93 \times 1\}$ \\
\hline 2. Nausée & $\{\mathrm{F} 93 \times 2\}$ \\
\hline 3. Saignement/saignottement irrégulier & $\{\mathrm{F} 93 \times 3\}$ \\
\hline 4. Gain de poids & $\{\mathrm{F} 93 \times 4\}$ \\
\hline 5. Infécondité & $\{F 93 \times 5\}$ \\
\hline 6. Aménorrhée (absence de règles) & $\{F 93 \times 6\}$ \\
\hline$x_{0}^{2}$ & $\{F 93 \times 7\}$ \\
\hline 77. Aucun problème mentionné & \{F93X77\} \\
\hline 98. Ne sait pas & \{F93X98\} \\
\hline
\end{tabular}


94. En dehors des visites régulières de contrôle, quels sont les problèmes, s'il y en a, pour lesquels vous pensez qu'une femme recevant les injections devrait revenir dans la structure sanitaire? (Ne pas lire la liste, mais suscitez davantage de réponses en demandant, "Autres problèmes?")

\begin{tabular}{|c|c|}
\hline & Cochez tous ceux qui sont mentionnés \\
\hline 1. Maux de tête (graves, persistants) & \{F94X1\} \\
\hline 2. Saignement abondant & $\{\mathrm{F} 94 \times 2\}$ \\
\hline 3. Douleur thoracique aiguë, problèmes de respiration & $\{\mathrm{F} 94 \times 3\}$ \\
\hline 4. Douleur aiguë au bas ventre & $\{F 94 \times 4\}$ \\
\hline 5. Douleur aiguë dans les jambes & $\{\mathrm{F} 94 \times 5\}$ \\
\hline 6. Baisse de la vision ou vision floue & $\{\mathrm{F} 94 \mathrm{X} 6\}$ \\
\hline 7. Urines fréquentes & $\{\mathrm{F} 94 \times 7\}$ \\
\hline 8. Retard des règles & \{F94X8\} \\
\hline $194 \times 9$ & \{F94X9\} \\
\hline 77. Aucun problème mentionné & $\{\mathrm{F} 94 \times 77\}$ \\
\hline 98. Ne sait pas & $\{\mathrm{F94X98 \}}$ \\
\hline
\end{tabular}

95. L'injection protège-t-elle la femme contre les MSTNIH?

$1=$ Oui

2 = Partiellement

$3=$ Non

$98=$ Ne sait pas

96. Passez à la q.101 


\section{POUR LES UTILISATRICES DE PRÉSERVATIFS ET DE SPERMICIDES}

97. Les préservatifs/spermicides protègent-ils la femme contre les MST/NIH ? $\quad$ [F97\}

$$
\begin{aligned}
& 1=\text { Oui } \\
& 2=\text { Partiellement } \\
& 3=\text { Non } \\
& 98=\text { Ne sait pas }
\end{aligned}
$$

98. Avez-vous reçu des provisions de préservatifs et/ou spermicides (crème, mousse, gelée) aujourd'hui ? $\{\mathrm{F98}\}$

1 = Oui (passez à la q.100)

$2=$ Non

99. (Si non) Où irez-vous pour vous approvisionner en préservatifs et/ou spermicides (crème, mousse, gelée) ? (Encerclez une.) \{F99\}

1 = Dans une autre section de ce même PPS aujourd'hui (passez à la q. 101\}

2 = Reviendra dans ce PPS une autre fois (passez à la q. 101\}

$3=$ Un autre PPS (passez à la q. 101\}

4 = Pharmacie / boutique / pharmacien / médecin privé (passez à la q. 101\}

5 = DBC (passez à la q. 101\}

6. = Autre:

(passez à la q. 101) $\quad$ \{F90R\}

98 = Ne sait pas (passez à la q. 101\}

100. Quand vous épuiserez cette provision de préservatifs et/ou spermicides, où irez-vous pour votre réapprovisionnement? (Encerclez la réponse qui s'applique.) \{F100\}

1 = Dans cette structure sanitaire

2 = Dans une autre structure sanitaire

3 = Pharmacie / boutique / pharmacien / médecin privé

$4=\mathrm{DBC}$

5. = Autre:

\{F100R\}

$98=$ Ne sait pas 


\section{POUR TOUTES LES CLIENTES}

101. Maintenant, je voudrais vous poser quelques questions sur vous-même. Allaitez-vous actuellement ? \{F101\}

$$
\begin{aligned}
& 1=\text { Oui } \\
& 2=\text { Non (passez à la q.103) }
\end{aligned}
$$

102. (Si oui) Allaitez-vous exclusivement, presque exclusivement, ou complétez-vous régulièrement avec des aliments et/ou des jus? $\{$ F102\}

1 = Allaite exclusivement

2 = Presque exclusivement

3 = Compléte régulièrement avec des aliments et/ou des jus

98 = L'interviewer est incapable de déterminer le degré de suppléments d'aliments

103. Quel âge avez-vous ? \{103\}

$$
\text { ans }
$$

98 = Ne sait pas

104. Quelle est votre situation matrimoniale ? (Insistez pour savoir la situation matrimoniale exacte.) [F104\}

1 = Mariée / monogame (passez à la q.106)

2 = Mariée / polygame (passez à la q.106)

3 = Cohabite (passez à la q.106)

4 = Célibataire, jamais mariée

5 = Divorcée / séparée / veuve

105. (Si pas mariée) Avez-vous un partenaire régulier? \{F105\}

$1=$ Oui

2 = Non (passez à la q.108)

106. Avez-vous jamais discuté de la planification familiale avec votre mari ou votre partenaire régulier? $\{\mathrm{F} 106\}$

$$
\begin{aligned}
& 1=\text { Oui } \\
& 2=\text { Non } \\
& 98=\text { Ne sait pas }
\end{aligned}
$$

107. Votre mari ou votre partenaire régulier sait-il que vous utilisez ou prévoyez d'utiliser la planification familiale? \{F107\}

$$
\begin{aligned}
& 1=\text { Oui } \\
& 2=\text { Non } \\
& 98=\text { Ne sait pas }
\end{aligned}
$$


108. Quel est le nombre de vos propres enfants vivants? $\{F 108\}$

$$
\text { enfants }
$$

(Si aucun, passez à la q.110)

109. Quel est l'âge de votre dernier né ? \{F109\}

$$
\text { mois }
$$

98 = Ne sait pas

110. Aimeriez-vous avoir un (davantage) d'enfant(s) ? (Encerclez une.) $\{\mathrm{F} 110\}$

$1=$ Oui

2 = Non (passez à la q.112)

3 = Dépend du mari (passez à la q.112)

4 = Dépend de Dieu (passez à la q.112)

5 = N'ait pas sure / indécise (passez à la q.112)

111. (Si oui) Quand souhaitez-vous avoir (un) (le prochain) enfant ? (Encerclez une.) \{F111\}

$1=$ Dans moins d'un an

$2=$ Dans un an

$3=$ Entre un et deux ans

$4=$ Deux ans

$5=$ Plus de deux ans

6 = Toute autre réponse

$98=$ Ne sait pas

112. Pouvez-vous lire et comprendre aisément, avec difficulté ou pas du tout une lettre ou un journal ? $\{\mathrm{F} 112\}$

1 = Aisément

2 = Avec difficulté

$3=$ Pas du tout

113. Quelles langues pouvez-vous lire et comprendre ? (Ne pas lire la liste, mais suscitez davantage de

\begin{tabular}{|c|c|c|}
\hline & & Cochez toutes celles qui sont mentionnées \\
\hline 1. Anglais/Français & & \{F113X1\} \\
\hline 2. Dialecte local & & $\{\mathrm{F} 113 \times 2\}$ \\
\hline 3. Autre : & $\{\mathrm{F} 113 \times 3 \mathrm{R}\}$ & $\{F 113 \times 3\}$ \\
\hline 4. Autre: & $\{\mathrm{F} 113 \mathrm{X} 4 \mathrm{R}\}$ & $\{F 113 \times 4\}$ \\
\hline 5. Autre: & \{F113X5R\} & $\{\mathrm{F} 113 \times 5\}$ \\
\hline
\end{tabular}
réponses en demandant, "Autres langues ?") 
114. Quel est votre niveau scolaire? (Encerclez une.) $\{F 114\}$

$$
\begin{aligned}
& 1 \text { = N'a pas été à l'école } \\
& 2 \text { = Primaire } \\
& 3 \text { = Fondamentale } \\
& 4=\text { Secondaire } \\
& 5=\text { Supérieur } \\
& 6=\text { Post-universitaire }
\end{aligned}
$$

115. Quelle est votre religion? (Encerclez une.)

$$
\begin{aligned}
& 1 \text { = Protestante } \\
& 2 \text { = Catholique } \\
& 3=\text { Musulmane } \\
& 4=\text { Animiste } \\
& 5=\text { Aucune } \\
& 6 \text {. = Autre : }
\end{aligned}
$$

116. Pour terminer, je voudrais vous poser quelques questions sur d'autres problèmes de santé de la reproduction. J'aimerais vous rappeler que l'information que vous me donnerez restera strictement confidentielle et que vous n'êtes pas obligée de répondre à une question si vous n'en avez pas envie. Une femme peut tomber en état de grossesse à un moment où elle n'est pas prête pour une grossesse. Dans le passé, avez-vous jamais été en état de grossesse à un moment où vous n'étiez pas prête pour une grossesse ? $\{\mathrm{F} 116\}$

$$
\begin{aligned}
& 1 \text { = Oui } \\
& 2=\text { Non (passez à la q.118) } \\
& 98=\text { Ne sait pas / pas de réponse (passez à la q.118) }
\end{aligned}
$$

117. (Si oui) Qu'aviez-vous fait la dernière fois que cela vous est arrivé ? (Encerclez une.) \{F117\}

1 = Rien, a porté la grossesse à terme

2 = A essayé de mettre fin à la grossesse mais sans succès, a accouché

3 = A essayé et réussi à mettre fin à la grossesse

4. = Autre : [F117R]

98 = Ne sait pas / pas de réponse

118. A votre connaissance, y a-t-il une maladie qui peut être transmise par les rapports sexuels ? $\{\mathrm{F} 118\}$

$$
\begin{aligned}
& 1 \text { = Oui } \\
& 2=\text { Non (passez à la q.120) } \\
& 98=\text { Ne sait pas (passez à la q.120) }
\end{aligned}
$$


119. A partir de ce que vous avez entendu ou lu, quels sont quelques uns des signes et symptômes courants des maladies sexuellement transmissibles? (Ne pas lire la liste, mais suscitez davantage de réponses en demandant, "Autres signes ou symptômes?")

\begin{tabular}{|l|l|}
\hline & Cochez tous ceux qui sont mentionnés \\
\hline 1. Pertes vaginales anormales & $\{\mathrm{F} 119 \mathrm{X} 1\}$ \\
\hline 2. Saignement vaginal anormal & $\{\mathrm{F} 119 \mathrm{X} 2\}$ \\
\hline 3. Démangeaison de l'appareil génital & $\{\mathrm{F} 119 \mathrm{X} 3\}$ \\
\hline 4. Lésions / plaies & $\{\mathrm{F} 119 \mathrm{X} 4\}$ \\
\hline 5. Douleur au bas ventre & $\{\mathrm{F} 119 \mathrm{X} 5\}$ \\
\hline 6. Douleur pendant les rapports sexuels & $\{\mathrm{F} 119 \mathrm{X} 6\}$ \\
\hline 7. Douleur pendant l'urine & $\{\mathrm{F} 119 \mathrm{X} 7\}$ \\
\hline 8. Croissance anormale au niveau de l'appareil génital (verrues) & $\{\mathrm{F} 119 \mathrm{X} 8\}$ \\
\hline 9. Ecoulement urétral & $\{\mathrm{F} 119 \mathrm{X} 9\}$ \\
\hline 10. Perte de poids & $\{\mathrm{F} 119 \mathrm{X} 10\}$ \\
\hline 11. Diarrhée de longue durée & $\{\mathrm{F} 119 \mathrm{X} 11\}$ \\
\hline 12. Autre: & $\{\mathrm{F} 119 \mathrm{X} 12\}$ \\
\hline 98. Ne sait pas & $\{\mathrm{F} 119 \mathrm{X} 98\}$ \\
\hline
\end{tabular}

120. Avez-vous déjà entendu parler du VIH ou du SIDA, ou bien n'êtes vous pas sûre ? \{F120\}

$$
\begin{aligned}
& 1 \text { = Oui } \\
& 2 \text { = Non (passez à la q.122) } \\
& 98 \text { = N'est pas sare (passez à la q.122) }
\end{aligned}
$$

\begin{tabular}{|c|c|}
\hline & Cochez tous ceux qui sont mentionnés \\
\hline 1. Rapports sexuels & $\{\mathrm{F} 121 \times 1\}$ \\
\hline 2. Transfusion sanguine & $\{\mathrm{F} 121 \times 2\}$ \\
\hline 3. Le fait de partager les objets tels que lames de rasoir ou aiguilles & $\{\mathrm{F} 121 \times 3\}$ \\
\hline 4. De mère à l'enfant & $\{F 121 \times 4\}$ \\
\hline 5. Autre: & $\{\mathrm{F} 121 \times 5\}$ \\
\hline 98. Ne sait pas & [F121X98\} \\
\hline
\end{tabular}

121. A votre connaissance, quels sont les moyens par lesquels on contracte le VIH/SIDA ? (Ne pas lire la liste mais suscitez davantage de réponses en demandant, "Autres moyens ?") 
122. Connaissez-vous des moyens par lesquels vous pouvez vous protéger contre les MST y compris le $\mathrm{VHI} / \mathrm{SIDA} ? \quad\{\mathrm{~F} 122\}$

$1=$ Oui

$2=$ Non (passez à la q.124)

123. (Si oui) Quels sont ces moyens pour vous protéger? (Ne pas lire la liste mais suscitez davantage de réponses en demandant, "Autres moyens ?")

\begin{tabular}{|c|c|}
\hline & Cochez tous ceux qui sont mentionnés \\
\hline 1. Rester fidèle à votre époux/partenaire unique & $\{\mathrm{F} 123 \times 1\}$ \\
\hline 2. Encourager l'époux/partenaire à rester fidèle & $\{\mathrm{F} 123 \times 2\}$ \\
\hline 3. Utiliser les préservatifs & $\{\mathrm{F} 123 \times 3\}$ \\
\hline 4. Eviter de partager les aiguilles, rasoirs, etc. & $\{F 123 \times 4\}$ \\
\hline 5. Autre: & $\{F 123 \times 5\}$ \\
\hline
\end{tabular}

124. Combien de partenaires sexuels avez-vous eus au cours des 12 derniers mois ? $\{\mathrm{F} 124\}$ partenaires

(Si aucun, passez à la conclusion)

125. Avez-vous eu des rapports sexuels avec un nouveau partenaire au cours des 12 derniers mois? $\{\mathrm{F} 125\}$

1 = Oui

2 = Non (passez à la conclusion)

98 = Ne sait pas (passez à la conclusion)

126. (Si oui). Aviez-vous utilisé un préservatif la première fois que vous avez eu des rapports sexuels avec votre nouveau partenaire le plus récent?

$$
\begin{aligned}
& 1=\text { Oui } \\
& 2=\text { Non } \\
& 98=\text { Ne sait pas /ne se rappelle pas }
\end{aligned}
$$

Lire la conclusion :

Merci beaucoup pour le temps que vous avez bien voulu consacrer à répondre à nos questions. 
Interview de sortie de la cliente de planification familiale : guide question par question

La cliente vient juste de recevoir les services de PF. Très probablement, une infirmière/sage-femme de l'équipe de recherche sur le terrain a terminé l'observation des services qu'elle a reçus et vous a apporté maintenant la cliente. Soyez sensible au fait que la cliente peut être venue de loin, qu'elle a attendu longtemps les services, et peut avoir subi une rude épreuve. Cependant, elle peut accepter une interview avec une personne sensible et intéressante. (Pour plus d'informations, voir la section "Astuces pour réaliser une bonne interview" à la page 22.)

Il faut conduire la cliente dans un endroit privé pré-choisi, loin du personnel clinique et des autres clientes et ensuite lire les salutations. Ces salutations sont extrêmement importantes parce qu'elles assurent que la cliente a donné son "consentement éclairé" pour participer à l'étude. Demandez à la cliente si elle a des questions à poser sur l'étude. Si nécessaire, vous pouvez encourager un peu la cliente en lui disant, "Votre participation sera d'une très grande aide," mais dans tous les cas vous devez respecter la volonté de la cliente si elle ne veut pas être interviewée. Si elle refuse de participer, il faut la remercier et passer à la cliente suivante.

Vous devriez remplir les informations des deux premières pages avant de commencer l'interview. Si vous avez des doutes sur l'un des codes, demandez à votre Chef d'équipe. Ecrivez (ne pas signer) votre nom dans l'encadré en bas de la page. Lorsque vous finissez l'interview, votre Chef d'équipe la révisera et signera en bas de votre nom.

En ce qui concerne le reste de l'interview, suivez les instructions pour enregistrer les réponses de la cliente en encerclant une réponse précodée, en cochant un encadré ou en écrivant une réponse comme demandé. Certaines questions sont des questions filtres, c'est-à-dire qu'elles classent les clientes qui ont eu une certaine expérience par rapport à celles qui n'ont pas eu cette expérience. Généralement, les clientes qui ont eu l'expérience, telle que la pose d'un DIU, seront soumises à certaines questions auxquelles les autres clientes n'auront pas à répondre. Ces questions filtres incluent toujours des "exemples de saut" qui permettent le saut de ces questions pour les clientes qui n'ont pas eu l'expérience. Faites très attention à tous les exemples de saut. $\mathrm{A}$ la fin de l'interview, révisez vite vos réponses codées pour voir si elles sont au complet et correctes, et vous assurer que vous avez suivi les sauts correctement. Si possible, corrigez toutes les erreurs pendant que la cliente est encore disponible.

\section{Pour toutes les clientes}

1. Dans l'ensemble, êtes-vous satisfaite de votre visite aujourd'hui dans cette structure sanitaire ou n'êtes-vous pas satisfaite d'elle?

Souvent les clientes hésitent à exprimer tout mécontentement général parce qu'elles veulent être courtoises. Cependant elles ont tendance à exprimer plus leur mécontentement avec des questions plus spécifiques. Les questions 7 à 17 de la présente interview donneront à la cliente plus d'opportunité de critiquer spécifiquement les services.

2. Pourquoi n'êtes-vous pas satisfaite de votre visite d'aujourd'hui ?

Nous nous intéressons beaucoup aux raisons de la non satisfaction. Ne vous précipitez pas. A l'aide d'un petit sondage, vous pouvez encourager la cliente à parler davantage du mécontentement qu'elle a mentionné. Si nécessaire, utilisez les sondages du genre "Pouvez-vous me dire davantage pourquoi vous avez un tel sentiment ?"

3. En plus de la planification familiale, avezvous reçu d'autres services de santé de la part du prestataire?
4. A présent j'aimerais vous poser des questions sur tous les services que vous 
avez reçus aujourd'hui. Avez-vous reçu l'un des services suivants ?

Ces questions traitent de l'intégration d'autres services avec la planification familiale. Si les clientes disent qu'elles ont reçu d'autres services à la question 3, posez la question 4 (voir l'exemple du saut). Dans la question 4, lisez chacun des services et placez attentivement une coche (signe de contrôle) dans la colonne de droite et sur la ligne appropriée de chaque service reçu. Rappelezvous qu'une ligne vierge dans la colonne (pas de coche) signifie que la cliente n'a pas reçu ce service aujourd'hui.

5. Avez-vous le sentiment qu'aujourd'hui vous avez reçu l'information et les services que vous vouliez?

Les clientes se rendent dans un PPS pour obtenir des informations et des services particuliers. L'objet de cette question est de voir si la cliente a reçu l'information et les services qu'elle désirait.

6. Pensez-vous que votre consultation de PF avec l'agent de santé a été trop courte, trop longue, ou juste le temps qu'il faut? Cette information peut être comparée plus tard avec la durée réelle de la consultation (à partir de l'observation) pour trouver la durée du temps avec lequel les clientes se sentent plus à l'aise.

7. Pendant la visite, aviez-vous des inquiétudes par rapport à la PF ou d'autres questions de santé que vous vouliez discuter avec le prestataire?

8. (Si oui) Le prestataire a-t-il écouté vos préoccupations jusqu'à ce que vous ayez satisfaction?

Le terme "inquiétudes" est très vaste. Les inquiétudes incluent mais ne se limitent pas aux soucis ou aux rumeurs des risques pour la santé, aux effets secondaires, à l'intimité, aux réactions du partenaire, etc. Si la cliente dit qu'en réalité elle avait des inquiétudes, posez-lui la question 8 .

9. Pendant cette visite, aviez-vous des questions que vous vouliez poser?

10. (Si oui) Le prestataire vous a-t-il donné le temps de les poser?

11. (Si oui) Etes-vous satisfaite des réponses que le prestataire a données à vos questions?

Le fait que la cliente soit libre de poser des questions et qu'elle soit satisfaite des réponses données est un bon indicateur de la qualité des relations interpersonnelles, et probablement a un impact sur la continuité de la cliente à utiliser la PF. Ces trois questions cherchent à savoir si la cliente se sentait à l'aise en demandant l'information dont elle avait besoin.

"Les questions" peuvent porter sur n'importe quel sujet tel que le mode d'octroi d'une méthode ou l'endroit pour acheter des préservatifs. Les questions peuvent aussi porter sur des "inquiétudes" comme défini cidessus.

La cliente peut tenir compte de plusieurs aspects en répondant à la question 11 : par exemple, le caractère complet, l'exactitude ou la manière que le prestataire a utilisé dans ses réponses. N'assistez pas la cliente à répondre à cette question parce qu'elle est la seule qui puisse déterminer sa propre satisfaction avec les réponses à ses questions.

12. Pendant cette visite, le prestataire a-t-il fait des examens ou des procédures de santé ?

13. (Si oui) Le prestataire a-t-il expliqué les examens ou procédures avant de les faire?

14. Le prestataire a-t-il expliqué les résultats des examens ou procédures de santé ? "Les examens de santé" incluent les examens de la tête jusqu'aux pieds, les examens mammaires, les examens pelviens, et l'examen de toute autre partie du corps. "Les 
procédures" incluent la prise de la tension artérielle ou de la température, la pose d'un DIU, une injection, etc.

L'idée qui sous-tend ces trois questions est que si les clientes connaissent les examens et les procédures, elles seront plus à l'aise pendant leur visite. Une explication de l'examen ou de la procédure implique le fait de dire à la cliente ce que le prestataire s'apprête à faire, pourquoi il le fait, et ce que la cliente peut sentir. L'explication des résultats de l'examen ou de la procédure implique le fait de dire à la cliente si l'infirmière/sage-femme est arrivée à une conclusion ou un diagnostic à partir de l'examen. Si un diagnostic n'est pas disponible immédiatement, comme c'est le cas avec le test de Papanicolaou, alors l'explication des résultats inclurait le fait de dire à la cliente que son prélèvement sera testé et qu'elle sera contactée plus tard pour les résultats.

15. A votre avis, y avait-il assez d'intimité pendant votre consultation avec le prestataire de services?

Il s'agit là de l'avis de la cliente, de ce fait, c'est elle qui le détermine.

16. Au cours de la consultation, avez-vous eu le sentiment que le prestataire était facile à comprendre lorsqu'il vous expliquait certaines choses, ou bien était-il difficile à comprendre?

Il s'agit là de l'avis de la cliente, de ce fait, c'est elle qui le détermine.

17. Si vous aviez une amélioration à suggérer par rapport aux services fournis, laquelle alliez-vous suggérer?

Cette importante question permet à la cliente de mentionner quelque chose qui l'a gênée dans cette expérience, sans être trop grave. Sondez avec des commentaires de soutien tels que : "Tout ce que vous voulez suggérer est bon."
18. Au cours de cette visite, vous a-t-on donné ou avez-vous pris un dépliant ou un matériel éducatif pour l'amener à la maison?

19. (Si oui) Quel est le thème de ce matériel ?

Aucune distinction n'est faite entre dépliants, brochures, prospectus ou tout autre matériel éducatif. Essayez de trouver le thème du dépliant ou du matériel éducatif donné à la cliente. Ne lisez pas la liste, mais suscitez davantage de réponses en demandant "Y a-til d'autres thèmes?"

20. Avez-vous pris part à une causerie de groupe dans cette structure sanitaire aujourd'hui?

La "causerie de groupe" est souvent appelée "causerie sanitaire." Elle est généralement dirigée par une infirmière/sage-femme, et peut inclure plusieurs thèmes. Elle est fréquemment sous forme de cours magistral, mais lorsqu'elle est bien faite, elle peut inclure des questions, des expositions d'échantillons ou autres supports audiovisuels. "Prendre part" et "entendre" sont synonymes dans cette question.

21. (Si oui) Quels sont les thèmes qui ont été abordés pendant cette causerie de groupe?

Ne posez cette question que lorsque la cliente a pris part à une causerie de groupe. Nous sommes intéressés par l'information dont la cliente se rappelle pendant la causerie sanitaire, donc, il ne faut pas lui souffler en lisant la liste des thèmes. Il faut remarquer aussi le sondage approprié, "Autres thèmes?"

22. Un prestataire de services vous a-t-il dit quand est-ce qu'il faut revenir pour une autre visite?

Cette information est aussi recueillie dans l'observation, cette question peut donc tester 
si les clientes auxquelles on a donné des dates de suivi connaissent ces dates.

23. Les heures d'ouverture de ce PPS sontelles convenables pour vous ?

24. (Si non) Quel est le temps qui vous conviendrait le mieux?

Les heures d'ouverture des services peuvent être une grande barrière pour les clientes, et ces questions portent sur la convenance des horaires de la clinique. Même dans les contextes où les horaires de la clinique sont fixés par le ministère de la Santé ou par une autorité centrale, les informations issues de ces questions peuvent être très utiles pour obtenir des changements de ces politiques si nécessaire.

25. Avez-vous jamais été renvoyée de cette structure sanitaire pendant les heures officielles de travail ?

"Renvoyée" veut dire qu'on a refusé de lui fournir les services ou bien on lui a dit de revenir une autre fois. Remarquez qu'il s'agit là de l'antécédent de la cliente avec le PPS et non pas de la visite d'aujourd'hui. Ceci implique aussi le renvoi à cause du fait qu'il y a trop de clientes ou autre raison non spécifiée. Si la cliente est venue une seule fois, a rencontré un prestataire qui lui a dit de revenir lorsqu'elle verra ses règles, elle n'est pas comptée comme "renvoyée" pour cette question.

26. Environ combien de temps vous a-t-il fallu attendre entre le moment où vous êtes arrivée à ce PPS et celui auquel vous avez commencé à recevoir les services pour lesquels vous êtes venue?

Le but de cette question est de déterminer le temps d'attente de la cliente. Encouragez la cliente à faire une estimation du temps pendant lequel elle a attendu si elle n'a pas de montre et/ou si elle n'a pas relevé le temps. Réservez la mention "ne sait pas" pour les clientes qui ne sont pas en mesure de faire une estimation même si on leur demande de le faire.

27. Avez-vous le sentiment que votre temps d'attente était raisonnable ou trop long ?

Il s'agit là de l'opinion de la cliente sur l'ensemble du temps d'attente qu'elle a fait avant de commencer à recevoir les services pour lesquels elle était venue. Il ne faut pas considérer le début des procédures d'inscription comme étant les services pour lesquels elle est venue.

28. Combien de temps vous a-t-il fallu pour arriver ici aujourd'hui ?

Nous nous informons sur le temps de voyage de la cliente depuis son domicile parce que le temps de voyage peut constituer parfois une grande barrière aux services. Si la cliente est venue de son lieu de travail ou de chez un parent, il faut calculer le temps à partir de làbas. Encouragez la cliente à estimer le temps approximatif en minutes.

29. Quel a été le principal moyen de transport que vous avez utilisé pour venir ici ? La cliente peut avoir utilisé deux ou trois, voire plusieurs moyens de transport-la marche et le minibus seraient une combinaison courante. Lorsque la cliente utilise deux ou plusieurs moyens de transport, il faut l'encourager à décider lequel est le principal moyen. Tout critère qu'elle utilise pour décider-temps, distance, coût, etc.est bon.

30. A votre connaissance, quels types de services autres que la planification familiale sont généralement fournis dans cette structure sanitaire?

Cette question traite de la connaissance d'autres services par la cliente, ce qui sera comparée plus tard avec les services 
réellement disponibles. Cela permet de savoir si certains services sont sous utilisés simplement à cause du fait que les gens ne les connaissent pas.

Sachez que la cliente n'utilisera pas nos termes exacts. Faites de votre mieux pour interprétez ses commentaires selon les catégories. Si la cliente dit "MST" ou "VIH," vous pouvez la sonder pour savoir en posant la question "Quels types de services de MST ou de VIH sont généralement fournis ?" afin de vous rapprocher plus près des catégories de codes disponibles.

31. En dehors de ce PPS, y a-t-il un autre endroit près de chez vous où vous pouvez obtenir des services de planification familiale?

32. (Si oui) Quel est le type de cette structure sanitaiare?

33. Quelle serait la principale raison pour laquelle vous n'étiez pas allée solliciter des services PF là-bas?

Le choix d'une cliente pour un PPS est important parce qu'il peut être motivé par la qualité des services qu'elle a reçus. Ces trois questions portent sur les clientes qui ont choisi de venir dans ce PPS au lieu d'un autre.

Si la cliente dit qu'il y a un autre PPS près de son domicile ou de son lieu de travail, ou une autre place qu'elle visite régulièrement, il faut coder la question 31 en conséquence. Ensuite les questions 32 et 33 devraient être posées, et la cliente encouragée à penser à un seul PPS, celui qui est le plus proche de sa maison ou de son lieu de travail. A la question 33, la cliente peut mentionner plus d'une raison. Encouragez-la à décider pour une seule raison.

34. A présent, j'aimerais vous poser des questions sur le coût de votre transport et les services que vous avez reçus dans cette clinique. Combien avez-vous payé pour votre consultation?
35. Combien avez-vous payé pour les médicaments ?

36. Combien avez-vous payé pour les autres produits tels que le coton, les gants, les produits antiseptiques ou les seringues?

37. Combien avez-vous payé pour la carte d'inscription/de membre ?

38. Combien avez-vous payé pour le transport ?

39. Combien avez-vous payé pour tout autre service ou frais?

Dans l'inventaire, la structure sanitaire indiquera en détails combien elle demande aux clientes PF pour leurs services.

L'information relative au montant réel payé par les clientes fera ressortir le vrai coût des services que les individus payent (y compris le transport, etc.) par rapport au prix perçu par la structure sanitaire.

Les clientes peuvent connaître le total payé sans savoir comment les charges ont été reparties. Si c'est le cas, faites une note à la marge avec le montant total.

40. Dans l'ensemble, pensez-vous que le coût total pour obtenir les services est beaucoup trop cher, un peu trop cher ou acceptable pour vous?

Puisque le fardeau des dépenses ne peut être jugé que par rapport au revenu de la cliente, cette question donne à la cliente l'occasion d'évaluer les difficultés qu'elle éprouve pour payer les services, même si le coût est assez bas.

41. Si je comprends bien, la principale raison de votre visite dans cette structure sanitaire aujourd'hui était (insérer la raison ). Est-ce exact ?

Il s'agit là d'une question filtre pour déterminer à quel endroit du questionnaire vous devez allez ensuite. En fonction de la principale raison de la visite de la cliente, nous posons différentes questions. Il est donc très important d'identifier correctement la principale raison pour laquelle la cliente s'est 
rendue à la clinique. Regardez à la page 66 pour voir la principale raison de la visite de la cliente. Ensuite, lisez la question 41 pour vérifier si la principale raison a été enregistrée correctement. Si c'est fait, encerclez alors la raison dans la question 41 et passez à la question appropriée. Si non, demandez alors à la cliente la vraie raison de sa visite, encerclez sa réponse et passez ensuite au numéro de la question appropriée.

\section{Pour les nouvelles acceptantes}

42. Juste pour me rassurer, je comprends que vous n'utilisiez pas de méthode contraceptive avant de venir aujourd'hui dans cette structure sanitaire. Est-ce exact que vous n'utilisiez pas de méthode contraceptive?

Le but de cette question est de vérifier une dernière fois pour voir si nous avons classé la cliente correctement avant de poser d'autres questions.

43. Avez-vous déjà utilisé une méthode contraceptive dans le passé ?

44. (Si oui) Quelle(s) méthode(s) avez-vous déjà utilisée(s) ?

Les méthodes utilisées par le mari/partenaire(s) telles que les préservatifs, la vasectomie et le retrait sont aussi attribuées aux clientes.

\section{Avez-vous reçu une méthode contraceptive} au cours de cette visite?

Cette question porte sur la méthode que la cliente a décidé d'utiliser aujourd'hui. Si elle a décidé d'utiliser l'injectable mais ne l'a pas reçu aujourd'hui, il faut la compter comme "oui." De même, si elle a choisi la MAMA ou la PF naturelle, ce qui n'implique pas une réception de quelque chose, il faut la compter comme "Oui."
46. ( $\mathrm{Si}$ non) Quelle est la raison principale pour laquelle vous n'avez pas obtenu de méthode contraceptive aujourd'hui ? S'il y a plus d'une raison, faites un sondage pour savoir la principale raison.

47. Quelle(s) méthode(s) avez-vous acceptée(s) aujourd'hui ?

Tout comme la question 45 , celle-ci porte sur la méthode que la cliente a décidé d'utiliser aujourd'hui. Si elle a décidé d'utiliser les injectables mais n'a pas reçu d'injection aujourd'hui, cochez injectable. De même, si elle a choisi la MAMA ou la PF naturelle, ce qui n'implique pas une réception physique de quelque chose, il faut cocher ces méthodes.

48. Le prestataire vous a-t-il parlé d'une autre méthode?

49. Le prestataire vous a-t-il dit que vous pouvez changer de méthodes si vous n'êtes pas satisfaite de celle-ci ?

50. Le prestataire vous a-t-il indiqué une autre source d'approvisionnement ou de suivi pour votre méthode?

L'observation enregistrera si tous ces trois éléments ont eu lieu. Ces questions sont posées encore dans l'interview de sortie, et les résultats seront comparés avec l'observation. Ceci permettra de savoir si l'information a été communiquée à la cliente de manière efficace.

51. Y a-t-il une autre méthode différente de celle que vous avez acceptée aujourd'hui et que vous préfériez utiliser?

52. (Si oui) Quelle méthode préfériez-vous ? Les travaux de recherche semblent indiquer une faible continuation/satisfaction si les clientes n'obtiennent pas leur méthode préférée, indépendamment de l'efficacité technique de la méthode. Ainsi, nous nous intéressons beaucoup au fait de savoir si elles ont reçu leur méthode préférée, et quelle est 
cette méthode. En plus cette information sera enregistrée dans l'observation, et ces résultats pourront être comparés avec ceux de l'observation. Rappelez-vous de ne poser la question 52 que lorsque la cliente répond "oui" à la question 51 .

53. Pourquoi n'allez-vous pas utiliser cette méthode préférée?

Cette réponse sera enregistrée aussi dans l'observation, ce qui permettra de savoir, par exemple, si les clientes qui ont des contreindications pour une méthode savent que c'est ça la raison pour ne pas utiliser cette méthode.

54. A présent, je voudrais vous demander de m'expliquer comment utiliser (lire la méthode).

Au cas où la cliente accepte deux ou des méthodes multiples-telles que les préservatifs et les spermicides, ou les pilules et les préservatifs-on vous demande d'encercler la méthode la plus efficace acceptée et de ne poser la question que sur cette méthode.

\section{Pour les anciennes clientes qui n'ont pas de} problèmes

55. Juste pour me rassurer, je comprends que vous utilisiez une méthode contraceptive avant de venir dans cette structure sanitaire aujourd'hui. Est-ce correct que vous utilisiez une méthode?

Le but de cette question est de faire un dernier contrôle pour voir si nous avons classé la cliente correctement pour les questions suivantes.

56. Quelle(s) méthode(s) utilisiez-vous ? Les méthodes utilisées par le mari/ partenaire(s) telles que les préservatifs, la vasectomie et le retrait sont aussi attribuées à la cliente.
57. Avez-vous l'intention de continuer à utiliser cette méthode ?

Notez que si la cliente ne va pas continuer à utiliser cette méthode, elle est alors traitée comme une ancienne qui a un problème et vous devez passer à la section suivante, et la question de savoir pourquoi elle a décidé d'arrêter ou de changer de méthode.

58. Je voudrais vous demander maintenant comment utiliser (lire la méthode). $\mathrm{Au}$ cas où les clientes acceptent des méthodes doubles ou multiples-telles que les préservatifs et le spermicide, ou les pilules et les préservatifs-on vous demande d'encercler la méthode la plus efficace acceptée et de ne poser la question que sur cette méthode.

Pour les anciennes clientes qui ont des problèmes avec la méthode, qui veulent changer ou arrêter

59. Juste pour me rassurer, je comprends que vous utilisiez une méthode contraceptive avant de venir dans cette structure sanitaire aujourd'hui. Est-ce exact que vous utilisiez une méthode?

Le but de cette question est de faire un dernier contrôle pour voir si nous avons classé la cliente correctement pour les questions suivantes.

60. Quelle(s) méthode(s) utilisiez-vous ? Les méthodes utilisées par le mari/ partenaire(s) telles que les préservatifs, la vasectomie et le retrait sont aussi attribuées à la cliente.

61. Est-ce vrai que vous aviez un problème, que vous vouliez changer de méthodes ou arrêter d'utiliser la planification familiale ? Cette question permet de vérifier que la cliente est en réalité une ancienne cliente qui a eu des problèmes. Si non, elle est alors traitée comme une ancienne cliente normale et transférée dans la section appropriée. 
62. Quel est le problème majeur que vous aviez, ou la principale raison pour laquelle vous vouliez changer ou arrêter l'utilisation de la PF ?

S'il y a plus d'une raison, encouragez la cliente à choisir la principale raison.

63. (Si avait des effets secondaires médicaux) Quand vous avez décidé pour la première fois d'utiliser cette méthode, vous avait-on dit que ce problème pourrait arriver ?

64. (Si oui) Vous avait-on dit ce qu'il fallait faire de ce problème?

Si une cliente accepte une méthode et qu'on lui explique les effets secondaires de cette méthode et comment il faut les gérer, alors elle a de fortes chances à continuer l'utilisation de cette méthode si les effets secondaires se manifestent. Cette question permet de savoir si les clientes qui ont eu des effets en avaient été informées auparavant, même si cette information ne provenait pas de ce PPS.

65. Après cette consultation aujourd'hui, avezvous le sentiment que vous recevez l'assistance appropriée pour vos besoins particuliers ?

Il s'agit là d'une question de satisfaction, spécifiquement à l'intention des clientes qui ont besoin d'une attention particulière pour les problèmes qu'elles rencontrent avec leurs méthodes.

66. Qu'allez-vous faire maintenant en matière de planification familiale?

67. (Si change de méthode) Quelle(s) méthode(s) utiliserez-vous désormais? Ces deux questions permettront d'identifier les clientes qui changent de méthodes. Ces clientes sont importantes parce qu'elles devraient être conseillées et informées comme si elles étaient de nouvelles clientes.

68. Le prestataire vous a-t-il parlé d'autres méthodes?
69. Le prestataire vous a-t-il dit que vous pouvez changer de méthodes si vous n'êtes pas satisfaite de celle-ci ?

70. Le prestataire vous a-t-il indiqué une autre source d'approvisionnement ou de suivi pour votre méthode?

L'observation enregistrera si oui ou non tous ces trois éléments ont eu lieu. Ces questions sont posées encore dans l'interview de sortie, et les résultats seront comparés avec l'observation. Ceci permettra de savoir si l'information a été communiquée à la cliente de manière efficace.

71. Je voudrais vous demander maintenant comment utiliser (lire la méthode).

Au cas où les clientes acceptent des méthodes doubles ou multiples-telles que les préservatifs et le spermicide, ou les pilules et les préservatifs-on vous demande d'encercler la méthode la plus efficace acceptée et de ne poser la question que sur cette méthode.

Pour les utilisatrices de pilules

72. Quand une femme se met sous pilule pour la première fois, à quel moment du cycle menstruel pensez-vous qu'elle devrait commencer la prise ?

73. A quelle fréquence pensez-vous qu'une femme devrait prendre la pilule?

74. Selon vous, si une femme oublie de prendre la pilule un jour, que devrait-elle faire?

75. La pilule protège-t-elle la femme contre les MST/VIH ?

Ces quatre questions sont des questions de connaissance de base de la pilule. Les réponses correctes pour les questions 72,73 et 74 sont " 1 ," et pour la question 75 c'est "3."

76. A votre connaissance, quels sont les problèmes, s'il y en $a$, qu'une femme peut avoir en prenant la pilule?

77. En dehors de la visite régulière de réapprovisionnement, à votre avis quels 
sont les problèmes, s'il y en a, pour lesquels une femme sous pilule devrait revenir au PPS ?

Ces questions sont aussi des questions de connaissance, mais il n'existe pas de réponse "correcte" pour elles, puisqu'il se peut que les clientes sachent n'importe quel nombre de symptômes ou de problèmes. Si la cliente ne mentionne pas de problème, cochez la ligne 98.

Sachez que la cliente peut ne pas utiliser nos termes. Classez de votre mieux ce que la cliente dit, en utilisant nos termes.

78. Le prestataire vous a-t-il donné une provision de pilules aujourd'hui ?

79. (Si non) Où irez-vous vous approvisionner en pilules?

Il est probable que les clientes qui reçoivent des provisions et des informations sur le réapprovisionnement soient mieux disposées à continuer l'utilisation de leur méthode. Cette information sera enregistrée aussi dans l'observation, donc les résultats peuvent être comparés.

80. Allez à la q.101.

Pour les utilisatrices du DIU

81. Votre visite d'aujourd'hui au PPS a-t-elle porté sur la pose, le retrait, ou un suivi de routine du DIU?

Cette question permet de vérifier si la cliente a réellement reçu le DIU aujourd'hui.

82. A votre connaissance, comment une femme peut vérifier pour être sûre que le DIU est en place?

83. A votre connaissance, quels sont les problèmes, s'il y en $a$, qu'une femme peut avoir avec le DIU ?

84. A votre avis, après la pose du DIU, quand une femme devrait-elle revenir pour la première visite de contrôle ?

85. En dehors des visites régulières de contrôle, quels sont les problèmes, s'il y en a, pour lesquels vous pensez qu'une femme portant le DIU devrait revenir au PPS ?

86. Pendant combien d'années pensez-vous qu'une femme peut continuer à utiliser son DIU une fois qu'il est posé?

87. Le DIU protège-t-il la femme contre les MST/VIH ?

Toutes ces questions sont des questions de connaissance générale sur le DIU. Les réponses des clientes seront comparées avec l'information qu'on leur a fournie pendant la consultation et que l'on a notée dans l'observation.

En ce qui concerne les questions 83 et 85 , sachez que la cliente n'utilisera pas nos termes. Classez de votre mieux ce que la cliente dit, en utilisant nos termes. Si la cliente ne mentionne pas de problème, cochez la ligne 98.

88. Allez à la q.101.

\section{Pour les utilisatrices d'injectables}

89. Avez-vous reçu une injection aujourd'hui ? Cette question permet de vérifier si la cliente a réellement reçu une injection aujourd'hui.

90. (Si non) Où irez-vous pour prendre votre injection?

Cette question traite de l'approvisionnement en contraceptifs. Elle a été enregistrée aussi dans l'observation, donc les réponses peuvent être comparées.

91. Quel type d'injection avez-vous reçue ou recevrez-vous?

Le Depo Provera est exigé tous les trois mois et le Noristera tous les deux mois. Si la cliente mentionne le temps au lieu du type, codez correctement. 
92. A votre avis, à quelle fréquence devriezvous prendre cette injection ?

Comme mentionné ci-dessus, le Depo Provera est exigé tous les trois mois et le Noristerat tous les deux mois.

93. A votre connaissance, quels sont les problèmes, s'il y en $a$, qu'une femme peut avoir après avoir reçu une injection ?

94. En dehors des visites régulières de contrôle, quels sont les problèmes, s'il y en $a$, pour lesquels vous pensez qu'une femme recevant les injections devrait revenir au PPS ?

95. L'injection protège-t-elle la femme contre les MST/VIH?

Ces questions sont des questions de connaissance générale pour les utilisatrices des injectables. Remarquez qu'il n'existe pas de réponses strictement "correctes" pour les questions 93 et 94 , puisqu'il se peut que les clientes sachent n'importe quel nombre de symptômes ou de problèmes. Sachez que la cliente n'utilisera pas nos termes. Classez de votre mieux ce que la cliente dit, en utilisant nos termes. Si la cliente ne mentionne pas de problème, cochez la ligne 98.

96. Allez à la q.101.

Pour les utilisatrices de préservatifs et de spermicides

97. Les préservatifs/spermicides protègent-ils la femme contre les MST/VIH ?

C'est la seule question de connaissance pour les utilisatrices du préservatif/ spermicide. Il est particulièrement important que les clientes sachent les vertus protectrices des préservatifs.

98. Avez-vous reçu des provisions de préservatifs et/ou spermicides (crème, mousse, gelée) aujourd'hui ?

99. (Si non) Où irez-vous pour vous approvisionner en préservatifs et/ou spermicides (crème, mousse, gelée) ?
100. Quand vous épuiserez cette provision de préservatifs et/ou spermicides, où irezvous pour votre réapprovisionnement ? Ces questions traitent de l'approvisionnement en contraceptif, ce qui est particulièrement complexe avec les préservatifs puisqu'ils sont souvent disponibles dans beaucoup plus de points que les autres méthodes.

\section{Pour toutes les clientes}

101. Maintenant, je voudrais vous poser quelques questions sur vous-même. Allaitez-vous actuellement?

102. (Si oui) Allaitez-vous exclusivement, presque exclusivement, ou complétez-vous régulièrement avec des aliments et/ou des jus ?

Si la cliente est en train d'allaiter pendant l'interview, ne lui posez pas cette question mais codez simplement "oui" à la question 101.

La question 102 sera utilisée pour évaluer le caractère approprié des pilules progestatives pour cette cliente, si c'est cette méthode qu'elle a acceptée. Pour cette question, "allaitement exclusif" veut dire seul le lait maternel et rien d'autre; pas d'eau, ni de jus ou d'autres aliments. "Presque exclusivement" veut dire le lait maternel d'abord mais parfois de l'eau ou du jus. "Complété régulièrement" veut dire que du lait maternel avec d'autres liquides et des aliments donnés aussi. Essayez de déterminer laquelle de ces catégories est plus proche du comportement actuel d'allaitement de la cliente.

\section{Quel âge avez-vous ?}

Ecrivez le nombre d'années mentionnées par la cliente. Si elle ne connaît pas son âge, essayez de l'aider à faire une estimation à l'aide d'importantes dates historiques comme repères, comme il a été indiqué au cours de la formation. Si elle n'a aucune idée de son âge, écrivez 98. 
104. Quelle est votre situation matrimoniale? Cette question devrait être répondue en utilisant la définition que la cliente a du mariage.

105. (Si pas mariée) Avez-vous un partenaire régulier?

Un partenaire régulier est quelqu'un avec lequel la cliente a des rapports sexuels régulièrement, indépendamment de la fréquence.

106. Avez-vous jamais discuté de la planification familiale avec votre mari ou votre partenaire régulier?

107. Votre mari ou votre partenaire régulier sait-il que vous utilisez ou prévoyez d'utiliser la planification familiale ? Discuter de la planification familiale entre les couples est un facteur possible affectant l'utilisation et la continuation de la planification familiale. Ces questions sont posées aussi aux clientes SMI, donc les résultats peuvent être comparés entre les clientes PF et les clientes SMI.

108. Quel est le nombre de vos propres enfants vivants?

Mettez l'accent sur les mots "vivants" et "vos propres." Cette question a pour objet de déterminer le nombre d'enfants que la femme a eus. Elle exclut les enfants adoptés et ceux des autres parents vivant présentement avec elle.

109. Quel est l'âge de votre dernier né ? Notez que l'âge du dernier né devrait être enregistré en mois.

110. Aimeriez-vous avoir (un) davantage d'enfant(s) ?

L'information obtenue de cette question peut être utilisée pour vérifier si la cliente a reçu une méthode contraceptive appropriée. Demandez "un" avec la cliente qui n'a pas d'enfant, et "davantage" avec la cliente qui a au moins un enfant.

111. (Si oui) Quand souhaitez-vous avoir (un) (le prochain) enfant?

L'information obtenue de cette question peut être utilisée pour vérifier si la cliente a reçu une méthode contraceptive appropriée.

Demandez “un” avec la cliente qui n'a pas d'enfant, et "le prochain" avec la cliente qui a au moins un enfant.

112. Pouvez-vous lire et comprendre aisément, avec difficulté ou pas du tout une lettre ou un journal ?

113. Quelles langues pouvez-vous lire et comprendre?

114. Quel est votre niveau scolaire?

115. Quelle est votre religion?

Ces quatre questions traitent du statut socioéconomique général de la cliente. Ce statut peut être utilisé pour concevoir les programmes, produire les matériels d'IEC appropriés et pour arranger les services socialement et culturellement acceptables.

Dans la question 114, notez que nous nous informons sur "fréquenté" et non pas "terminé."

116. Pour terminer, je voudrais vous poser quelques questions sur d'autres problèmes de santé de la reproduction. J'aimerais vous rappeler que l'information que vous me donnez restera strictement confidentielle et que vous n'êtes pas obligée de répondre à une question si vous n'en avez pas envie. Une femme peut tomber en état de grossesse à un moment où elle n'est pas prête pour une grossesse. Dans le passé, avez-vous jamais été en état de grossesse à un moment où vous n'étiez pas prête pour une grossesse?

117. (Si oui) Qu'aviez-vous fait la dernière fois que cela vous est arrivé ?

Ces questions sont sensibles, mais reçoivent 
généralement des réponses lorsque

l'enquêteur a développé un lien avec la

cliente, et va droit au but sans faire de jugement. Pour cette raison, ces questions

sont placées à la fin de l'interview, après que la cliente se soit habituée aux

questionnements.

118. A votre connaissance, $y$ a-t-il une maladie qui peut être transmise par les rapports sexuels?

119. A partir de ce que vous avez entendu ou lu, quels sont quelques uns des signes et symptômes courants des maladies sexuellement transmissibles?

Sachez que la cliente peut ne pas utiliser nos termes pour décrire les symptômes. Classez de votre mieux ce que la cliente dit, en utilisant nos termes. Si la cliente ne mentionne pas de problème, cochez la ligne 98.

120. Avez-vous déjà entendu parler du VIH ou du SIDA, ou bien n'êtes-vous pas sûre ?

121. A votre connaissance, quels sont les moyens par lesquels on contracte le VIH/SIDA ?

Sachez que la cliente peut ne pas utiliser nos termes pour décrire les voies de transmission. Classez de votre mieux ce que la cliente dit, en utilisant nos termes. Si la cliente ne mentionne pas de problème, cochez la ligne 98 .
122. Connaissez-vous des moyens par lesquels vous pouvez vous protéger contre les MST y compris le VHI/SIDA?

123. (Si oui) Quels sont ces moyens pour vous protéger?

Ces questions de connaissance sont extrêmement importantes. Notez que bien que le VIH/SIDA soit une maladie sexuellement transmissible, les clientes (et les prestataires) peuvent souvent la considérer comme une catégorie séparée et distincte.

124. Combien de partenaires sexuels avez-vous eu au cours des 12 derniers mois ? Ecrivez le nombre de partenaires que la cliente mentionne. Si elle dit aucun, terminez l'interview en la remerciant pour son temps et son assistance.

125. Avez-vous eu des rapports sexuels avec un nouveau partenaire au cours des 12 derniers mois?

126. (Si oui) Aviez-vous utilisé un préservatif la première fois que vous avez eu des rapports sexuels avec votre nouveau partenaire le plus récent?

Ces questions traitent du degré de risque auquel les clientes sont généralement exposées. Dans la question 126, mettez l'accent sur le fait que nous demandons la première fois que vous avez eu des rapports sexuels avec votre nouveau partenaire le plus récent. 


\section{Interview du personnel}

Cet instrument recueille les informations sur la formation, la connaissance et les pratiques des prestataires de planification familiale. Les résultats de ces interviews aident les planificateurs de programmes à concevoir des programmes de formation portant sur des besoins spécifiques du personnel. Cette information peut être comparée à celle obtenue des observations pour voir l'efficacité avec laquelle la connaissance du prestataire est traduite dans ses actes.
Les codes présentés entre accolades \{\} dans cet instrument sont des noms de variables proposés pour chaque question ou marque à cocher. On se réfère à ces noms dans le plan d'analyse (commençant à la page 166). Cependant, il faut garder à l'esprit qu'ils n'apparaîtront pas dans le questionnaire utilisé par les chercheurs sur le terrain. Voir les pages qui suivent pour l'instrument. 


\section{Interview du personnel prestataire des services de planificacion familiale / santé de la reproduction au niveau du point de prestation de services}

INSTRUCTIONS A L'ATTENTION DE L'ENQUETEUR : Tous les membres du personnel sanitaire ayant à charge la prestation des services de planification familiale, devraient être interviewés individuellement et en privé, au terme de la journée de travail. II faudra leur faire comprendre clairement que vous sollicitez leur assistance dans la recherche des voies et moyens permettant d'améliorer le fonctionnement et la qualité des services offerts par la clinique en général, et qu'il ne s'agit pas ici d'une évaluation ni de la performance de la clinique ni de leur propre performance individuelle. Pour chaque élément, veuillez encercler la réponse ou décrire comme approprié.

Structure sanitaire visitée (nom):

$$
\text { \{NOMFS\} }
$$

Code de la structure sanitaire :

$\{C O D E F S\}$

District (nom) : \{NOMDIST\}

Code du district : $\{$ CODEDIST\}

Village/ville (nom) : \{NOMVILLE\}

Code Village/ville : \{CODEVILLE\}

Numéro d'étude du prestataire : \{IDPRES\}

Date de la visite : Jour Mois Année \{DATE\}

Type de la structure sanitaire: \{TYPE $\}$

Type de secteur: \{SECT\}

$$
\begin{aligned}
& 1=\text { Hôpital de référence } \\
& 2=\text { Hôpital de district } \\
& 3=\text { Hôpital primaire } \\
& 4=\text { Centre de santé rural } \\
& 5=\text { Maternité } \\
& 6=\text { Poste de santé } \\
& 7=\text { Pharmacie } \\
& 8=\text { DBC } \\
& 9=\text { Autre }
\end{aligned}
$$

$$
\begin{aligned}
& 1=\text { Gouvernement } \\
& 2=\text { APF } \\
& 3=\text { Mission } \\
& 4=\text { Privé } \\
& 5=\text { Autre }
\end{aligned}
$$

Localité: \{LOC\}

1 = Rurale

2 = Urbaine 
Désignation du membre du personnel : \{DESIG\}

1 = Médecin

2 = Infirmière

3 = Infirmière/sage-femme

4 = Agent DBC

$5=$

Nom de l'observateur :

Signature du chef d'équipe : 


\section{Lire les salutations :}

"Bonjour (Madame/Monsieur), nous menons une étude auprès des structures sanitaires qui offrent des services de santé de la reproduction en vue d'en améliorer la qualité. Nous aimerions que vous nous parliez des expériences que vous avez eues jusqu'ici en matière de prestation de services de planification familiale. Je voudrais, avec votre autorisation, vous poser quelques questions. Rassurez-vous, cet entretien est strictement confidentiel et votre nom ne sera pas mentionné. Vous n'êtes pas aussi obligé de répondre à une question si vous n'en avez pas envie, et vous pouvez vous retirer de l'interview à n'importe quel moment. M'autorisez-vous à continuer ?"

(Si le prestataire accepte de continuer, demandez-lui s'il a des questions. Répondez correctement aux questions et ensuite poser la q.1.

Si le prestataire refuse de continuer, il faut le remercier et passer à l'interview suivante.)

\section{EXPERIENCE ET FORMATION EN SERVICES DE PF/SMI/MST}

1. J'aimerais vous poser des questions sur les services que vous dispensez aux clientes dans ce PPS. Dispensez-vous vous-même (lire de 1-18) aux clientes dans ce PPS ?

\begin{tabular}{|c|c|}
\hline 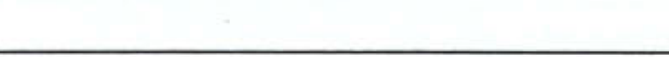 & Cochez si oul \\
\hline 1. Panification familiale & $\{S 1 \times 1\}$ \\
\hline 2. Soins prénatals & $\{S 1 \times 2\}$ \\
\hline 3. Soins de maternité/services d'accouchement & $\{\mathrm{S} 1 \times 3\}$ \\
\hline 4. Soins postnatals & $\{S 1 \times 4\}$ \\
\hline 5. Counseling IEC VIH/SIDA & $\{S 1 \times 5\}$ \\
\hline 6. Test de VIH/SIDA & $\{S 1 \times 6\}$ \\
\hline 7. Counseling/IEC sur d'autres MST & $\{S 1 \times 7\}$ \\
\hline 8. Diagnostic d'autres MST & [S1X8\}] \\
\hline 9. Traitement d'autres MST & $\{S 1 \times 9\}$ \\
\hline 10. Vaccination des enfants & $\{S 1 \times 10\}$ \\
\hline 11. Surveillance de la croissance de l'enfant & $\{S 1 \times 11\}$ \\
\hline 12. Consultation pour infécondité & $\{S 1 \times 12\}$ \\
\hline 13. Thérapie de réhydratation orale & $\{S 1 \times 13\}$ \\
\hline 14. Traitement de l'avortement incomplet & $\{\mathrm{S} 1 \mathrm{X} 14\}$ \\
\hline 15 Counseling en nutrition & $\{S 1 \times 15\}$ \\
\hline 16. Services curatifs—cliente & $\{S 1 \times 16\}$ \\
\hline 17. Services curatifs-enfant & $\{S 1 \times 17\}$ \\
\hline 18. & $\{S 1 \times 18\}$ \\
\hline
\end{tabular}


2. Depuis combien de temps travaillez-vous dans cette structure sanitaire ? \{S2\}

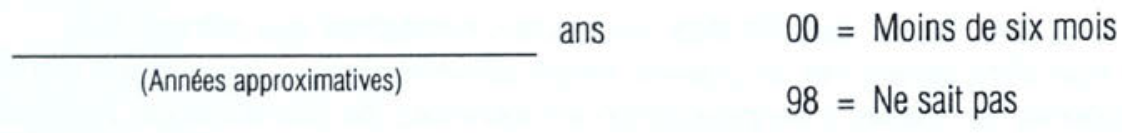

3. Depuis combien d'années avez-vous terminé votre formation de base ? \{S3\}

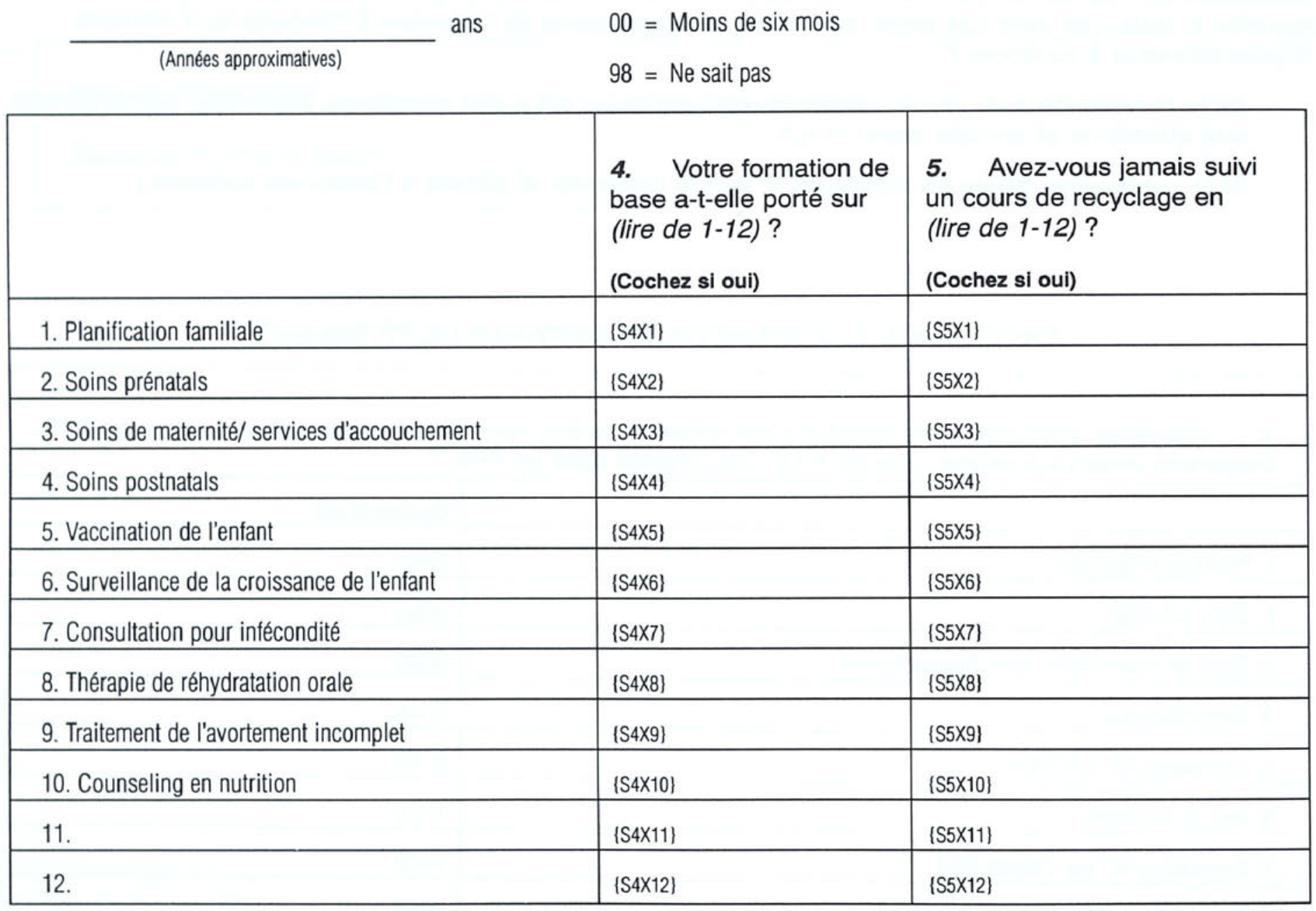


6. Après votre formation de base, avez-vous suivi un cours de recyclage ou des cours portant spécifiquement sur les compétences cliniques en planification familiale, en gestion des programmes de planification familiale ou en counseling et traitement du VIH/SIDA ? $\{$ S6\}

$1=0$ ui

2 = Non (passez à la q.9)

98 = Ne sait pas (passez à la q.9)

\begin{tabular}{|c|c|c|}
\hline & $\begin{array}{l}\text { 7. Cette structure } \\
\text { a-t-elle inclu } \\
\text { (lire de 1-19)? } \\
\text { (Cochez si oui) }\end{array}$ & $\begin{array}{l}\text { 8. (Si le thème est couvert) } \\
\text { A quand remonte cette } \\
\text { formation? } \\
0=\text { Moins d'un an } \\
98=\text { Ne sait pas }\end{array}$ \\
\hline 1. Compétences cliniques générales en PF & $\{S 7 \times 1\}$ & $\{\mathrm{S} 8 \mathrm{X} 1\}$ \\
\hline 2. Counseling en PF & [S7X2] & $\{S 8 \times 2\}$ \\
\hline 3. Pose/retrait du DIU & $\{S 7 \times 3\}$ & \{S8X3] \\
\hline 4. Pose/retrait du NORPLANT7 & $\{S 7 \times 4\}$ & $\{S 8 \times 4\}$ \\
\hline 5. ML/AL (procédure chirurgicale) & $\{S 7 \times 5\}$ & $\{\mathrm{S} 8 \times 5\}$ \\
\hline 6. Vasectomie (procédure chirurgicale) & $\{S 7 \times 6\}$ & $\{S 8 \times 6\}$ \\
\hline 7. Allaitement exclusif (MAMA) & $\{S 7 X 7\}$ & $\{S 8 \times 7\}$ \\
\hline 8. PF naturelle & $\{S 7 \times 8\}$ & $\{S 8 \times 8\}$ \\
\hline 9. Gestion & $\{S 7 X 9\}$ & $\{\mathrm{S} 8 \times 9\}$ \\
\hline 10. Supervision & $\{S 7 \times 10\}$ & $\{\mathrm{S} 8 \times 10\}$ \\
\hline 11. Gestion des statistiques & $\{S 7 \times 11\}$ & $\{\mathrm{S} 8 \times 11\}$ \\
\hline 12. Gestion du stock & $\{S 7 \times 12\}$ & $\{S 8 \times 12\}$ \\
\hline 13. Evaluation des risques/ dépistage des MST & $\{S 7 \times 13\}$ & $\{S 8 \times 13\}$ \\
\hline 14. Counseling sur les MST & $\{S 7 \times 14\}$ & $\{\mathrm{S} 8 \times 14\}$ \\
\hline 15. Diagnostic des MST en laboratoire & $\{S 7 \times 15\}$ & $\{S 8 \times 15\}$ \\
\hline $\begin{array}{l}\text { 16. Approche symptomatique du diagnostic et du } \\
\text { traitement des MST }\end{array}$ & $\{S 7 \times 16\}$ & $\{S 8 \times 16\}$ \\
\hline 17. Counseling en VIH/SIDA & $\{S 7 \times 17\}$ & $\{S 8 \times 17\}$ \\
\hline 18. Test de VIH/SIDA & $\{S 7 \times 18\}$ & [S8X18\} \\
\hline 19. Autre: & \{S7X19\} & \{S8X19\} \\
\hline
\end{tabular}


9. Avez-vous vous-même effectivement dispensé des services de PF à des clientes au cours des trois derniers mois?

$1=$ Oui

$2=$ Non (passez à la q.32)

$98=$ Ne sait pas (passez à la q.32)

10. (Si oui) Quelles méthodes avez-vous vous-même effectivement dispensées au cours des 3 derniers mois? (lire de 1 -15)

\begin{tabular}{|l|l|}
\hline & Cochez si la méthodes est fournie \\
\hline 1. Pilule combinée & $\{\mathrm{S} 10 \mathrm{X} 1\}$ \\
\hline 2. Pilule progestative & $\{\mathrm{S} 10 \mathrm{X} 2\}$ \\
\hline 3. DIU & $\{\mathrm{S} 10 \mathrm{X} 3\}$ \\
\hline 4. Injectable & $\{\mathrm{S} 10 \mathrm{X} 4\}$ \\
\hline 5. NORPLANT ${ }^{\circ}$ & $\{\mathrm{S} 10 \mathrm{X} 5\}$ \\
\hline 6. Préservatif & $\{\mathrm{S} 10 \times 6\}$ \\
\hline 7. Diaphragme & $\{\mathrm{S} 10 \mathrm{X} 7\}$ \\
\hline 8. Spermicide & $\{\mathrm{S} 10 \mathrm{X} 8\}$ \\
\hline 9. Ligature des trompes (ML/AL) & $\{\mathrm{S} 10 \mathrm{X} 9\}$ \\
\hline 10. Vasectomie & $\{\mathrm{S} 10 \mathrm{X} 10\}$ \\
\hline 11. Planification familiale naturelle & $\{\mathrm{S} 10 \mathrm{X} 11\}$ \\
\hline 12. Allaitement exclusif (MAMA) & $\{\mathrm{S} 10 \times 12\}$ \\
\hline 13. PF d'urgence & $\{\mathrm{S} 10 \times 13\}$ \\
\hline 14. Autre: & $\{\mathrm{S} 10 \times 14\}$ \\
\hline 15. & $\{\mathrm{S} 10 \times 15\}$ \\
\hline
\end{tabular}




\begin{tabular}{|c|c|c|c|}
\hline & $\begin{array}{l}\text { 11. Y a-t-il un âge minimum } \\
\text { au-dessous duquel vous ne } \\
\text { prescrirez pas vous-même } \\
\text { (lire de } 1-5 \text { ), en l'absence de } \\
\text { contre-indications médicales? }\end{array}$ & \multicolumn{2}{|c|}{$\begin{array}{l}\text { 12. (Si oui à la q.11) } \\
\text { Quel est cet âge } \\
\text { minimum? }\end{array}$} \\
\hline & (Cochez si oui) & & \\
\hline 1. Pilule (COC) & $\{S 11 \times 1\}$ & Age: & \{S12X1\} \\
\hline 2. Préservatif & $\{S 11 \times 2\}$ & Age: & $\{S 12 \times 2\}$ \\
\hline 3. DIU & $\{\mathrm{S} 11 \times 3\}$ & Age: & [S12X2\} \\
\hline 4. Injectable & $\{S 11 \times 4\}$ & Age: & $\{S 12 \times 2\}$ \\
\hline 5. Stérilisation & $\{S 11 \times 5\}$ & Age: & $\{\mathrm{S} 12 \times 2\}$ \\
\hline
\end{tabular}

\begin{tabular}{|c|c|c|}
\hline & $\begin{array}{l}\text { 13. Y a-t-il un âge maximum } \\
\text { au-delà duquel vous ne prescrirez } \\
\text { pas vous-même (lire de 1-5), en } \\
\text { l'absence de contre-indications } \\
\text { médicales? }\end{array}$ & $\begin{array}{l}\text { 14. (Si oui à la q.11) } \\
\text { Quel est cet âge } \\
\text { maximum? }\end{array}$ \\
\hline & (Cochez si oui) & \\
\hline 1. Pilule (COC) & $\{S 13 X 1\}$ & [S14X1] \\
\hline 2. Préservatif & $\{S 13 \times 2\}$ & $\{S 13 \times 2\}$ \\
\hline 3. DIU & $\{S 13 \times 3\}$ & [S13X3) \\
\hline 4. Injectable & $\{S 13 \times 4\}$ & [S13X4] \\
\hline 5. Stérilisation & $\{S 13 \times 5\}$ & $\{S 13 \times 5\}$ \\
\hline
\end{tabular}




\begin{tabular}{|c|c|c|}
\hline & $\begin{array}{l}\text { 15. Y a-t-il un nombre minimum } \\
\text { d'enfants qu'une femme doit } \\
\text { avoir avant que vous ne lui } \\
\text { prescriviez vous-même (lire de 1-5), } \\
\text { en l'absence de contre-indications } \\
\text { médicales? }\end{array}$ & $\begin{array}{l}\text { 16. (Si oul) Quel est ce } \\
\text { nombre minimum } \\
\text { d'enfants? }\end{array}$ \\
\hline & (Cochez si oui) & \\
\hline 1. Pilule (COC) & $\{S 15 \times 1\}$ & $\{\mathrm{S} 16 \mathrm{X} 1\}$ \\
\hline 2. Préservatif & [S15X2] & Nombre: \\
\hline 3. DIU & [S15X3] & Nombre: \\
\hline 4. Injectable & $\{S 15 \times 4\}$ & Nombre: \\
\hline 5. Stérilisation & $\{S 15 \times 5\}$ & Nombre: \\
\hline
\end{tabular}

\begin{tabular}{|l|l|l|}
\hline & $\begin{array}{l}\text { 17. Prescrirez-vous vous-même } \\
\text { (lire 1-5) à une femme célibataire } \\
\text { en l'absence de contre-indications } \\
\text { médicales? }\end{array}$ & $\begin{array}{l}\text { 18. Exigez-vous le } \\
\text { consentement du mari } \\
\text { avant de fournir } \\
\text { (lire de 1-5)? }\end{array}$ \\
\hline 1. Pilule (COC) & (Cochez si oui) & (Cochez si oui) \\
\hline 2. Préservatif & $\{\mathrm{S} 17 \times 1\}$ & $\{\mathrm{S} 18 \times 1\}$ \\
\hline 3. DIU & $\{\mathrm{S} 17 \times 2\}$ & $\{\mathrm{S} 18 \times 2\}$ \\
\hline 4. Injectable & $\{\mathrm{S} 17 \times 3\}$ & $\{\mathrm{S} 18 \times 3\}$ \\
\hline 5. Stérilisation & $\{\mathrm{S} 17 \mathrm{X} 4\}$ & $\{\mathrm{S} 18 \times 4\}$ \\
\hline & $\{\mathrm{S} 17 \times 5\}$ & $\{\mathrm{S} 18 \times 5\}$ \\
\hline
\end{tabular}




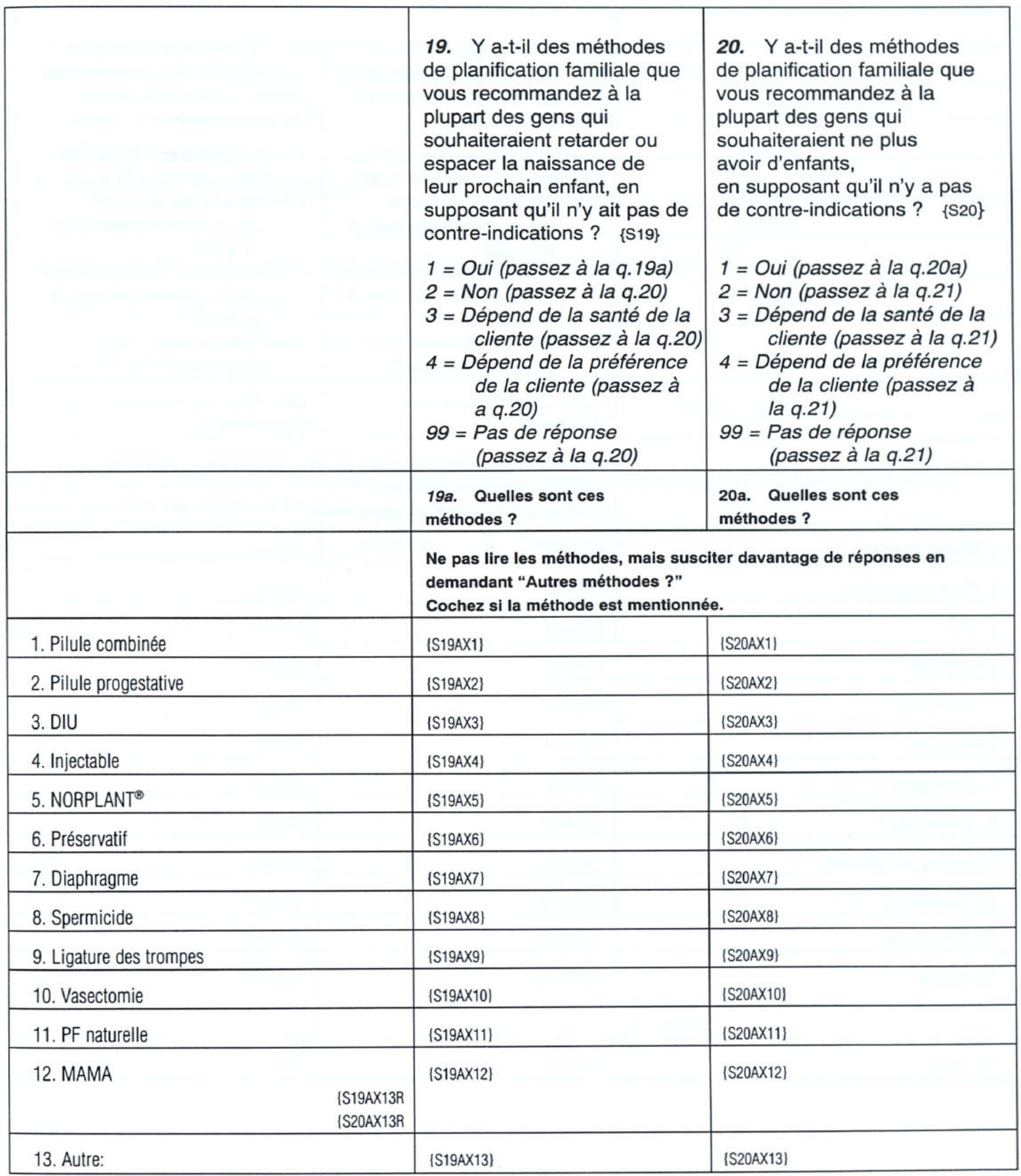




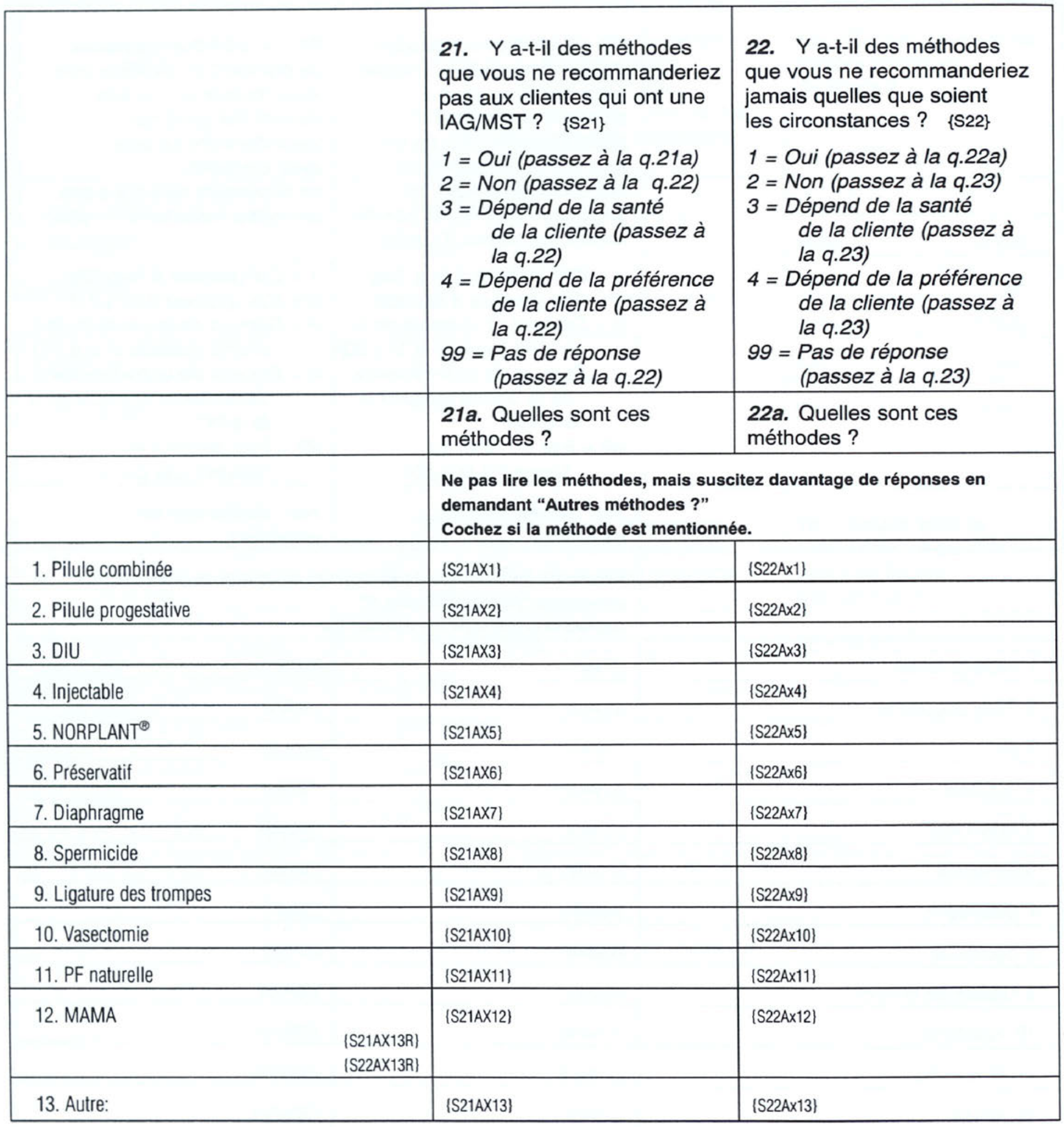


23. Que feriez-vous pour une cliente qui veut la pilule ou une autre méthode hormonale mais qui n'a pas ses règles ? (Ne pas lire mais suscitez d'autres réponses en demandant "Quoi d'autre ?")

\begin{tabular}{|l|l|}
\hline & Cochez si mentionné \\
\hline 1. Faire un test de grossesse & $\{\mathrm{S} 23 \times 1\}$ \\
\hline 2. Lui dire de revenir aux prochaines règles & $\{\mathrm{S} 23 \times 2\}$ \\
\hline $\begin{array}{l}\text { 3. Déclencher les règles } \\
\text { 4. Donner des préservatifs et lui demander de revenir dès qu'elle verra ses prochaines règles }\end{array}$ & $\{\mathrm{S} 23 \times 3\}$ \\
\hline $\begin{array}{l}\text { 5. Donner une méthode hormonale } \\
\text { 6. Donner une méthode hormonale et des préservatifs et lui demander d'utiliser les préservatifs } \\
\text { jusqu'à ses prochaines règles }\end{array}$ & \\
\hline 7. Autre: & $\{\mathrm{S} 23 \times 5\}$ \\
\hline
\end{tabular}

24. Combien de plaquettes de pilules donnez-vous d'ordinaire à une cliente qui a utilisé la pilule avec succès pendant au moins un an?

$$
\text { plaquettes } 99=\text { Autre réponse }
$$




\section{PRATIQUES EN SANTÉ DE LA REPRODUCTION}

25. Si une cliente sous pilule vient pour un contrôle/réapprovisionnement et il se trouve qu'elle court un risque élevé d'être infectée par une MST ou le VIH/SIDA, Quels conseils lui donneriez-vous ? (Ne pas lire la liste. Encerclez tout ce qui s'applique.)

$1=$ Continuer à utiliser seulement la pilule

\{S25X1\}

2 = Continuer avec la pilule mais utiliser aussi le préservatif

$\{\mathrm{S} 25 \times 2\}$

3 = Changer de la pilule au préservatif

4 = Arrêter d'utiliser tout type de contraception

\{S25X4\}

5 = Conseiller la cliente sur les MSTNIH

6 = Autre : $\{\mathrm{S} 25 \times 6 \mathrm{R}\}$

\{S25X98\}

$98=$ Ne sait pas

26. Si vous pensez qu'une cliente a une MST, que faites-vous pour elle ? (Ne pas lire la liste mais suscitez d'autres réponses en demandant, "Quoi d'autre ?")

\begin{tabular}{|c|c|}
\hline & Cochez tout ce qui est mentionné \\
\hline 1. Exiger un test de laboratoire & $\left\{\mathrm{S} 26 \mathrm{X}_{1}\right\}$ \\
\hline 2. Diagnostiquer & $\{\mathrm{S} 26 \times 2\}$ \\
\hline 3. Traiter & $\{\mathrm{S} 26 \times 3\}$ \\
\hline 4. Référer pour un diagnostic & $\{\mathrm{S} 26 \mathrm{X} 4\}$ \\
\hline 5. Référer pour un traitement & $\{\mathrm{S} 26 \times 5\}$ \\
\hline 6. Donner des conseils & $\{S 26 \times 6\}$ \\
\hline 7. Référer pour des conseils & \{S26X7\} \\
\hline 8. Donner une fiche de convocation ou d'information du partenaire & \{S26X8\} \\
\hline 9. Autre: & $\{\mathrm{S} 26 \times 9\}$ \\
\hline
\end{tabular}


27. Si vous pensez qu'une cliente a le VIH/SIDA, que faites-vous pour elle? (Ne pas lire la liste mais susciter d'autres réponses en demandant, "Quoi d'autre ?")

\begin{tabular}{|c|c|}
\hline & Cochez tout ce qui est mentionné \\
\hline 1. Demander un test de VIH & \{S27X1\} \\
\hline 2. Référer pour un test de VIH & \{S27X2\} \\
\hline 3. Donner des préservatifs & \{S27X3\} \\
\hline 4. Faire ou référer pour un traitement des complications & $\{\mathrm{S} 27 \mathrm{X} 4\}$ \\
\hline 5. Donner des conseils & $\{S 27 \times 5\}$ \\
\hline 6. Référer pour/demander de solliciter des conseils & $\{\mathrm{S} 27 \times 6\}$ \\
\hline 7. Donner une fiche de convocation ou d'information pour le partenaire & $\{S 27 \times 7\}$ \\
\hline 8. Autre: & $\{\mathrm{S} 27 \times 8\}$ \\
\hline
\end{tabular}

28. Demandez-vous un test de syphilis pour les clientes prénatales qui viennent dans cette structure sanitaire? $\{\mathrm{S} 28\}$

$$
\begin{aligned}
& 1=\text { Oui } \\
& 2=\text { Non }
\end{aligned}
$$

29. Comment vous sentez-vous en discutant de comportement sexuel relatif aux MST/NIH avec les clientes ? Etes-vous très mal à l'aise, un peu mal à l'aise, à l'aise ou très à l'aise ? \{S29\}

1 = Très mal à l'aise

2 = Un peu mal à l'aise

3 = à l'aise

4 = très à l'aise

99 = Pas de réponse

30. A votre connaissance, les femmes viennent-elles dans ce PPS pour solliciter des conseils sur l'interruption de grossesse? $\{\mathrm{S} 30\}$

$$
\begin{aligned}
& 1=\text { Oui } \\
& 2=\text { Non } \\
& 98=\text { Ne sait pas }
\end{aligned}
$$

31. A votre connaissance, les femmes viennent-elles dans cette structure sanitaire pour des traitements médicaux à cause d'un avortement incomplet ou provoqué ? \{S31\}

$$
\begin{aligned}
& 1=\text { Oui } \\
& 2=\text { Non } \\
& 98=\text { Ne sait pas }
\end{aligned}
$$




\section{CARACTÉRISTIQUES SOCIO-DÉMOGRAPHIQUES}

32. Pour terminer, je voudrais vous poser des questions sur vous-même. Quel âge avez-vous ? \{S32\} ans $\quad 98=$ Ne sait pas

33. Quelles est votre situation matrimoniale? (Insistez pour savoir la situation matrimoniale exacte) \{S33\}

$$
\begin{aligned}
& 1 \text { = Mariée/monogame } \\
& 2=\text { Mariée/polygame } \\
& 3 \text { = Cohabite/vit ensemble } \\
& 4=\text { Célibataire, jamais mariée } \\
& 5=\text { Divorcée/séparée } \\
& 6=\text { Veuve }
\end{aligned}
$$

34. Quel est le nombre de vos propres enfants vivants ? $\{\mathrm{S} 34\}$

$$
\text { enfants } \quad 99=\text { Pas de réponse }
$$

35. Quelle est votre religion ? $\{\mathrm{S} 35\}$

$$
\begin{aligned}
& 1=\text { Protestante } \\
& 2 \text { = Catholique } \\
& 3=\text { Musulmane } \\
& 4=\text { Animiste } \\
& 5=\text { Aucune } \\
& 6=\text { Autre: }
\end{aligned}
$$

$98=$ Ne sait pas

36. Sexe : $\{\mathrm{S} 36\}$

1 = Féminin

2 = Masculin 


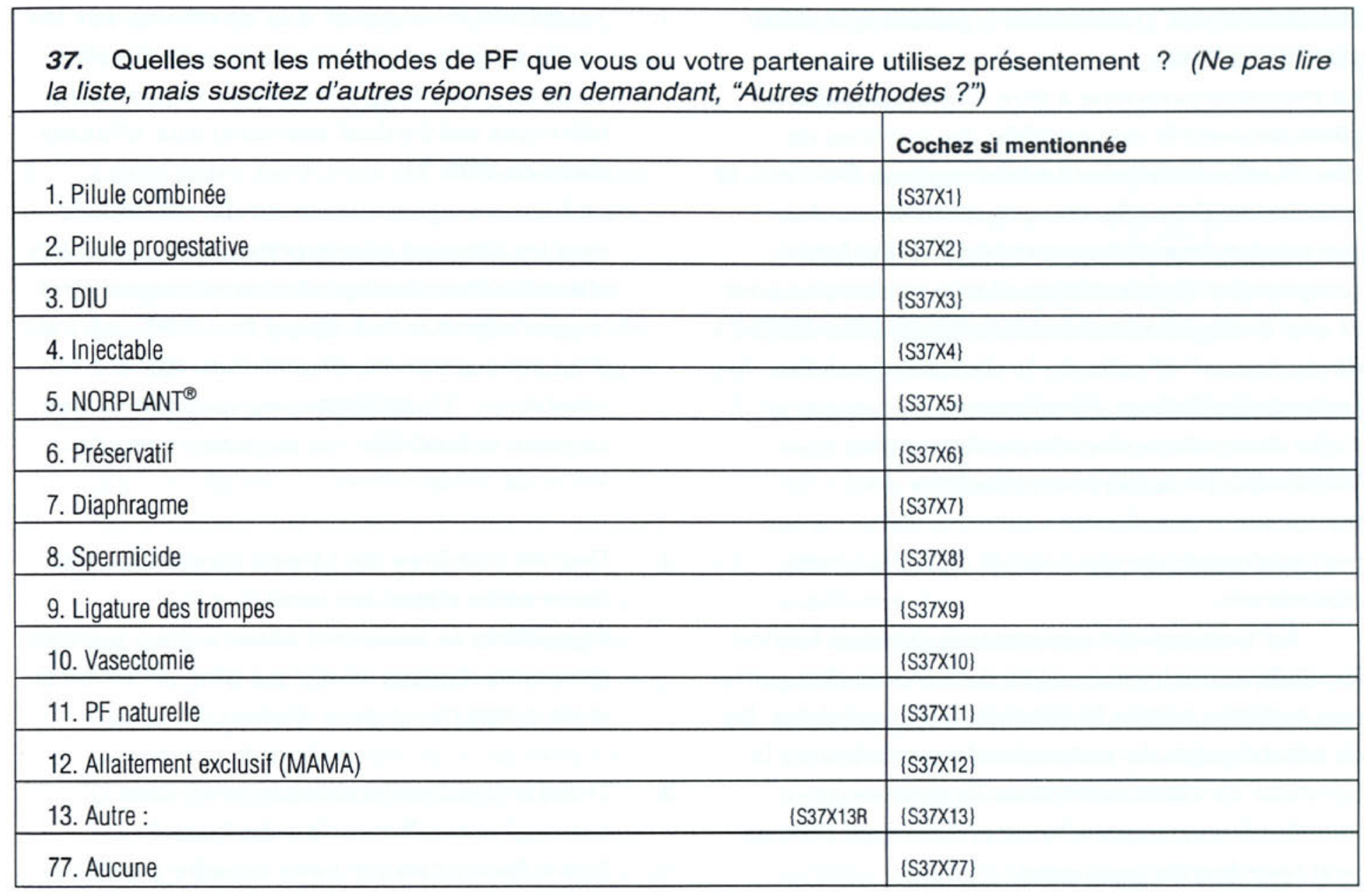

\section{Lire la conclusion :}

Merci beaucoup pour le temps que vous avez bien voulu passer avec moi. 
Interview du personnel : guide question par question

La première personne à être interviewée est généralement le responsable des services de planification familiale. Il est nécessaire d'obtenir la permission de ce dernier pour interviewer les autres membres du personnel, et de lui faire comprendre clairement que les interviews ne sont ni une évaluation de la performance individuelle du personnel, ni celle de la clinique. De même, les noms des membres du personnel ne seront pas écrits dans aucun plan d'interview. Après l'obtention de la permission auprès du responsable des services de $\mathrm{PF}$, tous les autres prestataires de services de PF devraient être interviewés.

Le responsable des services de planification familiale est celui auquel on demandera d'apporter son assistance dans la conduite de l'inventaire. De ce fait, l'équipe de recherche doit coordonner la conduite de l'inventaire avec l'interview de manière à ne pas prendre trop de temps avec un seul membre du personnel.

Les interviews devraient être réalisées à un moment où les agents ne sont pas activement engagés dans la prestation de services avec les clientes, ce qui est généralement dans l'aprèsmidi. Dans tous les cas, tous les efforts devraient être faits pour minimiser l'impact de soustraire les agents de leurs autres tâches normales. (Pour plus d'information, voir la section sur "Astuces pour réaliser une bonne interview," à la page 22.)

Avant de commencer l'interview, vous devriez remplir l'information sur la page de couverture. Si vous avez des doutes sur l'un des codes d'identification, demandez à votre Chef d'équipe. Ecrivez (ne pas signer) votre nom dans l'encadré qui se trouve au bas de la page. Lorsque vous finissez l'interview, votre Chef d'équipe la révisera et signera en bas de votre nom. Avant de commencer l'interview, rassurez-vous de lire les salutations comme elles sont imprimées dans le plan d'interview et d'obtenir le "consentement éclairé" de chaque prestataire.
1. J'aimerais vous poser des questions sur les services que vous dispensez aux clientes dans ce PPS. Dispensez-vous vous-même (diverses méthodes/ services) aux clientes dans ce PPS ?

Le but de cette question est de déterminer tous les services que le prestataire fournit aux clientes. Vous devriez vous rassurer que c'est le prestataire lui-même qui fourni les services. Pour chaque service énuméré, demandez au prestataire "Fournissez-vous vous-même (les services énumérés)," en mettant l'accent sur les mots "vous-même."

2. Depuis combien de temps travaillez-vous dans cette structure sanitaire ?

Enregistrez le nombre d'années. S'il y a moins de 6 mois, écrivez 00. S'il y a plus de six mois mais moins de 12 mois, écrivez 1 .

3. Depuis combien d'années avez-vous terminé votre formation de base ?

4. Votre formation de base a-t-elle porté sur (divers thèmes) ?

Mettez l'accent sur l'expression "formation de base." La formation de base se réfère au niveau de formation de longue durée le plus élevé que les membres du personnel ont reçu pour leur position actuelle. Par exemple, une infirmière/sage-femme peut avoir suivi la même formation qu'une infirmière, plus une formation supplémentaire de longue durée en obstétrique. Dans ce cas, "la formation de base" se réfère à la formation en obstétrique parce que c'est la formation la plus élevée qu'elle a reçue, et c'est cette formation qui la qualifie pour sa position. Pour un infirmier, "la formation de base" serait seulement la formation en infirmerie.

5. Avez-vous jamais suivi un cours de recyclage en (divers thèmes) ? Mettez l'accent sur les mots "cours de recyclage." Le cours de recyclage réfère à la formation reçue après la formation de base et 
est généralement de courte durée. Cette formation est souvent appelée formation sur le tas.

6. Après votre formation de base, avez-vous suivi un cours de recyclage ou des cours portant spécifiquement sur les compétences cliniques en planification familiale, en gestion des programmes de planification familiale ou en counseling et traitement du VIH/SIDA ?

7. Cette formation a-t-elle inclus (divers thèmes de PF) ?

8. (Si le thème est couvert) $A$ quand remonte cette formation?

Ces questions commencent à se focaliser en particulier sur la formation en planification familiale. Notez que la question 7 n'est posée que si le prestataire répond "oui" à la question 6. Pour la question 8 , ne remplissez la colonne que pour les thèmes où le prestataire a été formé. Si la formation a eu lieu il y a moins de 12 mois de cela, écrivez 0 .

9. Avez-vous vous-même effectivement dispensé des services de PF à des clientes au cours des trois derniers mois?

10. (Si oui) Quelles méthodes avez-vous vousmême effectivement dispensées au cours des 3 derniers mois?

Ces questions servent à identifier les prestataires qui fournissent en réalité des services de PF. Dans les deux questions, mettez l'accent sur l'expression "vous-même." "Effectivement dispensée" veut dire que le prestataire a physiquement donné la méthode à une cliente ou a réalisé une procédure telle que la stérilisation, la pose d'un DIU, ou une injection. Cette expression ne veut pas dire référer simplement une cliente vers un autre prestataire ou PPS pour recevoir la méthode. En ce qui concerne la MAMA et la PFN, puisque ces deux méthodes ne nécessitent pas un approvisionnement en contraceptifs ou des procédures générales, le fait de fournir l'information et l'éducation à la cliente constitue "une fourniture réelle" de la méthode.

11. Y a-t-il un âge minimum au-dessous duquel vous ne prescrirez pas vous-même (diverses méthodes), en l'absence de contre-indications médicales ?

12. (Si oui à la q.11) Quel est cet âge minimum ?

13. Y a-t-il un âge maximum au-delà duquel vous ne prescrirez pas vous-même (diverses méthodes), en l'absence de contre-indications médicales ?

14. (Si oui à la q.13) Quel est cet âge maximum ?

Ces questions permettent de savoir si le prestataire utilise l'âge comme seul critère pour fournir une méthode contraceptive à la cliente. Ces questions sont quelques-unes des plus complexes de l'Analyse Situationnelle, et vous pourrez trouver qu'elles nécessitent des explications supplémentaires au prestataire.

D'abord, demandez s'il y a un âge minimum au-dessous duquel le prestataire ne prescrira pas les méthodes. Mettez l'accent sur l'expression "vous-même." Notez qu'il n'y a pas de contre-indications médicalescette question est supposée être posée aux nouvelles clientes en bonne santé. Si le prestataire dit oui, demandez à savoir cet âge minimum et enregistrez-le. Rappelez-vous que l'âge minimum est l'âge au-dessous duquel le prestataire ne fournira pas la méthode quelles qu'en soient les circonstances. Par exemple, admettons qu'un prestataire dise qu'il ne donnera pas de pilules à une fille de 14 ans ou moins de 14 ans, à moins qu'elle ne soit mariée. Dans ce cas, le prestataire a spécifié une exception à sa règle par rapport à l'âge et n'utilise pas l'âge comme le seul critère. Dans ce cas, vous pouvez demander s'il y a un âge minimum pour les clientes mariées, qui correspondrait à 
l'exigence de l'âge minimum du prestataire, indépendamment des circonstances de la cliente. Si le prestataire ne peut pas spécifier un âge qu'il utilisera comme le seul critère, écrivez qu'il n'y a pas un âge minimum.

Le même processus s'applique à l'âge maximum. Demandez d'abord si le prestataire a un tel âge maximum, en mettant l'accent sur l'expression "vous-même" et demandez ensuite quelle est cette limite. Encore, ceci ne s'applique qu'aux nouvelles clientes PF en bon état de santé, et dans les cas où l'âge est le seul critère. Veuillez enregistrer les âges que les prestataires vous donnent, quel que soit leur caractère extrême. Par exemple, un prestataire peut dire que l'âge maximum pour le préservatif est 100. Ceci peut paraître intuitivement équivalent à "aucun âge maximum," mais il devrait être enregistré néanmoins. Ces cas seront traités dans l'analyse des données. Rappelez-vous que lorsque vous posez ces questions, et surtout si vous vous engagez dans une conversation de clarification avec le prestataire, ne faites jamais des suggestions d'échantillons d'âges. C'est-àdire, n'expliquez pas en utilisant des questions du genre "Pensez-vous qu'une cliente qui a 12 ans est trop jeune pour la pilule ?" Si vous mentionnez un nombre, même s'il ne s'agit que d'un exemple, il peut biaiser la réponse.

Parfois, des politiques de programmes spécifiques indiquent aux prestataires de ne pas donner de méthodes aux clientes en dehors de certaines limites d'âges. Par exemple, la stérilisation est souvent bannie aux femmes de moins de 35 ans, pour des considérations politiques et non pas à la discrétion du prestataire. Dans de tels cas, le prestataire peut hésiter à contredire la politique dans cette interview, même s'il le fait en cachette avec les clientes. Rassurez le prestataire que l'interview est strictement confidentielle. Cependant, s'il tient toujours à se conformer à la politique, enregistrez sa réponse.
15. Y a-t-il un nombre minimum d'enfants qu'une femme doit avoir avant que vous ne lui prescriviez vous-même (diverses méthodes), en l'absence de contreindications médicales ?

16. (Si oui) Quel est ce nombre minimum d'enfants?

Le but de la question 15 est de découvrir si le prestataire utilise un nombre minimum d'enfants comme seul critère pour fournir une méthode contraceptive à la cliente. Par exemple, le prestataire peut dire qu'il exige que la cliente ait 2 enfants avant de lui fournir l'injectable, sauf si elle est âgée de plus de 35 ans. Dans ce cas, le prestataire utilise l'âge comme un autre critère, donc le nombre d'enfants n'est pas une barrière rigide. Il faut coder cela comme aucune barrière. Notez aussi que cela implique les nouvelles clientes PF qui sont en quelque sorte bien portantes, de ce fait le prestataire n'a pas à prendre en compte les contre-indications. Mettez l'accent sur l'expression "vous-même" dans la question 15.

Parfois, les politiques de programmes spécifiques indiquent aux prestataires de ne pas donner de méthodes aux clientes qui n'ont pas d'enfants. Par exemple, les DIU sont souvent bannis chez les femmes qui n'ont pas d'enfants, pour des considérations politiques et non à la discrétion du prestataire. Dans de tels cas, le prestataire peut hésiter à contredire la politique dans cette interview, même s'il le fait en cachette avec les clientes. Rassurez le prestataire que l'interview est strictement confidentielle. Cependant, s'il persiste à se conformer à la politique, enregistrez sa réponse.

\section{Prescrirez-vous vous-même (diverses} méthodes) à une femme célibataire en l'absence de contre-indications médicales ?

18. Exigez-vous le consentement du mari avant de fournir (diverses méthodes) ? Ces deux questions traitent aussi des barrières du prestataire, mais elles sont moins 
complexes que les questions relatives à l'âge ou à la parité (11-16). Chacune d'entre elles aboutit à de simples réponses oui/non. Cependant, elles nécessitent encore que la barrière soit imposée comme seul critère. $\mathrm{Si}$ un prestataire exige généralement le consentement du mari, mais dérogera à cette exigence si la cliente a un certain nombre d'enfants vivants, alors le consentement du mari ne constitue pas le seul critère.

Souvent, des politiques de programmes spécifiques indiquent aux prestataires de ne pas donner de méthodes aux clientes qui ne sont pas mariées ou aux clientes qui n'ont pas obtenu le consentement de leur mari. Par exemple, les pilules sont souvent refusées aux clientes célibataires pour des considérations politiques et non laissé à la discrétion du prestataire. Dans de tels cas, le prestataire peut hésiter à contredire la politique dans cette interview, même s'il le fait en cachette avec les clientes. Rassurez le prestataire que l'interview est strictement confidentielle. Cependant, s'il persiste à se conformer à la politique, enregistrez sa réponse.

19. Y a-t-il des méthodes de planification familiale que vous recommandez à la plupart des gens qui souhaiteraient retarder ou espacer la naissance de leur prochain enfant, en supposant qu'il n'y ait pas de contre-indications ?

19a. Quelles sont ces méthodes?

20. Y a-t-il des méthodes de planification familiale que vous recommandez à la plupart des gens qui souhaiteraient ne plus avoir d'enfants, en supposant qu'il n'y ait pas de contre-indications ?

20a. Quelles sont ces méthodes?

21. Y a-t-il des méthodes que vous ne recommanderiez pas aux clientes qui ont une IAG/MST ?

21a. Quelles sont ces méthodes?

22. Y a-t-il des méthodes que vous ne recommanderiez jamais quels que soient les circonstances?

22a. Quelles sont ces méthodes?

Cet enchaînement de questions porte sur l'attitude du prestataire par rapport à des méthodes particulières, et les circonstances dans lesquelles il les fournirait. Dans chacun des cas, posez la question introductive (19, 20,21 et 22 ), et si la réponse est "oui", posez la question subordonnée sur les méthodes spécifiques (19a, 20a, 21a, 22a).

Les questions 19 et 20 portent sur les recommandations des méthodes d'espacement et de limitation données aux nouvelles clientes PF qui sont bien portantes et qui n'ont pas de contre-indications. La question 21 porte sur les recommandations des méthodes pour les clientes ayant des IAG/MST, en admettant que ces clientes sont par ailleurs bien portantes. C'est-à-dire qu'elles n'ont pas d'autres contre-indications. La question 22 porte sur les méthodes que le prestataire ne recommanderait jamais quel que soient les circonstances. Les réponses à cette question sont rares, puisque la plupart des prestataires reconnaissent que toutes les méthodes ont au moins des applications appropriées dans certaines circonstances. Néanmoins, quelques biais informatifs des prestataires ont été identifiés à travers cette question.

23. Que feriez-vous pour une cliente qui veut la pilule ou une autre méthode hormonale mais qui n'a pas ses règles?

Ne pas lire les réponses, mais suscitez davantage de réponses en demandant "Quoi d'autre ?" Sachez que le prestataire peut ne pas utiliser exactement le même langage mentionné dans le tableau; essayez de choisir la réponse la plus appropriée.

24. Combien de plaquettes de pilules donnezvous d'ordinaire à une cliente qui a utilisé la pilule avec succès pendant au moins un an ? Cette question vise à connaître le nombre de 
plaquettes de pilules que les prestataires comptent donner aux anciennes clientes utilisatrices de pilules. Cette information sera combinée avec celle reçue de l'observation montrant le nombre de cycles de pilules que les clientes reçoivent en réalité, et avec l'information reçue de l'inventaire montrant la situation des stocks de pilule dans ce PPS.

25. Si une cliente sous pilule vient pour un contrôle/réapprovisionnement et il se trouve qu'elle coure un risque élevé d'être infectée par une MST ou le VIH/SIDA, quels conseils lui donneriezvous?

Cette question s'informe sur le comportement du prestataire qui est difficile à observer, ex., quels conseils donnerait-il à une cliente qui coure un risque élevé d'être infectée par une MST ou le VIH/SIDA.

26. Si vous pensez qu'une cliente a une MST, que faites-vous pour elle ?

27. Si vous pensez qu'une cliente a le VIH/SIDA, que faites-vous pour elle ? Le traitement des clientes ayant des MST ou le VIH/SIDA est un sujet important pour les programmes de $\mathrm{PF}$ intégrés. Ces questions portent sur les intentions des prestataires à traiter les clientes qu'on soupçonne comme ayant ces maladies, ce qu'on peut comparer à leurs actions dans l'observation. Leurs actions peuvent être entravées par le manque de matériels de test, la gêne à discuter de MST, ou le manque de temps. Mais ces questions détermineront dans l'interview ce que les prestataires feront dans des circonstances optimales. De cette façon, toute carence dans le traitement des clientes ayant les MST/VIH/SIDA peut être identifiée comme problème lié à la formation du prestataire ou problème de mise en pratique.
28. Demandez-vous un test de syphilis pour les clientes prénatales qui viennent dans cette structure sanitaire?

Le but de cette question est de savoir si le prestataire demande normalement ou généralement un test de syphilis pour les clientes prénatales. Il s'agit là d'une mesure d'intégration des services de PF et des services de SR.

29. Comment vous sentez-vous en discutant de comportement sexuel relatif aux MST/VIH avec les clientes? Etes-vous très mal à l'aise, un peu mal à l'aise, à l'aise ou très à l'aise?

Même si un prestataire est extrêmement compétent, il peut se sentir socialement empêché de discuter de MST/VIH avec les clientes. Cette question tente de mesurer comment le prestataire se sent dans ces situations.

30. A votre connaissance, les femmes viennent-elles dans ce PPS pour solliciter des conseils sur l'interruption de grossesse ?

31. A votre connaissance, les femmes viennent-elles dans ce PPS pour des traitements médicaux à cause d'un avortement incomplet ou provoqué ? Ces questions peuvent être sujet à controverse surtout dans les pays où l'avortement est illégal. Pour cette raison, elles sont formulées attentivement. Notez que la question 30 ne demande pas si les femmes reçoivent réellement des conseils sur l'avortement ou si les femmes subissent en réalité elles-mêmes les avortements. Mais elle demande simplement si les femmes viennent solliciter de tels conseils. De même, la question 31 ne demande pas si les femmes reçoivent en réalité un traitement pour des avortements incomplets. En plus, ces deux questions évitent de demander au prestataire s'il est directement concerné par ces clientes, 
mais seulement s'il a connaissance de leur présence.

32. Pour terminer, je voudrais vous poser des questions sur vous-même. Quel âge avezvous?

Si le prestataire ne connaît pas son âge, essayez de l'aider à faire une estimation à l'aide d'importantes dates historiques comme repères comme il a été indiqué au cours de la formation. S'il n'a aucune idée de son âge, écrivez 98.

33. Quelle est votre situation matrimoniale ? Cette question devrait être répondue en utilisant la définition que la cliente a du mariage.

34. Quel est le nombre de vos propres enfants vivants?

Mettez l'accent sur les mots "vivants" et "vos propres." En ce qui concerne les femmes prestataires, cette question a pour objet de déterminer le nombre d'enfants qu'elle a eu. En ce qui concerne les hommes, cette question devrait déterminer le nombre d'enfants naturels dont il est le père. Dans les deux cas, elle exclut les enfants adoptés et ceux des autres parents vivant présentement avec eux.
35. Quelle est votre religion ?

36. Sexe?

Ces questions, avec les autres, serviront à identifier toutes différences importantes dans la religion et le sexe chez les prestataires par rapport aux clientes. De telles différences peuvent souvent constituer des barrières aux services. Notez que pour la question 36 on n'a vraiment pas besoin de la poser; marquez simplement la bonne réponse.

37. Quelles sont les méthodes de PF que vous ou votre partenaire utilisez présentement?

Ne pas lire la liste. Cochez toutes les méthodes que le prestataire déclare utiliser présentement. Parmi ceux qui utilisent des méthodes, la plupart d'entre eux citeront une seule, mais d'autres peuvent citer deux. Si le prestataire n'utilise pas de méthode, cochez 77. 


\section{Interview de sortie de la cliente SMI}

Cet instrument recueille les informations auprès des clientes SMI aussi bien sur les services qu'elles viennent juste de recevoir que sur leurs attitudes par rapport à la $\mathrm{PF}$ et à l'utilisation de la PF.

L'information recueillie auprès des clientes SMI a une double importance. D'abord, elle peut être comparée avec l'information collectée pendant l'interview de sortie de la cliente de PF. Ceci permet d'identifier les différences entre les clientes PF et les clientes SMI, et de voir si ces différences peuvent expliquer certaines attitudes et pratiques des clientes PF. Deuxièmement, les attitudes des clientes SMI par rapport à la PF et la connaissance des services de PF peuvent aider les responsables à concevoir des programmes qui attireront davantage de clientes PF. Dans cette optique, la population de clientes SMI est utilisée comme "proxy" pour réunir l'information sur la population générale qui n'utilise pas présentement la contraception.

Les codes présentés entre accolades \{\} dans cet instrument sont des noms de variables proposés pour chaque question ou marque à cocher. On se réfère à ces noms dans le plan d'analyse (commençant à la page 166). Cependant, il faut garder à l'esprit qu'ils n'apparaîtront pas dans le questionnaire utilisé par les chercheurs sur le terrain. Voir les pages qui suivent pour l'instrument. 


\section{Interview des clientes SMI fréqentant le point de prestation de services}

INSTRUCTIONS A L'ATTENTION DE L'ENQUETEUR : Ce questionnaire devrait être utilisé avec les clientes âgées de 15-45 ans dans la structure sanitaire mais qui ne sont pas venues pour des raisons de planification familiale. Si possible, essayez d'interviewer les clientes après, plutôt qu'avant, la consultation. Demandez à la femme si elle aimerait répondre à quelques questions sur les problèmes relatifs à la santé de la mère et de l'enfant. II est important d'obtenir son consentement éclairé en lisant les salutations avant de commencer l'interview.

Structure sanitaire visitée (nom) :

\{NOMFS\}

Code de la structure sanitaire :

\{CODEFS\}

District (nom) :

$\{$ NOMDIST\}

Code du district :

\{CODEDIST\}

Village/ville (nom) :

\{NOMVILLE\}

Code Village/ville :

\{CODEVILLE\}

Numéro d'étude de la cliente :

\{IDCLIENTE\}

Lieu de la cliente/salle:

Date de la visite : \{DATE\}

Jour

Mois

Année

Type de la structure sanitaire: \{TYPE\}

1 = Hôpital de référence

2 = Hôpital de district

$3=$ Hôpital primaire

$4=$ Centre de santé rural

5 = Maternité

$6=$ Poste de santé

$7=$ Pharmacie

$8=$ DBC

$9=$ Autre

Type de secteur: $\{$ SECT $\}$

1 = Gouvernement

2 = APF

$3=$ Mission

4 = Privé

$5=$ Autre

Moment de l'interview :

1 = Avant la consultation

2 = Après la consultation
Localité: $\{$ LOC $\}$

1 = Rurale

Nom de l'observateur :

Signature du chef d'équipe : 


\section{Lire les salutations :}

Bonjour Madame. Nous aimerions améliorer les services offerts par la clinique et nous pensons que votre expérience d'aujourd'hui nous serait utile. Je voudrais vous poser quelques questions sur votre visite d'aujourd'hui et vous serais très reconnaissant de me consacrer quelques minutes pour y répondre. Je ne vais pas relever votre nom, et toutes les informations que vous me donnerez resterons strictement confidentielles. Vous n'êtes pas non plus obligée de répondre à une question si vous n'en avez pas envie, et vous pouvez vous retirer de l'interview à n'importe quel moment. M'autorisez-vous à continuer ?

(Si la cliente accepte de continuer, demandez-lui si elle a des questions. Répondez correctement aux questions. Si elle a fait sa consultation, poser la q.1. Si elle n'a pas encore fait sa consultation, passez à la question 26.

Si la cliente refuse de continuer, il faut la remercier et passer à l'interview suivante.)

\section{SATISFACTION DE LA CLIENTE}

1. Quelle est la principale raison de votre visite dans ce PPS aujourd'hui ? (Encerclez une.) \{M1\}

1 = Soins prénatals

2 = Soins de maternité/ services d'accouchement

$3=$ Soins postnatals

4 = Counseling / IEC en VIH/SIDA

5 = Test de VIH/SIDA

6 = Counseling / IEC d'une autre MST

7 = Diagnostic d'une autre MST

8 = Traitement d'une autre MST

9 = Vaccination de l'enfant

10 = Surveillance de la croissance de l'enfant

11 = Consultation pour infécondité

12 = Thérapie de réhydratation orale

13 = Traitement de l'avortement incomplet

$14=$ Counseling en nutrition

15 = services curatifs - cliente

16 = Services curatifs - enfant

17 = Autre : $\{\mathrm{M} 1 \mathrm{R}\}$

2. Dans l'ensemble, êtes-vous satisfaite de votre visite d'aujourd'hui dans ce centre ou n'êtes-vous pas satisfaite de la visite?

1 = Satisfaite

2 = Non satisfaite

3 = Autre : 


\section{Quels autres services de santé avez-vous reçus ? (Lire de 1-17, en excluant la réponse de la q.1)}

\begin{tabular}{|c|c|}
\hline & Cochez si le service est reçu \\
\hline 1. Planification familiale & $\{M 3 X 1\}$ \\
\hline 2. Soins prénatals & $\{M 3 \times 2\}$ \\
\hline 3. Soins de maternité/ services d'accouchement & $\{\mathrm{M} 3 \times 3\}$ \\
\hline 4. Soins postnatals & $\{M 3 \times 4\}$ \\
\hline 5. Counseling en VIH/SIDA & $\{M 3 \times 5\}$ \\
\hline 6. Traitement en VIH/SIDA & $\{\mathrm{M} 3 \times 6\}$ \\
\hline 7. Counseling sur une autre MST & $\{M 3 \times 7\}$ \\
\hline 8. Diagnostic d'une autre MST & $\{M 3 \times 8\}$ \\
\hline 9. Traitement d'une autre MST & $\{\mathrm{M} 3 \times 9\}$ \\
\hline 10. Vaccination de l'enfant & $\{M 3 \times 10\}$ \\
\hline 11. Surveillance de la croissance de l'enfant & \{M3X11\} \\
\hline 12. Consultation pour infécondité & \{M3X12\} \\
\hline 13. Thérapie de réhydratation orale & \{M3X13\} \\
\hline 14. Traitement de l'avortement incomplet & $\{M 3 X 14\}$ \\
\hline 15. Counseling en nutrition & $\{M 3 X 15\}$ \\
\hline 16. Services curatifs - cliente & $\{M 3 \times 16\}$ \\
\hline 17. Services curatifs - enfant & $\{M 3 \times 17\}$ \\
\hline 77. Pas d'autres services & \{M3X77\} \\
\hline
\end{tabular}

4. Avez-vous le sentiment qu'aujourd'hui vous avez reçu l'information et les services que vous vouliez? $\{\mathrm{M} 4\}$

$$
\begin{aligned}
& 1=\text { Oui } \\
& 2=\text { Non } \\
& 3=\text { Partiellement } \\
& 98=\text { Ne sait pas }
\end{aligned}
$$

5. Avez-vous le sentiment que votre consultation avec l'agent clinique a été trop courte, trop longue ou juste le temps nécessaire? $\{\mathrm{M} 5\}$

$$
\begin{aligned}
& 1 \text { = Trop courte } \\
& 2 \text { = Trop longue } \\
& 3=\text { Juste le temps nécessaire } \\
& 98=\text { Ne sait pas }
\end{aligned}
$$


6. Pendant cette visite, aviez-vous des préoccupations par rapport aux problèmes de santé que vous vouliez discuter avec le prestataire? $\{\mathrm{M} 6\}$

$$
\begin{aligned}
& 1 \text { = Oui } \\
& 2=\text { Non (passez à la q.8) } \\
& 98=\text { Ne sait pas (passez à la q.8) }
\end{aligned}
$$

7. (Si oui) Le prestataire a-t-il écouté vos préoccupations de manière satisfaisante ? \{M7\}

$$
\begin{aligned}
& 1=\text { Oui } \\
& 2=\text { Non } \\
& 98=\text { Ne sait pas }
\end{aligned}
$$

8. Pendant cette visite, aviez-vous des questions que vous vouliez poser? $\{\mathrm{M} 8\}$

$$
1=\text { Oui }
$$

2 = Non (passez à la q.11)

98 = Ne sait pas (passez à la q.11)

9. (Si oui) Le prestataire vous a-t-il donné le temps de les poser ? $\{\mathrm{M} 9\}$

$$
1=\text { Oui }
$$

2 = Non (passez à la q.11)

98 = Ne sait pas (passez à la q.11)

10. (Si oui) Le prestataire a-t-il répondu à vos questions jusqu’à ce que vous ayez satisfaction? \{M10\}

$1=$ Oui

$2=$ Non

$98=$ Ne sait pas

11. Au cours de cette visite, le prestataire a-t-il fait des examens ou procédures de santé ? $\{$ M11\}

$$
1=\text { Oui }
$$

2 = Non (passez à la q.14)

98 = Ne sait pas (passez à la q.14)

12. (Si oui) Le prestataire a-t-il expliqué les examens ou procédures avant de les faire ? $\{\mathrm{M} 12\}$

1 = Oui

$2=$ Non

$98=$ Ne sait pas

13. Le prestataire a-t-il expliqué les résultats des examens ou procédures ? $\{$ M13\}

$$
\begin{aligned}
& 1=\text { Oui } \\
& 2=\text { Non } \\
& 98=\text { Ne sait pas }
\end{aligned}
$$


14. A votre avis, y avait-il assez d'intimité pendant votre consultation avec le prestataire de services ?

1 = Oui

$2=$ Non

$98=$ Ne sait pas

15. Au cours de la consultation, avez-vous eu le sentiment que le prestataire était facile à comprendre lorsqu'il vous expliquait certaines choses, ou bien avez-vous le sentiment qu'il était difficile à comprendre ? $\{$ M15\}

1 = Facile à comprendre

2 = Difficile à comprendre

$98=$ Ne sait pas

16. Environ combien de temps vous a-t-il fallu attendre entre le moment où vous êtes arrivée dans ce centre et celui auquel vous avez commencé à recevoir les services pour lesquels vous êtes venue ? \{M16\}

$$
\text { minutes } \quad 98=\text { Ne sait pas }
$$

(S'il n'y a pas de temps d'attente, passez à la q.18)

17. Pensez-vous que votre temps d'attente était raisonnable ou trop long ? $\{\mathrm{M} 17\}$

1 = Raisonnable

2 = Trop long

$98=$ Ne sait pas

18. Si une de vos amies désirait les services pour lesquels vous êtes venue ici aujourd'hui, l'encourageriezvous à venir dans ce PPS ou l'encourageriez-vous à aller ailleurs ? \{M18\}

1 = Venir dans ce PPS

2 = Aller ailleurs

$98=$ Ne sait pas

19. Qu'est-ce que vous avez aimé le plus de votre visite d'aujourd'hui ? \{M19\}

20. Qu'est-ce que vous avez détesté le plus de votre visite d'aujourd'hui ? $\{$ M20\}

21. Si vous pouviez suggérer une amélioration aux services fournis, quelle serait cette suggestion? \{M21\} 


\section{ACTIVITES D'IEC}

22. Pendant cette visite, vous a-t-on donné ou avez-vous pris un dépliant ou un matériel éducatif pour l'amener à la maison? \{M22\}

$1=$ Oui

2 = Non (passez à la q.24)

$98=$ Ne sait pas (passez à la q.24)

23. (Si oui) Quel était le(s) thème(s) de ce matériel ? (Ne pas lire la liste, mais suscitez d'autres réponses en demandant, "D'autres thèmes ?")

\begin{tabular}{|c|c|}
\hline & Cochez si le thème est mentionné \\
\hline 1. Planification familiale & $\{M 23 X 1\}$ \\
\hline 2. Soins prénatals/postnatals & $\{\mathrm{M} 23 \times 2\}$ \\
\hline 3. Services d'accouchement & $\{\mathrm{M} 23 \times 3\}$ \\
\hline 4. VIH/SIDA & $\{$ IM23X4\} \\
\hline 5. Autres MST & $\{\mathrm{M} 23 \times 5\}$ \\
\hline 6. Bien-être de l'enfant & $\{$ M23X6\} \\
\hline 7. Nutrition & \{M23X7\} \\
\hline 8. Autre: & \{M23X8\} \\
\hline 98. Ne sait pas & \{M23X98\} \\
\hline
\end{tabular}

24. Avez-vous pris part à une causerie de groupe dans cette structure sanitaire aujourd'hui ? \{M24\}

$1=$ Oui

2 = Non (passez à la q.26)

98 = Ne sait pas (passez à la q.26) 
25. (Si oui) Quels sont les thèmes qui ont été couverts par la causerie de groupe ? (Ne pas lire la liste, mais suscitez d'autres réponses en demandant, "D'autres thèmes ?")

\begin{tabular}{|c|c|}
\hline & Cochez si le thème est mentionné \\
\hline 1. Planification familiale & $\{M 25 \times 1\}$ \\
\hline 2. Soins prénatals & $\{\mathrm{M} 25 \times 2\}$ \\
\hline 3. Soins maternels/ services d'accouchement & $\{\mathrm{M} 25 \times 3\}$ \\
\hline 4. Soins postnatals & $\{$ (M25X4\} \\
\hline 5. VIH/SIDA & \{M25X5\} \\
\hline 6. MST & $\{$ M25X6\} \\
\hline 7. Vaccination de l'enfant & $\{$ \{25X7\} \\
\hline 8. Surveillance de la croissance de l'enfant & $\{$ [M25X8\} \\
\hline 9. Infécondité & $\{$ M25X9\} \\
\hline 10. Thérapie de réhydratation orale & $\{M 25 \times 10\}$ \\
\hline 11. Traitement de l'avortement incomplet & $\{M 25 \times 11\}$ \\
\hline 12. Counseling en nutrition & $\{\mathrm{M} 25 \times 12\}$ \\
\hline 13. Services curatifs-clientes & $\{\mathrm{M} 25 \times 13\}$ \\
\hline 14. Services curatifs - enfant & $\{\mathrm{M} 25 \times 14\}$ \\
\hline 15. Allaitement maternel & $\{\mathrm{M} 25 \times 15\}$ \\
\hline 16. Autre: & $\{\mathrm{M} 25 \times 16\}$ \\
\hline 98. Ne sait pas & $\{M 25 \times 98\}$ \\
\hline
\end{tabular}




\section{ACCESSIBILITÉ}

26. Les heures d'ouverture de ce PPS vous conviennent-elles ? \{M26\}

1 = Oui (passez à la q.28)

$2=$ Non

$98=$ Ne sait pas (passez à la q.28)

27. (Si non) Quelle heure serait la plus convenable pour vous ? (Encerclez une.) \{M27\}

1 = Tôt le matin

2 = Pendant l'heure du repas

3 = Après-midi

4 = Soir $/$ nuit

5 = Week-ends / jours fériés

6 = Autre :

$98=$ Ne sait pas

28. Avez-vous jamais été renvoyée de ce PPS pendant les horaires officiels de travail ? $\{$ M28\}

1 = 0ui

$2=$ Non

3 = Aucune expérience précédente avec le PPS

$98=$ Ne sait pas

29. Combien de temps vous a-t-il fallu pour venir ici aujourd'hui ? \{M29\}

minutes $\quad 98=$ Ne sait pas

30. Quel a été le principal moyen de transport que vous avez utilisé pour venir ici ? (Encerclez une seule réponse.) $\{\mathrm{M} 30\}$

$1=$ Marche

2 = Charrette

$3=$ Bus $/$ combi

4 = Bicyclette

5 = Voiture personnelle

$6=$ Autre : 
31. A votre connaissance, quels sont les services généralement fournis dans ce PPS ? (Ne pas lire, mais suscitez d'autres réponses en demandant "Autres services ?")

\begin{tabular}{|c|c|}
\hline & Cochez si le service est mentionné \\
\hline 1. Planification familiale & $\{M 31 \times 1\}$ \\
\hline 2. Soins prénatals & $\{M 31 \times 2\}$ \\
\hline 3. Soins de maternité/ services d'accouchement & $\{M 31 \times 3\}$ \\
\hline 4. Soins postnatals & $\{M 31 \times 4\}$ \\
\hline 5. Counseling/IEC en VIH/SIDA & $\{M 31 \times 5\}$ \\
\hline 6. Test de VIH/SIDA & $\{M 31 \times 6\}$ \\
\hline 7. Counseling/IEC d'une autre MST & $\{M 31 \times 7\}$ \\
\hline 8. Diagnostic d'une autre MST & $\{M 31 \times 8\}$ \\
\hline 9. Traitement d'une autre MST & $\{M 31 \times 9\}$ \\
\hline 10. Vaccination de l'enfant & $\{M 31 \times 10\}$ \\
\hline 11. Surveillance de la croissance de l'enfant & $\{\mathrm{M} 31 \times 11\}$ \\
\hline 12. Consultation pour infécondité & $\{\mathrm{M} 31 \times 12\}$ \\
\hline 13. Thérapie de réhydratation orale & $\{\mathrm{M} 31 \times 13\}$ \\
\hline 14. Traitement de l'avortement incomplet & $\{\mathrm{M} 31 \times 14\}$ \\
\hline 15. Counseling en nutrition & \{M31X15\} \\
\hline 16. Services curatifs-cliente & $\{\mathrm{M} 31 \times 61\}$ \\
\hline 17. Services curatifs — enfant & $\{\mathrm{M} 31 \times 17\}$ \\
\hline 18. = Autre : & [M31X18] \\
\hline 98. Ne sait pas & [M31X98) \\
\hline
\end{tabular}

32. A part ce PPS, y a-t-il un autre endroit près de chez vous où vous pouvez aller pour les mêmes services pour lesquels vous êtes venue ici aujourd'hui ? $\{\mathrm{M} 32\}$

$$
\begin{aligned}
& 1=\text { Oui } \\
& 2=\text { Non (passez à la q.35) } \\
& 98=\text { Ne sait pas (passez à la q.35) }
\end{aligned}
$$


33. (Si oui) Quel type de PPS est-ce? (Encerclez une seule réponse. S'il y a plus d'un PPS, choisissez le plus proche de chez elle.)

$$
\begin{aligned}
& 1=\text { DBC } \\
& 2 \text { = Poste de santé } \\
& 3=\text { Centre de santé } \\
& 4=\text { Hôpital } \\
& 5=\text { Autre: }
\end{aligned}
$$

$98=$ Ne sait pas

34. Quelle est la raison principale pour laquelle vous n'êtes pas allée là-bas pour ces services ? \{M34\} (Ne pas lire la liste. Insistez pour connaître la principale raison et encerclez une seule réponse.)

$1=$ Horaires d'ouverture non convenables

2 = Prend trop de temps pour aller là-bas

3 = Piètre qualité des services

4 = Peu de services disponibles

5 = Veut garder l'anonymat

6 = A d'autres raisons de venir ici (ex. services de vaccination)

7 = Plus cher là-bas

\begin{tabular}{|c|c|c|}
\hline & $\begin{array}{l}\text { 35. Au cours des } 12 \\
\text { derniers mois, combien de } \\
\text { visites avez-vous faites } \\
\text { dans ce centre/hôpital pour } \\
\text { (lire de 1-4)? }\end{array}$ & $\begin{array}{l}\text { 36. Au cours des } 12 \\
\text { derniers mois, combien de } \\
\text { visites avez-vous faites } \\
\text { dans un autre centre/hôpital } \\
\text { pour (lire } 1 \text {-4)? }\end{array}$ \\
\hline 1. Planification familiale & $\{M 35 \times 1\}$ & $\{M 36 \times 1\}$ \\
\hline 2. Bien-être de l'enfant & $\{M 35 \times 2\}$ & $\{\mathrm{M} 36 \times 2\}$ \\
\hline 3. Soins prénatals & $\{$ IM35X3\} & $\{\mathrm{M} 36 \times 3\}$ \\
\hline 4. $\mathrm{VIH} / \mathrm{SIDA}$ & \{M35X4\} & $\{M 36 \times 4\}$ \\
\hline
\end{tabular}

8 = Préfère le prestataire d'ici

9 = Autre : \{M34R\}

$98=$ Ne sait pas 
37. Maintenant je voudrais vous poser des questions sur le coût de votre transport ainsi que les services que vous avez reçus dans cette clinique. Combien avez-vous payé pour (lire 1-6) ?

\begin{tabular}{|c|c|}
\hline & $\begin{array}{l}\text { Enregistrez le montant } \\
\text { (en monnaie locale) } \\
98=\text { Ne sait pas }\end{array}$ \\
\hline 1. Consultation & \{M37X1\} \\
\hline 2. Médicaments & \{M37X1] \\
\hline $\begin{array}{l}\text { 3. Autres éléments tels que le coton, les gants, les produits antiseptiques } \\
\text { ou les aiguilles }\end{array}$ & $\{M 37 \times 1\}$ \\
\hline 4. Carte d'inscription/de membre & \{M37X1\} \\
\hline 5. Voyage & \{M37X1\} \\
\hline 6. Autre: & $\{M 37 X 1\}$ \\
\hline
\end{tabular}

38. Dans l'ensemble, pensez-vous que le coût d'obtention des services est beaucoup trop cher, un peu trop cher ou acceptable pour vous?

1 = Beaucoup trop cher

2 = Un peu trop cher

3 = Acceptable

$98=$ Ne sait pas 
39. A présent je voudrais vous poser des questions sur la maternité. Connaissez-vous des moyens ou méthodes que les femmes et les hommes peuvent utiliser pour retarder ou empêcher la grossesse ? \{M39\}

$$
\begin{aligned}
& 1=\text { Oui } \\
& 2=\text { Non (passez à la q.49) }
\end{aligned}
$$

\begin{tabular}{|c|c|c|}
\hline & $\begin{array}{l}\text { 40. (Si oui) Veuillez me } \\
\text { citer ces méthodes que vous } \\
\text { connaissez. (Ne pas lire mais } \\
\text { suscitez d'autres réponses } \\
\text { en demandant, "Autres } \\
\text { méthodes?") }\end{array}$ & $\begin{array}{l}\text { 41. (Si mentionnée) A votre } \\
\text { connaissance, est-il possible } \\
\text { d'obtenir cette méthode } \\
\text { dans ce PPS? }\end{array}$ \\
\hline & $\begin{array}{l}\text { Cochez si la méthode est } \\
\text { mentionnée.) }\end{array}$ & Cochez si oui \\
\hline 1. Pilule combinée (ou non spécifiée) & $\{M 40 X 1\}$ & $\{M 41 X 1\}$ \\
\hline 2. Pilule progestative & $\{\mathrm{M} 40 \times 2\}$ & $\{M 41 \times 2\}$ \\
\hline 3. DIU & $\{\mathrm{M} 40 \times 3\}$ & $\{\mathrm{M} 41 \times 3\}$ \\
\hline 4. Injectable & \{M40X4\} & $\{$ M41X4\} \\
\hline 5. NORPLANT ${ }^{\circledR}$ & $\{M 40 \times 5\}$ & $\{$ M41X5\} \\
\hline 6. Préservatif & $\{M 40 X 6\}$ & $\{\mathrm{M} 41 \times 6\}$ \\
\hline 7. Diaphragme & $\{M 40 \times 7\}$ & $\{M 41 \times 7\}$ \\
\hline 8. Spermicide & $\{M 40 X 8\}$ & $\{M 41 \times 8\}$ \\
\hline 9. Ligature des trompes & $\{$ M40X9\} & $\{M 41 X 9\}$ \\
\hline 10. Vasectomie & $\{M 40 \times 10\}$ & $\{M 41 \times 10\}$ \\
\hline 11. PF naturelle & $\{M 40 \times 11\}$ & $\{M 41 \times 11\}$ \\
\hline 12. MAMA & $\{\mathrm{M} 40 \times 12\}$ & $\{M 41 \times 12\}$ \\
\hline 13. Autre: & $\{M 40 \times 13\}$ & $\{M 41 \times 13\}$ \\
\hline
\end{tabular}

42. Avez-vous entendu parler ou vu quelque chose relative à la planification familiale aujourd'hui dans cette structure sanitaire? $\{\mathrm{M} 42\}$

$$
\begin{aligned}
& 1 \text { = Oui } \\
& 2=\text { Non (passez à la q.44) } \\
& 98=\text { Ne sait pas (passez à la q.44) }
\end{aligned}
$$


43. (Si oui) Avez-vous (lire 1-5)?

\begin{tabular}{|l|l|}
\hline & Cochez si oui \\
\hline 1. Vu une affiche sur la planification familiale? & $\{$ M43X1\} \\
\hline $\begin{array}{l}\text { 2. Vu une brochure, une boîte à images ou une fiche d'information sur la } \\
\text { planification familiale? }\end{array}$ & $\{\mathrm{M43 \times 2 \}}$ \\
\hline 3. Entendu parler de PF pendant une causerie sanitaire? & $\{$ M43X3\} \\
\hline 4. Entendu parler de PF pendant une consultation? & $\{\mathrm{M} 43 \times 4\}$ \\
\hline 5. Vu des échantillons de contraceptifs? & $\{\mathrm{M} 43 \times 5\}$ \\
\hline
\end{tabular}

44. Approuvez-vous ou désapprouvez vous les couples qui utilisent une méthode de planification familiale pour éviter la grossesse? \{M44\}

1 = Approuve

2 = Désapprouve

$98=$ Ne sait pas / pas d'opinion

45. Utilisez-vous ou votre époux/partenaire utilise-t-il présentement une méthode pour espacer, éviter ou prévenir une grossesse? $\{\mathrm{M} 45\}$

1 = Oui

2 = Non (passez à la q.47)

$98=$ Ne sait pas (passez à la q.47)

46. (Si oui) Quelle méthode? (Encerclez une.) $\{\mathrm{M} 46\}$

1. Pilule combinée (ou non spécifiée)

2. Pilule progestative

3. DIU

4. Injectable

5. NORPLANT ${ }^{\circledR}$

6. Préservatif

7. Diaphragme

8. Spermicide

9. Ligature des trompes

10. Vasectomie

11. PFN (passez à la q.49)

12. MAMA (passez à la q.49)

13. $=$ Autre : [M46R] 
47. Où irez-vous/êtes-vous allez pour avoir votre méthode de PF actuelle ? $\{$ M47\}

1 = Dans cette structure sanitaire

2 = Une autre structure sanitaire

3 = Pharmacie/ boutique/ pharmacien/ drugstore

$4=\mathrm{DBC} / \mathrm{EBF}$

5 = Médecin privé

$6=$ Autre :

\{M47R\}

48. Quelle est la principale raison pour laquelle vous obtenez votre méthode dans cet endroit et non pas ailleurs? $\{\mathrm{M} 48\}$

(Ne pas lire, mais insistez pour savoir la principale raison. Encerclez une.)

1 = Ne connaît que cette seule source

$2=$ Horaires d'ouverture convenables

$3=$ Qualité des services

4 = Gamme de services à offrir

5 = Peut garder l'anonymat

$6=$ Proche de la maison

7 = Coat des méthodes

$8=$ Aime/fait confiance au prestataire

9 = Autre :

[M48R]

$98=$ Ne sait pas 


\section{CARACTERISTIQUES SOCIO-DÉMOGRAPHIQUES}

49. Maintenant, je voudrais vous poser quelques questions sur vous-même. Allaitez-vous actuellement? $\{\mathrm{M} 49\}$

$$
\begin{aligned}
& 1=\text { Oui } \\
& 2=\text { Non (passez à la q.51) }
\end{aligned}
$$

50. (Si oui) Allaitez-vous exclusivement, presque exclusivement, ou complétez-vous régulièrement avec des aliments et/ou des jus? $\{$ M50\}

$$
\begin{aligned}
& 1 \text { = Allaite exclusivement } \\
& 2 \text { = Presque exclusivement } \\
& 3 \text { = Complété régulièrement avec des aliments et/ou des jus } \\
& 98 \text { = L'interviewer est incapable de déterminer le degré de suppléments d'aliments }
\end{aligned}
$$

51. Quel âge avez-vous? \{M51\}

$$
\text { ans } \quad 98=\text { Ne sait pas }
$$

52. Quelle est votre situation matrimoniale ? (Insistez pour savoir la situation matrimoniale exacte.) [M52]

1 = Mariée / monogame (passez à la q.54)

2 = Mariée / polygame (passez à la q.54)

3 = Cohabite (passez à la q.54)

4 = célibataire, jamais mariée

5 = Divorcée / séparée / veuve

53. (Si pas mariée) Avez-vous un partenaire régulier? \{M53\}

$1=$ Oui

2 = Non (passez à la q.56)

54. Avez-vous jamais discuté de la planification familiale avec votre mari ou votre partenaire régulier? $\{\mathrm{M} 54\}$

$1=$ Oui

$2=$ Non

98 = Ne sait pas

55. Pensez-vous que votre mari/partenaire approuve ou désapprouve que vous utilisiez une méthode pour espacer ou éviter une grossesse ? $\{M 55\}$

1 = Approuve

2 = Désapprouve

$98=$ Ne sait pas 
56. Quel est le nombre de vos propres enfants vivants ? $\{$ M56 enfants

(Si aucun, passez à la q.58)

57. Quel est l'âge de votre dernier né ? $\{M 57\}$

$$
\text { mois } 98=\text { Ne sait pas }
$$

58. Aimeriez-vous avoir davantage d'enfants? (Encerclez une.) \{M58\}

$1=$ Oui

2 = Non (passez à la q.60)

3 = Dépend du mari (passez à la q.60)

4 = Dépend de Dieu (passez à la q.60)

5 = N'est pas sâre / indécise (passez à la q.60)

59. (Si oui) Quand souhaitez-vous avoir (un) (le prochain) enfant ? (Encerclez une.) \{M59\}

$1=$ Dans moins d'un an

$2=$ Dans un an

$3=$ Entre un et deux ans

$4=$ Deux ans

5 = Plus de deux ans

6 = Toute autre réponse

$98=$ Ne sait pas

60. Pouvez-vous lire et comprendre aisément, avec difficulté ou pas du tout une lettre ou un journal ? \{M60\}

1 = Aisément

2 = Avec difficulté

$3=$ Pas du tout

61. Quelles langues pouvez-vous lire et comprendre? (Ne pas lire la liste, mais suscitez d'autres réponses en demandant, "Autres langues ?")

\begin{tabular}{|c|c|c|}
\hline & & Cochez si mentionnée \\
\hline 1. Anglais/Français & & $\{M 61 X 1\}$ \\
\hline 2. Dialecte local & & $\{$ \{M61X2\} \\
\hline 3. Autre: & \{M61X3R\}) & $\{M 61 \times 3\}$ \\
\hline 4. Autre: & \{M61X4R\} & $\{M 61 \times 4\}$ \\
\hline 5. Autre: & \{M61X5R\} & $\{M 61 \times 5\}$ \\
\hline
\end{tabular}


62. Quel est votre niveau scolaire? (Encerclez une.) \{M62\}

$$
\begin{aligned}
& 1 \text { = N'a pas été à l'école } \\
& 2 \text { = Primaire } \\
& 3=\text { Fondamentale } \\
& 4=\text { Secondaire } \\
& 5=\text { Supérieur } \\
& 6=\text { Post-universitaire }
\end{aligned}
$$

63. Quelle est votre religion? (Encerclez une.) $\{\mathrm{M} 63\}$

$$
\begin{aligned}
& 1 \text { = Protestante } \\
& 2 \text { = Catholique } \\
& 3=\text { Musulmane } \\
& 4=\text { Animiste } \\
& 5=\text { Aucune } \\
& 6=\text { Autre: }
\end{aligned}
$$

\section{PROBLEMES RELATIFS A LA SANTE DE LA REPRODUCTION}

64. Pour terminer, je voudrais vous poser quelques questions sur d'autres problèmes de santé de la reproduction. J'aimerais vous rappeler que l'information que vous me donnez restera strictement confidentielle et que vous n'êtes pas obligée de répondre à une question si vous n'en avez pas envie. II peut arriver qu'une femme tombe en état de grossesse à un moment où elle n'est pas prête pour une grossesse. Dans le passé, avez-vous jamais été en état de grossesse à un moment où vous n'étiez pas prête pour une grossesse? \{M64\}

$$
\begin{aligned}
& 1 \text { = Oui } \\
& 2=\text { Non (passez à la q.66) } \\
& 98=\text { Ne sait pas / pas de réponse (passez à la q.66) }
\end{aligned}
$$

65. (Si oui) Qu'aviez-vous fait la dernière fois que cela vous est arrivé ? (Encerclez une.) \{M65\}

1 = Rien, a porté la grossesse à terme

2 = A essayé de mettre fin à la grossesse mais sans succès, a accouché

3 = A essayé et réussi à mettre fin à la grossesse

4 = Autre: $\{\mathrm{M} 65 \mathrm{R}\}$

98 = Ne sait pas / pas de réponse 
66. A votre connaissance, y a-t-il une maladie qui peut être transmise par les rapports sexuels ? $\{$ M66\}

$1=$ Oui

2 = Non (passez à la q.69)

98 = Ne sait pas (passez à la q.69)

67. (Si oui) Avez-vous entendu ou lu sur les signes et symptômes courants des maladies sexuellement transmissibles? (M67)

$1=$ Oui

2 = Non (passez à la q.69)

$98=$ Ne sait pas (passez à la q.69)

68. (Si oui) Quels sont quelques-uns des signes et symptômes courants des maladies sexuellement transmissibles? (Ne pas lire la liste, mais suscitez d'autres réponses en demandant, "Autres signes ou symptômes ?')

\begin{tabular}{|c|c|}
\hline & Cochez si mentionné \\
\hline 1. Pertes vaginales anormales & $\{M 68 \times 1\}$ \\
\hline 2. Saignement vaginal anormal & $\{$ M68X2\} \\
\hline 3. Démangeaison de l'appareil génital & $\{\mathrm{M} 68 \times 3\}$ \\
\hline 4. Lésions / plaies & $\{$ M68X4\} \\
\hline 5. Douleur au bas ventre & $\{M 68 \times 5\}$ \\
\hline 6. Douleur pendant les rapports sexuels & $\{$ M68X6\} \\
\hline 7. Douleur pendant l'urine & $\{$ M68X7\} \\
\hline 8. Croissance anormale au niveau de l'appareil génital (verrues) & $\{$ M68X8\} \\
\hline 9. Ecoulement urétral & $\{$ M68X9\} \\
\hline 10. Perte de poids & $\{M 68 \times 10\}$ \\
\hline 11. Diarrhée de longue durée & $\{M 68 \times 11\}$ \\
\hline 12. Autre: & $\{M 68 \times 12\}$ \\
\hline
\end{tabular}

69. Avez-vous jamais entendu parler du VIH ou du SIDA, ou bien n'êtes-vous pas sûre ? \{M69\}

$1=$ Oui

2 = Non (passez à la q.72)

$98=$ Ne sait pas (passez à la q.72)

70. Avez-vous jamais entendu parler des moyens par lesquels on contracte le VIH/SIDA ? $\{$ M70\}

1 = Oui

2 = Non (passez à la q.72)

$98=$ Ne sait pas (passez à la q.72) 
71. A votre connaissance, quels sont les moyens par lesquels on contracte le VIH/SIDA ?

(Ne pas lire la liste mais suscitez d'autres réponses en demandant, "Autres moyens ?")

\begin{tabular}{|c|c|}
\hline & Cochez si mentionné \\
\hline 1. Rapports sexuels & $\{M 71 \times 1\}$ \\
\hline 2. Transfusion sanguine & $\{M 71 \times 2\}$ \\
\hline 3. Le fait de partager les objets tels que lames de rasoir ou aiguilles & $\{M 71 \times 3\}$ \\
\hline 4. De la mère à l'enfant & $\{M 71 \times 4\}$ \\
\hline$\{M 71 \times 5 R\}$ & $\{M 71 \times 5\}$ \\
\hline
\end{tabular}

72. Connaissez-vous des moyens par lesquels vous pouvez vous protéger contre les MST y compris le $\mathrm{VIH} / \mathrm{SIDA}$ ? $\{\mathrm{F} 122\}$

1 = Oui

2 = Non (passez à la q.74)

$98=$ Ne sait pas (passez à la q.74)

73. (Si oui) Quels sont ces moyens pour vous protéger? (Ne pas lire la liste mais suscitez d'autres réponses en demandant, "Autres moyens ?")

\begin{tabular}{|c|c|}
\hline & Cochez si mentionné \\
\hline 1. Rester fidèle à votre seul époux/partenaire & $\{M 73 \times 1\}$ \\
\hline 2. Encourager l'époux/partenaire à rester fidèle & $\{\mathrm{M} 73 \times 2\}$ \\
\hline 3. Utiliser les préservatifs & $\{\mathrm{M} 73 \times 3\}$ \\
\hline 4. Eviter de partager les aiguilles, rasoirs, etc. & $\{M 73 \times 4\}$ \\
\hline 5. Autre: & $\{M 73 \times 5\}$ \\
\hline
\end{tabular}

74. Combien de partenaires sexuels avez-vous eus au cours des 12 derniers mois ? $\{$ M74\} partenaires

(Si aucun, passez à la conclusion)

75. Avez-vous eu des rapports sexuels avec un nouveau partenaire au cours des 12 derniers mois? \{M75\}

$1=$ Oui

$2=$ Non (passez à la conclusion)

98 = Ne sait pas (passez à la conclusion) 
76. (Si oui) Si oui, aviez-vous utilisé un préservatif la première fois que vous avez eu des rapports sexuels avec votre nouveau partenaire le plus récent ?

$$
\begin{aligned}
& 1=\text { Oui } \\
& 2=\text { Non } \\
& 98=\text { Ne sait pas }
\end{aligned}
$$

\section{Lire la conclusion :}

Merci beaucoup pour le temps que vous avez bien voulu consacrer à répondre à nos questions. 
Interview de sortie de la cliente SMI : guide question par question

La cliente vient juste de recevoir les services de SMI. Soyez sensible au fait que la cliente peut être venue de loin, qu'elle a attendu longtemps les services, et peut avoir subi une rude épreuve. Cependant, elle peut accepter une interview avec une personne sensible et intéressante. (Pour plus d'informations, voir la section "Astuces pour réaliser une bonne interview" à la page 22.)

Il faut conduire la cliente dans un endroit privé pré-choisi, loin du personnel clinique et des autres clientes et ensuite lire les salutations. Ces salutations sont extrêmement importantes parce qu'elles rassurent que la cliente a donné son "consentement éclairé" pour participer à l'étude. Demandez à la cliente si elle a des questions à poser sur l'étude. Si nécessaire, vous pouvez encourager un peu la cliente en lui disant, "Votre participation sera d'une très grande aide," mais dans tous les cas vous devez respecter la volonté de la cliente si elle ne veut pas être interviewée. $\mathrm{Si}$ elle refuse de participer, il faut la remercier et passer à la cliente suivante.

Vous devriez remplir les informations des deux premières pages avant de commencer l'interview. Si vous avez des doutes sur l'un des codes, demandez à votre Chef d'équipe. Ecrivez (ne pas signer) votre nom dans l'encadré en bas de la page. Lorsque vous finirez l'interview, votre Chef d'équipe la révisera et signera en bas de votre nom. En ce qui concerne le reste de l'interview, suivez les instructions pour enregistrer les réponses de la cliente en encerclant une réponse pré-codée, en cochant un encadré ou en écrivant une réponse comme demandé.

Certaines questions sont des questions filtres, c'est-à-dire qu'elles font une distinction de certaines clientes par rapport à d'autres pour poser davantage de questions. Par exemple, la question 26 repartit les clientes entre celles qui trouvent que les horaires du centre sont convenables et celles qui pensent le contraire. Les clientes qui pensent que les horaires ne sont pas convenables répondront à la question de savoir quelles seront les horaires les plus convenables. Ces questions filtres incluent toujours des "exemples de saut" qui sautent les questions qui ne sont pas applicables à certaines clientes. Faites très attention à tous les exemples de saut. A la fin de l'interview, révisez vite vos réponses codées pour voir si elles sont au complet et correctes, et rassurez-vous que vous avez suivi les sauts correctement. Si possible, corrigez toutes les erreurs alors que la cliente est encore disponible.

1. Quelle est la principale raison de votre visite dans ce PPS aujourd'hui ? Demandez à la cliente la principale raison pour laquelle elle est venue aujourd'hui dans ce centre. Elle aura l'occasion d'énumérer plus tard tous les services qu'elle a reçus (question 3).

2. Dans l'ensemble, êtes-vous satisfaite de votre visite aujourd'hui dans ce PPS ou n'êtes-vous pas satisfaite?

Souvent les clientes hésitent à exprimer tout mécontentement général parce qu'elles veulent être courtoises. Cependant elles ont tendance à exprimer plus leur mécontentement avec des questions plus spécifiques. Les questions 4 à 21 de la présente interview donneront à la cliente plus d'opportunité de critiquer spécifiquement les services.

3. Quels autres services de santé avez-vous reçus aujourd'hui ?

Lire chacun des services en excluant la réponse de la cliente à la question 1 comme étant la principale raison de la visite. Cochez (marque de vérification) la colonne correspondant à chaque service reçu. Si la cliente n'a reçu aucun service, cochez 77 .

4. Avez-vous le sentiment qu'aujourd'hui vous avez reçu l'information et les services que vous vouliez?

Les clientes se rendent dans un PPS pour obtenir des informations et des services 
particuliers. L'objet de cette question est de voir si la cliente a reçu l'information et les services qu'elle désirait.

\section{Pensez-vous que votre consultation} PF avec l'agent de santé a été trop courte, trop longue, ou juste le temps qu'il faut ?

Cette information peut être comparée avec la satisfaction des clientes par rapport à la durée de leur consultation.

6. Pendant cette visite, aviez-vous des inquiétudes par rapport aux problèmes de santé que vous vouliez discuter avec le prestataire?

7. (Si oui) Le prestataire a-t-il écouté vos préoccupations jusqu'à ce que vous ayez satisfaction?

Le terme "inquiétudes" est très vaste. Les inquiétudes incluent mais ne se limitent pas aux soucis ou aux rumeurs des risques de santé, à la santé de l'enfant, au fait de s'exposer aux MST/VIH/SIDA, comment garder l'intimité, etc. Si la cliente dit qu'en réalité elle avait des inquiétudes, posez-lui la question 7 .

8. Pendant cette visite, aviez-vous des questions que vous vouliez poser?

9. (Si oui) Le prestataire vous a-t-il donné le temps de les poser?

10. (Si oui) Etes-vous satisfaite des réponses que le prestataire a données à vos questions?

Le fait que la cliente soit libre de poser des questions et qu'elle soit satisfaite des réponses données est un bon indicateur de la qualité des relations interpersonnelles. Ces trois questions cherchent à savoir si la cliente se sentait à l'aise en demandant l'information dont elle avait besoin.

"Les questions" peuvent porter sur n'importe quel sujet tel que le mode de transmission d'une infection, ou pour obtenir des conseils nutritionnels, etc. Les questions peuvent aussi porter sur des "inquiétudes" comme définies ci-dessus.

La cliente peut tenir compte de plusieurs aspects en répondant à la question 10 : par exemple, le caractère complet, l'exactitude ou la manière que le prestataire a utilisé dans ses réponses. N'assistez pas la cliente à répondre à cette question parce qu'elle est la seule qui puisse déterminer sa propre satisfaction avec les réponses à ses questions.

11. Pendant cette visite, le prestataire a-t-il fait des examens ou des procédures de santé ?

12. (Si oui) Le prestataire a-t-il expliqué les examens ou procédures avant de les faire?

13. Le prestataire a-t-il expliqué les résultats des examens ou procédures de santé ? "Les examens de santé" incluent les examens de la tête jusqu'aux pieds, les examens mammaires, les examens pelviens, et l'examen de toute autre partie du corps. "Les procédures" incluent la prise de la tension artérielle ou de la température, la pose d'un DIU, une injection, etc.

L'idée qui sous-tend ces trois questions est que si les clientes connaissent les examens et les procédures, elles seront plus à l'aise pendant leur visite. Une explication de l'examen ou de la procédure implique le fait de dire à la cliente ce que le prestataire s'apprête à faire, pourquoi il le fait, et ce que la cliente peut sentir. L'explication des résultats de l'examen ou de la procédure implique le fait de dire à la cliente si l'infirmière/sage-femme est arrivée à une conclusion ou un diagnostic à partir de l'examen. Si un diagnostic n'est pas disponible immédiatement, alors l'explication des résultats inclurait le fait de dire à la cliente qu'elle sera contactée plus tard pour les résultats. 
14. A votre avis, y avait-il assez d'intimité pendant votre consultation avec le prestataire de services ?

Il s'agit là de l'avis de la cliente, de ce fait, c'est elle qui le détermine.

15. Au cours de la consultation, avez-vous eu le sentiment que le prestataire était facile à comprendre lorsqu'il vous expliquait certaines choses, ou bien était-il difficile à comprendre?

Il s'agit là de l'avis de la cliente, de ce fait, c'est elle qui le détermine.

16. Environ combien de temps vous a-t-il fallu attendre entre le moment où vous êtes arrivée à ce PPS et celui auquel vous avez commencé à recevoir les services pour lesquels vous êtes venue?

Le but de cette question est de déterminer le temps d'attente de la cliente. Encouragez la cliente à faire une estimation du temps pendant lequel elle a attendu si elle n'a pas de montre et/ou si elle n'a pas relevé le temps. Réservez la mention "ne sait pas" pour les clientes qui ne sont pas en mesure de faire une estimation même si on leur demande de le faire.

17. Avez-vous le sentiment que votre temps d'attente était raisonnable ou trop long ? Il s'agit là de l'opinion de la cliente sur l'ensemble du temps d'attente qu'elle a fait avant de commencer à recevoir les services pour lesquels elle était venue. Il ne faut pas considérer le début des procédures d'inscription comme étant les services pour lesquels elle est venue.

18. Si une de vos amies désirait les services pour lesquels vous êtes venue ici aujourd'hui, l'encourageriez-vous à venir dans ce PPS ou l'encourageriez-vous à aller ailleurs?

Parfois, les clientes choisissent un centre de santé sur recommandations des amies. Cette question permet de savoir si les clientes SMI recommandent ce PPS à des amies, ce qui constitue une mesure "proxy" de la satisfaction de la cliente.

19. Qu'est-ce que vous avez aimé le plus de votre visite d'aujourd'hui ?

Mettez l'accent sur les mots "le plus."

Certaines clientes peuvent détester tout, dans ce cas vous devriez passer à la question 20.

20. Qu'est-ce que vous avez détesté le plus de votre visite d'aujourd'hui ?

Mettez l'accent sur les mots "le plus."

Certaines clientes peuvent ne pas avoir quelque chose à redire, ou peuvent ne pas se sentir à l'aise pour raconter ce qui leur à déplu. Dans ces cas, laissez la question sans réponse.

21. Si vous aviez une amélioration à suggérer par rapport aux services fournis, laquelle alliez-vous suggérer?

Cette importante question permet à la cliente de mentionner quelque chose qui l'a gênée dans cette expérience, sans être trop grave. Incitez la cliente avec des commentaires de soutien du genre: "Tout ce que vous voulez suggérer est bon."

22. Au cours de cette visite, vous a-t-on donné ou avez-vous pris un dépliant ou un matériel éducatif pour l'amener à la maison?

23. (Si oui) Quel est le thème de ce matériel ? Aucune distinction n'est faite entre dépliants, brochures, prospectus ou tout autre matériel éducatif.

Essayez de trouver le thème du dépliant ou du matériel éducatif donné à la cliente. Ne lisez pas la liste, mais suscitez davantage de réponses en demandant "Y a-t-il d'autres thèmes?" Nous sommes intéressés par la 
connaissance que la cliente a du matériel qu'elle a reçu. Si elle aimerait se référer à ses matériels et vous dire les thèmes, il faut marquer les réponses. Cependant, si elle vous montre simplement les matériels, ne regardez pas vous-même pour marquer leurs thèmes, mais demandez "Sur quoi portent ces objets ?"

\section{Avez-vous pris part à une causerie de} groupe dans cette structure sanitaire aujourd'hui?

La "causerie de groupe" est souvent appelée "causerie sanitaire." Elle est généralement dirigée par une infirmière/sage-femme, et peut inclure plusieurs thèmes. Elle est fréquemment sous la forme d'un cours magistral, mais lorsqu'elle est bien faite, elle peut inclure des questions, des expositions d'échantillons ou autres supports audiovisuels. "Prendre part" et "entendre" sont synonymes dans cette question.

25. (Si oui) Quels sont les thèmes qui ont été abordés pendant cette causerie de groupe ?

Ne posez cette question que lorsque la cliente a pris part à une causerie de groupe. Nous sommes intéressés par l'information dont la cliente se rappelle pendant la causerie sanitaire, donc, il ne faut pas lui souffler en lisant la liste des thèmes. Il faut remarquer aussi le sondage approprié, "Autres thèmes?"

26. Les heures d'ouverture de ce PPS sontelles convenables pour vous?

27. (Si non) Quel est le temps qui vous serait le plus convenable?

Les heures d'ouverture des services peuvent être une grande barrière pour les clientes, et ces questions portent sur la convenance des horaires de la clinique. Même dans les contextes où les horaires de la clinique sont fixés par le ministère de la Santé ou par une autorité centrale, les informations issues de ces questions peuvent être très utiles pour obtenir des changements de ces politiques si nécessaire.

28. Avez-vous jamais été renvoyée de ce PPS pendant les heures officielles de travail ? "Renvoyée" veut dire qu'on a refusé de lui fournir les services ou bien on lui a dit de revenir une autre fois. Remarquez qu'il s'agit là de l'antécédent de la cliente avec le PPS et non pas de la visite d'aujourd'hui. Ceci implique aussi le renvoi à cause du fait qu'il y a trop de clientes ou autre raison non spécifiée. Si la cliente est venue une seule fois, a rencontré un prestataire qui lui a dit de revenir pour une procédure à une date ultérieure, elle n'est pas comptée comme une "renvoyée" pour cette question.

29. Combien de temps vous a-t-il fallu pour arriver ici aujourd'hui ?

Nous nous informons sur le temps de voyage de la cliente depuis son domicile parce que le temps de voyage peut constituer parfois une grande barrière aux services. Si la cliente est venue de son lieu de travail ou de chez un parent, il faut calculer le temps à partir de làbas. Encouragez la cliente à estimer le temps approximatif en minutes.

30. Quel a été le principal moyen de transport que vous avez utilisé pour venir ici ?

La cliente peut avoir utilisé deux ou trois, voire plusieurs moyens de transport-la marche et le minibus seraient une combinaison courante. Lorsque la cliente utilise deux ou plusieurs moyens de transport, il faut l'encourager à décider lequel est le principal moyen. Tout critère qu'elle utilise pour décider-temps, distance, coût, etc.-est bon.

31. A votre connaissance, quels types de services autres que la planification familiale sont généralement fournis dans ce PPS ? Cette question traite de la connaissance d'autres services par la cliente, ce qui sera 
comparée plus tard avec les services réellement disponibles. Cela permet de savoir si certains services sont sous utilisés simplement à cause du fait que les gens ne les connaissent pas.

Sachez que la cliente n'utilisera pas nos termes exacts. Faites de votre mieux pour interprétez ses commentaires selon les catégories. Si la cliente dit "MST" ou "VIH," vous pouvez la sonder pour savoir en posant la question "Quels types de services de MST ou de VIH sont généralement fournis ?" afin de vous rapprocher plus près des catégories de codes disponibles.

32. En dehors de ce PPS, y a-t-il un autre endroit près de chez vous où vous pouvez obtenir des services de planification familiale?

33. (Si oui) Quel est le type de cette structure sanitaire ?

34. Quelle serait la principale raison pour laquelle vous n'êtes pas allée solliciter des services là-bas?

Le choix d'une cliente pour des PPS est important parce qu'il peut être motivé par la qualité des services qu'elle a reçus. Ces trois questions portent sur les clientes qui ont choisi de venir dans ce centre au lieu d'un autre.

Si la cliente dit qu'il y a un autre PPS près de son domicile ou de son lieu de travail, ou une autre place qu'elle visite régulièrement, il faut coder la question 32 en conséquence. Ensuite les questions 33 et 34 devraient être posées, et la cliente encouragée à penser à un seul PPS, celui qui est le plus proche de sa maison ou de son lieu de travail. Dans la question 34 , la cliente peut mentionner plus d'une raison. Encouragez-la à choisir la principale raison.

35. Au cours des $\mathbf{1 2}$ derniers mois, combien de visites avez-vous faites dans ce centre/hôpital pour (divers services) ?
36. Au cours des 12 derniers mois, combien de visites avez-vous faites dans un autre centre/hôpital pour (divers services) ? Ces questions mesurent le niveau d'utilisation des services chez les clientes SMI, à la fois dans ce PPS et ailleurs.

37. A présent, j'aimerais vous poser des questions sur le coût de votre transport et les services que vous avez reçus dans cette clinique. Combien avez-vous payé pour (divers services et voyage) ?

Les clientes peuvent connaître le total payé sans savoir comment les charges ont été reparties. Si c'est le cas, faites une note à la marge avec le montant total.

38. Dans l'ensemble, pensez-vous que le coût total pour obtenir les services est beaucoup trop cher, un peu trop cher ou acceptable pour vous ?

Puisque le fardeau des dépenses ne peut être jugé que par rapport au revenu de la cliente, cette question donne à la cliente l'occasion d'évaluer les difficultés qu'elle éprouve pour payer les services, même si le coût est assez bas.

39. A présent je voudrais vous poser des questions sur la maternité. Connaissezvous des moyens ou méthodes que les femmes et les hommes peuvent utiliser pour retarder ou empêcher la grossesse?

40. (Si oui) Veuillez me dire ces méthodes que vous connaissez.

Ces questions mesurent la connaissance des clientes SMI par rapport à la planification familiale. Si la cliente a entendu parler de certaines méthodes, posez la question 40 mais ne lisez pas la liste des méthodes. Laissez la cliente vous dire toutes les méthodes qu'elle peut mentionner spontanément. Suscitez davantage de réponses en demandant "Autres méthodes?" Sachez que la cliente peut ne pas utiliser les 
termes employés dans la liste des méthodes.

En utilisant nos termes, classez de votre mieux ce que la cliente dit.

41. (Si mentionnée) A votre connaissance, est-il possible d'obtenir cette méthode dans ce PPS ?

Pour chaque méthode que la cliente mentionne, demandez-lui si cette méthode peut être obtenue dans ce PPS. Cette information sera vérifiée par rapport à la disponibilité de cette méthode dans ce PPS, ce qui donnera aux responsables une idée sur la connaissance que les clientes ont de leurs services.

42. Avez-vous entendu parler ou vu quelque chose relative à la planification familiale aujourd'hui dans cette structure sanitaire ?

43. (Si oui) Avez-vous (vu une affiche/ une brochure/assisté à une causerie sanitaire/etc.)?

Bien que la majeure partie de l'Analyse Situationnelle porte sur la manière dont les services de SR sont intégrés aux services de $\mathrm{PF}$, ces deux questions cherchent à savoir si la PF est intégrée aux services de SMI.

44. Approuvez-vous ou désapprouvez-vous (des couples qui utilisent une méthode de planification familiale pour éviter la grossesse?

Cette question qui traite de l'attitude est une importante question indirecte de l'acceptabilité de la PF au sein de la population générale. Probablement, toutes les clientes PF approuvent l'utilisation de la $\mathrm{PF}$, donc leurs attitudes par rapport à la PF ne sont pas représentatives de la population générale. Les clientes SMI, bien que n'étant pas forcément représentatives de la population générale, peuvent donner une meilleure idée sur les attitudes générales. Les résultats de cette question peuvent aussi informer les responsables des programmes sur la réceptivité des messages de PF par la population des clientes SMI.

45. Utilisez-vous ou votre époux/partenaire utilise-t-il présentement une méthode pour espacer, éviter ou prévenir une grossesse?

46. (Si oui) Quelle méthode ?

Ces résultats peuvent être comparés à ceux de la PF pour le type de méthode utilisée. Les méthodes utilisées par le mari/ partenaire(s) telles que les préservatifs, la vasectomie et le retrait sont considérées aussi comme étant celles de la cliente.

47. Où irez-vous/êtes-vous allée pour avoir votre méthode de PF actuelle?

48. Quelle est la principale raison pour laquelle vous obtenez votre méthode dans cet endroit et non pas ailleurs?

Ces questions portant sur l'approvisionnement en contraceptifs donnent une importante information sur les points utilisés par les femmes qui pourraient ne plus retourner dans ce PPS pour des services de PF. Par exemple, si leurs réponses à la question 48 montrent qu'elles pensent que la qualité des services est meilleure ailleurs, alors le responsable du programme aura identifié un important problème.

49. Maintenant, je voudrais vous poser quelques questions sur vous-même. Allaitez-vous actuellement?

50. (Si oui) Allaitez-vous exclusivement, presque exclusivement, ou complétezvous régulièrement avec des aliments et/ou des jus ?

Si la cliente est en train d'allaiter pendant l'interview, ne lui posez pas cette question mais codez simplement "oui" à la question 49 .

Quant à la question 50, "allaitement exclusif" veut dire seul le lait maternel et rien d'autre; pas d'eau, ni de jus ou d'autres 
aliments. "Presque exclusivement" veut dire le lait maternel d'abord mais parfois de l'eau ou du jus. "Complété régulièrement" veut dire que du lait maternel avec d'autres liquides et des aliments donnés aussi. Essayez de déterminer laquelle de ces catégories est plus proche du comportement actuel d'allaitement de la cliente.

\section{Quel âge avez-vous ?}

Ecrivez le nombre d'années mentionnées par la cliente. Si elle ne connaît pas son âge exact, essayez de l'aider à faire une estimation à l'aide d'importantes dates historiques comme repères, comme il a été indiqué au cours de la formation. Si elle n'a aucune idée de son âge, écrivez 98.

52. Quelle est votre situation matrimoniale ? Cette question devrait être répondue en utilisant la définition que la cliente a du mariage.

\section{3. (Si pas mariée) Avez-vous un partenaire} régulier?

Un partenaire régulier est quelqu'un avec lequel la cliente a des rapports sexuels régulièrement, indépendamment de la fréquence.

54. Avez-vous jamais discuté de la planification familiale avec votre mari ou votre partenaire régulier?

55. Votre mari ou votre partenaire régulier sait-il que vous utilisez ou prévoyez d'utiliser la planification familiale ? Discuter de la planification familiale entre les couples est un facteur possible affectant l'utilisation et la continuation de la planification familiale. Ces questions portent sur le nombre de discussions et d'approbations relatives à la PF qui se passent entre les couples qui n'utiliseraient pas la PF pour le moment. Ce résultat peut être comparé à des résultats similaires chez les clientes PF.
56. Quel est le nombre de vos propres enfants vivants?

Mettez l'accent sur les mots "vivants" et "vos propres." Cette question a pour objet de déterminer le nombre d'enfants que la femme a eus. Elle exclut les enfants adoptés et ceux des autres parents vivant présentement avec elle.

57. Quel est l'âge de votre dernier né ? Notez que l'âge du dernier né devrait être enregistré en mois.

58. Aimeriez-vous avoir davantage d'enfants ? Demandez "un" à la cliente qui n'a pas d'enfant, et "davantage" à la cliente qui a au moins un enfant.

59. (Si oui) Quand souhaitez-vous avoir (un) (le prochain) enfant ?

Demandez "un" avec la cliente qui n'a pas d'enfant, et "le prochain" avec la cliente qui a au moins un enfant.

60. Pouvez-vous lire et comprendre aisément, avec difficulté ou pas du tout une lettre ou un journal ?

61. Quelles langues pouvez-vous lire et comprendre?

62. Quel est votre niveau scolaire ?

63. Quelle est votre religion?

Ces quatre questions traitent du statut socioéconomique général de la cliente. Ce statut peut être utilisé pour concevoir les programmes, produire les matériels d'IEC appropriés et pour arranger les services socialement et culturellement acceptables.

Dans la question 62, notez que nous nous informons sur "fréquenté" et non pas "terminé."

64. Pour terminer, je voudrais vous poser quelques questions sur d'autres problèmes de santé de la reproduction. J'aimerais vous rappeler que l'information 
que vous me donnez restera strictement confidentielle et que vous n'êtes pas obligée de répondre à une question si vous n'en avez pas envie. Une femme peut tomber en état de grossesse à un moment où elle n'est pas prête pour une grossesse. Dans le passé, avez-vous jamais été en état de grossesse à un moment où vous n'étiez pas prête pour une grossesse?

65. (Si oui) Qu'aviez-vous fait la dernière fois que cela vous est arrivé ?

Ces questions sont sensibles, mais reçoivent généralement des réponses lorsque l'enquêteur a développé un lien avec la cliente, et va droit au but sans faire de jugement. Pour cette raison, ces questions sont placées à la fin de l'interview, après que la cliente se soit habituée aux questionnements.

66. A votre connaissance, y a-t-il une maladie qui peut être transmise par les rapports sexuels?

67. (Si oui) Avez-vous entendu ou lu sur les signes et symptômes courants des maladies sexuellement transmissibles?

68. Quels sont quelques-uns des signes et symptômes courants des maladies sexuellement transmissibles ?

Sachez que la cliente peut ne pas utiliser nos termes pour décrire les symptômes. Classez de votre mieux ce que la cliente dit, en utilisant nos termes.

69. Avez-vous déjà entendu parler du VIH ou du SIDA, ou bien n'êtes-vous pas sûre?

70. Avez-vous jamais entendu parler des moyens par lesquels on contracte le VIH/SIDA ?
71. A votre connaissance, quels sont les moyens par lesquels on contracte le VIH/SIDA ?

Sachez que la cliente peut ne pas utiliser nos termes pour décrire les voies de transmission. Classez de votre mieux ce que la cliente dit, en utilisant nos termes.

72. Connaissez-vous des moyens par lesquels vous pouvez vous protéger contre les MST y compris le VIH/SIDA?

73. (Si oui) Quels sont ces moyens pour vous protéger?

Ces questions de connaissance sont extrêmement importantes. Notez que bien que le VIH/SIDA soit une maladie sexuellement transmissible, les clientes (et les prestataires) peuvent souvent la considérer comme une catégorie séparée et distincte.

74. Combien de partenaires sexuels avez-vous eus au cours des 12 derniers mois ?

Ecrivez le nombre de partenaires que la cliente mentionne. Si elle dit aucun, terminez l'interview en la remerciant pour son temps et son assistance.

75. Avez-vous eu des rapports sexuels avec un nouveau partenaire au cours des 12 derniers mois?

76. (Si oui) Si oui, aviez-vous utilisé un préservatif la première fois que vous avez eu des rapports sexuels avec votre nouveau partenaire le plus récent? Ces questions traitent du degré de risque auquel les clientes sont généralement exposées. Dans la question 76, mettez l'accent sur le fait que nous demandons la première fois que vous avez eu des rapports sexuels avec votre nouveau partenaire le plus récent. 


\section{Analyse des données et rédaction des rapports}

\section{Saisie et nettoyage des données}

Une fois la collecte des données terminée, les données doivent être saisies sur ordinateur en vue de leur analyse. Les questionnaires remplis devraient être centralisés et rangés selon la date de visite, le district ou tout autre critère. Après le rangement des questionnaires, leurs numéros devraient être écrits en haut et dans le coin droit de chaque instrument. Ces numéros devraient commencer par 1 pour chaque instrument, et seront utilisés pour identifier chaque questionnaire. Ce processus est utile à la phase de nettoyage et d'analyse des données.

En fonction de la taille de l'AS, la saisie des données peut nécessiter deux à six semaines. Parfois, les chercheurs engagent des membres de l'équipe de collecte des données pour faire la saisie des données, ce qui exige une certaine maîtrise de l'ordinateur. La saisie des données se fait généralement en utilisant le logiciel Epi Info, puisque le processus de saisie des données est assez simple et le logiciel gratuit.'

Une fois dans l'ordinateur, les données contiendront une variable pour chaque question ou une coche sur les questionnaires. Chacune de ces variables aura un nom différent qui peut être utilisé dans l'analyse. Par exemple, la variable correspondant à la première question de l'interview du personnel pourrait être appelée "S1." L'attribution des noms de variables revient entièrement au chercheur $f$ n'importe quel nom pourra être utilisé pourvu qu'il soit unique, et compréhensible pour les gens qui utiliseront les données.

Les questionnaires de ce livre ont des noms de variables proposées, présentées entre accolades \{\}, pour chaque question ou coche. Tous les cinq questionnaires ont en commun quelques variables d'identification sur les premières pages telles que le code du centre de santé, le code du district, le type de PPS, de secteur, etc. On a donné à ces variables des noms intuitifs tels que \{CODEDIST\}, \{DATE\} ou \{TYPE\}. En plus, chaque questionnaire a une variable d'identification appelée $\{$ IID $\},\{O I D\},\{I D\},\{P I D\}$, et $\{$ SID $\}$, pour représenter les variables d'identification de l'inventaire, de l'observation, de l'interview de sortie de la cliente PF, de l'interview du personnel et de l'interview de la cliente SMI respectivement. Ces variables portent les numéros des questionnaires décrits ci-dessus.

Le reste des noms de variables de chaque questionnaire suit un modèle simple. Ils commencent tous par une lettre : "I" pour l'inventaire, "O" pour l'observation, "F" pour l'inventaire de fin de sortie de la cliente PF, "P" pour l'interview du personnel, ou "S" pour l'interview de sortie de la cliente SMI, pour identifier le module. Vient ensuite le numéro du questionnaire, qui va de 1 à plus de 100 dans l'interview de sortie de la cliente PF. Si la question est composée de plusieurs parties, telles que plusieurs lignes dans un tableau dont chacune peut être cochée séparément, alors chaque partie est considérée comme étant une "extension" de cette question. Les extensions sont marquées par un " $\mathrm{X}$ " et un numéro d'extension. Par exemple, la

\footnotetext{
' Epi Info est un programme épidémiologique fonctionnant sous DOS qui permet la conception des questionnaires, la saisie des données et l'analyse des données. Il a été développé par le Centers for Disease Control. Il est disponible gratuitement auprès de la Division de la Surveillance et de l'Epidémiologie, Bureau du Programme d'Epidémiologie, Centers for Disease Control and Prevention, Atlanta, Georgia, 30033 U.S.A., téléphone (404) 728-0545. Il peut être aussi téléchargé à partir du site Web de CDC à l'adresse suivante : www.cdc.gov.
} 
question 4 de l'interview de la cliente PF demande les autres services que la cliente a reçu dans le PPS. Il y a seize autres services énumérés, et chacun d'entre eux peut être coché séparément. De ce fait, chacun d'entre eux a besoin d'un nom de variable séparée. Ces variables sont nommées : “F4X1," “F4X2," “F4X3," et ainsi de suite jusqu'à "F4X16." Ainsi, le nom de chaque variable indique au chercheur le questionnaire dont il est issu, la question sur laquelle il porte, et la partie de la question dont il traite.

Certaines des questions ont des listes de réponses possibles, comme c'est le cas de l'exemple ci-dessus, et un espace pour "Autre :

." Dans ces cas, l'interviewer écrit la catégorie spécifique et la coche. Cette information écrite sur "autre" doit être enregistrée aussi dans les données, et a besoin d'une autre catégorie séparée de variables. Ces variables sont nommées avec un " $R$ " à la fin pour représenter la rubrique autre. ${ }^{3}$ Dans la question 4 de l'interview de sortie de la cliente $\mathrm{PF}$, par exemple, la dernière extension est "Autre : " et l'interviewer coche si cet "autre" service a été reçu. Le nom de variable pour la coche est "F4X16," et le nom de la variable pour l'information écrite dans la ligne est "F4X16R."

Ce système de suggestion de nom de variable peut paraître complexe à première vue, mais des nombreux systèmes que l'on a essayés avec les études d'AS, celui-ci s'est avéré de loin le système le plus facile à utiliser. Si les noms des variables sont créés à partir des abréviations anglaises basées sur le contenu de la question comme “OTHSVC1," l'utilisateur découvre vite que les noms des variables deviennent gênants, non intuitifs et ne sont pas uniques. Par contre, si les

\footnotetext{
${ }^{2}$ Notez que le " $\mathrm{X}$ " est une partie importante du nom de la variable et ne devrait pas être effacé. Si l'on enlevait le " $\mathrm{X}$ " des noms de ces variables, ils deviendraient "F41," et "F42" et ainsi de suite. Ceci devient un problème parce qu'on ne peut pas distinguer ces noms de ceux des questions 41 et 42 de l'interview.

${ }^{3}$ Il aurait été plus intuitif d'utiliser "O" pour "Other" en anglais, mais un " $O$ " se confond facilement avec un zéro, et les noms des variables deviennent assez difficiles avec cette approche.
}

noms des variables sont systématiquement basés sur le numéro de la question, comme "F4X1," alors, l'utilisateur peut trouver le nom de la variable en regardant simplement la question, et trouver la question en regardant simplement le nom de la variable. Ce procédé est très utile et permet de gagner du temps dans l'analyse.

Une fois que toutes les données sont saisies, elle doivent être nettoyées. Il y a deux sortes de nettoyages de base qui devraient toujours être faits avant toute analyse.

\section{- Vérification des codes erronés}

Un "Code erroné" est une valeur d'une variable qui n'a pas de sens. Par exemple, la question peut être "Avez-vous accepté la PF aujourd'hui ?" et les réponses sont " $1=$ non" et " $2=$ oui" pour 100 clientes. Après la saisie des données, la fréquence de cette variable fait ressortir 75 cas de " 1 ," 24 cas de " 2 ," et un cas “3." Puisque “3” n'est pas une réponse possible pour cette question, ça doit être une erreur de saisie des données. Ceci est appelé "code erroné." Il peut être corrigé en regardant à la variable ID pour ce cas (IID, OID, FIG, DID, ou MID), en utilisant cette méthode pour trouver le questionnaire original, et en vérifiant la question concernée. Généralement, la réponse correcte est disponible de cette manière, et le cas peut être enregistré dans " 1 "ou " 2 ."

Cette première étape consiste donc à regarder les fréquences de toutes les variables et à les vérifier par rapport aux questionnaires pour voir s'il n'y a pas de codes erronés. Si pour une raison quelconque les questionnaires originaux ne sont pas disponibles ou bien les données de cette question manquent, alors les codes erronés devraient être fixés à " 9 " ou à un autre code qui signifie "données manquantes."

\section{- Vérification de la consistance interne} Une "question filtre" fait la sélection de certaines personnes auxquelles on posera des 
questions de suivi. Un exemple simple de question filtre est : "Voulez-vous avoir davantage d'enfants ?" avec les réponses "oui" ou "non." Si la cliente répond "oui," on lui pose ensuite la question, "Quand voulezvous avoir davantage d'enfants ?" Si 25 personnes sur 100 répondent "oui" à la première question, alors 25 personnes devraient avoir une réponse pour la deuxième question. Si 25 personnes répondent "oui" à la première question, et 35 répondent à la deuxième question, alors les données ont une inconsistance interne. Les questionnaires originaux devraient être vérifiés.

La vérification de la consistance est extrêmement importante dans le questionnaire de fin de consultation de la cliente PF. D'abord, on pose des questions à toutes les clientes ensemble, et ensuite une question filtre les repartit en nouvelles et anciennes clientes, auxquelles on pose des questions spécifiques. Les clientes sont ensuite divisées en utilisatrices des différentes méthodes, auxquelles on pose un autre groupe de questions spécifiques. Pour cette raison, il est important de vérifier toutes les consistances internes de ce module en particulier.

Si les données sont saisies à l'aide de Epi Info, il est possible de créer des vérifications automatiques qui empêchent aux agents de saisie des données de saisir des codes erronés ou des inconsistances internes par erreur. Pour plus d'information, se référer au manuel de Epi Info sur la création des fichiers ayant l'extension .CHK. 


\section{Plan d'analyse}

Les résultats préliminaires obtenus de la plupart des études d'AS sont représentés dans un rapport qui donne beaucoup d'informations possibles pertinentes, simples et descriptives sur le programme. Plus tard, les données pourraient être utilisées pour une analyse secondaire approfondie, mais la première étape est une simple présentation des résultats. Au fil des années, ces rapports préliminaires ont développé une structure assez standard. Le tableau 1 donne un plan type d'un rapport d'une AS.

TABLEAU 1. Plan type pour un rapport préliminaire d'une Analyse Sitationnelle

I. Introduction
A. Historique du programme PF dans le pays/région
B. Principales agences impliquées dans l'étude d'AS
C. Justification de l'étude d'AS
D. Objectifs de l'étude d'AS

\section{Méthodologie de l'étude}

A. Méthode d'échantillonnage

B. Mise en oeuvre

i. Révision des questionnaires

ii. Formation des agents de collecte des données

iii. Processus de collecte des données

iv. Nettoyage et saisie des données

C. Analyse et utilisation des données

i. Analyse des données

ii. Diffusion des données

III. Résultats

A. Description des échantillons

B. Capacité fonctionnelle du PPS dans l'offre de services

i. Services fournis

ii. Infrastructure, installations et équipement

iii. Expérience et formation du personnel

iv. Matériels et activités d'IEC

v. Provisions et logistique

vi. Stockage des données, rédaction des rapport et supervision

C. Qualité des services
i. Relations interpersonnelles
ii. Choix des méthodes
iii. Echange d'information
iv. Compétence technique
v. Mécanisme pour encourager la continuité
vi. Constellation et acceptabilité des services

\section{Recommandation pour une prise de décision}

Appendice 1 : Liste de tous les participants/agents de collecte des données

Appendice 2 : Instruments 
Le plan d'analyse suivant traite de la portion des "résultats" du plan du Tableau 1. Elle classe l'information collectée par les instruments de l'AS en trois rubriques : description des échantillons, capacité fonctionnelle du PPS dans l'offre des services et qualité des soins. Les deux dernières parties sont divisées davantage en sous éléments. L'information sous le volet "Capacité fonctionnelle du PPS dans l'offre des services" est classée en : services fournis, infrastructure, personnel, matériels d'IEC, provisions, stockage des données et supervision. L'information sous le volet "Qualité des soins" est classée selon le modèle de Bruce/Jain décrit dans le Chapitre 1 : relations interpersonnelles, choix des méthodes, échange d'information, compétence technique, mécanisme pour encourager la continuité, et la constellation et l'acceptabilité des services.

L'information sous chacune de ces rubriques est présentée comme une liste d'indicateurs.

Chaque indicateur spécifie une distribution, un pourcentage, ou la moyenne d'un résultat, qui peut être utilisé comme une indication de la qualité des services. Certains de ces indicateurs sont assez simples et.directs, par contre d'autres nécessitent de grands calculs impliquant beaucoup de variables. La quantité d'information recueillie dans une AS peut être considérable. Ce plan d'analyse inclut l'information recueillie de presque toutes les variables de tous les cinq instruments de l'AS, donnant plus de 130 indicateurs. Cependant, aucune analyse écrite n'a utilisé tous les indicateurs énumérés. Au contraire, les chercheurs et les responsables de programmes choisissent le groupe d'indicateurs qu'ils jugent comme étant le plus important pour leur programme, et ne rédigent les rapports que sur ces derniers. Les indicateurs qui sont les plus fréquemment utilisés et qui semblent fournir le plus d'information sur le fonctionnement du programme sont marqués d'une coche $(\checkmark)$ à côté du nom de l'indicateur.

Le plan d'analyse inclut aussi l'information relative à la manière de calculer les indicateurs. Les trois colonnes centrales du tableau guident l'utilisateur vers l'instrument, la question et les variables qui fournissent l'information pour chaque indicateur. La colonne à l'extrême droite donne les commentaires sur la façon dont l'indicateur est normalement calculé et présenté. Notez que pour les calculs particulièrement difficiles ou complexes, davantage de commentaires détaillés sont fournis à la fin du tableau. 
Plan d'analyse : Description des échantillons

\begin{tabular}{|c|c|c|c|c|c|}
\hline \multicolumn{2}{|l|}{$\begin{array}{l}\text { Résultat } \\
\checkmark=\text { Indicateur fréquemment utilisé }\end{array}$} & \multirow{2}{*}{$\begin{array}{l}\text { Instr } \\
\text { Tout }\end{array}$} & \multirow{2}{*}{\begin{tabular}{|l|}
$\begin{array}{l}\text { Numéros des } \\
\text { questions }\end{array}$ \\
-
\end{tabular}} & \multirow{2}{*}{$\begin{array}{l}\text { Variables } \\
-\end{array}$} & \multirow{2}{*}{$\begin{array}{l}\text { Commentaires } \\
\text { Ceci représente le n total de chaque } \\
\text { groupe de données. Souvent présenté } \\
\text { aussi par district. }\end{array}$} \\
\hline $\begin{array}{l}\text { 1. Nombre de PPS visités et } n \\
\text { pour chaque instrument }\end{array}$ & $\checkmark$ & & & & \\
\hline 2. Répartition des PPS par type & $\checkmark$ & 1 & $\begin{array}{l}\text { Page de } \\
\text { couverture }\end{array}$ & TYPE & \multirow{3}{*}{$\begin{array}{l}\text { Ne pas utiliser les variables TYPE, SECT } \\
\text { ou LOC d'un autre instrument différent de } \\
\text { l'inventaire. }\end{array}$} \\
\hline 3. Répartition des PPS par secteur & $\checkmark$ & 1 & $\begin{array}{l}\text { Page de } \\
\text { couverture }\end{array}$ & SECT & \\
\hline 4. Répartition des PPS par localité & $\checkmark$ & 1 & $\begin{array}{l}\text { Page de } \\
\text { couverture }\end{array}$ & LOC & \\
\hline $\begin{array}{l}\text { 5. Répartition des clientes PF par } \\
\text { type. }\end{array}$ & $\checkmark$ & 0 & Page 2 & BUT & $\begin{array}{l}\text { Présentez sous forme de diagramme en } \\
\text { camembert. }\end{array}$ \\
\hline \multirow[t]{2}{*}{$\begin{array}{l}\text { 6. Caractéristiques socio- } \\
\text { économiques des clientes PF et } \\
\text { des clientes SM }\end{array}$} & \multirow[t]{2}{*}{$\checkmark$} & $\mathrm{F}$ & $\begin{array}{l}103,104,105 \\
112,113,114 \\
115\end{array}$ & $\begin{array}{l}\text { F103, F104, } \\
\text { F105, F112, } \\
\text { F113X1 à F113X5, } \\
\text { F114, F115 }\end{array}$ & \multirow{2}{*}{$\begin{array}{l}\text { Ceci inclut : l'âge, le statut matrimonial, } \\
\text { l'alphabétisme, les langues parlées, le } \\
\text { niveau d'éducation et la religion. } \\
\text { Généralement, les résultats pour les } \\
\text { clientes PF et SMI sont présentés comme } \\
\text { deux colonnes dans un tableau. }\end{array}$} \\
\hline & & & & $\begin{array}{l}\text { M51, M52, M53, } \\
\text { M60, M61X1 à } \\
\text { M61X5, M62, } \\
\text { M63 }\end{array}$ & \\
\hline \multirow[t]{2}{*}{$\begin{array}{l}\text { 7. Statut de reproduction et } \\
\text { antécédents sexuels des } \\
\text { clientes PF et de SMI }\end{array}$} & \multirow[t]{2}{*}{$\checkmark$} & $\mathrm{F}$ & $\begin{array}{l}101,102,108 \\
110,110,111 \\
116,117,124 \\
125,126\end{array}$ & $\begin{array}{l}F 101, F 102, F 108, \\
F 109, F 110, F 111, \\
F 116, F 117, F 124, \\
F 125, F 126\end{array}$ & \multirow{2}{*}{$\begin{array}{l}\text { Ces variables incluent : le nombre } \\
\text { d'enfants, l'âge du dernier né, le statut } \\
\text { d'allaitement, les intentions en matière de } \\
\text { reproduction, le nombre de partenaires au } \\
\text { cours de la dernière année, tous nouveaux } \\
\text { partenaires au cours de la dernière année, } \\
\text { si a utilisé un préservatif avec le nouveau } \\
\text { partenaire, et antécédents d'avortement. }\end{array}$} \\
\hline & & M & $\begin{array}{l}94,50,56,57 \\
58,59,64,65 \\
74,75,76\end{array}$ & $\begin{array}{l}\text { M49, M50, M56, } \\
\text { M57, M58, M59, } \\
\text { M64, M65, M74, } \\
\text { M75, M76 }\end{array}$ & \\
\hline \multirow[t]{5}{*}{$\begin{array}{l}\text { 8. Passé contraceptif des clientes } \\
\text { PF et de SMI }\end{array}$} & \multirow[t]{5}{*}{$\checkmark$} & $\mathrm{F}$ & $\begin{array}{l}43,44,62 \\
106,107\end{array}$ & $\begin{array}{l}\text { F43, } \\
\text { F44X1 à F44X98, } \\
\text { F62, F106, F107 }\end{array}$ & \multirow{5}{*}{$\begin{array}{l}\text { Celles-ci incluent: } \\
\text { Pour toutes les clientes : déjà discuté } \\
\text { de PF avec le partenaire, si le } \\
\text { partenaire est informé de l'utilisation } \\
\text { de la PF. } \\
\text { Pour les clientes SMI : Connaissance } \\
\text { et attitude par rapport à la PF, } \\
\text { utilisation actuelle. } \\
\text { Pour les nouvelles clientes PF : déjà } \\
\text { utilisé la PF. } \\
\text { Pour les anciennes clientes PF : } \\
\text { raison de vouloir arrêter ou changer }\end{array}$} \\
\hline & & M & $\begin{array}{l}39,40,44,45 \\
46,54,55\end{array}$ & $\begin{array}{l}\text { M39, } \\
\text { M40X1 à M40X13, }\end{array}$ & \\
\hline & & & & $\begin{array}{l}\text { M44, M45, M46, } \\
\text { M54, M55 }\end{array}$ & \\
\hline & & & & & \\
\hline & & & & & \\
\hline
\end{tabular}

I = Inventaire 0 = Guide d'observation $F=$ Interview de sortie de la cliente PF S = Interview du personnel $M=$ Interview de sortie de la cliente de SMI 


\section{Plan d'analyse : Description des échantillons}

\begin{tabular}{|c|c|c|c|c|c|}
\hline $\begin{array}{l}\text { Résultat } \\
\checkmark=\text { Indicateur fréquemment utilisé }\end{array}$ & & Instr & $\begin{array}{l}\text { Numéros des } \\
\text { questions }\end{array}$ & Variables & Commentaires \\
\hline $\begin{array}{l}\text { 9. Caractéristiques socio- } \\
\text { démographiques des } \\
\text { prestataires }\end{array}$ & $\checkmark$ & S & $\begin{array}{l}32,33,34,35 \\
36\end{array}$ & $\begin{array}{l}\text { S32, S33, S34, } \\
\text { S35, S36 }\end{array}$ & $\begin{array}{l}\text { Celles-ci incluent : l'âge, le statut } \\
\text { matrimonial, le nombre d'enfants, le sexe } \\
\text { et la religion }\end{array}$ \\
\hline $\begin{array}{l}\text { 10. Utilisation actuelle de la PF par } \\
\text { les prestataires }\end{array}$ & & S & 37 & S37X1 à S37X77 & $\begin{array}{l}\text { Présentez sous forme de diagramme en } \\
\text { camembert. Inclut "aucun" comme } \\
\text { partie du graphique. }\end{array}$ \\
\hline
\end{tabular}

$\mathrm{I}=$ Inventaire $\mathrm{O}=$ Guide d'observation $\mathrm{F}=$ Interview de sortie de la cliente PF $\mathrm{S}=$ Interview du personnel $\mathrm{M}=$ Interview de sortie de la cliente de SMI 
Plan d'analyse : Capacité à fournir des services

Indicateur de la capacité

$\checkmark=$ Indicateur fréquemment utilisé

\begin{tabular}{|c|c|c|}
\hline Instr & $\begin{array}{l}\text { Numéros des } \\
\text { questions }\end{array}$ & Variables \\
\hline
\end{tabular}

Services fournis

11. Pourcentage de PPS qui offrent d'habitude les méthodes de PF

12. Pourcentage de PPS qui offrent d'habitude d'autres services, soit dans l'unité de PF ou ailleurs dans cette formation sanitiaire

13. Nombre moyen et médian de nouvelles et d'anciennes clientes dans une année, par type de service

\begin{tabular}{|l|l|l|l}
\hline & $I$ & 18,21 & $\begin{array}{l}\text { I18X1 à I18X9 } \\
\text { I21X1 à I21X6 }\end{array}$ \\
\hline$\checkmark$ & 1 & 8 & $\begin{array}{l}\text { 8A2 à I8A17 } \\
\text { I8B2 à I8B17 }\end{array}$ \\
\hline & 1 & 36,37 & $\begin{array}{l}\text { I36A1 à I36A7 } \\
\text { I36B1 à I36B7 } \\
\text { I36C1 à I36C7 } \\
\text { I37X1, I37X2 }\end{array}$ \\
\hline
\end{tabular}

14. Répartition des PPS par le volume de la clientèle

15. Nombre de jours par semaine pendant lesquels les services de PF sont offerts

16. Répartition des clientes SMI entre les autres services

17. Utilisation des services par les clientes SMI, dans cette clinique et ailleurs

18. Pourcentage de PPS qui font des tests de grossesse et de maladies spécifiques

font
19. Pourcentage de PPS qui offrent généralement des vaccinations spécifiques

20. Pourcentage de prestataires rapportant que les clientes sollicitent des conseils sur l'avortement dans leur PPS

21. Pourcentage de prestataires rapportant que les clientes sollicitent des soins post avortement dans leur PPS

\section{Commentaires}

Présentez sous fourme de diagramme en bâtons par méthode

Présentez sous fourme de diagramme en bâtons par service

Si les données sont fournies pour une période de moins d'un an, il faut créer une estimation annuelle en prenant une moyenne mensuelle (à partir de la colonne $\mathrm{C}$ ), et la multiplier par 12. Utilisez la q. 37 pour vérifier le caractère récent de l'information

Divisez les PPS en quartiles par volume de la clientèle et rapportez le pourcentage du volume total de la clientèle par quartile. $^{1}$

$\mid 36 \mathrm{~A} 1$

I36B1

I36C1

Présentez comme une moyenne et/ou sous forme de diagramme en camembert.

Ceci donne une idée de l'importance relative d'autres services au programme.

Présentez sous fourme de diagramme en bâtons avec deux séries : une pour cette clinique et l'autre pour les autres PPS.

Notez que "faire" un test veut dire le faire dans le PPS même ou par référence. Présentez sous forme de diagramme en bâtons par test. Utilisez la q. 22 comme filtre.

Présentez sous forme de diagramme en bâtons

Ces questions donnent une idée générale de la demande d'avortement dans la zone. Elles ne sont que suggestives puisque le prestataire peut ne pas voir un échantillon représentatif des patientes d'avortement.

De même, de grands biais rapportés affectent ces questions, particulièrement dans les pays où l'avortement est illégal. 
Plan d'analyse : Capacité à fournir des services

\begin{tabular}{|l|l|l|l|l|l|}
\hline $\begin{array}{l}\text { Indicateur de la capacité } \\
\checkmark=\text { Indicateur fréquemment utilisé }\end{array}$ & Instr & $\begin{array}{l}\text { Numéros des } \\
\text { questions }\end{array}$ & Variables & Commentaires \\
\hline
\end{tabular}

Infrastructure, installations et équipement

\begin{tabular}{l|l|l|l|l|l}
$\begin{array}{l}\text { 22. Pourcentage de PPS disposant } \\
\text { de l'infrastructure fonctionnelle } \\
\text { nécessaire }\end{array}$ & $\checkmark$ & I & 9 & I9X1 à I9X6 & $\begin{array}{l}\text { Ceci inclut l'eau, l'électricité, les toilettes } \\
\text { et les chaises. Présentez sous forme de } \\
\text { diagramme en bâtons }\end{array}$ \\
\hline $\begin{array}{l}\text { 23. Pourcentage de PPS qui } \\
\text { disposent de lieux d'examen } \\
\text { appropriés }\end{array}$ & $\checkmark$ & 1 & 15 & $\begin{array}{l}\text { Ceci inclut: l'intimité auditive, visuelle, la } \\
\text { propreté, suffisamment de lumière, l'eau } \\
\text { en quantité suffisante. . Présentez sous } \\
\text { forme de diagramme en bâtons }\end{array}$ \\
\hline $\begin{array}{l}\text { 24. Pourcentage de PPS ne } \\
\text { disposant pas de l'équipement } \\
\text { de PF sélectionné }\end{array}$ & $\checkmark$ & 1 & 16,17 & $\begin{array}{l}\text { I16X1 à I16X18 } \\
\text { I17X1 à I17X6 }\end{array}$ & $\begin{array}{l}\text { Parmi les nombreux éléments } \\
\text { d'équipement énumérés, choisissez ceux } \\
\text { que les responsables de programme } \\
\text { jugent comme étant les plus importants. } \\
\text { Notez que cet indicateur fait appel à } \\
\text { l'inverse des données: si 85\% des PPS } \\
\text { disposent d'un stérilisateur, rapportez ici } \\
\text { donc que 15\% ne disposent pas de } \\
\text { stérilisateur. Sur les éléments comptés, } \\
\text { fixez un minimum raisonnable pour le } \\
\text { nombre d'éléments qu'un PPS devrait } \\
\text { avoir pour être bien équipé. Ensuite faites } \\
\text { un rapport sur les PPS qui ont moins du } \\
\text { minimum. }\end{array}$ \\
\hline
\end{tabular}

Expérience et formation du personnel

\begin{tabular}{l|l|l|l|l|l}
$\begin{array}{l}\text { 25. Distribution des prestataires par } \\
\text { type }\end{array}$ & I & 10 & I10X1 à I10X7 & $\begin{array}{l}\text { Présentez sous forme de diagramme en } \\
\text { camembert. Ne pas utiliser le type de } \\
\text { personnel du module d'interview du } \\
\text { personnel, mais seulement celui de } \\
\text { l'inventaire. }\end{array}$ \\
\hline $\begin{array}{l}\text { 26. Distribution des prestataires qui } \\
\text { sont de service aujourd'hui par } \\
\text { type }\end{array}$ & & I & 11 & I11X1 à I11X7 & $\begin{array}{l}\text { Comparez au total du personnel de } \\
\text { l'indicateur 25. }\end{array}$ \\
\hline $\begin{array}{l}\text { 27. Pourcentage de personnels } \\
\text { dont la formation de base a } \\
\text { inclus divers domaines }\end{array}$ & $\checkmark$ & $S$ & 4 & $S 4 X 1$ à S4X12 & $\begin{array}{l}\text { Présentez sous forme de diagramme en } \\
\text { bâtons par thème. Peut être présenté aussi } \\
\text { par type de prestataire. }\end{array}$ \\
\hline $\begin{array}{l}\text { 28. Nombre moyen et médian } \\
\text { d'années depuis la formation de } \\
\text { base }\end{array}$ & $\checkmark$ & $S$ & 3 & Vous pouvez présenter par type de \\
prestataire s'il y a une grande différence.
\end{tabular}

I = Inventaire 0 = Guide d'observation $F=$ Interview de sortie de la cliente PF $S=$ Interview du personnel $M=$ Interview de sortie de la cliente de SMI 
Plan d'analyse : Capacité à fournir des services

\begin{tabular}{|c|c|c|c|c|c|}
\hline \multicolumn{2}{|l|}{$\begin{array}{l}\text { Indicateur de la capacité } \\
\checkmark=\text { Indicateur fréquemment utilisé }\end{array}$} & \multirow{2}{*}{\begin{tabular}{|l} 
Instr \\
S
\end{tabular}} & \multirow{2}{*}{\begin{tabular}{|l}
$\begin{array}{l}\text { Numéros des } \\
\text { questions }\end{array}$ \\
6,7
\end{tabular}} & \multirow{2}{*}{\begin{tabular}{|l} 
Variables \\
S6, \\
S7X1 à S7X19
\end{tabular}} & \multirow{2}{*}{\begin{tabular}{|l|} 
Commentaires \\
Présentez sous forme de diagramme en \\
bâtons par thème. Peut faire un rapport à \\
tout le personnel, ou utiliser la q.6 comme \\
filtre et rapporter aux seuls personnels qui \\
ont reçu cette formation. \\
\end{tabular}} \\
\hline $\begin{array}{l}\text { 29. Pourcentage de prestataires } \\
\text { dont la formation continue/de } \\
\text { recyclage a inclus des } \\
\text { questions spécifiques de } \\
\text { PF/MST }\end{array}$ & $\checkmark$ & & & & \\
\hline $\begin{array}{l}\text { 30. Nombre moyen et médian } \\
\text { d'années depuis la formation } \\
\text { continue/de recyclage dans les } \\
\text { questions relatives à la PF/MST }\end{array}$ & & $S$ & 8 & S8X1 à S8X19 & $\begin{array}{l}\text { Calculez ceci par thème, pour les } \\
\text { personnels rapportant à la q.8. Notez que } \\
\text { le n va changer pour chaque thème. }\end{array}$ \\
\hline $\begin{array}{l}\text { 31. Pourcentage de prestataires qui } \\
\text { ont suivi un recyclage dans } \\
\text { d'autres domaines de la santé }\end{array}$ & $\checkmark$ & S & 5 & S5X2 à S5X12 & $\begin{array}{l}\text { Présentez sous forme de diagramme en } \\
\text { bâtons. }\end{array}$ \\
\hline $\begin{array}{l}\text { 32. Nombre moyen et médian } \\
\text { d'années depuis que les } \\
\text { prestataires travaillent dans ce } \\
\text { PPS } \\
\end{array}$ & & S & 2 & S2 & $\begin{array}{l}\text { Vous pouvez présenter par type de } \\
\text { personnel s'il y a une grande différence. }\end{array}$ \\
\hline $\begin{array}{l}\text { 33. Pourcentage de prestataires } \\
\text { fournissant activement divers } \\
\text { services dans ce PPS }\end{array}$ & & S & 1 & S1X1 à S1X18 & $\begin{array}{l}\text { Présentez sous forme de diagramme en } \\
\text { bâtons. }\end{array}$ \\
\hline $\begin{array}{l}\text { 34. Pourcentage de prestataires } \\
\text { ayant fourni diverses méthodes } \\
\text { PF au cours des } 3 \text { derniers } \\
\text { mois }\end{array}$ & & S & 9,10 & $\begin{array}{l}\text { S9 } \\
\text { S10X1 à S10X15 }\end{array}$ & $\begin{array}{l}\text { Présentez sous forme de diagramme en } \\
\text { bâtons. Se calcule généralement avec tout } \\
\text { le personnel; ne pas utiliser la q.9 comme } \\
\text { filtre. }\end{array}$ \\
\hline
\end{tabular}

\section{Matériels et activités d'IEC}

\begin{tabular}{l|l|l|l|l|l}
$\begin{array}{l}\text { 35. Pourcentage de PPS avec un } \\
\text { panneau annonçant les services } \\
\text { de PF }\end{array}$ & & I & 7 & 17 & $\begin{array}{l}\text { IPrésentez sous forme de diagramme en } \\
\text { camembert. }\end{array}$ \\
\hline $\begin{array}{l}\text { 36. Pourcentage de PPS avec } \\
\text { divers matériels d'IEC } \\
\text { disponibles }\end{array}$ & $\checkmark$ & $I$ & 12 & $\begin{array}{l}\text { I12A1 à I12A7 } \\
\text { I12B1 à I12B7 } \\
\text { I12C1 à I12C7 }\end{array}$ & $\begin{array}{l}\text { Présentez sous forme de diagramme en } \\
\text { tableau ou en bâtons avec les éléments } \\
\text { d'IEC sur l'axe des X et une barre } \\
\text { différente pour chaque thème. }\end{array}$ \\
\hline $\begin{array}{l}\text { 37. Pourcentage de PPS qui } \\
\text { organisent des causeries } \\
\text { sanitaires }\end{array}$ & $\checkmark$ & $I$ & 13 & I13 & \\
\hline $\begin{array}{l}\text { 38. Pourcentage de causeries } \\
\text { sanitaires qui incluent divers } \\
\text { thèmes }\end{array}$ & $\checkmark$ & $I$ & 14 & I14X1 à I14X15 & $\begin{array}{l}\text { Présentez sous forme de diagramme en } \\
\text { bâtons. Utilisez la q.13 comme filtre. }\end{array}$ \\
\hline
\end{tabular}

$\mathrm{I}=$ Inventaire $\mathrm{O}=$ Guide d'observation $\mathrm{F}=$ Interview de sortie de la cliente PF $\mathrm{S}=$ Interview du personnel $\mathrm{M}=$ Interview de sortie de la cliente de SMI 


\section{Plan d'analyse : Capacité à fournir des services}

\begin{tabular}{|l|l|l|l|l|l|}
\hline $\begin{array}{l}\text { Indicateur de la capacité } \\
V=\text { Indicateur fréquemment utilisé }\end{array}$ & Instr & $\begin{array}{l}\text { Numéros des } \\
\text { questions }\end{array}$ & Variables & Commentaires \\
\hline
\end{tabular}

Approvisionnement et logistique

39. Pourcentage de PPS connaissant des ruptures de stocks de produits contraceptifs au cours des six derniers mois.

\begin{tabular}{|l|l|l|l}
$\checkmark$ & $I$ & $18,19,20$ & $\begin{array}{l}\text { I18X1 à I18X9 } \\
\text { I19X1 à I19X9 } \\
\text { I20X1 à I20X9 }\end{array}$ \\
\hline
\end{tabular}

Si le PPS a connu une rupture de stock au cours des six derniers mois (q.20) ou ne dispose pas de provisions aujourd'hui (q.19), il faut le compter comme ayant connu une rupture de stock. Utilisez la q. 18 comme filtre - ne pas inclure dans le dénominateur les PPS qui n'offrent pas la méthode particulière. Le $n$ changera pour chaque méthode. \begin{tabular}{ll|l|l|l|l|l}
\hline $\begin{array}{l}\text { 40. Pourcentage de PPS disposant } \\
\text { de conditions de stockage }\end{array}$ & $\checkmark$ & 1 & 29 & I29X1 à I29X4 & $\begin{array}{l}\text { Présentez sous forme de diagramme en } \\
\text { bâtons par produit. }\end{array}$
\end{tabular} appropriées pour les produits

41. Pourcentage de PPS qui stocke les produits contraceptifs selon la date de péremption

42. Pourcentage de PPS ayant des inventaires de stocks

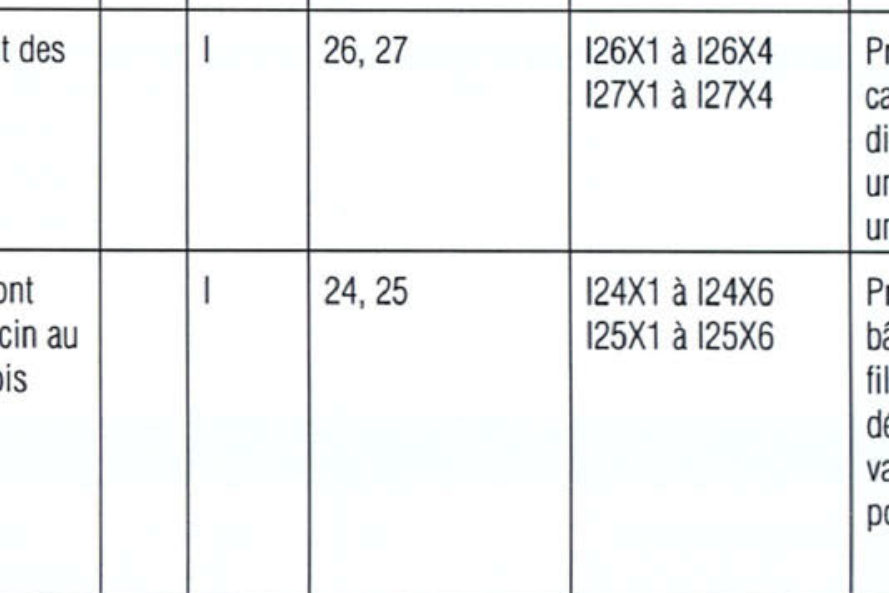

Présentez sous forme de diagramme en camembert avec trois tranches: PPS ne disposant pas d'inventaires, ceux qui ont un inventaire en bon état, et ceux qui ont un inventaire qui n'est pas en bon état.

43. Pourcentage de PPS qui ont connu une rupture de vaccin au cours des six derniers mois

Présentez sous forme de diagramme en bâtons par produit. Utilisez la q.24 comme filtre - ne pas inclure dans le dénominateur les PPS qui n'offrent pas la vaccination particulière. Le $\mathrm{n}$ changera pour chaque vaccin.

Stockage des donées, rédaction des rapports et supervision

\begin{tabular}{l|l|l|l|l|l}
$\begin{array}{l}\text { 44. Pourcentage de PPS ayant des } \\
\text { systèmes de fiches de clientes }\end{array}$ & $\checkmark$ & I & 30 & I30 & $\begin{array}{l}\text { Présentez sous forme de diagramme en } \\
\text { camembert. }\end{array}$ \\
\hline $\begin{array}{l}\text { 45. De ces PPS ayant des systèmes } \\
\text { de fiches de clientes, } \\
\text { pourcentage ayant des fiches en } \\
\text { bon état }\end{array}$ & $\checkmark$ & $I$ & 30,31 & 130,131 & $\begin{array}{l}\text { Présentez sous forme de diagramme en } \\
\text { camembert. Utilisez la q.30 comme filtre. }\end{array}$ \\
\hline $\begin{array}{l}\text { 46. Pourcentage de PPS disposant } \\
\text { de registres d'activités } \\
\text { quotidiennes }\end{array}$ & & 1 & 32 & 132 & $\begin{array}{l}\text { Présentez sous forme de diagramme en } \\
\text { camembert. }\end{array}$ \\
\hline $\begin{array}{l}\text { 47. Pourcentage de PPS qui } \\
\text { envoient des rapports } \\
\text { statistiques à des unités } \\
\text { supérieures }\end{array}$ & & 1 & 33 & I33X1 à I33X3 & $\begin{array}{l}\text { Présentez sous forme de diagramme en } \\
\text { bâtons par type de service. }\end{array}$ \\
\hline
\end{tabular}

$I=$ Inventaire 0 = Guide d'observation $F=$ Interview de sortie de la cliente PF $S=$ Interview du personnel $M=$ Interview de sortie de la cliente de SMI 
Plan d'analyse : Capacité à fournir des services

\begin{tabular}{|l|l|l|l|l|l|}
\hline \multicolumn{2}{|l|}{$\begin{array}{l}\text { Indicateur de la capacité } \\
\checkmark=\text { Indicateur fréquemment utilisé }\end{array}$} & Instr & $\begin{array}{l}\text { Numéros des } \\
\text { questions }\end{array}$ & Variables & Commentaires \\
$\begin{array}{l}\text { 48. Pourcentage de PPS ayant reçu } \\
\text { au moins une visite de } \\
\begin{array}{l}\text { supervision au cours des six } \\
\text { derniers mois }\end{array}\end{array}$ & $\checkmark$ & $I$ & 34 & 134 & $\begin{array}{l}\text { Les programmes établissent souvent des } \\
\text { normes pour la fréquence des visites de } \\
\text { supervision. La formulation de cet } \\
\text { indicateur peut être changée pour voir si } \\
\text { ces normes de programme sont } \\
\text { respectées. Au lieu de "au moins une," on } \\
\text { pourrait lire "deux ou plus," etc. }\end{array}$ \\
\hline $\begin{array}{l}\text { 49. Parmi les PPS qui ont reçu une } \\
\text { visite de supervision au cours } \\
\text { des six derniers mois, nombre } \\
\text { moyen de visites par PPS }\end{array}$ & $\checkmark$ & 1 & 34 & Moyenne de seulement ceux qui ont \\
\hline $\begin{array}{l}\text { 50. Pourcentage de superviseurs } \\
\text { exécutant diverses activités }\end{array}$ & $\checkmark$ & 1 & 34,35 & I35X1 à I35X7 & $\begin{array}{l}\text { Notez le n réduit - utilisez seulement les } \\
\text { PPS qui ont eu au moins une visite de } \\
\text { supervision. } \\
\text { Présentez sous forme de diagramme en } \\
\text { bâtons. }\end{array}$ \\
\hline
\end{tabular}

$I=$ Inventaire 0 = Guide d'observation $F=$ Interview de sortie de la cliente PF $S=$ Interview du personnel $M=$ Interview de sortie de la cliente de SMI 
Plan d'analyse : Qualité des soins

\begin{tabular}{|l|l|l|l|l|l|}
\hline $\begin{array}{l}\text { Indicateur de la capacité } \\
\checkmark=\text { Indicateur fréquemment utilisé }\end{array}$ & Instr & $\begin{array}{l}\text { Numéros des } \\
\text { questions }\end{array}$ & Variables & Commentaires \\
\hline
\end{tabular}

Relations interpersonnelles

\begin{tabular}{c|l|l|l|l|l}
$\begin{array}{l}\text { 51. Pourcentage de clientes PF qui } \\
\text { ont reçu une salutation cordiale }\end{array}$ & $\checkmark$ & 0 & 1 & 01 & \\
\hline $\begin{array}{l}\text { 52. Pourcentage de clientes PF qui } \\
\text { ont le sentiment que le } \\
\text { prestataire a prêté une oreille } \\
\text { attentive à leurs inquiétudes }\end{array}$ & $\checkmark$ & $F$ & 7,8 & $F 7, F 8$ & $\begin{array}{l}\text { Rapportez soit à toutes les clientes soit à } \\
\text { celles qui avaient des inquiétudes. }\end{array}$ \\
\hline $\begin{array}{l}\text { 53. Pourcentage de clientes PF a) } \\
\text { qui avaient des questions, b) } \\
\text { que le prestataire à laissé poser } \\
\text { des questions, c) qui ont été } \\
\text { satisfaites de la réponse } \\
\text { donnée. }\end{array}$ & $\checkmark$ & $F$ & $9,10,11$ & F9, F10, F11 & $\begin{array}{l}\text { Notez le n réduit pour chaque partie de cet } \\
\text { indicateur. Ceci n'est souvent rapporté que } \\
\text { pour les nouvelles clientes. } \\
\text { Ces résultats peuvent aussi être comparés }\end{array}$ \\
\hline $\begin{array}{l}\text { 54. Pourcentage de prestataires qui } \\
\text { se sentent à l'aise en discutant } \\
\text { de VHI/SIDA }\end{array}$ & à ceux des clientes SMI (interview SMI \\
q.8,9,10).
\end{tabular}

Choix des méthodes

\begin{tabular}{|c|c|c|c|c|c|c|}
\hline 56. & $\begin{array}{l}\text { Pourcentage de nouvelles } \\
\text { clientes informées de diverses } \\
\text { méthodes }\end{array}$ & $\checkmark$ & 0 & 12 & $012 \times 1$ à $012 \times 13$ & $\begin{array}{l}\text { Présentez sous forme de diagramme en } \\
\text { bâtons par méthode. }\end{array}$ \\
\hline 57. & $\begin{array}{l}\text { Nombre moyen de méthodes } \\
\text { mentionnées aux nouvelles } \\
\text { clientes PF }\end{array}$ & $\checkmark$ & 0 & 12 & $012 \times 1$ à $012 \times 13$ & \\
\hline 58. & $\begin{array}{l}\text { Pourcentage de nouvelles } \\
\text { clientes PF et d'anciennes } \\
\text { clientes qui changent de } \\
\text { méthode et qui rapportent } \\
\text { qu'elles ont entendu parler de } \\
\text { plus d'une méthode de la part } \\
\text { du prestataire }\end{array}$ & & $F$ & 48,68 & F48, F68 & $\begin{array}{l}\text { Combinez les nouvelles clientes et les } \\
\text { anciennes qui changent de méthodes }\end{array}$ \\
\hline 59. & $\begin{array}{l}\text { Pourcentage de nouvelles } \\
\text { clientes PF à qui il a été } \\
\text { demandé si elles avaient une } \\
\text { préférence pour une méthode }\end{array}$ & $\checkmark$ & 0 & 10 & 010 & \\
\hline
\end{tabular}

$I=$ Inventaire $\quad 0$ = Guide d'observation $F=$ Interview de sortie de la cliente PF $S=$ Interview du personnel $M=$ Interview de sortie de la cliente de SMI 
Plan d'analyse : Qualité des soins

\begin{tabular}{|c|c|c|c|c|c|}
\hline $\begin{array}{l}\text { Indicateur de la capacité } \\
\checkmark=\text { Indicateur fréquemment utilisé }\end{array}$ & & Instr & $\begin{array}{l}\text { Numéros des } \\
\text { questions }\end{array}$ & Variables & Commentaires \\
\hline $\begin{array}{l}\text { 60. Pourcentage de prestataires qui } \\
\text { encouragent une méthode par } \\
\text { rapport aux autres pendant la } \\
\text { consultation } \\
\end{array}$ & & 0 & 15 & 015 & \\
\hline $\begin{array}{l}\text { 61. Pourcentage de prestataires qui } \\
\text { rapportent qu'ils imposent des } \\
\text { barrières sur les méthodes }\end{array}$ & $\checkmark$ & $S$ & $\begin{array}{l}11,12,13,14 \\
15,16,17,18\end{array}$ & $\begin{array}{l}S 11 \times 1 \text { aे } S 11 \times 5 \\
S 12 \times 1 \text { à } S 12 \times 5 \\
S 13 \times 1 \text { à } S 13 \times 5 \\
S 14 \times 1 \text { à } S 14 \times 5 \\
S 15 \times 1 \text { à } S 15 \times 5 \\
S 16 \times 1 \text { à } S 16 \times 5 \\
S 17 X 1 \text { à } S 17 \times 5 \\
S 18 X 1 \text { à } S 18 X 5\end{array}$ & $\begin{array}{l}\text { Ces barrières sont : l'âge, le statut } \\
\text { matrimonial, la parité, le consentement de } \\
\text { l'époux. Ces indicateurs nécessitent } \\
\text { beaucoup de calculs. } 2 \text { Présentez sous } \\
\text { forme de diagramme en tableau, par } \\
\text { méthode. }\end{array}$ \\
\hline $\begin{array}{l}\text { 62. Parmi les prestataires qui } \\
\text { imposent des barrières, } \\
\text { moyenne de l'âge minimum, } \\
\text { l'âge maximum et parité requis, } \\
\text { par méthode }\end{array}$ & $\checkmark$ & S & $\begin{array}{l}11,12,13,14 \\
15,16\end{array}$ & $\begin{array}{l}\text { S11X1 à S11X5 } \\
\text { S12X1 à S12X5 } \\
\text { S13X1 à S13X5 } \\
\text { S14X1 à S14X5 } \\
\text { S15X1 à S15X5 } \\
\text { S16X1 à S16X5 }\end{array}$ & $\begin{array}{l}\text { Ces indicateurs nécessitent beaucoup de } \\
\text { calculs.2 Présentez sous forme de } \\
\text { diagramme en tableau, par méthode. }\end{array}$ \\
\hline $\begin{array}{l}\text { 63. Pourcentage de prestataires qui } \\
\text { recommanderaient diverses } \\
\text { méthodes pour les clientes qui } \\
\text { veulent espacer/limiter }\end{array}$ & & S & $19,19 a$ 20, 20a & $\begin{array}{l}\text { S19, S20 } \\
\text { S19AX1 à } \\
\text { S19AX13 } \\
\text { S20AX1 à } \\
\text { S20AX13 }\end{array}$ & $\begin{array}{l}\text { Présentez sous forme de diagramme en } \\
\text { tableau, par méthode. Utilisez deux barres } \\
\text { par méthode : une pour les } \\
\text { recommandations pour les clientes qui } \\
\text { espacent, l'autre pour les } \\
\text { recommandations des clientes qui } \\
\text { limitent. }\end{array}$ \\
\hline $\begin{array}{l}\text { 64. Pourcentage de prestataires qui } \\
\text { rapportent qu'ils ne } \\
\text { recommanderaient jamais } \\
\text { certaines méthodes }\end{array}$ & $\checkmark$ & S & $22,22 a$ & $\begin{array}{l}\text { S22 } \\
\text { S22AX1 à } \\
\quad \text { S22AX13 }\end{array}$ & \\
\hline $\begin{array}{l}\text { 65. Pourcentage de clientes PF } \\
\text { dont la méthode choisie a été la } \\
\text { même que celle pour laquelle } \\
\text { elles avaient exprimé une } \\
\text { préférence } \\
\end{array}$ & & 0 & 26 & 026 & $\begin{array}{l}\text { Notez que ces deux indicateurs mesurent } \\
\text { des résultats très similaires, mais l'un } \\
\text { provient de l'observation et l'autre de } \\
\text { l'interview de sortie de la cliente. }\end{array}$ \\
\hline $\begin{array}{l}\text { 66. Pourcentage de nouvelles } \\
\text { clientes PF qui rapportent } \\
\text { avoir reçu leur méthode de } \\
\text { choix }\end{array}$ & $\checkmark$ & $\mathrm{F}$ & 48 & F48 & \\
\hline $\begin{array}{l}\text { 67. Pour les nouvelles clientes PF } \\
\text { qui n'ont pas reçu leur méthode } \\
\text { de choix, quelle est la méthode } \\
\text { préférée? }\end{array}$ & & $\mathrm{F}$ & 51,52 & F51, F52 & $\begin{array}{l}\text { Présentez sous forme de diagramme en } \\
\text { camembert. Utilisez la q. } 51 \text { comme filtre. }\end{array}$ \\
\hline
\end{tabular}

$\mathrm{I}=$ Inventaire $\mathrm{O}=$ Guide d'observation $\mathrm{F}=$ Interview de sortie de la cliente $\mathrm{PF} S=$ Interview du personnel $\mathrm{M}=$ Interview de sortie de la cliente de SMI 
Plan d'analyse : Qualité des soins

\begin{tabular}{|c|c|c|c|c|c|c|}
\hline \multicolumn{3}{|c|}{$\begin{array}{l}\text { Indicateur de la capacité } \\
\checkmark=\text { Indicateur fréquemment utilisé }\end{array}$} & \multirow{2}{*}{\begin{tabular}{|l} 
Instr \\
0
\end{tabular}} & \multirow{2}{*}{$\begin{array}{l}\text { Numéros des } \\
\text { questions } \\
26,27\end{array}$} & \multirow{2}{*}{\begin{tabular}{|l|} 
Variables \\
026,027
\end{tabular}} & \multirow{3}{*}{\begin{tabular}{|l} 
Commentaires \\
Notez que ces deux indicateurs mesurent \\
des résultats très similaires, mais l'un \\
provient de l'observation et l'autre de \\
l'interview de sortie de la cliente \\
Utilisez les q.26 et 51 comme filtres.
\end{tabular}} \\
\hline & $\begin{array}{l}\text { Pour les clientes PF dont la } \\
\text { méthode choisie n'était pas leur } \\
\text { méthode préférée, quelle est la } \\
\text { raison observée? }\end{array}$ & & & & & \\
\hline & $\begin{array}{l}\text { Pour les nouvelles clientes PF } \\
\text { qui n'ont pas reçu leur méthode } \\
\text { de choix, quelle est la raison } \\
\text { rapportée? }\end{array}$ & & $\mathrm{F}$ & 51,53 & $\begin{array}{l}\text { F51, } \\
\text { F53X1 à F53X98 }\end{array}$ & \\
\hline \multirow{2}{*}{\multicolumn{2}{|c|}{$\begin{array}{l}\text { 70. Pour les nouvelles clientes PF } \\
\text { qui ont décidé de ne pas } \\
\text { utiliser la PF, quelles sont les } \\
\text { raisons? }\end{array}$}} & & 0 & 21,22 & 021,022 & \multirow{2}{*}{$\begin{array}{l}\text { Présentez sous forme de graphiques } \\
\text { sectoriels. Utilisez les q.21 et } 45 \text { comme } \\
\text { filtres. La comparaison entre l'observation } \\
\text { et l'information de l'interview de sortie } \\
\text { donne une idée de la façon dont ces } \\
\text { décisions sont perçues par les clientes } \\
\text { par rapport aux prestataires. }\end{array}$} \\
\hline & & & $\mathrm{F}$ & 45,46 & $\mathrm{~F} 45, \mathrm{~F} 46$ & \\
\hline & $\begin{array}{l}\text { Pour les anciennes clientes PF } \\
\text { qui veulent arrêter ou changer, } \\
\text { quelles sont les raisons pour } \\
\text { arrêter ou changer? }\end{array}$ & & 0 & 23,24 & & $\begin{array}{l}\text { Présentez sous forme de graphiques } \\
\text { sectoriels. Utilisez la q. } 23 \text { comme filtre. }\end{array}$ \\
\hline \multicolumn{7}{|c|}{ Echange d'information } \\
\hline 72. & $\begin{array}{l}\text { Pourcentage de clientes PF } \\
\text { avec lesquelles on a discuté de } \\
\text { diverses questions de PF }\end{array}$ & $\checkmark$ & 0 & $2,5,7$ & $\begin{array}{l}02 \times 1 \mathrm{~A} 02 \times 77 \\
05,07\end{array}$ & $\begin{array}{l}\text { Séparez les nouvelles clientes des } \\
\text { anciennes puisqu'une discussion plus } \\
\text { complète est généralement nécessaire } \\
\text { pour les nouvelles clientes. Notez que la } \\
\text { q.5 est seulement pour les nouvelles } \\
\text { clientes, et la q.7 est seulement pour les } \\
\text { anciennes clientes. }\end{array}$ \\
\hline & $\begin{array}{l}\text { Pourcentage de clientes PF qui } \\
\text { ont reçu des informations sur la } \\
\text { méthode acceptée }\end{array}$ & $\checkmark$ & 0 & 13 & $013 \times 1$ à $013 \times 77$ & $\begin{array}{l}\text { Ceci peut être calculé pour les nouvelles } \\
\text { utilisatrices et pour les anciennes clientes } \\
\text { qui changent pour une nouvelle méthode. } \\
\text { Présentez sous forme de diagramme en } \\
\text { bâtons, par type d'information. }\end{array}$ \\
\hline & $\begin{array}{l}\text { Pourcentage de nouvelles } \\
\text { clientes PF et d'anciennes } \\
\text { clientes qui changent de } \\
\text { méthode et qui rapportent avoir } \\
\text { été informées qu'elles } \\
\text { pouvaient changer de méthodes } \\
\text { si elles n'étaient pas satisfaites } \\
\text { de leur nouvelle méthode }\end{array}$ & & $F$ & 49,69 & F49, F69 & $\begin{array}{l}\text { Combinez les nouvelles clientes et les } \\
\text { anciennes clientes qui changent de } \\
\text { méthodes. }\end{array}$ \\
\hline
\end{tabular}

$I=$ Inventaire 0 = Guide d'observation $F=$ Interview de sortie de la cliente PF S = Interview du personnel $M=$ Interview de sortie de la cliente de SMI 
Plan d'analyse : Qualité des soins

\begin{tabular}{|c|c|c|c|c|c|}
\hline \multicolumn{2}{|l|}{$\begin{array}{l}\text { Indicateur de la qualité } \\
\checkmark=\text { Indicateur fréquemment utilisé }\end{array}$} & \multirow{2}{*}{\begin{tabular}{|l} 
Instr \\
0
\end{tabular}} & \multirow{2}{*}{$\begin{array}{l}\text { Numéros des } \\
\text { questions } \\
20\end{array}$} & \multirow{2}{*}{$\begin{array}{l}\text { Variables } \\
020 \times 1 \text { à } 020 \times 77\end{array}$} & \multirow{2}{*}{\begin{tabular}{|l|} 
Commentaires \\
Présentez sous forme de diagramme en \\
bâtons, par thème. Ceci est souvent \\
rapporté séparément pour les nouvelles \\
clientes et pour les anciennes clientes. \\
\end{tabular}} \\
\hline $\begin{array}{l}\text { 75. Pourcentage de clientes PF } \\
\text { avec lesquelles on a discuté } \\
\text { d'autres services de santé }\end{array}$ & $\checkmark$ & & & & \\
\hline \multirow{2}{*}{$\begin{array}{l}\text { 76. Pourcentage de clientes PF } \\
\text { avec lesquelles des matériels } \\
\text { d'IEC ont été utilisés (dans les } \\
\text { PPS disposant de matériels } \\
\text { d'IEC disponibles) }\end{array}$} & \multirow[t]{2}{*}{$\checkmark$} & 0 & 16 & 016X1 à 016X77 & \multirow{2}{*}{$\begin{array}{l}\text { Ne calculez ceci que pour les PPS } \\
\text { disposant de matériels d'IEC, de sorte que } \\
\text { l'indicateur mesure l'utilisation des } \\
\text { matériels d'IEC plutôt que leur présence, } \\
\text { ce qui est mesuré dans l'indicateur } 36 . \\
\text { Cela nécessite que les données de } \\
\text { l'observation soient liées à l'inventaire. } \\
\end{array}$} \\
\hline & & I & 12 & $\begin{array}{l}\mid 12 A 1 \text { à } \mid 12 A 7 \\
\mid 12 B 1 \text { à } \mid 12 B 7 \\
\mid 12 C 1 \text { à } \mid 12 C 7\end{array}$ & \\
\hline \multirow{3}{*}{$\begin{array}{l}\text { 77. Pourcentage de clientes PF } \\
\text { auxquelles on a donné des } \\
\text { dépliants sur divers thèmes } \\
\text { (dans les PPS qui disposent de } \\
\text { dépliants) }\end{array}$} & & $\mathrm{F}$ & 18,19 & $\begin{array}{l}\text { F18, } \\
\text { F19X1 à F19X98 }\end{array}$ & \multirow{3}{*}{$\begin{array}{l}\text { Ne calculer ceci que pour les PPS qui ont } \\
\text { des dépliants disponibles, de sorte que } \\
\text { l'indicateur mesure la distribution des } \\
\text { matériels IEC plutôt que leur présence, ce } \\
\text { qui est mesuré dans l'indicateur } 36 \text {. Ceci } \\
\text { nécessite que les données de } \\
\text { l'observation soient liées à l'inventaire. } \\
\text { Ces résultats peuvent être comparés aussi } \\
\text { à ceux des clientes SMI (interview SMI } \\
\text { q.22,23). }\end{array}$} \\
\hline & & I & 12 & |12B1 à |12B7 & \\
\hline & & & & & \\
\hline $\begin{array}{l}\text { 78. Pourcentage de clientes PF qui } \\
\text { ont été informées que le } \\
\text { préservatif protège contre les } \\
\text { MST }\end{array}$ & $\checkmark$ & 0 & 17 & 017 & \\
\hline $\begin{array}{l}\text { 79. Pourcentage de clientes } \\
\text { utilisatrices de la MAMA } \\
\text { auxquelles on a donné des } \\
\text { informations correctes sur la } \\
\text { méthode }\end{array}$ & & 0 & 14,25 & $\begin{array}{l}025 \\
014 \times 1 \text { à } 014 \times 77\end{array}$ & $\begin{array}{l}\text { Calculez seulement pour les clientes de la } \\
\text { MAMA. Utilisez la q. } 25 \text { comme filtre. }\end{array}$ \\
\hline $\begin{array}{l}\text { 80. Pourcentage de clientes PF qui } \\
\text { ont pris part à une causerie de } \\
\text { groupe }\end{array}$ & & $F$ & 20 & $\mathrm{~F} 20$ & $\begin{array}{l}\text { Ces résultats peuvent être comparés aussi } \\
\text { à ceux des clientes SMI (interview SMI } \\
\text { q.24). }\end{array}$ \\
\hline $\begin{array}{l}\text { 81. Pourcentage de clientes PF qui } \\
\text { ont rapporté avoir entendu } \\
\text { parler de divers thèmes pendant } \\
\text { les causeries de groupe }\end{array}$ & & $F$ & 21 & F21X1 à F21X98 & $\begin{array}{l}\text { Présentez sous forme de diagramme en } \\
\text { bâtons. Si l'interview de sortie de la } \\
\text { cliente PF est liée à l'inventaire, } 3 \text { alors } \\
\text { vous pouvez comparer ce qui a été } \\
\text { raconté aux clientes pendant ces causeries } \\
\text { sanitaires avec ce qu'elles ont retenu. Voir } \\
\text { l'indicateur } 37 . \\
\text { Ces résultats peuvent être comparés aussi } \\
\text { à ceux des clientes SMI (interview SMI } \\
\text { q.25). }\end{array}$ \\
\hline
\end{tabular}

$\mathrm{I}=$ Inventaire $\mathrm{O}$ = Guide d'observation $\mathrm{F}=$ Interview de sortie de la cliente PF $\mathrm{S}=$ Interview du personnel $\mathrm{M}=$ Interview de sortie de la cliente de SMI 
Plan d'analyse : Qualité des soins

\begin{tabular}{|c|c|c|c|c|c|}
\hline \multicolumn{2}{|l|}{$\begin{array}{l}\text { Indicateur de la qualité } \\
\checkmark=\text { Indicateur fréquemment utilisé }\end{array}$} & \multirow{2}{*}{\begin{tabular}{|l|} 
Instr \\
F
\end{tabular}} & \multirow{2}{*}{\begin{tabular}{|l|}
$\begin{array}{l}\text { Numéros des } \\
\text { questions }\end{array}$ \\
pilule : $72-77$ \\
\end{tabular}} & \multirow{2}{*}{\begin{tabular}{|l} 
Variables \\
F72,F73,F74,F75 \\
F76X1 à F76X98 \\
F77X1 à F77X98
\end{tabular}} & \multirow{3}{*}{\begin{tabular}{|l} 
Commentaires \\
Ces indicateurs peuvent être rapportés \\
comme un groupe d'éléments que les \\
clientes connaissent, ou bien ils peuvent \\
être combinés en scores de \\
connaissance. ${ }^{4}$ Les résultats sont \\
généralement rapportés par méthode, \\
mais ils peuvent être rapportés aussi \\
d'une manière globale.
\end{tabular}} \\
\hline $\begin{array}{l}\text { 82. Pourcentage de clientes PF qui } \\
\text { connaissent bien leur méthode }\end{array}$ & $\checkmark$ & & & & \\
\hline & & & DIU : 82-87 & $\begin{array}{l}\text { F82 } \\
\text { F83X1 à F83X98, } \\
\text { F84 } \\
\text { F85X1 à F85X98, } \\
\text { F86,F87 }\end{array}$ & \\
\hline & & & inj : 92-95 & $\begin{array}{l}\text { F92, } \\
\text { F93X1 à F93X98 } \\
\text { F94X1 à F94X98 } \\
\text { F95 }\end{array}$ & \\
\hline $\begin{array}{l}\text { 83. Pourcentage de clientes PF qui } \\
\text { connaissent bien les } \\
\text { symptômes des MST/NIH }\end{array}$ & & $\mathrm{F}$ & 118,119 & $\begin{array}{l}\text { F118 } \\
\text { F119X1 à } \\
\quad \text { F119X98 }\end{array}$ & $\begin{array}{l}\text { Ces indicateurs peuvent être rapportés } \\
\text { comme un groupe d'éléments que les } \\
\text { clientes connaissent, ou bien ils peuvent } \\
\text { être combinés en scores de } \\
\text { connaissance. }{ }^{4}\end{array}$ \\
\hline $\begin{array}{l}\text { 84. Pourcentage de clientes PF qui } \\
\text { connaissent bien les voies de } \\
\text { transmission du VIH } \\
\end{array}$ & & $\mathrm{F}$ & 120,121 & $\begin{array}{l}\mathrm{F} 120 \\
\mathrm{~F} 121 \mathrm{X} 1 \mathrm{à} \\
\mathrm{F} 121 \times 98 \\
\end{array}$ & $\begin{array}{l}\text { Ces résultats peuvent être comparés aussi } \\
\text { à ceux des clientes SMI (interview SMI } \\
\text { q.66-73). }\end{array}$ \\
\hline $\begin{array}{l}\text { 85. Pourcentage de clientes PF qui } \\
\text { connaissent bien la protection } \\
\text { contre les MST/NIH } \\
\end{array}$ & $\checkmark$ & $\mathrm{F}$ & 122,123 & $\begin{array}{l}\text { F122 } \\
\text { F123X1 à } \\
\text { F123X98 }\end{array}$ & \\
\hline \multirow{3}{*}{$\begin{array}{l}\text { 86. Pourcentage de clientes PF qui } \\
\text { connaissent d'autres services } \\
\text { offerts dans ce PPS }\end{array}$} & & $\mathrm{F}$ & 30 & F30X1 à F30X98 & \multirow{3}{*}{$\begin{array}{l}\text { Si l'interview de sortie de la cliente PF est } \\
\text { liée à l'inventaire, }{ }^{3} \text { alors vous pouvez } \\
\text { comparer les services qui sont } \\
\text { disponibles avec ceux que les clientes } \\
\text { connaissent (voir l'indicateur 12). Vous } \\
\text { pouvez trouver que les clientes ne sont } \\
\text { pas au courant des services disponibles, } \\
\text { ou bien elles peuvent avoir l'impression } \\
\text { que certains services sont disponibles } \\
\text { alors qu'ils ne le sont pas. } \\
\text { Ces résultats peuvent être comparés à } \\
\text { ceux des clientes SMI (interview SMI } \\
\text { q.31). }\end{array}$} \\
\hline & & 1 & 8 & $18 \mathrm{~A} 1$ à $18 \mathrm{~A} 17$ & \\
\hline & & & & & \\
\hline
\end{tabular}

$I=$ Inventaire 0 = Guide d'observation $F=$ Interview de sortie de la cliente PF $S=$ Interview du personnel $M=$ Interview de sortie de la cliente de SMI 
Plan d'analyse : Qualité des soins

\begin{tabular}{|c|c|c|c|c|c|}
\hline \multicolumn{2}{|l|}{$\begin{array}{l}\text { Indicateur de la qualité } \\
\checkmark=\text { Indicateur fréquemment utilisé }\end{array}$} & \multirow{2}{*}{$\begin{array}{l}\text { Instr } \\
\text { F }\end{array}$} & \multirow{2}{*}{$\begin{array}{l}\text { Numéros des } \\
\text { questions } \\
62,63\end{array}$} & \multirow{2}{*}{$\begin{array}{l}\text { Variables } \\
\text { F62, F63 }\end{array}$} & \multirow{2}{*}{$\begin{array}{l}\text { Commentaires } \\
\text { Cet indicateur traite du lien entre la } \\
\text { continuité de l'utilisation et le fait de } \\
\text { recevoir l'information sur les effets } \\
\text { secondaires. Les résultats peuvent révéler } \\
\text { que celles qui veulent changer ou arrêter à } \\
\text { cause d'effets secondaires peuvent avoir } \\
\text { ne pas été bien informées de ces effets } \\
\text { depuis le départ. Cependant, les résultats } \\
\text { ne sont que suggestifs, puisque les } \\
\text { clientes qui arrêtent ne reviennent pas le } \\
\text { plus souvent dans le PPS, de ce fait elles } \\
\text { ne sont pas incluses dans l'indicateur. }\end{array}$} \\
\hline $\begin{array}{l}\text { 87. Parmi les anciennes clientes PF } \\
\text { qui ont connu des effets } \\
\text { secondaires avec leurs } \\
\text { méthodes, pourcentage de } \\
\text { celles qui avaient été informées } \\
\text { des effets secondaires } \\
\text { Iorsqu'elles ont reçu la méthode } \\
\text { pour la première fois }\end{array}$ & & & & & \\
\hline $\begin{array}{l}\text { 88. Pourcentage de clientes SMI } \\
\text { qui connaissent les méthodes } \\
\text { disponibles dans ce PPS }\end{array}$ & & M & 41 & M41X1 à M41X13 & $\begin{array}{l}\text { Si l'interview de sortie de la cliente PF est } \\
\text { liée à l'inventaire,3 alors vous pouvez } \\
\text { comparer les méthodes qui sont } \\
\text { réellement disponibles avec celles que les } \\
\text { clientes SMI connaissent (voir l'indicateur } \\
\text { 11). }\end{array}$ \\
\hline $\begin{array}{l}\text { 89. Pourcentage de clientes SMI } \\
\text { qui ont vu une information } \\
\text { relative à la PF pendant la visite }\end{array}$ & & M & 42,43 & $\begin{array}{l}\text { M42, } \\
\text { M43X1 à M43X5 }\end{array}$ & \\
\hline \multicolumn{6}{|l|}{ Compétence technique } \\
\hline $\begin{array}{l}\text { 90. Pourcentage de clientes PF } \\
\text { examinées cliniquement pour la } \\
\text { PF }\end{array}$ & $\checkmark$ & 0 & 3,4 & $\begin{array}{l}03 \times 1,03 \times 2 \\
04 \times 1 \text { à } 04 \times 7\end{array}$ & $\begin{array}{l}\text { Les politiques des programmes de PF } \\
\text { spécifient souvent les examens qui } \\
\text { devraient être faits chez les clientes. } \\
\text { Référez-vous à ces standard en } \\
\text { sélectionnant les éléments à inclure dans } \\
\text { cet indicateur. } \\
\text { Présentez sous forme de diagramme en } \\
\text { bâtons. Ceci est généralement rapporté } \\
\text { séparément pour les nouvelles clientes et } \\
\text { pour les anciennes. }\end{array}$ \\
\hline $\begin{array}{l}\text { 91. Pourcentage d'examens } \\
\text { pelviens pendant lesquels les } \\
\text { prestataires ont fait diverses } \\
\text { action }\end{array}$ & $\checkmark$ & 0 & 37,38 & $\begin{array}{l}037 X 1 \text { à } 037 X 77 \\
038 X 1 \text { à } 038 X 77\end{array}$ & $\begin{array}{l}\text { Présentez sous forme de diagramme en } \\
\text { bâtons pour les } 9 \text { actions énumérées. } \\
\text { Notez que l'unité d'analyse est l'examen } \\
\text { pelvien, et non pas le prestataire ou la } \\
\text { cliente. }\end{array}$ \\
\hline $\begin{array}{l}\text { 92. Pourcentage de poses de DIU } \\
\text { pendant lesquelles les } \\
\text { prestataires ont fait diverses } \\
\text { actions }\end{array}$ & $\checkmark$ & 0 & 39 & 039X1 à 039X77 & $\begin{array}{l}\text { Présentez sous forme de diagramme en } \\
\text { bâtons pour les } 4 \text { actions énumérées. } \\
\text { Notez que l'unité d'analyse est la pose du } \\
\text { DIU, et non pas le prestataire ou la cliente }\end{array}$ \\
\hline
\end{tabular}


Plan d'analyse : Qualité des soins

\begin{tabular}{|c|c|c|c|c|c|}
\hline $\begin{array}{l}\text { Indicateur de la qualité } \\
\checkmark=\text { Indicateur fréquemment utilisé }\end{array}$ & & Instr & $\begin{array}{l}\text { Numéros des } \\
\text { questions }\end{array}$ & Variables & Commentaires \\
\hline $\begin{array}{l}\text { 93. Pourcentage d'injections } \\
\text { pendant lesquelles les } \\
\text { prestataires ont fait diverses } \\
\text { actions } \\
\end{array}$ & $\checkmark$ & 0 & 40 & $040 \times 1$ à 040X77 & $\begin{array}{l}\text { Présentez sous forme de diagramme en } \\
\text { bâtons pour les } 3 \text { actions énumérées. } \\
\text { Notez que l'unité d'analyse est l'injection, } \\
\text { et non pas le prestataire ou la cliente. }\end{array}$ \\
\hline $\begin{array}{l}\text { 94. Pourcentage de poses de } \\
\text { NORPLANT }{ }^{\circledR} \text { pendant } \\
\text { lesquelles les prestataires ont } \\
\text { fait diverses actions }\end{array}$ & $\checkmark$ & 0 & 41 & $041 \times 1$ à 041X77 & $\begin{array}{l}\text { Présentez sous forme de diagramme en } \\
\text { bâtons pour les } 4 \text { actions énumérées. } \\
\text { Notez que l'unité d'analyse est la pose du } \\
\text { NORPLANT }^{\circledast} \text {, et non pas le prestataire ou } \\
\text { la cliente. }\end{array}$ \\
\hline $\begin{array}{l}\text { 95. Pourcentage de procédures } \\
\text { pendant lesquelles les } \\
\text { prestataires ont utilisé des } \\
\text { gants stériles }\end{array}$ & $\checkmark$ & 0 & 42 & 042 & $\begin{array}{l}\text { Ceci est souvent rapporté seulement pour } \\
\text { les poses de DIU et de NORPLANT } \\
\text { puisqu'une stérilité stricte n'est pas } \\
\text { totalement nécessaire pour les autres } \\
\text { procédures. }\end{array}$ \\
\hline $\begin{array}{l}\text { 96. Pourcentage de prestataires } \\
\text { observés entreprenant une } \\
\text { action avec les clientes PF qui } \\
\text { ont des problèmes avec leur } \\
\text { méthode }\end{array}$ & $\checkmark$ & 0 & 8,9 & $\begin{array}{l}08 \\
09 \times 1 \text { à } 09 \times 77\end{array}$ & $\begin{array}{l}\text { Présentez sous forme de diagramme en } \\
\text { camembert. Utilisez la q. } 8 \text { comme filtre. }\end{array}$ \\
\hline $\begin{array}{l}\text { 97. Pourcentage d'anciennes } \\
\text { clientes PF ayant des } \\
\text { problèmes et qui ont le } \\
\text { sentiment qu'elles ont reçu une } \\
\text { attention particulière de la part } \\
\text { du prestataire } \\
\end{array}$ & & $\mathrm{F}$ & 65 & F65 & Comparez ceci à l'indicateur précédent \\
\hline $\begin{array}{l}\text { 98. Pourcentage de prestataires qui } \\
\text { rapportent qu'ils traiteraient } \\
\text { correctement une cliente sous } \\
\text { pilule même si elle n'a pas ses } \\
\text { règles au moment de la } \\
\text { consultation } \\
\end{array}$ & & S & 23 & S23X1 à S23X7 & $\begin{array}{l}\text { Présentez sous forme de diagramme en } \\
\text { camembert. }\end{array}$ \\
\hline $\begin{array}{l}\text { 99. Pourcentage de clientes PF } \\
\text { examinées correctement pour } \\
\text { les MST }\end{array}$ & $\checkmark$ & 0 & 3,4 & $\begin{array}{l}03 \times 3 \text { à } 03 \times 6 \\
04 \times 8\end{array}$ & \\
\hline $\begin{array}{l}\text { 100. Pourcentage de prestataires } \\
\text { observés entreprenant une } \\
\text { action avec des clientes } \\
\text { soupçonnées avoir une MST }\end{array}$ & $\checkmark$ & 0 & 18,19 & $\begin{array}{l}018 \\
019 \times 1 \text { à } 019 \times 77\end{array}$ & $\begin{array}{l}\text { Présentez sous forme de diagramme en } \\
\text { bâtons. Utilisez la q. } 18 \text { comme filtre. }\end{array}$ \\
\hline $\begin{array}{l}\text { 101. Pourcentage de prestataires } \\
\text { rapportant qu'ils } \\
\text { entreprendraient une action } \\
\text { avec des clientes ayant des } \\
\text { MST }\end{array}$ & & S & 26 & S26X1 à S26X9 & $\begin{array}{l}\text { Présentez sous forme de diagramme en } \\
\text { bâtons. Comparez ceci à l'indicateur } \\
\text { précédent. }\end{array}$ \\
\hline
\end{tabular}

$I=$ Inventaire $\mathrm{O}=$ Guide d'observation $\mathrm{F}=$ Interview de sortie de la cliente PF $\mathrm{S}=$ Interview du personnel $\mathrm{M}=$ Interview de sortie de la cliente de SMI 
Plan d'analyse : Qualité des soins

\begin{tabular}{|c|c|c|c|c|c|}
\hline \multicolumn{2}{|l|}{$\begin{array}{l}\text { Indicateur de la qualité } \\
\checkmark=\text { Indicateur fréquemment utilisé }\end{array}$} & \multirow{2}{*}{\begin{tabular}{|l} 
Instr \\
S
\end{tabular}} & \multirow{2}{*}{$\begin{array}{l}\text { Numéros des } \\
\text { questions } \\
27\end{array}$} & \multirow{2}{*}{\begin{tabular}{|l|} 
Variables \\
S27X1 à S27X8 \\
\end{tabular}} & \multirow{2}{*}{\begin{tabular}{|l} 
Commentaires \\
$\begin{array}{l}\text { Présentez sous forme de diagramme en } \\
\text { bâtons. }\end{array}$
\end{tabular}} \\
\hline $\begin{array}{l}\text { 102. Pourcentage de prestataires } \\
\text { rapportant qu'ils } \\
\text { entreprendraient une action } \\
\text { avec des clientes ayant le } \\
\text { VIH/SIDA } \\
\end{array}$ & & & & & \\
\hline $\begin{array}{l}\text { 103. Pourcentage de prestataires } \\
\text { rapportant qu'ils testent pour le } \\
\text { syphilis les clientes prénatales }\end{array}$ & & S & 28 & $\mathrm{~S} 28$ & \\
\hline $\begin{array}{l}\text { 104. Pourcentage de prestataires qui } \\
\text { rapportent qu'ils ne } \\
\text { recommanderaient pas de } \\
\text { méthodes aux clientes ayant } \\
\text { des MST }\end{array}$ & & S & $21,21 a$ & $\begin{array}{l}\text { S21 } \\
\text { S21AX1 à } \\
\text { S21AX77 }\end{array}$ & $\begin{array}{l}\text { Présentez sous forme de diagramme en } \\
\text { bâtons, par méthode. }\end{array}$ \\
\hline $\begin{array}{l}105 \text { Pourcentage de prestataires qui } \\
\text { rapportent divers conseils qu'ils } \\
\text { donneraient à une utilisatrice de } \\
\text { la pilule qui coure un risque } \\
\text { élevé de MST } \\
\end{array}$ & & S & 25 & S25X1 à S25X98 & $\begin{array}{l}\text { Présentez sous forme de diagramme en } \\
\text { bâtons, par méthode }\end{array}$ \\
\hline $\begin{array}{l}\text { 106. Pourcentage de clientes PF qui } \\
\text { reçoivent d'autres services }\end{array}$ & $\checkmark$ & $\mathrm{F}$ & 3,4 & $\begin{array}{l}F 3, \\
F 4 X 1 \text { à F4X16 }\end{array}$ & $\begin{array}{l}\text { Présentez sous forme de diagramme en } \\
\text { bâtons. } \\
\text { Ces résultats peuvent être comparés à } \\
\text { ceux des clientes SMI (interview SMI q.3). }\end{array}$ \\
\hline \multicolumn{6}{|c|}{ Mécanismes pour encourager la continuité } \\
\hline $\begin{array}{l}\text { 107. Pourcentage de clientes PF } \\
\text { observées avoir reçu la date de } \\
\text { leur visite de suivi par écrit }\end{array}$ & & 0 & 33,34 & 033,034 & $\begin{array}{l}\text { Présentez sous forme de diagramme en } \\
\text { camembert avec trois tranches : pas de } \\
\text { date de suivi, date de suivi non écrite et } \\
\text { date de suivi écrite. }\end{array}$ \\
\hline $\begin{array}{l}\text { 108. Pourcentage de clientes PF } \\
\text { rapportant avoir reçu une date } \\
\text { de visite de suivi }\end{array}$ & $\checkmark$ & $\mathrm{F}$ & 22 & F22 & $\begin{array}{l}\text { Pour comparer celui-ci à l'indicateur } \\
\text { précédent, liez l'interview de sortie de la } \\
\text { cliente PF avec l'observation. }{ }^{3}\end{array}$ \\
\hline $\begin{array}{l}\text { 109. Pourcentage de clientes PF qui } \\
\text { ont reçu une provision de leur } \\
\text { méthode aujourd'hui }\end{array}$ & $\checkmark$ & 0 & 28 & 028 & \\
\hline $\begin{array}{l}\text { 110. Moyenne des cycles de pilules } \\
\text { fournies aux clientes PF }\end{array}$ & & 0 & $6,23,25,32$ & $\begin{array}{l}06,023,025 \\
032\end{array}$ & $\begin{array}{l}\text { D'abord, déterminez la méthode utilisée, à } \\
\text { partir des q.6,23 et } 25 \text {, en fonction du } \\
\text { type de cliente. Ensuite donnez la quantité } \\
\text { fournie, à partir de la q.32. }\end{array}$ \\
\hline $\begin{array}{l}\text { 111. Pourcentage de clientes sous } \\
\text { pilule rapportant avoir reçu une } \\
\text { provision de pilules }\end{array}$ & & $\mathrm{F}$ & 78 & F78 & $\begin{array}{l}\text { Afin de comparer cela à l'observation, liez } \\
\text { les dossiers d'observation et d'interview } \\
\text { de sortie. }\end{array}$ \\
\hline
\end{tabular}

$\mathrm{I}=$ Inventaire $\mathrm{O}=$ Guide d'observation $\mathrm{F}=$ Interview de sortie de la cliente PF $\mathrm{S}=$ Interview du personnel $\mathrm{M}=$ Interview de sortie de la cliente de SMI 
Plan d'analyse : Qualité des soins

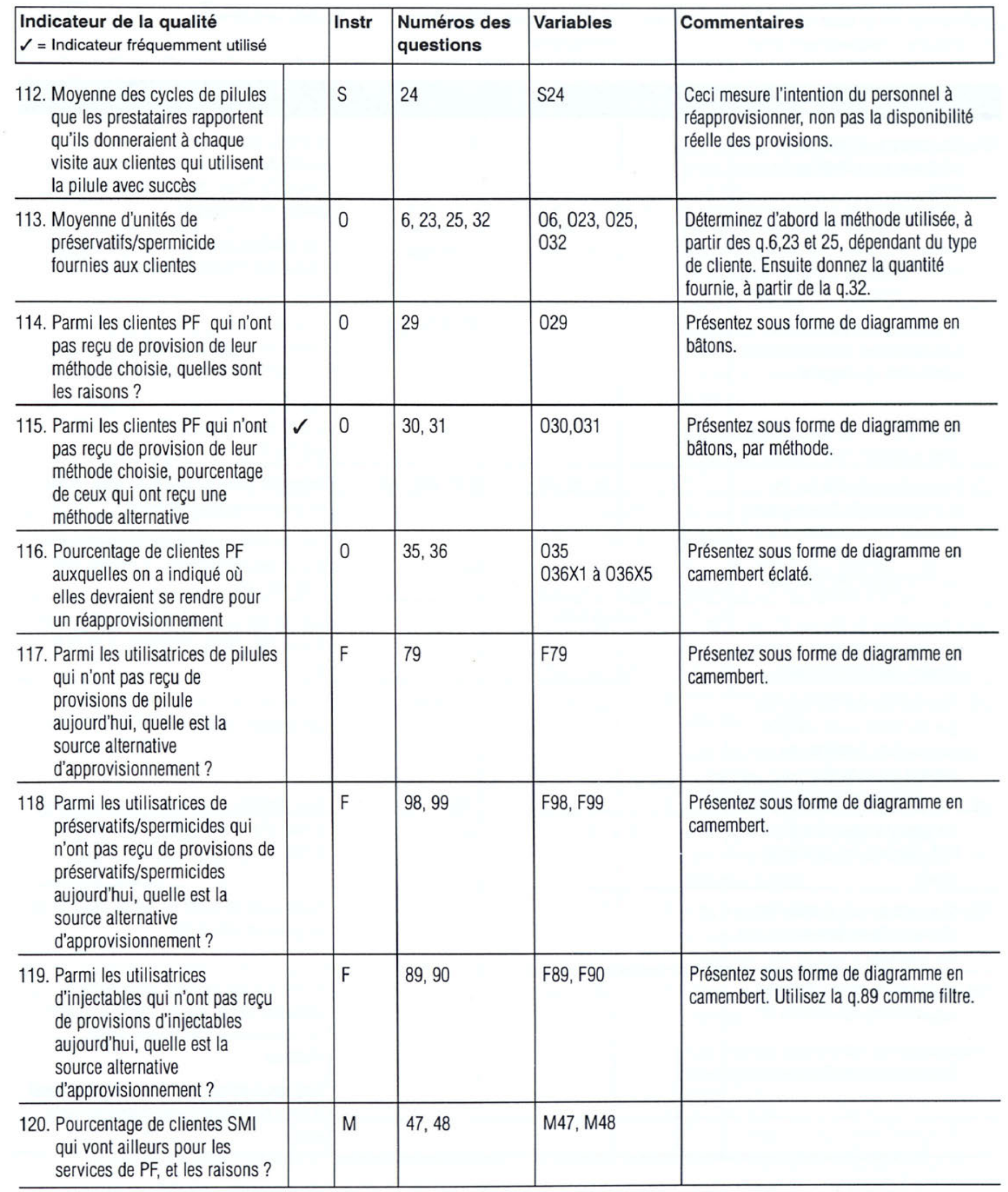


Plan d'analyse : Qualité des soins

\begin{tabular}{|c|c|c|c|c|c|}
\hline $\begin{array}{l}\text { Indicateur de la qualité } \\
\checkmark=\text { Indicateur fréquemment utilisé }\end{array}$ & & Instr & $\begin{array}{l}\text { Numéros des } \\
\text { questions }\end{array}$ & Variables & Commentaires \\
\hline Caractère approprié et acceptabilit & do & servic & & & \\
\hline $\begin{array}{l}\text { 121. Pourcentage de clientes PF } \\
\text { généralement satisfaites de leur } \\
\text { visite }\end{array}$ & & $\mathrm{F}$ & 1 & $\mathrm{~F} 1$ & $\begin{array}{l}\text { Souvent, cet indicateur a un résultat très } \\
\text { positif parce que les clientes veulent être } \\
\text { polies. Ce "biais de courtoisie" réduit la } \\
\text { validité de l'indicateur. } \\
\text { Ces résultats peuvent être comparés aussi } \\
\text { à ceux des clientes SMI (interview SMI } \\
\text { q.2). }\end{array}$ \\
\hline $\begin{array}{l}\text { 122. Pourcentage de clientes PF } \\
\text { satisfaites avec certains aspects } \\
\text { particuliers des services }\end{array}$ & $\checkmark$ & $\mathrm{F}$ & $5,15,16$ & $\mathrm{~F} 5, \mathrm{~F} 15, \mathrm{~F} 16$ & $\begin{array}{l}\text { Ceux-ci incluent : la cliente a reçu } \\
\text { l'information qu'elle voulait, a eu assez } \\
\text { d'intimité, et a compris le prestataire. } \\
\text { Ces résultats peuvent être comparés aussi } \\
\text { à ceux des clientes SMI (interview SMI } \\
\text { q.4,14,15). }\end{array}$ \\
\hline $\begin{array}{l}\text { 123. Pourcentage de clientes PF } \\
\text { ayant d'autres PPS plus proche } \\
\text { de leurs maisons, et la raison } \\
\text { de venir dans ce PPS au lieu } \\
\text { du plus proche de chez elles }\end{array}$ & & $\mathrm{F}$ & $31,32,33$ & F31, F32, F33 & $\begin{array}{l}\text { Présentez spécialement q.33 sous forme } \\
\text { de diagramme en camembert. } \\
\text { Ces résultats peuvent être comparés aussi } \\
\text { à ceux des clientes SMI (interview SMI } \\
\text { q.32, 33, 34). }\end{array}$ \\
\hline $\begin{array}{l}\text { 124. Pourcentage de clientes PF qui } \\
\text { trouvent que les horaires de la } \\
\text { clinique sont convenables }\end{array}$ & $\checkmark$ & $\mathrm{F}$ & 23 & F23 & $\begin{array}{l}\text { Ces résultats peuvent être comparés aussi } \\
\text { à ceux des clientes SMI (interview SMI } \\
\text { q.27). }\end{array}$ \\
\hline $\begin{array}{l}\text { 125. Pour les clientes qui trouvent } \\
\text { que les horaires ne sont pas } \\
\text { convenables, horaires qui } \\
\text { seraient convenables }\end{array}$ & & $\mathrm{F}$ & 23,24 & $\mathrm{~F} 23, \mathrm{~F} 24$ & $\begin{array}{l}\text { Présentez sous forme de diagramme en } \\
\text { camembert. Utilisez la q. } 23 \text { comme filtre. }\end{array}$ \\
\hline $\begin{array}{l}\text { 126. Pourcentage de clientes PF qui } \\
\text { ont déjà été renvoyées de ce } \\
\text { PPS pendant des heures de } \\
\text { travail }\end{array}$ & & $\mathrm{F}$ & 25 & $\mathrm{~F} 25$ & $\begin{array}{l}\text { Ces résultats peuvent être comparés aussi } \\
\text { à ceux des clientes SMI (interview SMI } \\
\text { q.28). }\end{array}$ \\
\hline $\begin{array}{l}\text { 127. Moyenne du retard entre l'heure } \\
\text { officielle d'ouverture et le début } \\
\text { des services }\end{array}$ & $\checkmark$ & 1 & $1,2,3$ & & $\begin{array}{l}\text { Notez aussi la durée du temps d'attente de } \\
\text { la première cliente PF. }\end{array}$ \\
\hline $\begin{array}{l}\text { 128. Moyenne du temps d'attente } \\
\text { rapporté chez les clientes PF }\end{array}$ & $\checkmark$ & $\mathrm{F}$ & 26 & F26 & $\begin{array}{l}\text { Notez que ces données sont souvent } \\
\text { inexactes parce que les clientes peuvent } \\
\text { ne pas prendre note de leur temps } \\
\text { d'attente. } \\
\text { Ces résultats peuvent être comparés aussi } \\
\text { à ceux des clientes SMI (interview SMI } \\
\text { q.16). }\end{array}$ \\
\hline
\end{tabular}

$\mathrm{I}=$ Inventaire $\mathrm{O}=$ Guide d'observation $\mathrm{F}=$ Interview de sortie de la cliente PF $\mathrm{S}=$ Interview du personnel $\mathrm{M}=$ Interview de sortie de la cliente de SMI 
Plan d'analyse : Qualité des soins

\begin{tabular}{|c|c|c|c|c|c|}
\hline $\begin{array}{l}\text { Indicateur de la qualité } \\
\checkmark=\text { Indicateur fréquemment utilisé }\end{array}$ & & Instr & $\begin{array}{l}\text { Numéros des } \\
\text { questions }\end{array}$ & Variables & Commentaires \\
\hline $\begin{array}{l}\text { 129. Pourcentage de clientes } \\
\text { satisfaites du temps d'attente }\end{array}$ & $\checkmark$ & $F$ & 26,27 & F26, F27 & $\begin{array}{l}\text { Comparez ceci au temps d'attente rapporté } \\
\text { ci-dessus. } \\
\text { Ces résultats peuvent être comparés aussi } \\
\text { à ceux des clientes SMI (interview SMI } \\
\text { q.16,17). }\end{array}$ \\
\hline $\begin{array}{l}\text { 130. Durée moyenne, minimale, } \\
\text { maximale de la consultation de } \\
\text { PF }\end{array}$ & $\checkmark$ & 0 & $\begin{array}{l}\text { Page } 53 \\
\text { page } 59\end{array}$ & $\begin{array}{l}\text { DEBUT } \\
\text { FIN, DUREE }\end{array}$ & $\begin{array}{l}\text { Ceci est généralement rapporté } \\
\text { séparément pour les nouvelles clientes et } \\
\text { les anciennes clientes. Utiliser le DEBUT } \\
\text { et la FIN pour vérifier la DUREE. }\end{array}$ \\
\hline $\begin{array}{l}\text { 131. Pourcentage de clientes } \\
\text { satisfaites de la durée de la } \\
\text { consultation de PF }\end{array}$ & $\checkmark$ & $F$ & 6 & F6 & $\begin{array}{l}\text { Comparez ceci au temps de consultation } \\
\text { observé, ci-dessus. Ceci implique la } \\
\text { liaison de l'observation avec l'interview de } \\
\text { sortie. } 3 \\
\text { Ces résultats peuvent être comparés aussi } \\
\text { à ceux des clientes SMI (interview SMI } \\
\text { q.5). }\end{array}$ \\
\hline $\begin{array}{l}\text { 132. Coât moyen demandé pour } \\
\text { chaque méthode }\end{array}$ & $\checkmark$ & 1 & 38 & $\begin{array}{l}\text { I38A1 à I38A12 } \\
\text { I38B1 à I38B12 }\end{array}$ & $\begin{array}{l}\text { Ils sont généralement rapportés par } \\
\text { secteuir. }\end{array}$ \\
\hline $\begin{array}{l}\text { 133. Coût moyen demandé pour les } \\
\text { services de MST/NIH, } \\
\text { prénatals, postnatals, SR et SMI }\end{array}$ & $\checkmark$ & 1 & 38 & $\begin{array}{l}\text { I38A13 à I38A26 } \\
\text { I38B13 à I38B26 }\end{array}$ & $\begin{array}{l}\text { encourus par les clientes (indicateurs } 134 \\
\text { et 135). }\end{array}$ \\
\hline $\begin{array}{l}\text { 134. Coût moyen payé par les } \\
\text { clientes pour la } \\
\text { consultation/médicaments/autre } \\
\text { s consommables }\end{array}$ & & $F$ & $34,35,36$ & F34, F35, F36 & $\begin{array}{l}\text { On peut rapporter cet indicateur combiné } \\
\text { avec l'indicateur suivant comme le total du } \\
\text { co0̂t aux clientes. } \\
\text { Ces résultats peuvent être comparés aussi } \\
\text { à ceux des clientes SMI (variables de } \\
\text { l'interview SMI M37X1 à M37X3). } \\
\end{array}$ \\
\hline $\begin{array}{l}\text { 135. Cout moyen payé par les } \\
\text { clientes pour l'inscription, le } \\
\text { voyage, et autre }\end{array}$ & & $\mathrm{F}$ & $37,38,39$ & F37, F38, F39 & $\begin{array}{l}\text { Peut rapporter cet indicateur combiné } \\
\text { avec l'indicateur suivant comme le total du } \\
\text { coat aux clientes. } \\
\text { Ces résultats peuvent être comparés aussi } \\
\text { à ceux des clientes SMI (variables de } \\
\text { l'interview SMI M37X4 à M37X6). } \\
\end{array}$ \\
\hline $\begin{array}{l}\text { 136. Pourcentage de clientes PF } \\
\text { satisfaites du coat de leurs } \\
\text { services }\end{array}$ & & $F$ & 40 & F40 & $\begin{array}{l}\text { Comparez ceci au coQt payé pour les } \\
\text { services. } \\
\text { Ces résultats peuvent être comparés aussi } \\
\text { à ceux des clientes SMI (interview SMI } \\
\text { q.38). }\end{array}$ \\
\hline
\end{tabular}

I = Inventaire $\mathrm{O}=$ Guide d'observation $\mathrm{F}=$ Interview de sortie de la cliente PF $\mathrm{S}=$ Interview du personnel $\mathrm{M}=$ Interview de sortie de la cliente de SMI 


\section{Plan d'analyse : Qualité des soins}

\begin{tabular}{|l|l|l|l|l|l|}
\hline \multicolumn{2}{|l|}{$\begin{array}{l}\text { Indicateur de la qualité } \\
\checkmark=\text { Indicateur fréquemment utilisé }\end{array}$} & Instr & $\begin{array}{l}\text { Numéros des } \\
\text { questions }\end{array}$ & Variables & Commentaires \\
$\begin{array}{c}\text { 137. Moyenne du temps de voyage } \\
\text { nécessaire pour les clientes PF }\end{array}$ & $\checkmark$ & $F$ & 28 & F28 & $\begin{array}{l}\text { Ces résultats peuvent être comparés aussi } \\
\text { à ceux des clientes SMI (interview SMI } \\
\text { q.29). }\end{array}$ \\
\hline $\begin{array}{c}\text { 138. Pourcentage de clientes PF } \\
\text { utilisant divers moyens de } \\
\text { transport pour se rendre au } \\
\text { PPS }\end{array}$ & & F & 29 & F29 & $\begin{array}{l}\text { Présentez sous forme de diagramme en } \\
\text { camembert. } \\
\text { Ces résultats peuvent être comparés aussi } \\
\text { à ceux des clientes SMI (interview SMI } \\
\text { q.30). }\end{array}$ \\
\hline
\end{tabular}

$I=$ Inventaire 0 = Guide d'observation $F=$ Interview de sortie de la cliente PF $S=$ Interview du personnel $M=$ Interview de sortie de la cliente de SMI 


\section{Notes sur le plan d'analyse}

1. Cette analyse est généralement faite de la manière suivante : D'abord, calculez le taux de la clientèle PF pour tous les PPS, comme le demande l'indicateur 13. Ensuite, triez les PPS selon le taux de fréquentation de la clientèle, avec le taux de fréquentation le plus élevé en premier, en progressant vers les taux les plus faibles. Avec les PPS dans cet ordre, répartissez-les en quatre groupes égaux ou quartiles. S'il y a 200 PPS dans l'étude, ceci donnera quatre groupes de 50 PPS, le premier groupe étant constitué des 50 PPS les plus grands, et le quatrième groupe constitué des 50 PPS les plus petits.

Chacun de ces quatre groupes sert un certain pourcentage du taux de fréquentation total de la clientèle. A partir de l'information sur le taux de fréquentation de la clientèle, il est possible de calculer le nombre total de clientes servies par l'ensemble du système de planification familiale. Ce total peut être utilisé comme le dénominateur pour déterminer le pourcentage de clientes servies par chaque quartile de PPS.

Par exemple, admettons que les 200 PPS mentionnés ci-dessus traitent 12.000 visites de clientes par an. Cela donne une moyenne de 60 clientes par PPS et par an. Mais certains PPS sont assez grands et d'autres assez petits. Si les plus grands quartiles sont réunis ensemble, nous pouvons trouver qu'en tant que groupe, ils servent 8.000 clientes, ou $75 \%$ du total du volume annuel de la clientèle. Le reste des quartiles peuvent servir $12 \%, 8 \%$, et $5 \%$ du volume total de la clientèle, respectivement. Ces résultats très biaisés sont typiques, et peuvent révéler des différences dans la qualité entre les PPS de forte fréquentation et ceux de faible fréquentation.

2. Les indicateurs sur les barrières aux services portent sur quatre barrières (l'âge, le statut matrimonial, la parité et le consentement de l'époux), et sur cinq méthodes ( $\mathrm{COC}$, préservatif, DIU, injectables et stérilisation). L'idée des indicateurs 61 et 62 est de déterminer la proportion des prestataires qui imposent ces barrières, aussi bien que l'ampleur de ces barrières pour ceux qui les imposent.

L'indicateur 61 nécessite une estimation des prestataires qui imposent chaque barrière sur chaque méthode. Par exemple, combien de prestataires exigent qu'une cliente soit mariée avant de lui prescrire le DIU ? Chaque combinaison de barrière et de méthode aboutira à un résultat spécifique, donnant 20 éléments d'information (4 barrières X 5 méthodes). Cette information est généralement présentée sous forme de de tableau ou de diagramme en bâtons, par méthode.

Le pourcentage de prestataires qui imposent les barrières de parité, du statut matrimonial et du consentement de l'époux sur chaque méthode peut être calculé facilement à partir des questions 15,17 et 18 de l'interview du personnel. Le pourcentage de prestataires qui imposent la barrière de l'âge est plus compliqué. Dans les questions 11 et 12 , on demande d'abord aux personnels s'il y a un âge minimum au dessous duquel ils ne prescriront pas les méthodes, et si c'est le cas, on leur demande de spécifier cet âge. Par exemple, si un prestataire dit qu'il n'a pas d'exigences d'âge minimum pour les pilules, alors il est clair qu'il n'impose pas de barrière d'âge. Si un prestataire dit qu'il exige que la cliente ait 21 ans avant de lui fournir la pilule, alors il est clair qu'il impose une barrière d'âge.

Mais certains prestataires peuvent dire qu'ils exigent que la cliente ait seulement 10 ou 12 ans pour lui fournir la pilule. Selon les standard de nombreux programmes, ceci ne constitue pas effectivement une barrière d'âge. De même, certains prestataires peuvent dire que leur âge maximum limite est de 75 ans pour la distribution des préservatifs. Il est clair qu'il ne s'agit là que d'une barrière d'âge qui n'existe que de nom, et ne pose pas une véritable barrière pour les clientes. Dans ces cas, les prestataires qui déclarent qu'ils ont des barrières d'âge minimum ou maximum mais dont les seuils sont extrêmement bas ou hauts, ne devraient pas être considérés comme imposant des barrières d'âge. L'analyste doit s'entendre avec les responsables des programmes 
sur les âges minimum et maximum qui seront considérés comme de vraies barrières pour chaque méthode. Ainsi, les prestataires dont les contraintes d'âge sont en dehors de ces seuils ne seront pas considérés comme imposant des barrières d'âge. Les données des questions 11 et 14 doivent être analysées attentivement avec ces éléments en tête.

L'indicateur 62 lui-même traite de la nature des barrières d'âge et de parité qui sont imposées. Autrement dit, parmi les prestataires qui imposent un âge minimum limite pour les pilules, quel est l'âge moyen exigé ? Ces moyennes ne devraient être calculées que chez les prestataires qui imposent en réalité une vraie barrière d'âge. Rappelez-vous que les prestataires dont les barrières d'âge sont hors de ces limites fixées pour l'indicateur 61 ne devraient pas être inclus dans ces moyennes. Quant à la parité, l'exigence même d'un enfant pour n'importe quelle méthode (y compris le DIU) est généralement considérée comme une barrière, de ce fait cette moyenne devrait être calculée pour tous les prestataires qui déclarent dans la question 15 qu'ils imposent toute sorte d'exigence de parité quelle que soit cette parité.

3. Le fait de lier les données entre les modules nécessite une patience, mais il permet une analyse beaucoup plus riche et plus informative. Pour plusieurs indicateurs, les liaisons sont nécessaires entre l'inventaire et l'observation; entre l'inventaire et l'interview de sortie de la cliente PF; et entre l'observation et l'interview de sortie de la cliente PF. En d'autres termes, il est souvent nécessaire de savoir non seulement dans quel PPS une observation particulière a eu lieu, mais aussi d'avoir accès à l'information relative à l'inventaire de ce PPS. De même, il est souvent nécessaire d'avoir l'information à partir de l'observation d'une cliente particulière pour faire la comparaison avec ses réponses pendant l'interview de sortie.

Ceci se fait à travers les variables d'identification qui se trouvent sur la première page de chaque questionnaire. Par exemple, l'indicateur 76 est "Pourcentage de clientes PF avec lesquelles des matériels d'IEC ont été utilisés (dans les PPS qui disposent de matériels d'IEC)." Ceci nécessite l'établissement d'une liaison entre l'inventaire-pour savoir si les matériels d'IEC étaient présents-et l'observation-pour savoir si les matériels ont été réellement utilisés pendant la consultation. L'inventaire et l'observation ont tous les deux la variable CODEFS, qui devrait identifier la formation sanitaire dans les deux modules. Dans l'observation, la variable CODEFS est la liaison qui renvoie à l'inventaire qui, à son tour, fournit l'information sur la disponibilité de l'IEC. De même, l'observation et l'interview de sortie partagent une variable IDCL, qui devrait être la même pour toutes les clientes. Cette variable devrait lier l'observation et l'interview de sortie directement.

Ces variables de liaison ne doivent identifier que des cas uniques. C'est-à-dire que la variable CODEFS doit avoir une valeur différente pour chaque PPS—deux PPS ne peuvent pas avoir le même code de formation sanitaire. De même, deux membres du personnel du même PPS ne devraient pas avoir la même IDAS. De cette manière, la combinaison de CODEF et de IDAS identifie les prestataires de façon unique. De même, deux clientes observées avec le même prestataire ne devraient pas avoir la même IDCL. La combinaison de CODEFS, IDAS et IDCL devrait identifier chaque cliente de façon unique. Si ces variables n'identifient pas des cas de façon unique, vous ne serez pas en mesure de faire des liaisons entre les modules. Pour cette raison, vérifiez et nettoyez soigneusement ces variables avant de faire des liaisons.

Avec Epi Info, une variable partagée entre deux groupes de données peut lier deux fichiers avec la commande RELATE. Avec SPSS, les variables partagées peuvent lier deux groupes de données avec la commande MATCH FILES avec une sous commande TABLES. Voir les manuels correspondants pour plus d'information sur l'utilisation de ces commandes. 
4. Les scores de connaissance peuvent être calculés de diverses manières. En général, l'objectif est de produire un score pour chaque cliente, qui est facilement interprété, et qui donne une idée du niveau de connaissance qu'elle a sur un sujet donné. Une méthode de calcul qui a été utilisée dans les études d'AS consiste à calculer simplement le pourcentage de questions auxquelles la cliente a répondu correctement.

Par exemple, il y a six questions de connaissance dans l'interview de sortie (question 72 à 77). Dans le modèle le plus simple, la cliente sera créditée d'un score de 0 à 6 , pour indiquer le nombre de questions auxquelles elle a répondu correctement. Cependant, toutes les questions n'ont pas une telle réponse clairement "correcte." Par exemple, la question 76 demande à ce que la cliente énumère les effets secondaires de la pilule qu'elle connaît. Le tableau contient six effets secondaires, mais on ne demandera pas certainement à la cliente de mentionner tous les six. L'analyste doit d'abord décider des limites à fixer-exemple, on peut demander à ce que la cliente mentionne deux ou trois effets secondaires pour être considérée comme connaissant les effets secondaires. De cette manière, si elle mentionne assez d'effets secondaires, elle est considérée comme ayant répondu "correctement" à la question des effets secondaires. Chaque question doit être considérée de cette façon et notée correctement, compte tenu des objectifs et des attentes du programme.
Une fois que chaque question est notée de cette manière, chaque cliente aura un score allant de 0 à 6 . Ceci est utile pour les utilisatrices de la pilule, mais il serait utile aussi de pouvoir comparer la connaissance des clientes de la pilule avec celle des utilisatrices d'autres méthodes.

Mais, si par exemple il y a quatre questions sur les injectables, alors les barèmes sont différents et les résultats ne sont pas comparables. Pour cette raison, les scores de connaissance sont généralement normalisés à un pourcentage. L'utilisatrice de la pilule qui répond correctement à 3 questions sur 6 reçoit un score de 0,50. L'utilisatrice de l'injectable qui répond correctement à 3 questions sur 4 reçoit un score de 0,75 . Ces résultats sont comparables.

Les versions antérieures de l'interview du personnel incluaient des questions de connaissance par méthode. Cela permettait de calculer les scores de connaissance du personnel, ce qui était un indicateur utile de la compétence technique. Malheureusement, les séries de questions extrêmement longues ont suscité des protestations de la part des interviewés, ce qui a entraîné l'annulation de ces questions. Cependant, certaines questions de connaissance restent encore, telles que les méthodes recommandées pour espacer et limiter les naissances, ce qui peut constituer aussi la base des scores de connaissance. 


\section{Listes des échantillons d'indicateurs}

Comme précédemment mentionné, aucun rapport d'AS n'inclura tous les 138 indicateurs énumérés dans le plan d'analyse parce que cela constitue une information trop volumineuse pour être traitée efficacement. De même, beaucoup d'études manipulent les données de manières différentes et conçoivent leurs propres indicateurs qui ne sont pas énumérés ci-dessus. Le résultat est que la liste des indicateurs de chaque pays est unique, ce qui est le produit d'un consensus entre les responsables de programmes et les chercheurs.
En guise de référence, deux de ces listes sont incluses dans les Tableaux 2 et 3, venant du Sénégal et du Botswana. Ce sont les vraies listes qui ont été utilisées pour les analyses primaires et secondaires des données d'AS, et sont utilisées par les responsables de programmes pour évaluer leurs programmes et élaborer des interventions. La liste du Sénégal présente une collection assez standard des indicateurs. Au Botswana, beaucoup de nouveaux indicateurs sur la santé de la reproduction ont été ajoutés, et les instruments de l'Analyse Situationnelle ont subit des changements profonds pour collecter l'information pertinente. 
TABLEAU 2. Indicateurs du Sénégal, 1994

\section{Indicateurs de la capacité de fonctionnement}

\section{Formation}

1. Pourcentage du personnel formé en PF clinique

2. Pourcentage du personnel formé en counseling sur la PF

\section{Service offerts}

3. Pourcentage de PPS offrant le paquet minimum de SMI/PF à la demande

\section{Equipement et provisions}

4. Pourcentage de PPS avec tous les équipements nécessaires pour les services de PF

5. Pourcentage de PPS avec $70 \%$ des équipements nécessaires pour les services de PF

6. Pourcentage de PPS avec $50 \%$ des équipements nécessaires pour la pose/retrait du DIU

\section{Produits et logistique}

7. Pourcentage des PPS avec un inventaire écrit du stock reçu

8. Pourcentage de PPS ayant connu une rupture de stock au cours des six derniers mois

9. Pourcentage de PPS ayant connu une rupture de $\mathrm{COC}$ au cours des six derniers mois

10. Pourcentage de PPS ayant connu une rupture d'injectables au cours des six derniers mois

\section{Supervision et gestion}

11. Pourcentage de PPS ayant reçu une visite de supervision dans les trois mois qui ont précédé l'étude

12. Pourcentage de registres de PF remplis correctement

13. Pourcentage de PPS qui ont envoyé un rapport au cours du dernier mois

\section{Indicateurs de la qualité des soins}

1. Pourcentage de clientes PF capables de fournir la moitié de l'information demandée sur leur méthode

2. ... parmi les utilisatrices de la pilule

3. .... parmi les utilisatrices du DIU

4. ... parmi les utilisatrices de l'injectable

5. .... parmi les utilisatrices du NORPLANT

6. Pourcentage de nouvelles clientes PF qui n'ont pas reçu la méthode choisie (pour des raisons non médicales)

7. Pourcentage de clientes PF avec lesquelles on a discuté au moins d'un autre service

8. Pourcentage de clientes SMI qui ont entendu parler de services de PF de la part d'un prestataire

9. Pourcentage de prestataires qui suivent les normes et les protocoles de prestation des services de SMI/PF:

\section{Comprendre les besoins de la cliente}

a. Le prestataire demande à la cliente ses intentions en matière de procréation

b. Le prestataire demande si la cliente pratique l'allaitement au sein

c. Le prestataire demande aux nouvelles clientes si elles ont des inquiétudes concernant une méthode

d. Le prestataire demande aux anciennes clientes si elles veulent cesser ou changer de méthodes

e. Le prestataire demande aux anciennes clientes si elles ont eu des problèmes avec leur méthode

f. Le prestataire s'occupe des problèmes évoqués par les anciennes clientes

g. Le prestataire discute de MST avec les clientes

h. Moyenne de la compréhension des besoins de la cliente

\section{Choix de la méthode}

a. Le prestataire mentionne au moins une méthode différente de celle acceptée

b. Le prestataire décrit au moins une méthode différente de celle acceptée

c. Le prestataire n'encourage pas ouvertement une méthode particulière

d. Le prestataire impose des barrières sur la moitié ou moins de méthodes offertes au PPS

e. Dans la plupart des cas, le prestataire recommande les méthodes appropriées pour espacer ou limiter les naissances

f. Le prestataire s'assure de la préférence de la cliente pour une méthode

g. Le prestataire envoie les clientes vers un autre PPS si la méthode n'est pas disponible

h. Moyenne du choix des méthodes 
Indicateur de la qualité 9, suite :

\section{Information donnée aux clientes}

a. Le prestataire informe les nouvelles acceptantes sur comment utiliser la méthode

b. Le prestataire informe les nouvelles acceptantes des avantages de la méthode

c. Le prestataire informe les nouvelles acceptantes des inconvénients de la méthode

d. Le prestataire informe les nouvelles acceptantes des effets secondaires de la méthode

e. Le prestataire informe les nouvelles acceptantes de la possibilité de changer de méthodes

f. Le prestataire informe les nouvelles clientes que les préservatifs protègent contre les MSTMIH

g. Le prestataire utilise le matériel d'IEC

h. Le prestataire utilise un échantillon de contraceptif

i. Moyenne de l'information donnée aux clientes

\section{Compétence technique}

a. Le prestataire s'informe des antécédents médicaux

b. Le prestataire demande à la cliente la DDR

c. Le prestataire demande à la cliente les saignements inhabituels

d. Le prestataire demande à la cliente les symptômes de MST

e. Le prestataire prend la TA de la cliente

f. Le prestataire pèse les clientes

g. Le prestataire fait un examen physique

h. Le prestataire fait un examen pelvien

i. Le prestataire suit tous les protocoles de l'examen pelvien

j. Le prestataire suit tous les protocoles pour la pose du DIU

k. Le prestataire suit tous les protocoles pour faire les injectables

I. Le prestataire suit tous les protocoles pour la pose du NORPLANT®

m. Moyenne de la compétence technique

\section{Mécanismes pour assurer la continuité}

a. Le prestataire indique aux clientes la date de leur retour

b. Le prestataire donne aux clientes par écrit ou sous forme non verbale un rappel de la date de retour

c. Le prestataire indique aux clientes le lieu où elles doivent se rendre pour un réapprovisionnement

d. Le prestataire informe les nouvelles acceptantes sur ce qu'elles doivent faire en cas de problèmes

e. Moyenne des mécanismes pour assurer la continuité

\section{Relations interpersonnelles}

a. Le prestataire salue les clientes de manière cordiale

b. Le prestataire a été compris facilement par les clientes

c. Le prestataire donne suffisamment de temps pour la consultation

d. Le prestataire répond aux questions des clientes de manière satisfaisante

e. Les clientes du prestataire pensent que le temps d'attente était raisonnable

f. Les clientes du prestataire pensent que la consultation s'est déroulée dans la plus grande intimité

g. Moyenne des relations interpersonnelles 


\section{Indicateurs de la capacité de fonctionnement}

\section{Formation}

1. Pourcentage du personnel formé en counseling, gestion des MSTNIH, SMI/PF et IEC par type de formation (pré-service, sur le tas, formation continue)

2. Nombre moyen d'années depuis chaque type de formation

\section{Intégration}

3. Pourcentage de formations sanitaires qui offrent des services de SMI/PF et de MSTNIH sur une base quotidienne

4. Pourcentage des prestataires de services de santé qui évaluent les risques de MST chez les clientes SMI/PF

5. Pourcentage des prestataires de services de santé qui font un counseling sur les risques de MST chez les clientes SMI/PF

6. Pourcentage des prestataires de services de santé qui encouragent les méthodes doubles

7. Pourcentage des prestataires de services de santé qui posent des questions aux clientes présentant des MST ou les conseillent sur la PF

8. Pourcentage de clientes recevant des services autres que ceux sollicités à priori

\section{Orientation de nouveaux cadres}

9. Pourcentage de formations sanitaires qui disposent actuellement d'un paquet standard d'orientation

10. Pourcentage de nouveaux cadres qui ont été orientés et qui ont le paquet standard d'orientation

\section{Recherche et évaluation}

11. Pourcentage de projets de recherche planifiés en SR qui ont été réalisés

12. Pourcentage de projets de recherche en SR qui ont été évalués conformément au plan

13. Pourcentage des études de recherche achevées qui ont été diffusées

\section{Equipement et provisions}

14. Pourcentage de formations sanitaires disposant d'un équipement de base fonctionnel standardisé

15. Pourcentage de formations sanitaires disposant d'une provision suffisante (d'une durée de trois mois) de stock non périmé Information, éducation et communication

16. Pourcentage de formations sanitaires disposant d'affiches/boîte à lettre/dépliant sur la SMI, les MSTNIH ou la PF

17. Pourcentage de clientes ayant reçu un matériel d'IEC relatif à l'infrastructure de la SR

18. Pourcentage de formations sanitaires qui disposent de toilettes fonctionnelles pour client(e)s

19. Pourcentage de formations sanitaires qui disposent de source fonctionnelle d'approvisionnement en eau dans la concession

20. Pourcentage de formations sanitaires qui disposent de lieux d'attente protégés

21. Pourcentage de formations sanitaires ayant une salle de counseling/examen séparée

22. Pourcentage de formations sanitaires qui disposent d'une source d'énergie fonctionnelle

\section{Lutte contre l'infection}

23. Pourcentage de formations sanitaires qui disposent d'un équipement pour garder/tenir/décontaminer l'équipement/produits médicaux utilisés (boîte pour outils tranchants, poubelle)

24. Pourcentage de formations sanitaires qui disposent de mécanismes adéquats de traitement des ordures (fosses, flamme, incinération)

\section{Accessibilité}

25. Pourcentage de formations sanitaires observées qui offrent des services de SR après 13 h45

26. Pourcentage de clientes indiquant que le temps d'attente est acceptable

27. Moyenne du temps d'attente

28. Pourcentage de formations sanitaires avec des signes pour faciliter la circulation des clientes

\section{Supervision}

29. Pourcentage de visites planifiées qui ont été honorées

30. Pourcentage de formations sanitaires supervisées qui ont reçu un feed-back 


\section{Gestion}

31. Pourcentage d'ESD qui disposent de guides à jour, de services standard et de manuels de référence

32. Nombre de postes occupés par rapport aux postes établis

33. Pourcentage de formations sanitaires qui ont connu une rupture de stock au cours des 3 derniers mois

34. Pourcentage de formations sanitaires qui disposent d'outils de collecte des données bien remplis (fiches de pointage, registres de stock, formulaires de résumés mensuels, registres, registre des statistiques de l'EBF)

Transport et communication

35. Pourcentage de formations sanitaires/institutions disposant d'un véhicule en bon état

36. Pourcentage de formations sanitaires/institutions disposant d'un système de communication

Qualité des services

Politique

37. Pourcentage de formations sanitaires disposant de documents de politique actuelle

\section{Information donnée à la cliente}

38. Pourcentage de nouvelles clientes informées sur toutes les méthodes appropriées disponibles

39. Pourcentage de nouvelles clientes informées sur la méthode acceptée

\section{Choix des méthodes}

40. Pourcentage de clientes envoyées vers d'autres PPS si la méthode n'est pas disponible

41. Pourcentage de prestataires qui déclarent qu'ils imposent des restrictions sur les méthodes à cause de l'âge, de la parité, du statut matrimonial ou du non consentement de l'époux

\section{Relations interpersonnelles}

42. Pourcentage de clientes qui ont reçu un accueil chaleureux par des salutations et une invite à s'asseoir

43. Pourcentage de clientes qui pensent qu'elles ont été consultées dans l'intimité totale

44. Pourcentage de clientes auxquelles on a permis de poser des questions

45. Pourcentage de clientes satisfaites du temps passé avec le prestataire

\section{Mécanismes pour encourager la continuité}

46. Pourcentage de clientes informées de la date de retour pour un réapprovisionnement/contrôle

47. Pourcentage de clientes qui ont été contactées à travers un suivi après les dates de rendez-vous

\section{Compétence technique}

48. Pourcentage de nouvelles à clientes qui on a fait :

une prise de la tension artérielle

une prise du poids

un examen physique

un examen mammaire

un examen pelvien

un dépistage de MST

une analyse d'urine

I'historique des antécédents médicaux 
49. Pourcentage de prestataires qui peuvent démontrer des compétences dans les procédures cliniques conformément aux directives établies et aux standard des services

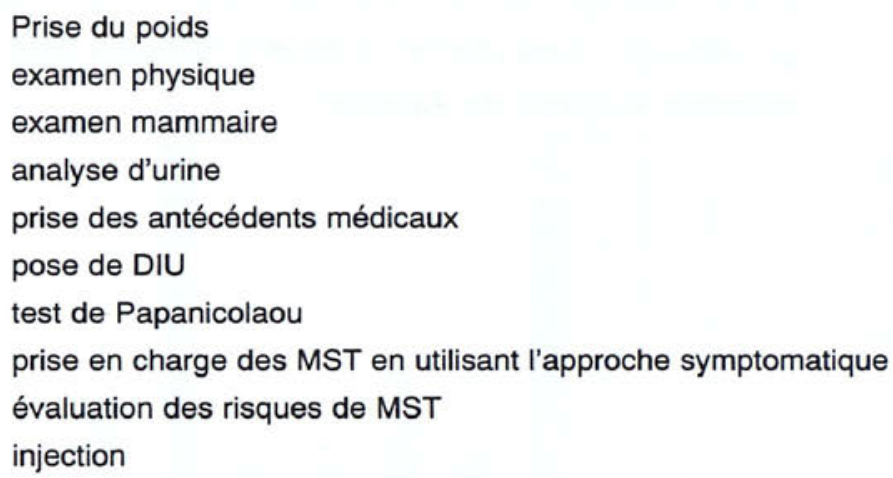

50. Pourcentage de prestataires faisant preuve de compétence dans l'utilisation des aides visuelles pendant le counseling (ex. modèles anatomique, boîtes à image, livrets, affiches)

\section{Impact}

51. Pourcentage de clientes qui connaissent les mesures de prévention des MST/NIH/SIDA

52. Pourcentage de clientes qui connaissent les signes/symptômes des MST/NIH/SIDA

53. Pourcentage de clientes utilisant d'autres méthodes de PF, et qui ont accepté le préservatif pendant cette visite

54. Pourcentage de clientes qui utilisaient des méthodes doubles et qui continuent à les utiliser 


\section{Echantillons de graphiques}

Une fois que les indicateurs ont été sélectionnés et calculés, ils devraient être présentés dans le rapport final de manière aussi efficace que possible. Généralement, les résultats sont présentés sous forme de diagrammes en camembert ou en bâtons, comme approprié, et un rapport type peut contenir jusqu'à soixante de ces graphiques. Ce type de présentation est généralement claire et plus facile à interpréter que les tableaux avec des chiffres.
Les douze graphiques suivants donnent des exemples de quelques-uns des résultats d'AS les plus courants et la manière dont ils peuvent être présentés de manière efficace sous forme de graphiques. Les données proviennent d'une étude récente réalisée en Afrique. 

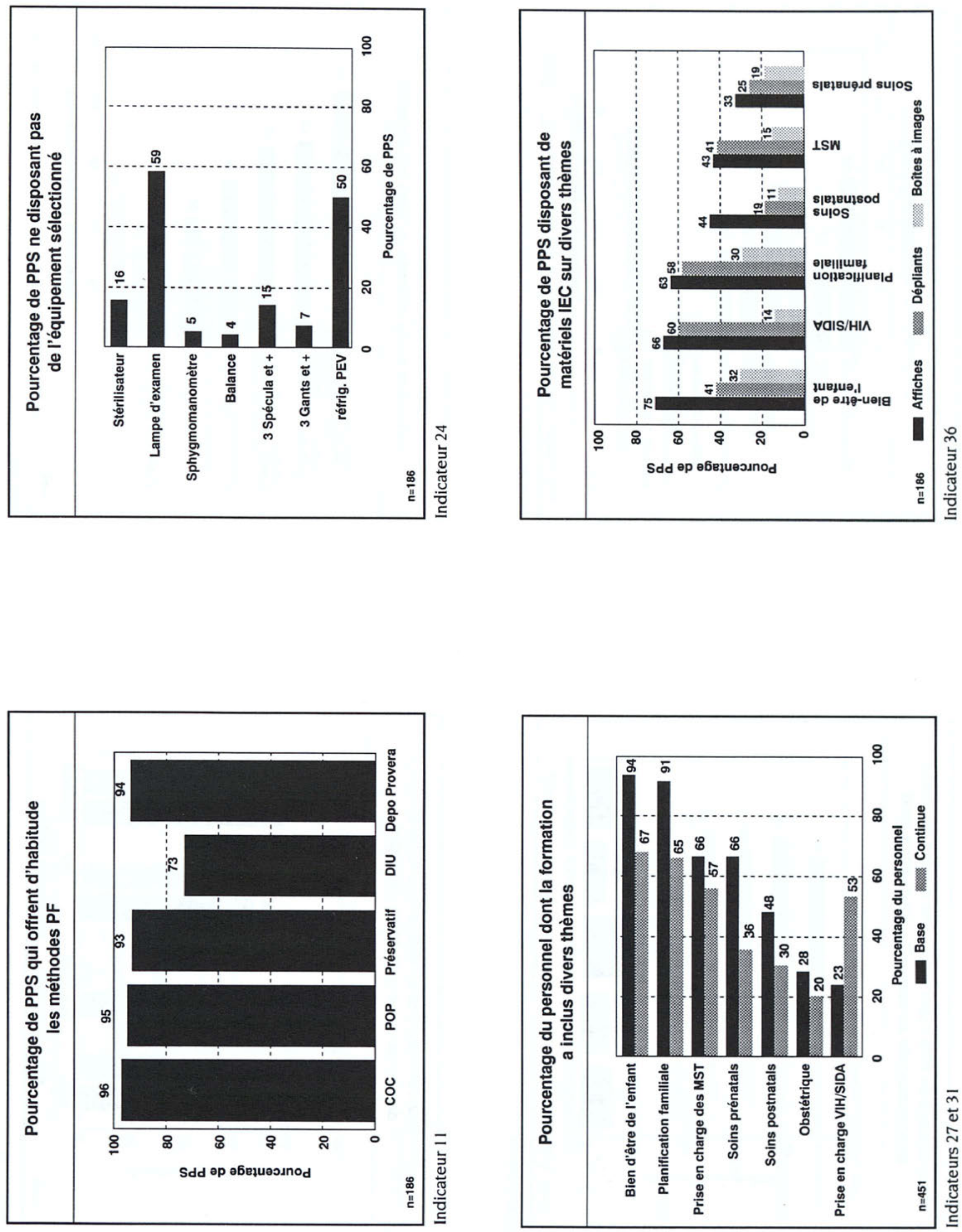

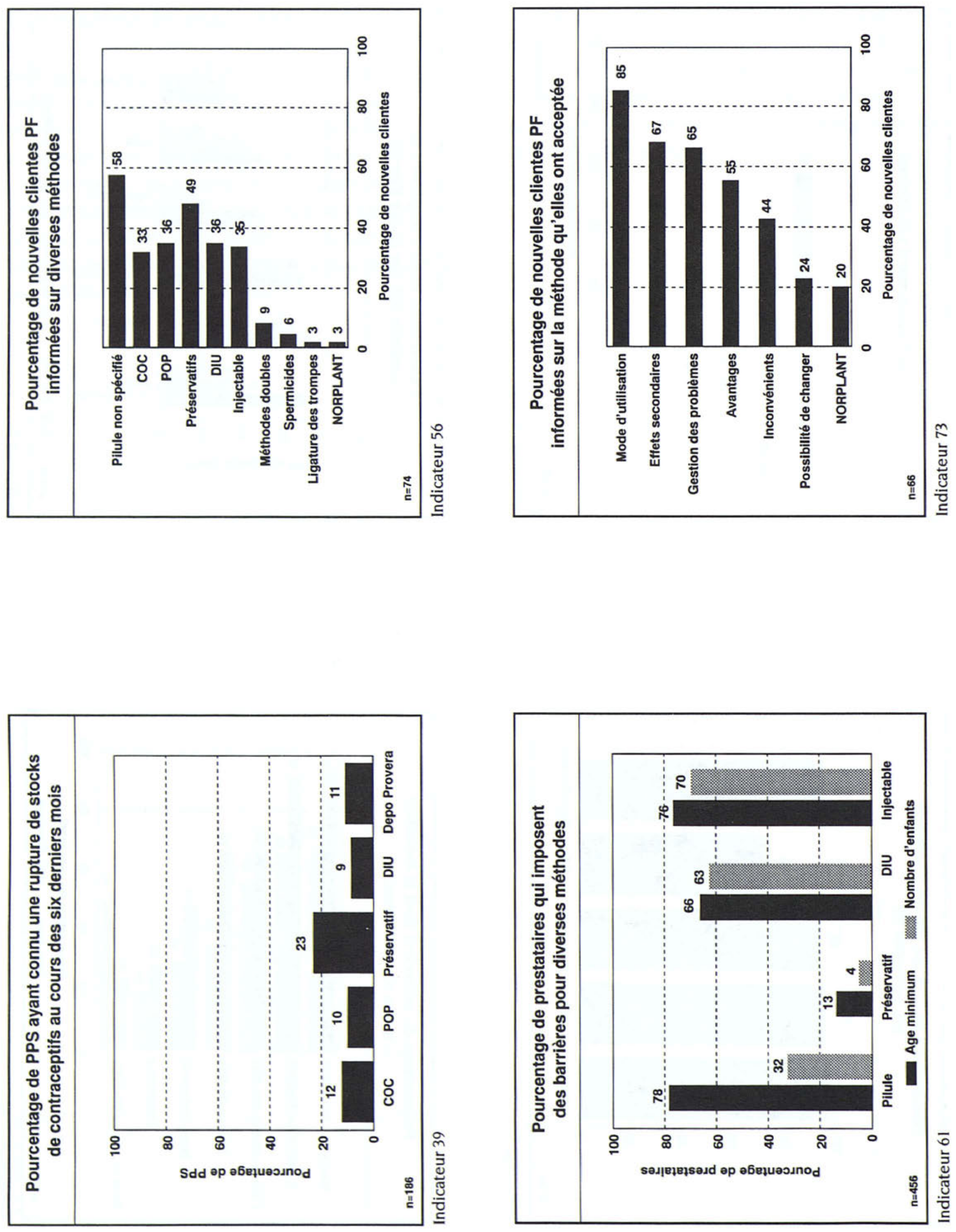

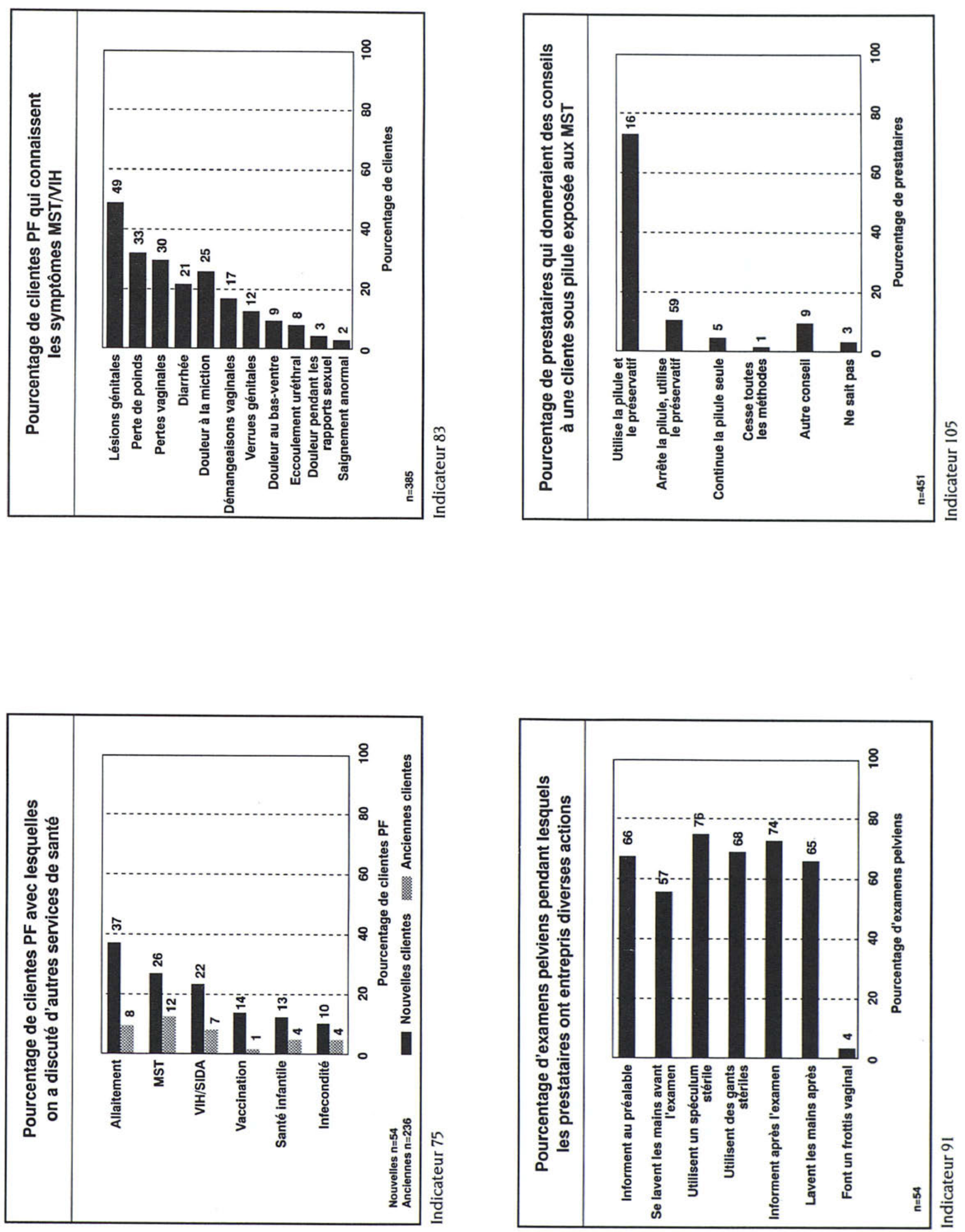






\title{
(7) Population Council
}

\author{
Projet de Recherche Opérationnelle et \\ Afrt d'Assistance Technique en Afrique
}

